\title{
Strategies to improve the implementation of healthy eating, physical activity and obesity prevention policies, practices or programmes within childcare services (Review)
}

Wolfenden L, Barnes C, Jones J, Finch M, Wyse RJ, Kingsland M, Tzelepis F, Grady A, Hodder RK, Booth D, Yoong SL

Wolfenden L, Barnes C, Jones J, Finch M, Wyse RJ, Kingsland M, Tzelepis F, Grady A, Hodder RK, Booth D, Yoong SL.

Strategies to improve the implementation of healthy eating, physical activity and obesity prevention policies, practices or programmes within childcare services.

Cochrane Database of Systematic Reviews 2020, Issue 2. Art. No.: CD011779.

DOI: 10.1002/14651858.CD011779.pub3.

www.cochranelibrary.com

Strategies to improve the implementation of healthy eating, physical activity and obesity prevention policies, practices or programmes within childcare services (Review) 
TABLE OF CONTENTS

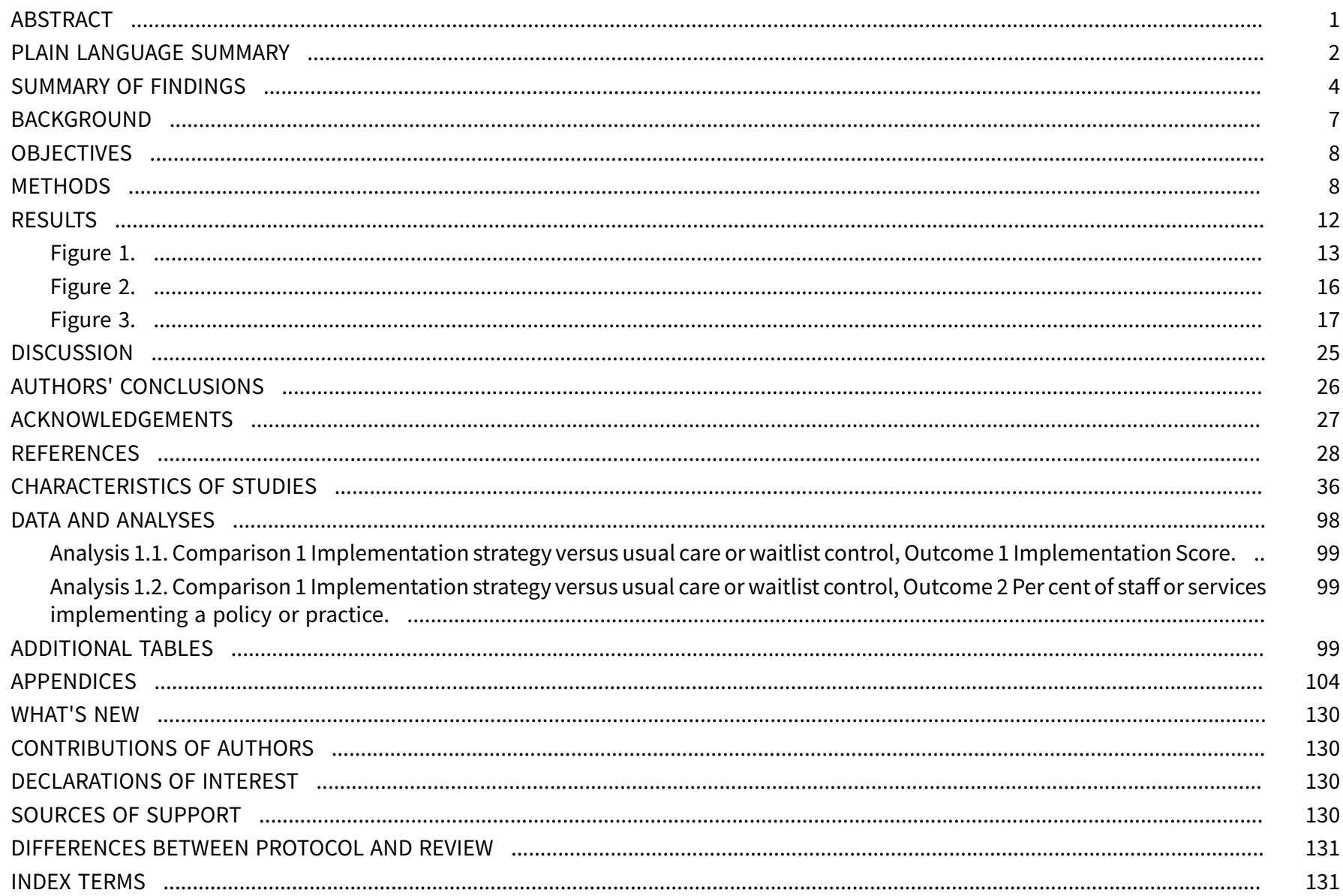


[Intervention Review]

\section{Strategies to improve the implementation of healthy eating, physical activity and obesity prevention policies, practices or programmes within childcare services}

Luke Wolfenden 1,2,3, Courtney Barnes ${ }^{3}$, Jannah Jones ${ }^{1,2,3}$, Meghan Finch1,2,3, Rebecca J Wyse1,2,3, Melanie Kingsland ${ }^{1}$, Flora Tzelepis ${ }^{1}$, Alice Grady ${ }^{1}$, Rebecca K Hodder ${ }^{3}$, Debbie Booth ${ }^{4}$, Sze Lin Yoong1,2,3

1School of Medicine and Public Health, University of Newcastle, Callaghan, Australia. ${ }^{2}$ Hunter Medical Research Institute, New Lambton, Australia. ${ }^{3}$ Hunter New England Population Health, Hunter New England Local Health District, Wallsend, Australia. ${ }^{4}$ Auchmuty Library, University of Newcastle, Callaghan, Australia

Contact: Luke Wolfenden, School of Medicine and Public Health, University of Newcastle, Callaghan, NSW, 2308, Australia.

luke.wolfenden@health.nsw.gov.au.

Editorial group: Cochrane Public Health Group.

Publication status and date: Edited (conclusions changed), published in Issue 2, 2020.

Citation: Wolfenden L, Barnes C, Jones J, Finch M, Wyse RJ, Kingsland M, Tzelepis F, Grady A, Hodder RK, Booth D, Yoong SL. Strategies to improve the implementation of healthy eating, physical activity and obesity prevention policies, practices or programmes within childcare services. Cochrane Database of Systematic Reviews 2020, Issue 2. Art. No.: CD011779. DOI: 10.1002/14651858.CD011779.pub3.

Copyright @ 2020 The Cochrane Collaboration. Published by John Wiley \& Sons, Ltd.

\section{A B S T R A C T}

\section{Background}

Despite the existence of effective interventions and best-practice guideline recommendations for childcare services to implement evidence-based policies, practices and programmes to promote child healthy eating, physical activity and prevent unhealthy weight gain, many services fail to do so.

\section{Objectives}

The primary aim of the review was to examine the effectiveness of strategies aimed at improving the implementation of policies, practices or programmes by childcare services that promote child healthy eating, physical activity and/or obesity prevention.

The secondary aims of the review were to:

1. Examine the cost or cost-effectiveness of such strategies;

2. Examine any adverse effects of such strategies on childcare services, service staff or children;

3. Examine the effect of such strategies on child diet, physical activity or weight status.

4. Describe the acceptability, adoption, penetration, sustainability and appropriateness of such implementation strategies.

\section{Search methods}

We searched the following electronic databases on February 22 2019: Cochrane Central Register of Controlled trials (CENTRAL), MEDLINE, MEDLINE In Process, Embase, PsycINFO, ERIC, CINAHL and SCOPUS for relevant studies. We searched reference lists of included studies, handsearched two international implementation science journals, the World Health Organization International Clinical Trials Registry Platform (www.who.int/ictrp/) and ClinicalTrials.gov (www.clinicaltrials.gov).

\section{Selection criteria}

We included any study (randomised or nonrandomised) with a parallel control group that compared any strategy to improve the implementation of a healthy eating, physical activity or obesity prevention policy, practice or programme by staff of centre-based childcare

Strategies to improve the implementation of healthy eating, physical activity and obesity prevention policies, practices or programmes 
services to no intervention, 'usual' practice or an alternative strategy. Centre-based childcare services included preschools, nurseries, long daycare services and kindergartens catering for children prior to compulsory schooling (typically up to the age of five to six years).

\section{Data collection and analysis}

Two review authors independently screened study titles and abstracts, extracted study data and assessed risk of bias; we resolved discrepancies via consensus. We performed meta-analysis using a random-effects model where studies with suitable data and homogeneity were identified; otherwise, findings were described narratively.

\section{Main results}

Twenty-one studies, including 16 randomised and five nonrandomised, were included in the review. The studies sought to improve the implementation of policies, practices or programmes targeting healthy eating (six studies), physical activity (three studies) or both healthy eating and physical activity (12 studies). Studies were conducted in the United States $(n=12)$, Australia $(n=8)$ and Ireland $(n=1)$. Collectively, the 21 studies included a total of 1945 childcare services examining a range of implementation strategies including educational materials, educational meetings, audit and feedback, opinion leaders, small incentives or grants, educational outreach visits or academic detailing, reminders and tailored interventions. Most studies $(n=19)$ examined implementation strategies versus usual practice or minimal support control, and two compared alternative implementation strategies. For implementation outcomes, six studies (one RCT) were judged to be at high risk of bias overall.

The review findings suggest that implementation strategies probably improve the implementation of policies, practices or programmes that promote child healthy eating, physical activity and/or obesity prevention in childcare services. Of the 19 studies that compared a strategy to usual practice or minimal support control, 11 studies (nine RCTs) used score-based measures of implementation (e.g. childcare service nutrition environment score). Nine of these studies were included in pooled analysis, which found an improvement in implementation outcomes (SMD 0.49; $95 \% \mathrm{Cl} 0.19$ to 0.79; participants $=495$; moderate-certainty evidence). Ten studies (seven RCTs) used dichotomous measures of implementation (e.g. proportion of childcare services implementing a policy or specific practice), with seven of these included in pooled analysis (OR 1.83; $95 \% \mathrm{Cl} 0.81$ to 4.11 ; participants = 391; low-certainty evidence).

Findings suggest that such interventions probably lead to little or no difference in child physical activity (four RCTs; moderate-certainty evidence) or weight status (three RCTs; moderate-certainty evidence), and may lead to little or no difference in child diet (two RCTs; lowcertainty evidence). None of the studies reported the cost or cost-effectiveness of the intervention. Three studies assessed the adverse effects of the intervention on childcare service staff, children and parents, with all studies suggesting they have little to no difference in adverse effects (e.g. child injury) between groups (three RCTs; low-certainty evidence). Inconsistent quality of the evidence was identified across review outcomes and study designs, ranging from very low to moderate.

The primary limitation of the review was the lack of conventional terminology in implementation science, which may have resulted in potentially relevant studies failing to be identified based on the search terms used.

\section{Authors' conclusions}

Current research suggests that implementation strategies probably improve the implementation of policies, practices or programmes by childcare services, and may have little or no effect on measures of adverse effects. However such strategies appear to have little to no impact on measures of child diet, physical activity or weight status.

\section{PLAIN LANGUAGE SUMMARY}

Improving the implementation of healthy eating, physical activity and obesity prevention policies, practices or programmes in childcare services

\section{The review question}

This review aimed to look at the effects of strategies to improve the implementation (or correct undertaking) of policies, practices or programmes by childcare services that promote children's healthy eating, physical activity and/or obesity prevention. We wanted to determine the cost or cost-effectiveness of providing implementation support, whether support strategies were associated with any adverse effects, and whether there was an impact on child nutrition, physical activity or weight status. We also looked at the implementation strategy acceptability, adoption, penetration, sustainability and appropriateness.

\section{Background}

A number of childcare service-based interventions have been found to be effective in improving child diet, increasing child physical activity and preventing excessive weight gain. Despite the existence of such evidence and best-practice guideline recommendations for childcare services to implement these policies, practices or programmes, many childcare services fail to do so. Without proper implementation, children will not benefit from these child health-directed policies, practices or programmes.

\section{Study characteristics}

We identified 21 studies, 19 of which examined implementation strategies versus usual practice or minimal support control, and two that compared different types of implementation strategies. The studies sought to improve the implementation of policies, practices or

Strategies to improve the implementation of healthy eating, physical activity and obesity prevention policies, practices or programmes 
programmes targeting healthy eating (six studies), physical activity (three studies) or both healthy eating and physical activity (twelve studies). Collectively, the 21 included studies included a total of 1945 childcare services and examined a range of implementation strategies including educational materials, educational meetings, audit and feedback, opinion leaders, small incentives or grants, educational outreach visits or academic detailing, reminders and tailored interventions. The strategies tested were only a small number of those that could be applied to improve implementation in this setting.

\section{Search date}

The evidence is current to February 2019.

\section{Key results}

Findings suggest that implementation support strategies can improve the implementation of physical activity policies, programmes or practices by childcare services or their staff (moderate-certainty evidence), and do not appear to increase the risk of child injury (lowcertainty evidence). However, such approaches do not appear to have an impact on the diet, physical activity or weight status of children (low to moderate-certainty evidence). None of the included studies reported information regarding implementation strategy costs or measures of cost-effectiveness. The lack of consistent terminology in this area of research may have meant some relevant studies were not picked up in our search. 


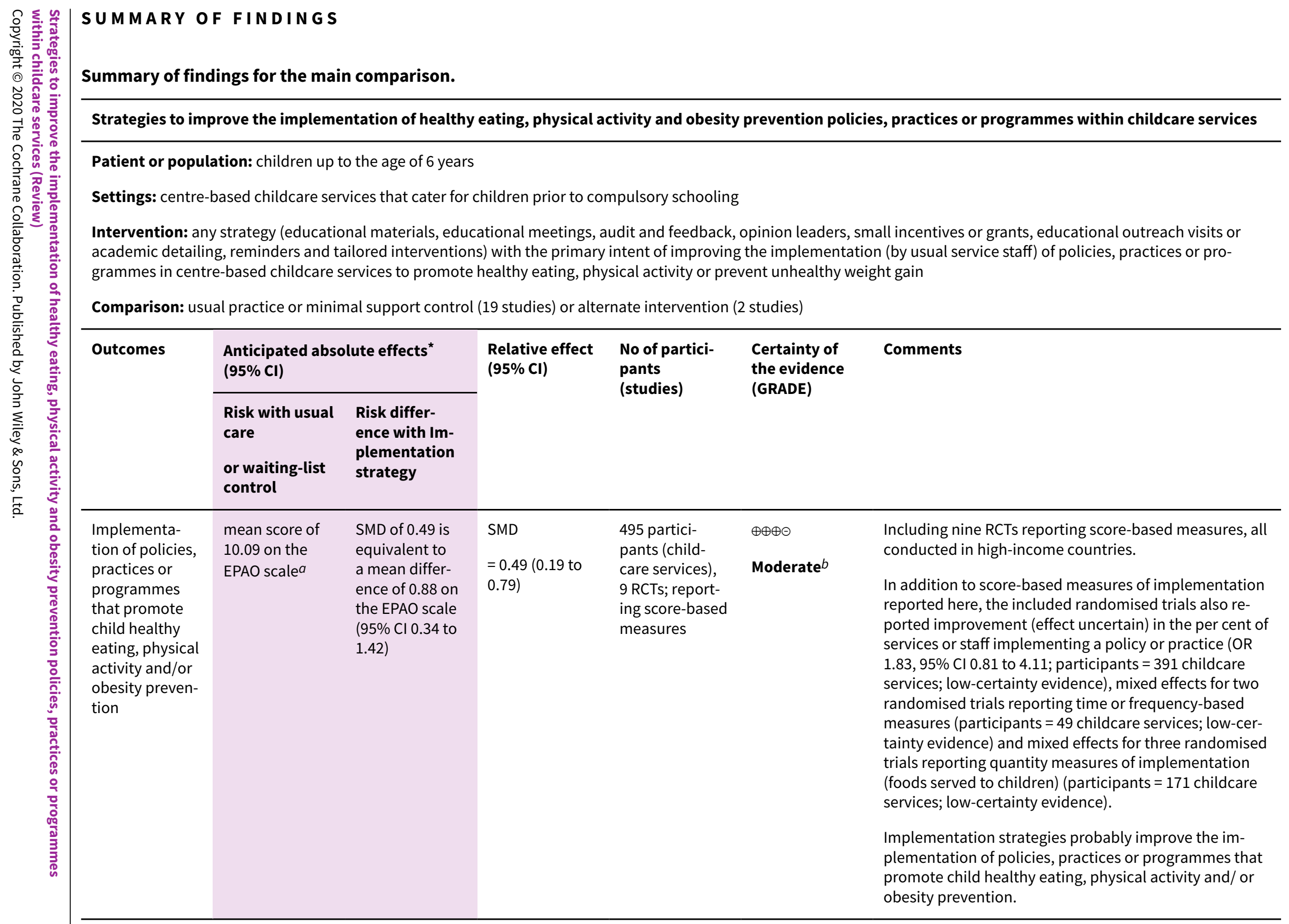


Cost or costeffectiveness of strategies to improve the implementa-

tion of policies, practices or

programmes in childcare ser-

vices

Adverse con-

sequences of

strategies to

improve the

implementa-

tion of policies,

practices or

programmes in

childcare ser-

vices

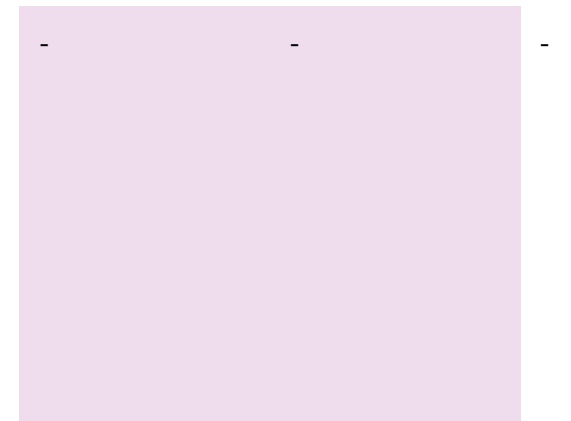

pants (child-

care services), 2

RCTs; reporting

continuous out-

comes (rates of

child injury)

$\oplus \oplus \odot \Theta$

Low
No studies were found that looked at the cost or costeffectiveness of strategies to improve the implementation of policies, practices or programmes in childcare services.

countries. Across the two RCTs that reported continuous

measures of adverse effects (rates of child injury) there

were no clear differences reported between groups in rates of child injuries.

Similarly, there was no difference between groups in a single trial reporting dichotomous outcomes (reported complaints received by services) (participants $=45$ childcare services; very low-certainty evidence).

Strategies to improve the implementation of policies, practices or programmes that promote child healthy eating, physical activity and/or obesity prevention may have little to no impact on measures of adverse consequences.

\begin{tabular}{|c|c|c|c|}
\hline $\begin{array}{l}\text { Measures of } \\
\text { child diete }\end{array}$ & - & - & $\begin{array}{l}134 \text { partici- } \\
\text { pants from } 182 \\
\text { childcare ser- } \\
\text { vices), } 2 \text { RCTs, } \\
\text { reporting con- } \\
\text { tinuous (serve- } \\
\text { based mea- } \\
\text { sures) of dietary } \\
\text { intake }\end{array}$ \\
\hline $\begin{array}{l}\text { Measures of } \\
\text { child physical } \\
\text { activity } f\end{array}$ & - & - & $\begin{array}{l}53 \text { childcare } \\
\text { services } \\
\text { ( } 2 \text { RCTs) } \\
\text { reporting di- } \\
\text { chotomous ob- } \\
\text { servational out- } \\
\text { comesg (no. }\end{array}$ \\
\hline
\end{tabular}

$\oplus \oplus \ominus \ominus$

Lowc,d

Including two RCTs, both conducted in high-income

countries. Findings regarding beneficial effects for this outcome were mixed across the two randomised trials.

Strategies to improve the implementation of policies, practices or programmes that promote child healthy eating, physical activity and/or obesity prevention may lead to little or no difference in child diet intake. $\oplus \oplus \oplus \ominus$

Moderate $c$
Including two RCTs, both conducted in high-income countries. The two trials reporting dichotomous observation-based measures of physical activity reported little to no improvement in student physical activity.

Additionally, two trials using continuous and objective measures of child physical activity (e.g. pedometers) (participants $=420$ children from 46 services; high-cer- 
children not re- $\quad$ tainty evidence) reported little to no improvement in ported)

student physical activity.

Strategies to improve the implementation of policies, practices or programmes that promote child healthy eating, physical activity and/or obesity prevention probably lead to little or no difference in child physical activity.

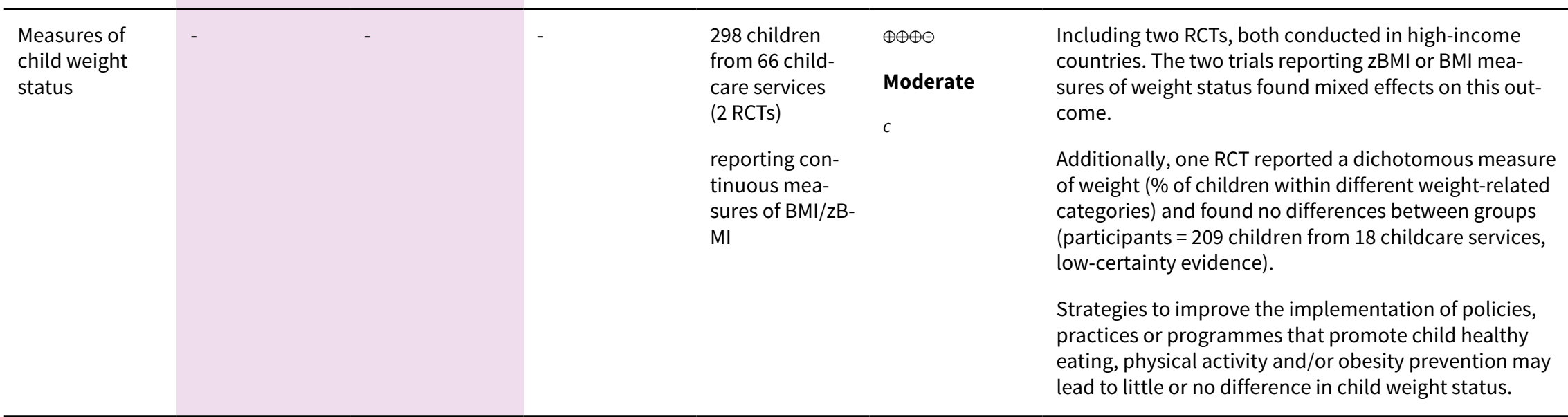

\section{GRADE Working Group grades of evidence}

High quality: We are very confident that the true effect lies close to that of the estimate of the effect

Moderate quality: We are moderately confident in the effect estimate: the true effect is likely to be close to the estimate of the effect, but there is a possibility that it is substantially different

Low quality: Our confidence in the effect estimate is limited: the true effect may be substantially different from the estimate of the effect

Very low quality: We have very little confidence in the effect estimate: the true effect is likely to be substantially different from the estimate of effect

$a$ Risk with usual care or waiting list control calculated as the mean Environment and Policy Assessment and Observation (EPAO) score for the control group as reported in Ward 2017

bDowngraded one level for risk of bias: studies assessed as high and unclear risk of bias for the majority of domains.

cDowngraded one level for inconsistency: narrative synthesis indicated a high level of inconsistency in results across studies and outcomes measured within studies.

$d$ Downgraded one level for imprecision: total sample size $<400$

e Measures of child diet: included child consumption of food groups (e.g. fruit and vegetables) measured via weighed food records and researcher observations

fMeasures of child physical activity: included frequency and duration of child physical activity (e.g. step count), measured via pedometers, accelerometers and researcher observations

gDichotomous observational outcomes: included type and intensity of child physical activity (e.g. very active, walking, sedentary), measured via researcher observations 


\section{B A C K G R O U N D}

\section{Description of the condition}

Internationally, the prevalence of being overweight or obese has increased across every region of the world in recent decades (Finucane 2011). Currently, over 1.9 billion adults and 340 million children are overweight or obese (World Health Organization 2018). While obesity rates in high-income countries remain higher, prevalence rates in low- and middle-income countries are accelerating (Swinburn 2011). In Africa, for example, the prevalence of being overweight among children under five years is expected to increase from 4\% in 1990 to $11 \%$ by 2025 (Black 2013). Excessive weight gain increases the risk of a variety of chronic health conditions. Between the years 2010 and 2030, up to 8.5 million cases of diabetes, 7.3 million cases of heart disease and stroke, and 669,000 cases of cancer attributable to obesity have been projected in the USA and UK alone (Wang 2011). In Australia, between the years 2011 and 2050, 1.75 million lives and over 10 million premature years of life will be lost due to excessive weight gain (Gray 2009).

\section{Description of the intervention}

Physical inactivity and poor diet are key drivers of excessive weight gain. As excessive weight gain in childhood tracks into adulthood, interventions targeting children's diet and physical activity have been recommended to mitigate the adverse health effects of obesity on the population (World Health Organization 2012). A recently published World Health Organization report into population-based approaches to childhood obesity prevention identified centre-based childcare services (including preschools, long daycare services and kindergartens that provide educational and developmental activities for children prior to formal compulsory schooling) as an important setting for public health action to reduce the risk of unhealthy weight gain in childhood. Such settings provide an opportunity to access large numbers of children for prolonged periods of time (World Health Organization 2012). Further, randomised and nonrandomised studies have identified a number of interventions, delivered in childcare services, which have increased child physical activity and fundamental movement skill proficiency, improved child diet quality and prevented excessive weight gain (Brown 2019; Finch 2016; Stacey 2017). As such, regulations and best practice guidelines for the childcare sector recommend implementation of a number of healthy eating and physical activity policies and practices, such as restricting sedentary screen time opportunities; ensuring meals provided by childcare services or foods packed by parents for consumption in care are consistent with dietary guidelines; and the provision of programmes to promote physical activity and fundamental movement skill development (Commonwealth of Australia; McWilliams 2009; Tremblay 2012).

Despite the existence of evidence-based best-practice guidelines for childcare services, implementation of obesity prevention policies and practices that are consistent with such guidelines is poor (McWilliams 2009; Wolfenden 2015a). In the USA, a menu audit in 83 childcare centres determined that the menus did not provide the recommended amount of carbohydrates, dietary fibre and iron, whilst providing excessive amounts of sodium (Frampton 2014). Childcare service adherence to dietary guidelines in other countries has also been reported to be poor (Grady 2018; Yoong 2014). Similarly, adherence to best-practice recommendations for physical activity is also suboptimal. For example, only $14 \%$ of USA childcare services provided 120 minutes of active play per day, $57 \%$ to $60 \%$ did not have a written physical activity policy (McWilliams 2009; Sisson 2012), and in $18 \%$ of childcare services, children were seated for more than 30 minutes at a time (McWilliams 2009). In Australia, it has been reported that just $58 \%$ of centre-based childcare services had written healthy eating and physical activity policies (Wolfenden 2015a), and 60\% of child lunch boxes contained more than one serving of high-fat, salt or sugar foods or drinks (Kelly 2010). Similarly in New Zealand, it has been reported that only $35 \%$ of childcare services had a written physical activity policy (Gerritsen 2016).

Without adequate implementation across the population of childcare services, the potential public health benefits of initiatives to improve healthy eating or physical activity, or prevent obesity, will not be fully realised. 'Implementation' is described as the use of strategies to adopt and integrate evidence-based health interventions and to change practice patterns within specific settings (Glasgow 2012). Implementation research, specifically, is the study of strategies designed to integrate health policies, practices or programmes within specific settings (for example, primary care, community centres or childcare services) (Schillinger 2010). The National Institute of Health recognises implementation research as a fundamental component of the third stage of the research translation process ('T3') and that it is a necessary prerequisite for research to yield public health improvements (Glasgow 2012). While staff of centre-based childcare services are responsible for providing educational experiences and an environment supportive of healthy growth and development, including initiatives designed to reduce the risk of excessive weight gain, it may be the childcare services themselves, government or other agencies (such as for licensing and accreditation requirements) that undertake strategies aimed at enhancing the implementation of such initiatives.

There are a range of potential strategies that can improve the likelihood of implementation of healthy eating, physical activity and obesity prevention policies, practices or programmes in childcare services. The Cochrane Effective Practice and Organisation of Care (EPOC) taxonomy is a framework for characterising educational, behavioural, financial, regulatory and organisational interventions (EPOC 2015); it includes three categories with 22 subcategories within the topic of 'implementation strategies'. Examples of such subcategories include continuous quality improvement, educational materials, performance monitoring, local consensus processes, and educational outreach visits (EPOC 2015).

\section{How the intervention might work}

The determinants of policy and practice implementation are complex and the mechanisms by which support strategies facilitate implementation are not well understood. Implementation frameworks have identified a large number of factors operating at multiple macro and micro levels that can influence the success of implementation (Damschroder 2009). However, few studies have been conducted in the childcare setting to identify key determinants of implementation in this setting. A study by Wolfenden and colleagues of over 200 childcare services in Australia examined associations between the existence of healthy eating and physical activity policies and practices and 13 factors suggested by Damschroder's Consolidated Framework for Implementation 
Research to impede or promote implementation (Wolfenden 2015a). The study reported that implementation policy and practice implementation was more likely when service managers, management committee and parents were supportive, and where external resources to support implementation were accessible. Applied implementation frameworks, such as the Theoretical Domains Framework (Michie 2008), suggest that strategies to facilitate implementation may be most likely to be effective with a thorough understanding of the local implementation context and barriers, and when theoretical frameworks are applied to select implementation support strategies to address key determinants of implementation. For example, knowledge barriers to implementation may be best overcome with education meetings or materials, while activity reminders, such as decision support systems, may be particularly important in instances where staff forgetfulness is identified as a local implementation barrier.

\section{Why it is important to do this review}

A number of large systematic reviews have been undertaken to assess the effectiveness of such implementation strategies in improving the professional practice of clinicians. For example, Ivers and colleagues reviewed the effectiveness of audit and feedback on the behaviour of health professionals and the health of their patients, and found such strategies generally resulted in small but important improvements in professional practice (Ivers 2012). Other reviews have examined the impact of printed education materials (Giguère 2012), reminders (Arditi 2012), education meetings and workshops (Forsetlund 2009; O'Brien 2007), incentives (Scott 2011), and other strategies on improving professional practice and implementation of evidencebased interventions by clinicians. Public health implementation research in nonclinical community settings, while still sparse (Buller 2010), is emerging (Wolfenden 2016; Wolfenden 2019). Systematic reviews of the effects of strategies to implement interventions targeting risks of chronic disease in settings such as workplaces (Wolfenden 2018), sporting clubs (McFadyen 2018) and schools (Wolfenden 2017) report an acceleration in the number of published implementation studies over recent years. Such an increase is consistent with an increase in implementation research occurring more broadly in the field (Wilson 2017).

Similarly, our 2016 Cochrane systematic review examining the effects of implementation strategies in childcare identified just 10 studies, providing low-certainty evidence. Since the conduct of this review, we are aware of a number of studies that are currently underway or have been completed (Finch 2019; Mazzucca 2017; Stookey 2017; Ward 2017). Given the current uncertainty of the existing evidence base, the importance of childcare as a setting for health promotion, and the need among policy makers and practitioners for evidence-based implementation strategies for this setting, an update of the review is timely.

\section{O B J E C T IVES}

The primary aim of the review was to examine the effectiveness of strategies aimed at improving the implementation of policies, practices or programmes by childcare services that promote child healthy eating, physical activity and/or obesity prevention.

The secondary aims of the review were to:

1. Examine the cost or cost-effectiveness of such strategies;
2. Examine any adverse effects of such strategies on childcare services, service staff or children;

3. Examine the effect of such strategies on child diet, physical activity or weight status;

4. Describe the acceptability, adoption, penetration, sustainability and appropriateness of such implementation strategies.

\section{METHODS}

\section{Criteria for considering studies for this review \\ Types of studies}

Any study (randomised, including cluster-randomised, or nonrandomised) with a parallel control group that compared:

1. a strategy to improve the implementation of any healthy eating, physical activity or obesity prevention policy, practice or programme in centre-based childcare services compared with no intervention or 'usual' practice;

2. two or more alternative strategies to improve the implementation of any healthy eating, physical activity or obesity prevention policy, practice or programme in centrebased childcare services.

There was no restriction on the length of the study follow-up period, language of publication or country of origin.

\section{Types of participants}

Centre-based childcare services (and staff thereof) such as preschools, nurseries, long daycare services and kindergartens that cater for children prior to compulsory schooling (typically up to the age of five to six years). We excluded studies of childcare services provided in the home and specialised daycare services.

\section{Types of interventions}

Any strategy with the primary intent of improving the implementation of policies, practices or programmes in centrebased childcare services to promote healthy eating, physical activity or prevent unhealthy weight gain was eligible. To be eligible, strategies must have sought to improve the implementation of policies, practices or programmes by usual childcare service staff. Strategies could have included quality improvement initiatives, education and training, performance feedback, prompts and reminders, implementation resources, financial incentives, penalties, communication and social marketing strategies, professional networking, the use of opinion leaders, or implementation consensus processes. Interventions may have been singular or multi-component.

\section{Types of outcome measures}

\section{Primary outcomes}

We included any measure of either the completeness or the quality of the implementation of childcare service policies, practices or programmes (for example, the percentage of childcare services implementing a food service consistent with dietary guidelines or the mean number of physical activity practices implemented). To assess the review outcomes, data may have been collected from a variety of sources including educators, managers, cooks or other staff of centre-based childcare services; or administrators, officials or other health, education, government 
or non-government personnel responsible for encouraging or enforcing the implementation of health-promoting initiatives in childcare services. Such data may have been obtained from audits of service records, questionnaires or surveys of staff, service managers, other personnel or parents; direct observation or recordings; examination of routine information collected from government departments (such as compliance with food standards or breaches of childcare service regulations) or other sources. Additionally, children, parents or childcare service staff may have provided information regarding child diet, physical activity or child weight status.

\section{Secondary outcomes}

1. Estimates of absolute costs or any assessment of the costeffectiveness of strategies to improve the implementation of policies, practices or programmes in childcare services.

2. Any reported adverse consequences of a strategy to improve the implementation of policies, practices or programmes in childcare services. This could include impacts on child health (for example, an increase in child injury following the implementation of physical activity-promoting practices) or child development, service operation or staff attitudes (for example, impacts on staff motivation or cohesion) or the displacement of other key programmes, curricula or practices.

3. Any measure of child diet, physical activity (including sedentary behaviours) or weight status. Such measures could be derived from any data source including direct observation, questionnaire, or anthropometric assessments. We excluded studies focusing on malnutrition/malnourishment.

4. Any measure of acceptability, adoption, penetration, sustainability and appropriateness of the implementation support strategy (Proctor 2011). Such measures are typically included in the experimental arm of the study only, that is, those exposed to an implementation strategy or intervention. As such, we reported within-group findings of these measures for completeness, to improve external validity and enable end-user assessments of potential utility of strategies to implement an evidence-based intervention. The definition of these outcomes were adapted, based on those defined by Proctor, to be as follows:

- Acceptability: The perception among implementation stakeholders that a given policy, practice or programme or strategies to support its implementation is agreeable, palatable or satisfactory (Proctor 2011). Measures assessed at the individual or organisational level were included such a surveys of staff or managers of childcare services regarding their experience of features of the intervention or implementation strategy.

- Penetration: The integration of a policy, practice or programme or strategies to support its implementation within a service setting or its sub settings. Penetration could be measured from the perspective of the provider, service or child individual. We included any measure of penetration at the individual or organisational level (Proctor 2011). For example, the proportion of eligible childcare services that received implementation support strategies, or the proportion of childrens' exposure to targeted intervention.

- Adoption: The intention, including the initial decision, or action to try and implement a policy, practice or programme (Proctor 2011). Adoption could be measured from the perspective of the provider or service. These could include decisions by managers of childcare services to take up a potentially effective intervention, or decisions by individual childcare staff to deliver potential intervention components.

- Sustainability: The extent to which a policy, practice or programme is maintained (Proctor 2011). Measures of sustainability must require successful implementation in part or in full, of an intervention, programme or service that is then sustained for a period of at least six months. This could include the proportion of childcare services maintaining implementation of targeted policy practices or programmes 12 months following the provision of implementation support.

- Appropriateness: The perceived fit, relevance or compatibility of policy, practice or programme or strategies to support its implementation for a given setting, provider or consumer, and/or the perceived fit of the intervention to address a particular problem (Proctor 2011). Measures of appropriateness assessed at the individual or organisational level will be included, such as surveys of staff or managers of childcare services regarding their perception of the congruence of the implementation of a targeted policy, practice or programme with their skill set or work expectations.

\section{Search methods for identification of studies}

We conducted searches for peer-reviewed articles in electronic databases. We also undertook handsearching within relevant journals and reference lists of included studies.

\section{Electronic searches}

For this update, we conducted searches in the following electronic databases on February 22, 2019: the Cochrane Central Register of Controlled trials (CENTRAL) (2019) via Cochrane Library; MEDLINE (1946 to February 22, 2019), MEDLINE In Process (February 22, 2019), PsycINFO (1950 to February 22, 2019) and Embase (1947 to February 22, 2019) via OVID; ERIC (February 22, 2019) via Proquest; CINAHL (February 22, 2019) via EBSCO; and SCOPUS (February 22, 2019) via SCOPUS.

We adapted the MEDLINE search strategy for the other databases and we included filters used in other systematic reviews for population (childcare services) (Zoritch 2000), physical activity (Dobbins 2013), healthy eating (Jaime 2009), and obesity (Waters 2011). A search filter for intervention type (implementation interventions) was based on previous reviews (Rabin 2010), and a glossary of terms in implementation and dissemination research (Rabin 2008). See Appendix 1 for the detailed search strategy.

Small amendments to the original search strategy were made to improve the sensitivity of the search, which was performed by an experienced librarian (DB). After removal of duplicates, citations were exported and managed in Covidence.

\section{Searching other resources}

We searched the reference lists of all included studies for citation of other potentially relevant studies. We conducted handsearches of all publications for the past three years in the journal Implementation Science and the Journal of Translational Behavioural Medicine, as they are the leading implementation journals in the field. Furthermore, we conducted searches 
of the World Health Organization International Clinical Trials Registry Platform (www.who.int/ictrp/) and ClinicalTrials.gov (www.clinicaltrials.gov). We included studies identified in such searches, which have not yet been published, in the 'Characteristics of ongoing studies' table. We also made contact with the authors of included studies, experts in the field of implementation science and key organisations to identify any relevant ongoing or unpublished studies or grey literature publications.

\section{Data collection and analysis}

\section{Selection of studies}

Two review authors (from a pool of three authors: JJ, CB and MF) independently screened abstracts and titles. Review authors were not blind to the author or journal information. We conducted the screening of studies using a standardised screening tool developed based on the Cochrane Handbook for Systematic Reviews of Interventions (Higgins 2019), which we piloted before use. We obtained the full texts of manuscripts for all potentially eligible studies for further examination. For all studies, we recorded information regarding the primary reason for exclusion and documented this in the 'Characteristics of excluded studies' table. We included the remaining eligible studies in the review. We resolved discrepancies between review authors regarding study eligibility by consensus. In instances where the study eligibility could not be resolved via consensus, a third review author made a decision.

\section{Data extraction and management}

Two review authors (from a pool of five authors: JJ, MF, RW, AG and CB), unblinded to author and journal information, independently extracted information from the included studies. We recorded the information extracted from the included studies in a data extraction form that we developed based on the recommendations of the Cochrane Public Health Group Guide for Developing a Cochrane Protocol (Cochrane Public Health Group 2011). We piloted the data extraction form before the initiation of the review. We resolved data extraction discrepancies between review authors by consensus and, where required, via a third review author.

We extracted the following information:

1. Study eligibility as well as the study design, date of publication, childcare service type, country, the demographic/ socioeconomic characteristics of services and participants, the number of experimental conditions, and information to undertake an assessment of study risk of bias.

2. Characteristics of the implementation strategy, including the duration, number of contacts, description of implementation strategies, theoretical underpinning of the strategy (if noted in the study), information to allow classification against the EPOC taxonomy, and to enable an assessment of the overall quality of evidence using the Grades of Recommendation, Assessment, Development and Evaluation (GRADE) approach, as well as data describing consistency of the execution of the intervention with a planned delivery protocol.

3. Study primary and secondary outcomes, including the data collection method, validity of measures used, effect size and measures of outcome variability.

4. Source(s) of research funding and potential conflicts of interest.

\section{Assessment of risk of bias in included studies}

\section{Overall risk of bias}

Within each included study two review authors (MK and FT) assessed risk of bias independently for each review outcome using the 'Risk of Bias' tool described in the Cochrane Handbook for Systematic Reviews of Interventions (Higgins 2011). We determined an overall risk of bias ('high', 'low' or 'unclear') for individual studies and outcomes. For each included study, we assessed risk of bias as 'high', 'low' or 'unclear' for the following domains: sequence generation, allocation concealment, blinding of participants and personnel, blinding of outcome assessment, incomplete outcome data, selective outcome reporting, and 'other' potential sources of bias. We included an additional domain 'potential confounding' to assess the risk of bias in nonrandomised trial designs (Higgins 2011). Confounding was defined as the risk that an 'unmeasured characteristic' shared by those allocated to receive the implementation intervention (or implementation strategy), rather than the intervention itself, was responsible for reported outcomes (Bilandzic 2016). We also included additional domains for cluster-randomised controlled trials, which assessed 'recruitment to cluster', 'baseline imbalance', 'loss of clusters', 'incorrect analysis' and 'compatibility with individually randomised controlled trials' (Higgins 2011). Where required, a third review author adjudicated discrepancies regarding the risk of bias that could not be resolved via consensus (LW). We documented the risk of bias of the included studies in 'Risk of Bias' tables.

We made an overall 'Risk of bias' assessment for an outcome within a study (across domains). As the nature of the experimental manipulations of studies of implementation strategies is such that blinding of participants and personnel is unlikely to be possible, we classified outcomes within a study as at an overall 'high risk' when the study was judged to be at high risk of bias for that outcome on more than one of the following: sequence generation (selection bias), allocation sequence concealment (selection bias), incomplete outcome data (attrition bias), and, in instances where self-report measures of outcome were employed, blinding of outcome assessment. We assigned a low risk of bias to a study when the study was judged to be at low risk of bias for a study outcome on all key criteria.

We also assessed risk of bias for an outcome across studies. Consistent with other Cochrane reviews of public health interventions (Virgara 2019), we judged an outcome as i) low risk if most information for the outcome was generated from studies at low risk of bias ii) unclear risk of bias if most information was from studies at low or unclear risk of bias; or iii) high risk of bias if the proportion of information from studies at high risk of bias was sufficient to affect the interpretation of results.

\section{Measures of treatment effect}

We were able to undertake meta-analysis for implementation outcomes given there was a sufficient number of studies considered suitably homogenous. For binary outcomes, we calculated the standard estimation of the risk ratio (Odds ratio) and a $95 \%$ confidence interval. For continuous data, we calculated a standardised mean difference (SMD), given use of different continuous outcome measures reported in the included studies. We interpreted the magnitude of effect size using the benchmarks suggested by Cohen, considering an SMD of 0.2 a small effect; 0.5 
a medium effect; and 0.8 a large effect (Cohen 1988). We have described all other secondary outcomes narratively.

\section{Unit of analysis issues}

\section{Clustered studies}

We examined clustered studies for unit of analysis errors and recorded these if they occurred in the 'Risk of Bias' tables. No studies included in meta-analysis of implementation outcomes used clustered designs. These designs, however, were utilised in the assessment of individual level child outcomes such as measures of effect on child diet or physical activity.

\section{Dealing with missing data}

We contacted authors of included studies to provide additional information if any outcome data were unclear or missing. All information received was included in the results of the review. We noted any instances of potential selective or incomplete reporting of outcome data in the 'Risk of Bias' tables. We performed metaanalysis using an intention-to-treat principle. Missing data did not preclude inclusion of any studies in meta-analysis, and as such, the potential impact of missing data on the pooled estimates of intervention effects were not investigated in sensitivity analysis.

\section{Assessment of heterogeneity}

For studies included in meta-analysis, we explored heterogeneity via forest plots and the $I^{2}$ statistic (Higgins 2019). We described study participants, intervention, outcomes, and comparators of all included studies in the results and documented such information in the 'Characteristics of included studies' table.

\section{Assessment of reporting biases}

The comprehensive search strategy for this review helped to reduce the risk of reporting bias. We also conducted comparisons between published reports and study protocols, and trial registers, where such reports were available. Instances of potential reporting bias were documented in the 'Risk of Bias' tables.

\section{Data synthesis}

Two authors (CB, LW) were responsible for entering data into Review Manager 5 (RevMan 5) software. Where studies with suitable data were identified, we performed meta-analysis using a randomeffects model in RevMan 5. Meta-analysis was undertaken using the generic inverse variance method. We did not pool data from randomised and nonrandomised trial designs. Similarly, we did not attempt to pool data from nonrandomised studies of different study designs. We reported measures of treatment effect from included studies that were adjusted for potential confounding variables over reported estimates that were not adjusted for potential confounding. Where studies used multiple follow-up periods, we used data from the final (most recent) study followup. We included data from the primary implementation outcome in meta-analyses. In instances where the authors of included studies did not identify a primary implementation outcome, we used the outcome on which the study sample size and power calculation was based. In its absence, for studies using score-based measures of implementation, and reporting total and subscale scores, we assumed the total score represented the primary implementation outcome. Otherwise, we attempted to calculate a relative effect size for each implementation outcome measure, rank these based on effect size and used the measure reporting the median effect size to include in any pooled analysis. We calculated the effect size by subtracting the change from baseline of the primary implementation outcome for the control or comparison group from the change from baseline in the experimental or intervention group. If data to enable calculation of the change from baseline were unavailable, we used the differences between groups post-intervention. For score-based measures, we calculated a standardised ('d') measure of effect size for each outcome to rank the effect size. Where there were an even number of implementation outcomes, one of the two measures at the median was randomly selected and used for inclusion in meta-analysis. We reverse scored implementation measures that did not represent an improvement (for example, the proportion of services without a nutrition policy).

We synthesised findings by outcome, and within the study, synthesised effects by comparison. We included a 'Summary of intervention, measures and absolute intervention effect size table', where we reported the employed implementation strategies classified using the EPOC taxonomy (EPOC 2015), the comparison, the primary implementation outcome measures, the effect sizes on these measures (or median effect size and range of effects where multiple measures of the same outcome are reported) for each study (Table 1).

We included a 'Summary of findings' table to present the key findings of the review (Summary of findings for the main comparison). We generated the table based on the recommendations of the Cochrane Handbook for Systematic Reviews of Interventions and the EPOC Group and included i) a list of all primary and secondary outcomes in the review, ii) a description of intervention effect, iii) the number of participants and studies addressing each outcome, and iv) a grade for the overall quality of the body of evidence for each outcome. In particular, the table provides key information concerning the quality of evidence, the magnitude of the effect of the interventions examined, and the sum of available data on the main outcomes. 'Summary of findings' tables were produced using data from randomised controlled trials only as the included nonrandomised trials did not provide greater certainty evidence, nor did they include outcomes that were not also reported in included randomised trials. Similarly, 'Summary of findings' tables were produced for studies reporting the effects of interventions versus usual care or a minimal support comparison group, as this was considered of primary interest to end-users.

Two review authors $(\mathrm{CB}, \mathrm{RH})$ rated the overall quality of evidence for each outcome using the GRADE system (Guyatt 2010), with any disagreements resolved via consensus or, where required, by a third review author (LW). The GRADE system defines the quality of the body of evidence for each review outcome regarding the extent to which one can be confident in the review findings. The GRADE system required an assessment of methodological quality, directness of evidence, heterogeneity, precision of effect estimates, and risk of publication bias. We used the GRADE quality ratings (from 'very low' to 'high') to describe the quality of the body of evidence for each review outcome and we included these in 'Summary of findings for the main comparison'. We assessed the quality of evidence separately for randomised and non-randomised trials. Where there were multiple measures of the same outcome, we assessed the quality of evidence for each measure separately. In such instances, we selected the measure of the outcome with the greatest collective (across study) sample size to present in the 
'Summary of findings' tables to represent the GRADE assessment of that outcome. However, we also noted the GRADE assessments of other measures of the outcome as comments in the 'Summary of findings' table for completeness.

\section{Subgroup analysis and investigation of heterogeneity}

In the published protocol (Wolfenden 2015b), subgroup analyses and box plots were planned to explore heterogeneity if the $1^{2}$ value was greater than $75 \%$. As measures of heterogeneity did not reach this threshold, subgroup analyses were not undertaken. Nonetheless, clinical and methodological heterogeneity of included studies was described narratively. To describe the impact of implementation strategies delivered 'at scale' (defined as involving 50 or more childcare services), we performed subgroup analyses narratively for the primary implementation outcomes. Specifically, this was undertaken for included studies that sought to improve implementation of policies, practices or programmes across 50 or more services.

\section{Sensitivity analysis}

Sensitivity analysis was planned by removing studies with a high risk of bias and by removing outliers contributing to statistical heterogeneity following visual inspection of the forest plots (i.e. where the confidence intervals of a study did not overlap with other included studies). However, none of the studies included in metaanalysis were judged to be at high risk of bias, nor were outliers identified following inspection of forest plots.

\section{Summary of findings and assessment of the certainty of the} evidence

\section{RE S U L T S}

\section{Description of studies}

See Characteristics of included studies; Characteristics of excluded studies; Characteristics of ongoing studies

\section{Results of the search}

The electronic search for this update, conducted on 22 February 2019, yielded 3236 citations (Figure 1). We identified an additional 1093 records from handsearching key journals and checking reference lists of included studies. We identified no additional records through our contact with the authors of included studies, experts in the field of implementation science and key organisations. Following screening of titles and abstracts, we obtained the full texts of 71 manuscripts for further review, of which we included as part of this update 21 manuscripts describing 11 individual studies. We contacted the authors of four included studies to provide additional information where any outcome data were unclear or missing. All information received by authors was included in the results of the review. As 10 studies were included in the original version of this review (Alkon 2014; Bell 2014; Benjamin 2007; Finch 2012; Finch 2014; Gosliner 2010; Hardy 2010; Johnston Molloy 2013; Ward 2008; Williams 2002), this update brought the total number of included studies to 21 studies. Additionally, 11 studies were identified as ongoing studies through searches of clinical trial registration databases that have not yet been published. 
Figure 1. Study flow diagram.

\begin{tabular}{|l|}
10 studies \\
included in the \\
original review \\
synthesis
\end{tabular}

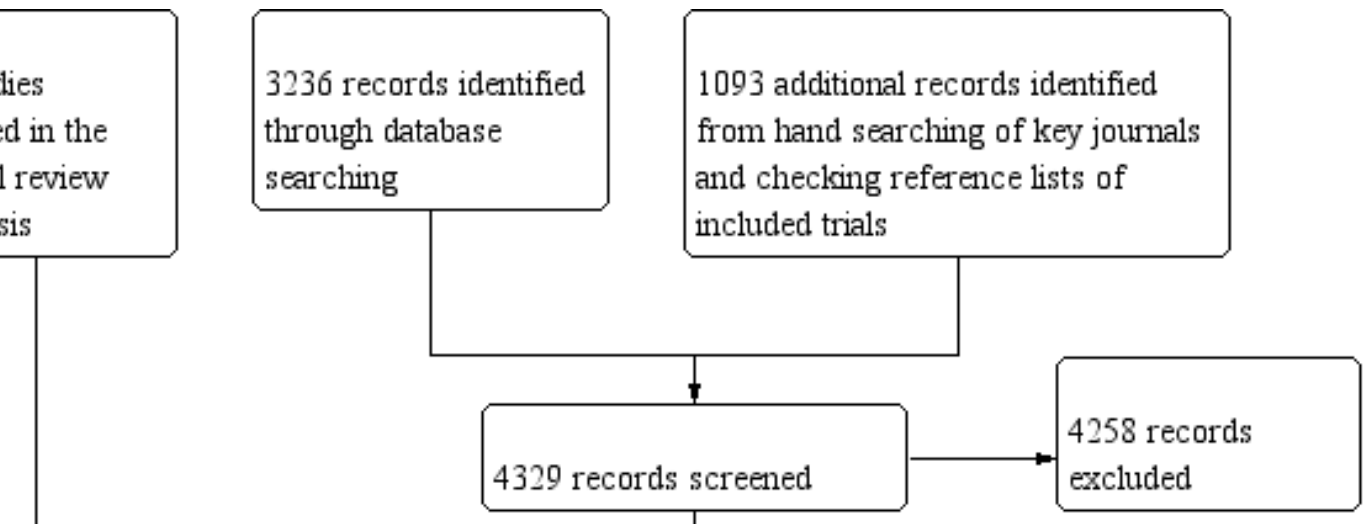

39 full-text articles excluded, with reasons:

Participants: $\mathrm{n}=2$

Intervention: $\mathrm{n}=1$

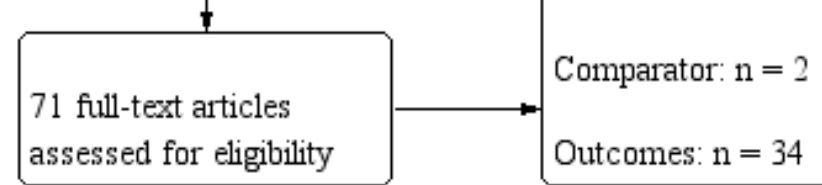

$11 \mathrm{NEW}$ studies (21

manuscripts) included in

review synthesis

11 studies classified as ongoing

TOTAL 21 studies (38 articles)

included in qualitative synthesis

Strategies to improve the implementation of healthy eating, physical activity and obesity prevention policies, practices or programmes 


\section{Included studies}

\section{Types of studies}

The included studies were predominantly conducted in the USA ( $n=12$ ) (Alkon 2014; Benjamin 2007; Esquivel 2016; Gosliner 2010; Mazzucca 2017; Morshed 2016; O'Neill 2017; Sharma 2018; Stookey 2017; Ward 2008; Ward 2017; Williams 2002) and Australia ( $=8$ ) (Bell 2014; Finch 2012; Finch 2014; Finch 2019; Hardy 2010; Jones 2015; Seward 2017; Yoong 2016), but there was also one study from Ireland (Johnston Molloy 2013). Studies were conducted between 1995 and 2018, although two studies did not report the years of data collection (Benjamin 2007; Gosliner 2010). There was evidence of some heterogeneity in the participants, interventions, outcomes and study design characteristics of included studies. All but one included study (Mazzucca 2017) reported receiving funding support to undertake the study. Funding support for such studies were from government or charitable foundations. No industry funding was reported.

\section{Participants}

Of the 21 included studies, 15 recruited childcare services located in disadvantaged areas or specifically serving disadvantaged, lowincome or minority children (Alkon 2014; Bell 2014; Esquivel 2016; Finch 2012; Finch 2014; Gosliner 2010; Johnston Molloy 2013; Jones 2015; Morshed 2016; O’Neill 2017; Seward 2017; Sharma 2018; Stookey 2017; Ward 2017; Williams 2002). The socioeconomic characteristics of the service locality or children attending the childcare services were not described in the remaining six studies. The number of childcare services participating in the studies included in the review varied. The largest study recruited 583 childcare services (preschools) (Bell 2014), and a further eight studies sought to improve implementation of policies, practices or programmes in 50 or more services (Finch 2012; Finch 2019; Johnston Molloy 2013; Jones 2015; O'Neill 2017; Seward 2017; Ward 2008; Yoong 2016). Six studies recruited between nine and 20 services (Alkon 2014; Benjamin 2007; Finch 2014; Gosliner 2010; Morshed 2016; Williams 2002). Twelve of the 21 included studies were conducted in high-income countries by two research groups in the USA and Australia (Alkon 2014; Bell 2014; Benjamin 2007; Finch 2012; Finch 2014; Finch 2019; Jones 2015; Mazzucca 2017; Seward 2017; Ward 2008; Ward 2017; Yoong 2016).

\section{Interventions}

Six studies targeted the implementation of healthy eating policies or practices only (Bell 2014; Morshed 2016; Seward 2017; Ward 2017; Williams 2002; Yoong 2016), three targeted the implementation of physical activity policies and practices only (Finch 2012; Finch 2014; Mazzucca 2017), and 12 targeted both healthy eating and physical activity policies and practices (Alkon 2014; Benjamin 2007; Esquivel 2016; Finch 2019; Gosliner 2010; Hardy 2010; Johnston Molloy 2013; Jones 2015; O’Neill 2017; Sharma 2018; Stookey 2017; Ward 2008).

All studies used multiple implementation strategies, with the exception of one study (Yoong 2016). The strategies tested across studies examined only a small number of those described in the EPOC taxonomy that could be applied to improve implementation in the setting. The definitions of each of the EPOC subcategories used to classify implementation strategies employed by studies included in the review are provided in Table 2. Using the EPOC taxonomy descriptors for tested implementation strategies, 17 of the 21 studies tested educational meetings and educational materials (Alkon 2014; Bell 2014; Benjamin 2007; Esquivel 2016; Finch 2012; Finch 2014; Gosliner 2010; Hardy 2010; Johnston Molloy 2013; Jones 2015; Mazzucca 2017; Morshed 2016; Seward 2017; Stookey 2017; Ward 2008; Ward 2017; Williams 2002). The remaining studies testing educational meetings and educational materials in combination with other strategies such as audit and feedback (Alkon 2014; Bell 2014; Johnston Molloy 2013; Jones 2015; Seward 2017; Stookey 2017; Ward 2017), educational outreach visits or academic detailing (Benjamin 2007; Gosliner 2010; Hardy 2010; Jones 2015; Mazzucca 2017; Seward 2017; Stookey 2017 Ward 2008), small incentives (Gosliner 2010; Hardy 2010; Stookey 2017; Williams 2002) or opinion leaders (Bell 2014; Finch 2012; Finch 2019; Seward 2017).

Twelve studies reported that strategies to support implementation were theoretically based (Bell 2014; Benjamin 2007; Esquivel 2016; Finch 2014; Finch 2019; Jones 2015; Mazzucca 2017; Morshed 2016; Seward 2017; Sharma 2018; Ward 2008; Yoong 2016). The theories adopted in these studies included components of social cognitive theory (Benjamin 2007; Mazzucca 2017; Sharma 2018; Ward 2008), practice change and capacity building theoretical frameworks (Bell 2014), theory of planned behaviour (Yoong 2016), consolidated framework for implementation research (Finch 2019; Jones 2015), theoretical domains framework (Seward 2017) and social-ecological models of health behaviour change (Esquivel 2016; Finch 2014; Morshed 2016).

Intervention duration for the included studies ranged from six to eight weeks (Yoong 2016) to three years (Williams 2002). The duration of the majority of interventions were six to 12 months (Alkon 2014; Benjamin 2007; Esquivel 2016; Finch 2014; Finch 2019; Gosliner 2010; Jones 2015; O'Neill 2017; Seward 2017; Stookey 2017; Ward 2008) and four studies had a duration of longer than 12 months (Bell 2014; Morshed 2016; Sharma 2018; Williams 2002).

\section{Outcomes}

A variety of implementation outcome measures were used to assess the implementation strategies across included studies. Nineteen studies included continuous measures of implementation outcomes including policy or environment scores (Alkon 2014; Benjamin 2007; Esquivel 2016; Finch 2019; Johnston Molloy 2013; Jones 2015; Mazzucca 2017; O’Neill 2017; Seward 2017; Sharma 2018; Ward 2008; Ward 2017), minutes of policy or programme implementation (Finch 2012; Finch 2014; Hardy 2010), frequency of policy or programme implementation (Finch 2014; Hardy 2010), or quantity of food or beverages or macronutrients provided to children (Bell 2014; Morshed 2016; Williams 2002; Yoong 2016).

Eleven studies reported a dichotomous measure of implementation, each of which reported the percentage of staff or childcare services that implemented a policy, practice or programme (Alkon 2014; Bell 2014; Finch 2012; Finch 2014; Finch 2019; Gosliner 2010; Hardy 2010; Jones 2015; O’Neill 2017; Seward 2017; Stookey 2017).

Implementation was primarily assessed using telephone interviews or surveys/questionnaires completed by childcare service staff (Bell 2014; Benjamin 2007; Finch 2012; Finch 2019; Gosliner 2010; Hardy 2010; Jones 2015; Mazzucca 2017; Seward 2017; Sharma 2018; Ward 2017; Yoong 2016), audits of service documents conducted by researchers (Bell 2014; Seward 2017; 
Williams 2002) or by direct observation (Alkon 2014; Esquivel 2016; Finch 2014; Johnston Molloy 2013; Morshed 2016; O’Neill 2017; Stookey 2017; Ward 2008).

The validity of six of the ten studies utilising survey/questionnaire based instruments to assess implementation was not reported (Bell 2014; Finch 2012; Finch 2019; Gosliner 2010; Hardy 2010; Sharma 2018). Outcome assessments were conducted at various time points following intervention completion. Four studies conducted outcome assessments immediately following intervention completion (Jones 2015; Mazzucca 2017; Morshed 2016; Seward 2017), whilst other studies included follow-up assessments of five months (Hardy 2010) to four years following intervention completion (Johnston Molloy 2013).

Nine studies included child behavioural or weight-related outcomes (Alkon 2014; Esquivel 2016; Finch 2014; Jones 2015; Mazzucca 2017; Seward 2017; Sharma 2018; Stookey 2017; Williams 2002). Of the nine studies, four measured child diet (Jones 2015; Seward 2017; Sharma 2018; Williams 2002), five measured child physical activity (Alkon 2014; Finch 2014; Jones 2015; Mazzucca 2017; Sharma 2018) and five measured child weight status (Alkon 2014; Esquivel 2016; Sharma 2018; Stookey 2017; Williams 2002).

Three of the 21 included studies reported on potential adverse effect outcomes, which included negative feedback received by the childcare service (Seward 2017) and occurrence of child injury (Finch 2014; Jones 2015). Eight studies included a measure of acceptability (Benjamin 2007, Finch 2012, Finch 2014, Finch 2019, Hardy 2010, Jones 2015, Mazzucca 2017, Ward 2017), and 12 studies measured penetration of the intervention and implementation strategies (Alkon 2014; Finch 2012; Finch 2014; Hardy 2010; Gosliner 2010; Johnston Molloy 2013; Jones 2015; Mazzucca 2017; Seward 2017; Stookey 2017; Ward 2008; Yoong 2016). None of the 21 studies reported intervention costs or cost-effectiveness analyses.

\section{Study design characteristics}

Sixteen of the included studies were randomised trials (or clusterrandomised trials) (Alkon 2014; Benjamin 2007; Esquivel 2016;
Finch 2014; Finch 2019; Gosliner 2010; Hardy 2010; Johnston Molloy 2013; Jones 2015; Mazzucca 2017; Morshed 2016; Seward 2017; Stookey 2017; Ward 2008; Ward 2017; Yoong 2016), and five were nonrandomised trials with a parallel control group (Bell 2014; Finch 2012; O’Neill 2017; Sharma 2018; Williams 2002).

Nineteen studies compared an implementation strategy to usual practice or minimal support control (Alkon 2014; Bell 2014; Benjamin 2007; Esquivel 2016; Finch 2012; Finch 2014; Finch 2019; Hardy 2010; Jones 2015; Mazzucca 2017; Morshed 2016; O’Neill 2017; Seward 2017; Sharma 2018; Stookey 2017; Ward 2008; Ward 2017; Williams 2002; Yoong 2016). Two studies directly compared two different implementation strategies (Gosliner 2010; Johnston Molloy 2013).

\section{Excluded studies}

Thirty-nine studies were excluded following review of 71 full texts (Figure 1) for the following reasons: participants $n=2$; intervention $n=1$; comparator $n=2$; outcomes $n=34$. We excluded a study based on 'inappropriate outcomes' if it: did not measure implementation outcomes, did not measure implementation outcomes for both intervention and control groups, or did not measure betweengroup differences in implementation outcomes.

\section{Risk of bias in included studies}

See Characteristics of included studies.

For the primary implementation outcomes, 'Risk of bias' assessment for each criterion for each study is presented in Figure 2 and summarised within the Characteristics of included studies tables. Figure 3 illustrates the overall risk of bias of each study for primary implementation outcomes (across all domains). 'Risk of bias' assessments are described in detail below. Risk of bias assessments for secondary outcomes of each study are presented in Appendix 2. 
Figure 2. 'Risk of bias' summary: review authors' judgements about each risk of bias item for each included study.

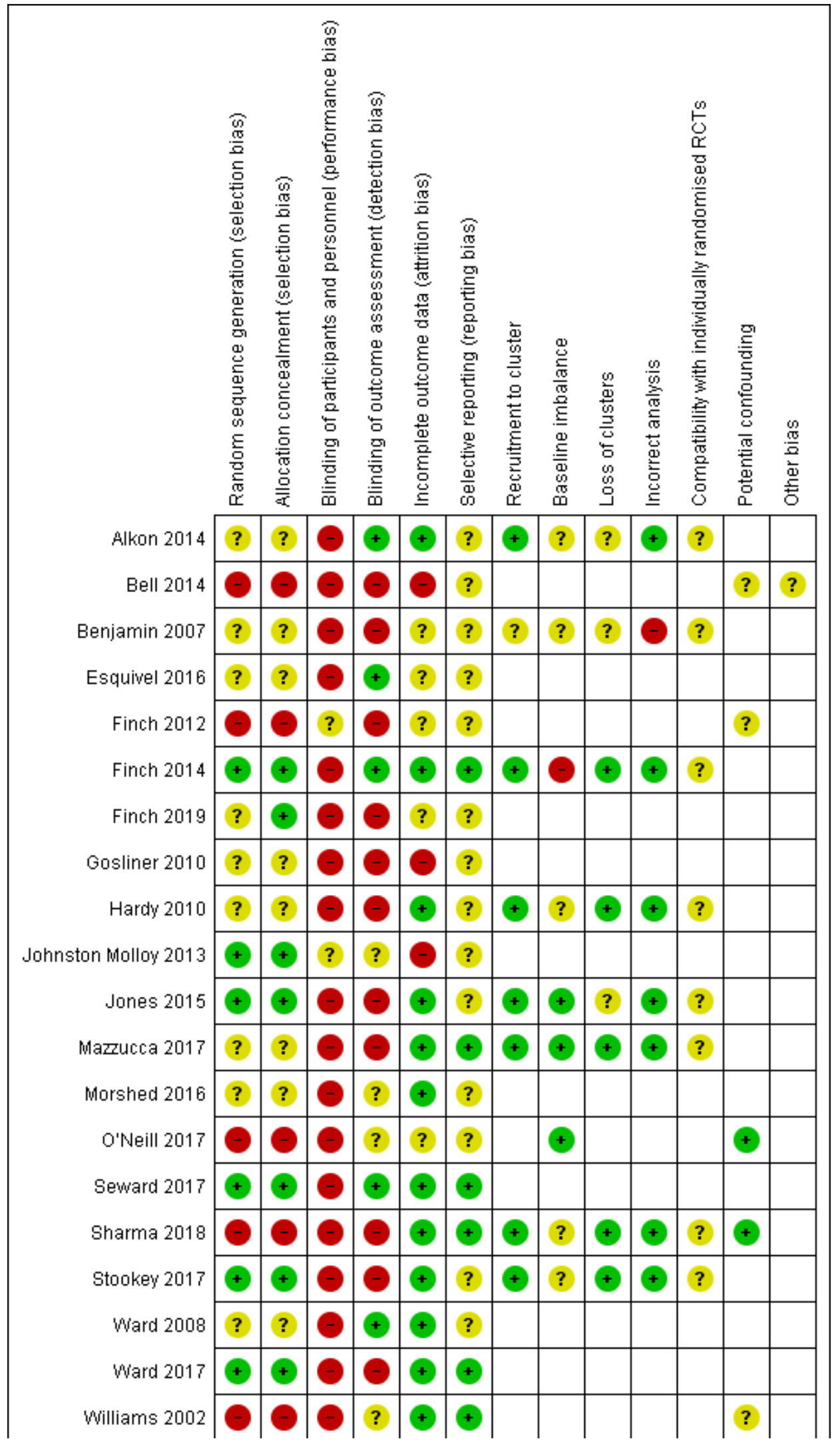

Strategies to improve the implementation of healthy eating, physical activity and obesity prevention policies, practices or programmes 
Figure 2. (Continued)

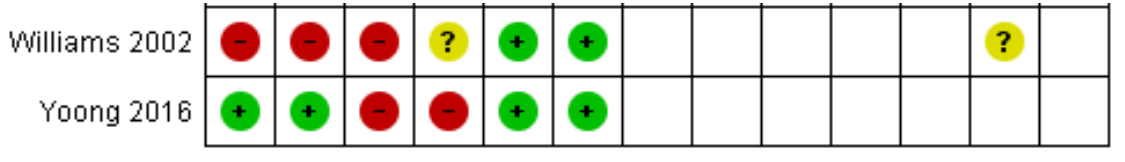

Figure 3. 'Risk of bias graph': review authors' judgements about each risk of bias item presented as percentages across all included studies.

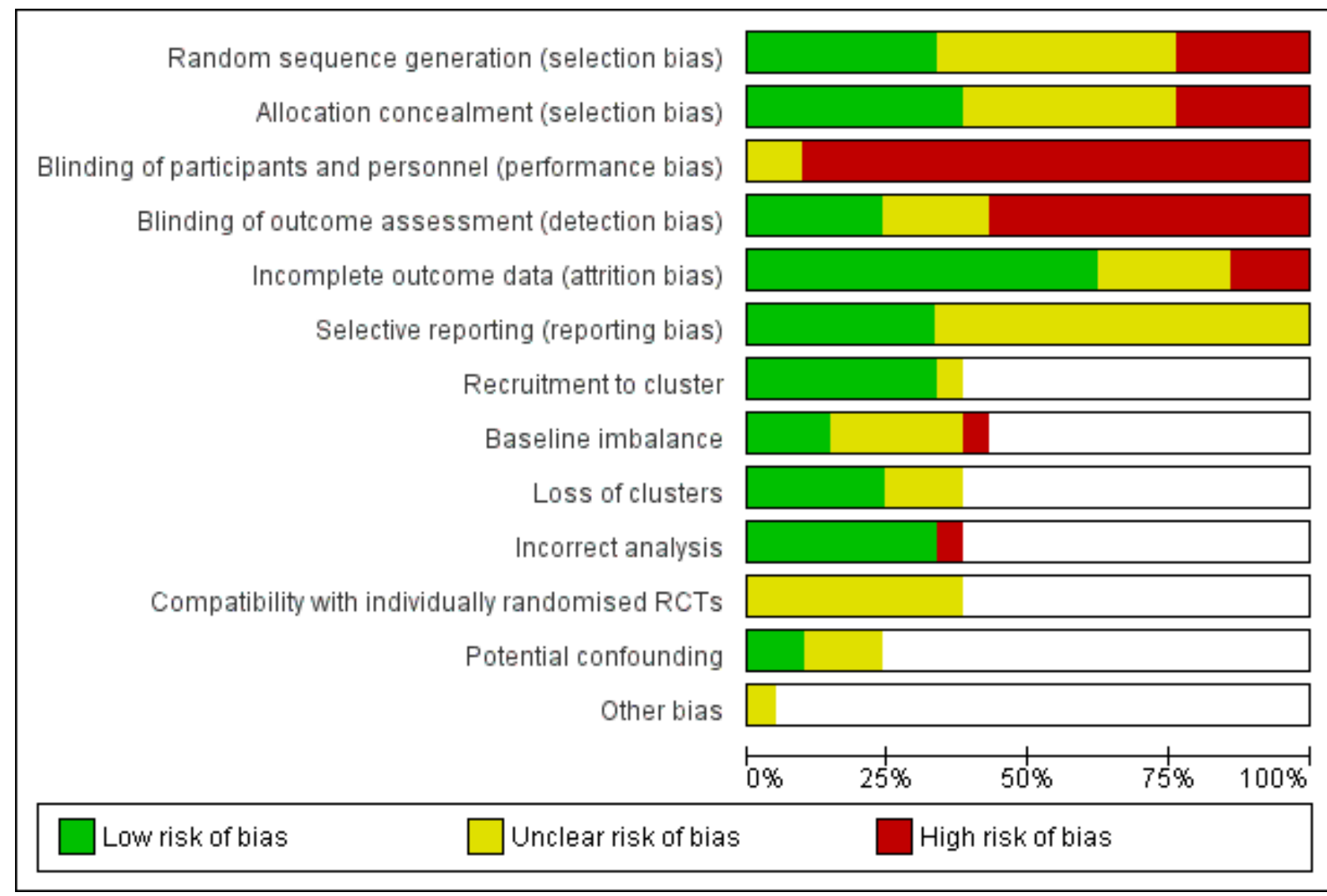

\section{Allocation}

Risk of selection bias differed across studies. Seven studies were low risk as computerised random number functions or tables were used to generate random sequences and allocation was undertaken automatically in a single batch, preventing allocation from being pre-empted (Finch 2014; Johnston Molloy 2013; Jones 2015; Seward 2017; Stookey 2017; Ward 2017; Yoong 2016). While the study conducted by Finch and colleagues (Finch 2019) also undertook these procedures, participating services were removed following randomisation and it is unclear whether this affected the randomisation. For the five studies with non-randomised designs, the risk of selection bias was high (Bell 2014; Finch 2012; O'Neill 2017; Sharma 2018; Williams 2002). For the remaining eight studies, such bias was unclear as these studies did not report on methods for sequence generation or allocation.

\section{Blinding}

For almost all studies $(n=19)$, the risk of performance bias was high due to participants and research personnel not being blind to group allocation. For the remaining two studies, the risk of performance bias was unclear as in both studies the control group also received some form of intervention (Finch 2012; Johnston Molloy 2013). Detection bias differed across studies based on whether outcome measures were objective (low risk) or self-reported (high risk), and whether research personnel were blind to group allocation when conducting outcome assessment (low risk). For five studies, the risk of detection bias was low (Alkon 2014; Esquivel 2016; Finch 2014; Seward 2017; Ward 2008). For the remainder of the studies, the risk of detection bias was either high $(n=12)$ or unclear $(n=4)$ due to insufficient information on whether data collection staff were blind to group allocation.

\section{Incomplete outcome data}

For just over half the studies ( $n=13$ ), the risk of attrition bias was low as either all or most participating services were followed up and/or sensitivity analysis was conducted to assess the impact of missing data (Alkon 2014; Finch 2014; Hardy 2010; Jones 2015; Mazzucca 2017; Morshed 2016; Seward 2017; Sharma 2018; Stookey

Strategies to improve the implementation of healthy eating, physical activity and obesity prevention policies, practices or programmes 
2017; Ward 2008; Ward 2017; Williams 2002; Yoong 2016). For two studies, the risk of such bias was high due to a large difference in the proportion of participating services lost to follow-up between groups (Bell 2014; Johnston Molloy 2013). Risk of attrition bias was also high for the study conducted by Gosliner and colleagues, as participants who did not complete the intervention were excluded from the analysis (Gosliner 2010). For the remaining studies, the risk of attrition bias was unclear as insufficient information was provided regarding the treatment of missing data.

\section{Selective reporting}

For fourteen studies, a published protocol paper or trial registration record was not identified and therefore it was unclear whether reporting bias had occurred. For seven studies, the risk of reporting bias was low as protocol papers were available and all a priori determined outcomes were reported (Finch 2014; Mazzucca 2017; Seward 2017; Sharma 2018; Ward 2017; Williams 2002; Yoong 2016). For the study conducted by Esquivel and colleagues, risk of reporting bias was also unclear as there were secondary outcomes listed in a protocol paper that did not appear to have been reported (Esquivel 2016).

\section{Other potential sources of bias}

For the eight studies that were cluster-randomised controlled trials, we assessed the potential risk of additional biases (Alkon 2014; Benjamin 2007; Finch 2014; Hardy 2010; Jones 2015; Mazzucca 2017; Sharma 2018; Stookey 2017).

For the potential risk of recruitment (to cluster) bias, seven studies were low risk as either a random or census approach was used for recruitment (Alkon 2014; Finch 2014; Hardy 2010; Jones 2015; Mazzucca 2017; Sharma 2018; Stookey 2017). For the remaining study (Benjamin 2007), it was unclear if such bias existed due to insufficient detail regarding participant recruitment.

Regarding risk of bias due to baseline imbalances, for five studies the risk was unclear (Alkon 2014; Benjamin 2007; Hardy 2010; Sharma 2018; Stookey 2017). One study was at high risk due to baseline imbalances in service characteristics, with no mention of adjustments within the analysis (Finch 2014) and two studies were at low risk due to no baseline imbalances (Jones 2015; Mazzucca 2017).

Five studies were at low risk for loss of clusters as either all children were followed up or there was no loss of clusters (Finch 2014; Hardy 2010; Mazzucca 2017; Sharma 2018; Stookey 2017). For two studies, risk of bias was unclear due to insufficient information regarding the treatment of clusters that were lost and the impact of this loss (Alkon 2014; Benjamin 2007). For the study conducted by Jones 2015, risk of bias was also unclear as follow-up data were only collected from a random sample of clusters.

For risk of bias due to incorrect analysis, almost all studies ( $\mathrm{n}=$ 7) were low risk (Alkon 2014; Finch 2014; Hardy 2010; Jones 2015; Mazzucca 2017; Sharma 2018; Stookey 2017), while the remaining study was high risk as no statistical analysis was undertaken due to the small sample size (Benjamin 2007).

For all eight cluster-randomised controlled trials, risk of bias in regards to compatibility with individually randomised controlled trials was unable to be determined (Alkon 2014; Benjamin 2007;
Finch 2014; Hardy 2010; Jones 2015; Mazzucca 2017; Sharma 2018; Stookey 2017).

For the five studies with nonrandomised designs (Bell 2014; Finch 2012; O'Neill 2017; Sharma 2018; Williams 2002), we also considered the potential risk of bias due to confounding factors. For three studies (Bell 2014; Finch 2012; Williams 2002), it was unclear whether confounders were adjusted for. For the remaining two studies (O'Neill 2017; Sharma 2018), known confounders were adequately adjusted for in the outcome analysis.

\section{Risk of bias for an outcome within a study (across domains)}

For implementation outcomes, six studies were judged to be at overall high risk of bias (Bell 2014; Finch 2012; Gosliner 2010; O'Neill 2017; Sharma 2018; Williams 2002) and two at low risk of bias (Finch 2014; Seward 2017). The remaining 13 studies were judged to be at an unclear overall risk of bias. Of the five studies reporting physical activity outcomes, one study was at overall high risk (Sharma 2018), two at low risk (Finch 2014; Jones 2015) and the remaining at unclear risk of bias (Alkon 2014; Mazzucca 2017). Of the four studies reporting dietary intake outcomes, two were at low risk of bias (Jones 2015; Seward 2017) and two were at high risk of bias (Sharma 2018; Williams 2002). Of studies reporting measures of child weight status, one study was judged to be at high risk of bias (Williams 2002), three studies (Alkon 2014; Esquivel 2016; Sharma 2018) were judged to be at unclear risk of bias and one study (Stookey 2017) was judged as at low risk of bias for those outcomes. Finally, all three studies reporting adverse events were judged to be at unclear risk of bias for those outcomes (Finch 2014; Jones 2015; Seward 2017).

\section{Risk of bias for an outcome across studies}

Across included studies, implementation outcomes, physical activity outcomes, weight status outcomes and adverse outcomes were assessed as being at unclear risk of bias, while dietary intake outcomes were assessed as being at high risk of bias.

\section{Effects of interventions}

See: Summary of findings for the main comparison

See Summary of findings for the main comparison; Table 1.

\section{Studies comparing a strategy to improve the implementation of any healthy eating, physical activity or obesity prevention policy, practice or programme in centre-based childcare services compared with no intervention, 'usual' practice or minimal support control}

\section{Continuous outcomes}

\section{Score-based measures of implementation}

Score-based measures of implementation were the most common continuous outcomes in studies comparing an implementation strategy with usual practice or minimal support control and were reported in 11 studies including nine randomised trials. Pooled analysis providing moderate-certainty evidence including all nine randomised trials with score-based measures of implementation (Alkon 2014; Benjamin 2007; Esquivel 2016; Finch 2019; Jones 2015; Mazzucca 2017; Seward 2017; Ward 2008; Ward 2017) reported an improvement (SMD 0.49; 95\% $\mathrm{Cl} 0.19$ to $0.79 ; \mathrm{I}^{2}=54 \%$; $\mathrm{P}<0.001$; participants $=495$ services; equivalent to a mean difference of 0.88 on the Environment and Policy Assessment and Observation

Strategies to improve the implementation of healthy eating, physical activity and obesity prevention policies, practices or programmes 
(EPAO) scale) favouring groups receiving implementation support strategies (Analysis 1.1). Visual inspection of funnel plots suggested the potential for publication bias. Sensitivity analysis was not performed as none of the randomised trials contributing to the meta-analysis were assessed as being at high risk of bias for these outcomes. As an 12 value greater than $75 \%$ was set in the protocol a priori as the threshold for investigating heterogeneity, and owing to the relatively small number of included trials in the pooled analysis, statistical heterogeneity was not explored quantitatively.

The two nonrandomised trials using score-based measures of implementation both reported positive improvements in implementation (O'Neill 2017; Sharma 2018) (participants $=79$ childcare services, two studies, very low-certainty evidence). The first, a nonrandomised trial conducted in the USA by Neelon and O'Neill in 2017, evaluated the impact of the introduction of new physical activity standards on childcare services physical activity practices in a sample of 34 South Carolina childcare services, where it was mandatory, in comparison to 30 services located in North Carolina - a state not making such policy changes (O'Neill 2017). The EPAO tool was used by trained researchers to assess physical activity practices and environments prior to and nine months following implementation of the standards. Within South Carolina services, where the mandatory standards were introduced, the total Physical Activity Environment Score increased from 8.6 (standard error, 0.3) to 9.7 (standard error, 0.3) from baseline to follow-up while increasing marginally from 8.9 (standard error, 0.4) to 9.1 (standard error, 0.4) in North Carolina services $(P=0.06)$.

The second, a nonrandomised trial undertaken in the USA in 25 childcare services, examined the impact of an implementation strategy comprised of educational meetings, reminders and academic detailing to improve the implementation of a classroom nutrition curriculum, structured physical activities and distribution of health information to families (Sharma 2018). A score-based measure (a per cent implementation index) using data collected from teacher surveys was used to assess improvement in programme implementation. The study reported improvements in implementation favouring services allocated to receive an implementation strategy (mean difference 15.17, $\mathrm{P}=0.002$ ).

\section{Time or frequency-based measures}

Three studies reported minutes of policy or programme implementation (Finch 2012; Finch 2014; Hardy 2010) or frequency of policy or programme implementation (Finch 2014; Hardy 2010; low-certainty evidence), the findings of which were mixed.

Two of the three studies were randomised trials (participants $=49$ services, two studies, low-certainty evidence). The first, undertaken by Hardy and colleagues was a cluster-randomised trial evaluating the 'Munch and Move' programme in one state of Australia (New South Wales) (Hardy 2010). All 61 government services (preschools) in the study region were invited to participate in the trial, of which 29 consented and were randomised. Services allocated to the implementation support group received educational materials, educational meetings, educational outreach visits and small grants to implement the programme. Those in the comparison group received usual care. To assess policy and practice implementation, interviews with all service managers occurred at baseline and immediately following the five-month intervention. The frequency of service provided in fundamental movement skill activities for children increased from 1.3 sessions per week to 3.2 sessions per week in the intervention group and remained unchanged in control services (difference at follow-up of $1.5,95 \% \mathrm{Cl} 0.01$ to 2.9 , $P=0.05$ ). There were no meaningful differences between groups in the frequency of structured play sessions per week (adjusted difference $0.02,95 \% \mathrm{Cl}-1.5$ to 1.5 ), unstructured play sessions per week (adjusted difference not reported), minutes per session of structured play (adjusted difference $0.09,95 \% \mathrm{Cl}-11.6$ to 11.8 ), unstructured play (adjusted difference $7.7,95 \% \mathrm{Cl}-15.6$ to 31.0 ) and fundamental movement skill sessions (adjusted difference $3.4,95 \% \mathrm{Cl}-9.7$ to 16.5$)$. The effect sizes for differences between groups on any of the four measures of nutrition policy or practice implementation including food-based activities, rules around food and food policies were not presented, although authors states these were non-significant.

The second, by Finch and colleagues, was a randomised controlled trial with 20 centre-based childcare services in New South Wales, Australia (Finch 2014). The intervention primarily sought to determine the effectiveness of a physical activity intervention, implemented by childcare service staff on the physical activity levels of children attending childcare. Secondary outcomes included assessment of the effectiveness of implementation strategies and the impact of the intervention on rates of child injury. The trial reported a difference between groups $(P<0.02)$ in time spent in structured physical activities (intervention services increased from 23.67 (SD \pm 6.03 ) minutes at baseline to 52.40 (SD \pm 45.29) minutes at follow-up; control services decreased from 37.80 (SD \pm 13.33$)$ at baseline to $27.00(S D \pm 1.41)$ at follow-up). There were at times large differences between groups in the number of occasions of fundamental movement skill development activity sessions (intervention +0.8 sessions, control +0.2 sessions), the number of times staff participated in active play (intervention +1.4 times, control -1.6 times); or the number of times staff provided positive statements about physical activity (intervention +1.7 times, control -10.4 times), although the effects were uncertain as confidence intervals crossed the line of no effect ( $P=0.07$ to 0.08 ). The difference between groups in nine other measures of policy and practice implementation including: total minutes of fundamental movement skill development activity sessions, number of times staff prompted physical activity, total minutes of television viewing, total minutes of seated time, or the number of physical activitypromoting resources or equipment were small and uncertain.

The final study, a nonrandomised trial (participants $=392$ services, one study, very low-certainty evidence) examined a strategy to increase implementation of physical activity-promoting policies and practices in centre-based childcare services (Finch 2012). All services located within the Hunter New England geographic area of New South Wales, Australia $(n=338)$ were invited to participate in the intervention and received support to implement a number of policies and practices to promote child physical activity in care. A $10 \%$ sample of services in the rest of the state $(n=268)$ were randomly selected to serve as a comparison group. Services in the comparison region had the opportunity to receive government support to implement 'Munch and Move' (described above), a programme targeting similar policies and practices but utilising a less intensive series of implementation support (Hardy 2010). Implementation of physical activity practices was assessed at baseline and between eight and 12 months post-intervention via a telephone interview administered to service managers. At followup there was no clear difference between groups in time spent in 
structured physical activities (intervention +0.2 hours, control +0.1 hours, $P=0.65$ ).

\section{Quantity of food measures}

Five studies reported quantity of food or beverages or macronutrients provided to children as implementation outcomes (Alkon 2014; Bell 2014; Morshed 2016; Williams 2002; Yoong 2016), the findings of which were mixed.

Three of these studies were randomised trials (participants = 171 services, three studies, low-certainty evidence). Morshed and colleagues conducted a randomised trial of 16 Head Start childcare services in American Indian and predominantly Hispanic communities in rural New Mexico (Morshed 2016). Services allocated to receive implementation support were provided with education materials including eight nutrition curriculum modules intended for implementation over two school years and educational meetings for foodservice staff occurring quarterly and aimed at supporting policy and behavioural changes to food purchasing and menus. Specifically, childcare service staff were supported to implement a range of practices including increasing structured physical activity time, providing opportunities for children to try new fruits and vegetables, and increasing the variety of fruits, vegetables, whole grain foods and low-fat dairy products served to children. Services allocated to the control followed usual classroom activities and did not receive any implementation support from the research team. Data were collected prior to, and immediately following, the two-year implementation period via weighing foods served to children by research staff at participating services. The intervention decreased fat provided through milk (change relative to control $=0.82 ; 95 \% \mathrm{Cl} 0.71$ to $0.94, \mathrm{P}$ value not reported). There was little difference between group servings of fruit, vegetables, whole-grain servings, discretionary fats, and added sugar, with estimates of change in the group allocated to receive implementation support relative to control ranging from $0.94(95 \% \mathrm{Cl} 0.65$ to 1.37$)$ to 1.09 (95\% Cl 0.92 to 1.30$)$ across these measures.

A randomised trial by Alkon and colleagues assessed the impact of an implementation strategy including educational materials, educational meetings and audit and feedback on the types and portions of all foods and beverages served to children in childcare services (Alkon 2014). Assessments were conducted by direct observations conducted by researchers using the Diet Observation in Child Care (DOCC) tool, a validated instrument (Alkon 2014). At follow-up, there was considerable variation between groups on 10 measures of the portions of foods and beverages offered to children at meals and snack time (range $-2.7 \%$ to $133 \%$ ).

In a randomised trial of 77 childcare services, Yoong and colleagues investigated the impact of providing printed educational materials on childcare service cooks provision of fruit and vegetables on their food service menu (Yoong 2016). The educational materials included a mailed two-page education resource and the menu planning checklist and incorporated coloured visuals outlining recommended serving sizes (endorsed by a reputable health promotion organisation). Outcome data assessing serves of fruit and vegetables provided on menus were collected via a telephone interview with childcare service cooks. At follow-up, both services allocated to receive implementation support and those that were not reported providing a mean of 2.9 serves of vegetables on their menus. The mean serves of fruit was higher among services receiving implementation support at follow-up (mean $=3.8, \mathrm{SD}=$ 1.1) compared to the comparison group (mean $=3.3, \mathrm{SD}=0.8 ; \mathrm{P}=$ $0.057)$.

The two nonrandomised trials provided very low-certainty evidence regarding the effects of implementation strategies on measures of food provision (participants $=440$ services, two studies, very low-certainty evidence). In Australia, Bell and colleagues conducted a nonrandomised trial to determine the impact of an implementation intervention to improve healthy eating policies and practices in centre-based childcare services (Bell 2014). All services in one geographic region of the state of New South Wales, Australia (Hunter, New England) were offered the intervention $(n=287)$ and provided implementation support. A random sample of $10 \%$ of childcare services located in all other regions of New South Wales were invited to participate in the evaluation and served as a control group ( $n=296)$. The study was conducted in the context of the 'Good for Kids. Good for Life' programme but occurred over a different period to the study by Finch and colleagues (Finch 2012). Services allocated to the control group received usual care that may have included exposure to a government childcare programme to support healthy eating and physical activity offered to services. An audit of menus found intervention services were more likely to have fewer high-fat, salt or sugar processed meal items (intervention -0.9 items, control -0.2 items, $P=0.001$ ), fewer sweetened drinks (intervention -0.4 items, control -0.1 items, $P<0.001$ ) and more servings of vegetables (intervention +1.0 serves, control +0.2 serves, $P<0.001$ ) than control services.

Williams and colleagues conducted a nonrandomised trial of a childcare (preschool) education and food service intervention conducted in Head Start Centers in upstate New York (Bollella 1999; D'Agostino 1999; Spark 1998; Williams 1998; Williams 2002; Williams 2004). The primary aim was to reduce the saturated fat content of service meals and to reduce consumption of saturated fat by children. Six services received either a food service intervention with nutrition classroom education curricula or an identical food service intervention with a classroom safety component. Both of these groups received implementation support to improve food service. Three other childcare services with food operations not amenable to modification served as a control and received safety education curricula. Implementation of menus with nutrient content consistent with guideline recommendations was assessed by obtaining menu recipes and food labels over a five-day period. The study found within-group reductions in grams of saturated fat of food listed on menus, reducing from 11.3 grams (standard deviation $(S D) \pm 1.9)$ to 7.6 grams $(S D \pm 1.7)$ at the 18 -month follow-up $(P<0.05)$. Within-group changes were also identified for percentage of energy (kcal) from fat, reducing from $31.0(S D \pm 2.6)$ to $27.6(S D \pm 2.8)$ at six months $(P<0.05)$ and to $25.0(S D \pm 2.6)$ at 18 months $(P<0.01)$. Similarly, the percentage of energy (kcal) from saturated fat reduced from $12.5(S D \pm 1.4)$ to $10.3(S D \pm 1.4)$ at six months and to $8.0(\mathrm{SD} \pm 1.2)$ at the 18-month follow-up $(\mathrm{P}<$ $0.05)$ within the intervention group. There were no clear changes in these measures within the control group, or within either the intervention and control group for the other 15 nutrients measured at 18 month follow-up. Statistical comparisons between groups were not conducted. 


\section{Dichotomous outcomes}

Ten studies comparing an implementation strategy to usual care or no implementation support reported a dichotomous measure of implementation (Alkon 2014; Bell 2014; Finch 2012; Finch 2014; Finch 2019; Hardy 2010; Jones 2015; O'Neill 2017; Seward 2017; Stookey 2017). Pooling of data from the seven randomised trials reporting these outcomes (Alkon 2014; Finch 2014; Finch 2019; Hardy 2010; Jones 2015; Seward 2017; Stookey 2017) in meta-analysis found low-certainty evidence of an improvement in implementation favouring the group receiving implementation support in the proportion of services or staff implementing a policy or practice (OR $1.83 ; 95 \% \mathrm{Cl} 0.81$ to $4.11 ; \mathrm{I}^{2}=51 \% ; \mathrm{P}=0.14$; participants $=391$ services) (Analysis 1.2 ).

Sensitivity analysis was not performed as none of the randomised trials contributing to the meta-analysis was assessed as being at high risk of bias for these outcomes. As the 12 value was $51 \%$, subgroup analyses were not performed to investigate heterogeneity.

The three nonrandomised trials provided very low-certainty evidence regarding the effects of implementation strategies on per cent of services or staff implementing a policy or practice. Two Australian nonrandomised trials (Bell 2014; Finch 2012) examined the impact, relative to usual practice comparison of implementation strategies including educational materials, educational meetings, audit and feedback, opinion leaders and small incentives versus usual practice control. In the first nonrandomised trial by Finch and colleagues (Finch 2012), data collected via telephone interview revealed service managers in the intervention region were more likely to report a physical activity policy (intervention $+28 \%$, control $+4 \%, P<0.01$ ) with a physical activity policy that referred to limits on small screen recreation (intervention $+37 \%$, control $+5 \%, P<0.01$ ) and with staff trained in physical activity (intervention $+47 \%$, control $+6 \%, \mathrm{P}<$ $0.01)$. There were no clear differences between intervention and control services at follow-up in the proportion that conducted daily fundamental movement sessions with recommended components (intervention $+8 \%$, control $-1 \%, \mathrm{P}=0.08$ ); with a policy that referred to physical activity training for staff (intervention $+23 \%$, control $+8 \%, P=0.07$ ), where all staff usually participate in free active play (intervention $+7 \%$, control $+8 \%$ ), where all staff usually provide verbal prompts for physical activity (intervention $+2 \%$, control $+3 \%$ ), where children watch small screen recreation less than once per week (intervention $-1 \%$, control $-2 \%$ ), and where children participate in seated activities for no longer than 30 minutes at a time (intervention $+1 \%$, control $+3 \%)(P=0.65$ to 0.95$)$.

The second Australian nonrandomised trial by Bell and colleagues reported a number of improvements in implementation assessed using dichotomous measures (Bell 2014). Relative to the services in the control group, data from interviews with service managers found an increase in the proportion of services providing only water and plain milk to children (non-sweetened drinks). Within the intervention group, this increased from $68 \%$ at baseline to $95 \%$ at follow-up, compared with changes from $58 \%$ to $82 \%$ in control services $(P=0.02)$. The proportion of services where parents participated in nutrition programmes or policy development increased from $65 \%$ at baseline to $77 \%$ at follow-up for intervention services compared with a change from $65 \%$ to $59 \%$ in the control group $(P<0.01)$. There were no clear differences between groups in three other policies or practices examined and assessed via telephone interview with service managers. Furthermore, consistent with dietary guidelines, intervention services were more likely than control services to have no sweetened drinks listed on their menu (intervention $+46 \%$, control $+10 \%, \mathrm{P}<0.001$ ) and the appropriate servings of fruit (intervention $+34 \%$, control $+4 \%, \mathrm{P}$ $=<0.001$ ) and vegetables (intervention $+20 \%$, control $+4 \%, P=$ 0.01 ) listed on the menu. The differences between groups in service guideline adherence to recommendations regarding provision of high-fat, salt and sugar processed foods or water were small and uncertain (intervention effect sizes $+9 \%$ to $+10 \%, P=0.11$ to 1.00 ).

The final nonrandomised trial, undertaken in the USA, assessed the effects of an implementation strategy including educational meetings and guidelines, on the implementation of dietary and physical activity practices of services (O'Neill 2017). Implementation of these were assessed against sector standards using a tool based on the EPAO and incorporating observations and menu reviews undertaken at the service by trained data collectors. The study reported little difference across 13 dichotomous measures of implementation with odds ratios ranging from 1.35 ( $95 \% \mathrm{Cl}: 0.88$ to $1.44 ; \mathrm{P}=0.63$ ) to $0.89(95 \% \mathrm{Cl}: 0.75$ to $1.22 ; \mathrm{P}=0.09)$.

\section{Studies comparing alternative strategies to improve the implementation of any healthy eating, physical activity or obesity prevention policy, practice or programme in centre- based childcare services}

The two studies that compared the effects of two alternate implementation strategies reported mixed effects. One study used a continuous implementation outcome measure, and the other a dichotomous measure.

\section{Continuous outcomes}

Johnston Molloy and colleagues conducted a randomised (participants $=42$ services; low-certainty evidence), parallelgroup trial testing two training-based interventions to improve implementation of nutrition and health-related activity practices in Irish full daycare services (preschools) (Johnston Molloy 2013). Services were randomised to a 'manager and staff trained' group which received education materials, manager and staff educational meetings, and audit and feedback $(n=31)$ or a 'manager trained' only group receiving educational materials, manager (only) educational meetings, and audit and feedback $(n=30)$. Eighteen services in the 'manager and staff training' group and 24 in the 'manager trained' group provided follow-up data and were included in the main analysis. The total Preschool Health Promotion Activity Scored Evaluation did not differ between groups (absolute difference in median scores between 'manager and staff trained' versus 'manager trained' only group $=-2$ ), with median total scores improving from 15 to 34 in the 'manager and staff trained group' and 13 to 34 in the 'manager trained' only group $(\mathrm{P}=$ $0.84)$. Similarly, there were no clear between-group differences on any of four reported subscale measures of nutrition environment, food service, meals or snacks.

\section{Dichotomous outcomes}

Gosliner and colleagues conducted a randomised trial (participants $=13$ services; very low-certainty evidence) with staff from childcare services in the USA to assess the impact of an intervention on the nutrition and physical activity environment of childcare services (Gosliner 2010). Childcare services that were participating in a health education and policy development 
project (Child Health and Nutrition Center Enhancement) were matched on city of location and randomised to an intervention or control group. All services received multi-strategic implementation support including educational materials, educational meetings, educational outreach visits or academic detailing with small incentives or grants ('comparison'). Staff of intervention services additionally received a wellness programme consisting of individual health assessments (conducted by the research team); monthly newsletters and information with pay-checks promoting healthy eating and nutrition; a group walking programme where staff received collective incentive rewards as they reached milestones; and staff follow-up support visits.

At 10-month follow-up, a number of improvements on measures of implementation favouring the intervention group receiving the wellness programme were reported. Specifically, staff at intervention services were more likely to report providing fruit 'more often' to children in children's meals or snacks during the past year (74\% of staff) compared to staff at comparison services (41\% of staff) $(P=0.004)$. Similarly, staff at intervention services were more likely to report providing vegetables 'more often' to children in children's meals or snacks during the past year $(64 \%$ of staff) compared to staff at comparison services (38\% of staff) $(P=0.03)$. There were no clear differences between groups in the provision of sweetened beverages (intervention 7\%, control 8\%) and sweetened foods (intervention and comparison 5\%) ( $P$ values not reported). At children's celebrations during the past year, staff at intervention services were more likely to report providing fresh fruit (39\% of staff) compared to staff at control services $(24 \%$ of staff) $(P=0.05)$. Further, intervention staff reported providing fewer sweetened beverages ( $7 \%$ of staff) compared to comparison (27\% of staff) $(P=0.05)$ and fewer sweetened foods (intervention $15 \%$, control $34 \% ; P=0.025)$. There were no differences between groups in the provision of vegetables at children's celebrations (intervention 32\%, control 24\%; P value not reported).

\section{Subgroup analyses of strategies to improve implementation 'at scale'}

Three studies sought to implement policies or practices 'at scale', defined as more than 50 services (Bell 2014; Finch 2012; Ward 2008). The randomised trial of multiple strategies to implement the Nutrition and Physical Activity Self-Assessment in Child Care (NAPSACC) programme by Ward and colleagues was conducted in 56 intervention services and reported improvements in total EPAO score among services receiving implementation support (MD 1.01, $95 \% \mathrm{Cl} 0.18$ to 1.84 ) (Ward 2008).

A nonrandomised trial of implementation support provided to more than 200 childcare services reported improvement, favouring the intervention group, in the proportion of intervention services with a physical activity policy (percentage change in telephone interview measure: intervention $+28 \%$, control $+4 \%, P<0.01$ ) with a physical activity policy that referred to limits on small screen recreation (percentage change in telephone interview measure: intervention $+37 \%$, control $+5 \%, \mathrm{P}<0.01$ ) and with staff trained in physical activity (percentage change in telephone interview measure: intervention $+47 \%$, control $+6 \%, P<0.01$ ), but not on eight other measures (Finch 2012). Across all 11 practices, the median improvement of intervention relative to control was $2.5 \%$ (range $-4 \%$ to $41 \%)$.
Similarly, Bell and colleagues found, relative to the services in the control group, increases among services receiving implementation support in the proportion of services providing only water and plain milk to children (non-sweetened drinks) and a number of measures of the proportion of service menus with foods consistent with dietary guidelines (Bell 2014). Across 10 such measures, however, the median effect was $9.5 \%$ (range $2 \%$ to $36 \%$ ). An audit of menus revealed that intervention services had fewer high-fat, salt or sugar processed meal items (intervention - 0.9 items, control -0.2 items, $P$ $=0.001$ ), fewer sweetened drinks (intervention -0.4 items, control -0.1 items, $P<0.001$ ), and more servings of vegetables (intervention +1.0 serves, control +0.2 serves, $P<0.001$ ).

\section{Secondary outcomes}

\section{Estimates of absolute costs or assessments of cost-effectiveness}

None of the included studies reported on the costs or any cost analyses for the interventions.

\section{Reported adverse consequences}

Three studies explicitly assessed whether the intervention had unintended adverse effects (Finch 2014; Jones 2015; Seward 2017). Both the randomised trials by Finch and Jones found little difference in the number of child injuries in the month prior to assessment among intervention and comparison childcare services as reported by childcare managers at baseline and follow-up (Finch 2014; Jones 2015) (participants = 148 childcare services; two studies reporting continuous outcomes (child injury rates); low-certainty evidence). In the study by Finch and colleagues, the rate of injury per month at intervention services at baseline was 0.18 (95\% Cl 0.09 to 0.27$)$ and $0.17(95 \% \mathrm{Cl} 0.08$ to 0.27$)$ at follow-up, and at control services was $0.12(95 \% \mathrm{Cl} 0.04$ to 0.20$)$ at baseline and $0.11(95 \%$ $\mathrm{Cl} 0.03$ to 0.19$)$ at follow-up $(P=0.85)$ (Finch 2014). Similarly, in the trial by Jones and colleagues, the rate of serious child injuries at intervention services was $0.72(95 \% \mathrm{Cl} 0.39$ to 1.05$)$ and $0.90(95 \%$ $\mathrm{Cl} 0.52$ to 1.29$)$ at control services during the previous 12 months $(P=0.47)$ (Jones 2015). The rate of staff injuries was also assessed in the trial by Jones and there was little difference between groups with 0.77 (95\% $\mathrm{Cl} 0.49$ to 1.06 ) injuries on average at intervention services compared with $0.84(95 \% \mathrm{Cl} 0.42$ to 1.26$)$ at control services during the previous 12 months $(P=0.80)$ (Jones 2015).

In a randomised trial of a strategy to improve food services through implementation of nutrition guidelines in childcare, Seward and colleagues assessed negative feedback regarding the service menu from service educators, children and parents in the last month as reported by the service cook at follow-up (participants $=45$ childcare services; one study reporting dichotomous outcomes; very low-certainty evidence) (Seward 2017). There was no clear difference in negative feedback received from educators in intervention services $(7(32 \%))$ and control services $(4(25 \%))(P=$ $0.62)$; from children in intervention services $(7(32 \%))$ and control services $(1(6 \%))(P=0.07)$; and parents in intervention services $(2$ $(9 \%))$ and control services $(0(0 \%))(P=0.954)$.

\section{Effects on child diet, physical activity or weight status Diet}

Four of the 21 studies assessed the impact of the intervention on child dietary intake (Jones 2015; Seward 2017; Sharma 2018; Williams 2002). 
Of the two randomised trials (participants $=134$ children from 182 childcare services; two studies reporting continuous servebased measures of dietary intake; low-certainty evidence), one study used weighed food record methodology to assess the effectiveness of a multi-strategy implementation intervention on the aggregate servings of the core food groups and 'discretionary' foods consumed by children in care at baseline and follow-up (Seward 2017). Results from the RCT identified an improvement in consumption in the intervention services, relative to control, for vegetables (adjusted difference $=0.70 ; 95 \% \mathrm{Cl} 0.33$ to $1.08 ; \mathrm{P}<0.001$ ) and fruit (adjusted difference $=0.41 ; 95 \% \mathrm{Cl} 0.09$ to $0.73 ; \mathrm{P}=0.014$ ). Differences between groups in aggregate servings of discretionary serves (adjusted difference $=-0.54 ; 95 \% \mathrm{Cl}-0.14$ to $0.05, \mathrm{P}=0.073$ ) and dairy serves (adjusted difference $=-0.02,95 \% \mathrm{Cl}-0.48$ to 0.43 ), servings of breads and cereals (adjusted difference $=0.26,95 \% \mathrm{Cl}$ -0.67 to $1.21, \mathrm{P}=0.56$ ) and meat food groups (adjusted difference $=$ $0.13,95 \% \mathrm{Cl}-0.12$ to $0.38, \mathrm{P}=0.296$ ) were small and uncertain.

The second RCT by Jones and colleagues used direct observation to evaluate the effects of an intervention aimed at improving the implementation of healthy eating and physical activity policies on the mean number of serves consumed by children for each food group within the Australian Guide to Healthy Eating at followup (Jones 2015). Results at 12-month follow-up showed little difference between groups in the mean number of vegetable serves (intervention 0.1, SD 0.3; control 0.2, SD 0.6, $P=0.32$ ), fruit (intervention 1.1, SD 1.1; control 0.8, SD 0.7, $\mathrm{P}=0.14$ ), grain serves (intervention 1.6, SD 0.5; control 1.4, SD 0.8; $\mathrm{P}=0.28$ ) consumed by children in care. No differences were reported in the mean number of meat and alternatives (intervention 0.1, SD 0.2; control 0.1 SD 0.3; $P=0.67$ ), milk, yoghurt and cheese (intervention 0.7 SD 0.6; control 0.7 SD $0.7 ; P=0.97$ ) and discretionary food serves (intervention 0.7 SD 0.6; control 0.7, SD 0.7; $P=0.79$ ) consumed by children in care.

The nonrandomised trial by Williams and colleagues also used observational measures comparing child education curricula and a one-day food service modification training for cooks with a child curricula only control. Specifically, child dietary intake was assessed via direct observation during meal and snack periods (Williams 2002) (participants $=709$ children from nine services, one study, very low-certainty evidence). The intervention was primarily focused on reducing fat, saturated fat and energy. The study found that children attending intervention services consumed less energy $(-81.33 \mathrm{kcal})$, fat $(-3.6$ grams$)$, saturated fat $(-1.86$ grams $)$, as well as less fat as a percentage of energy (-4.48), and saturated fat as a percentage of energy $(-2.87)$ relative to the control at the six-month follow-up during attendance at care (all $P<0.001$ ). At the 18-month follow-up, the saturated fat (-2.56 grams) and fat as a percentage of energy (-10.92), and saturated fat as a percentage of energy (-5.15), remained lower relative to the control group $(P<0.001$ to 0.01$)$. The study also assessed changes in 13 other nutrients. Of these, intake of iron and magnesium were found to be higher among children in intervention compared with control services at the 18-month follow-up.

The remaining study, a nonrandomised trial (participants $=848$ children from 25 services, one study, very low-certainty evidence) with serial cross-sectional data collection, used parent self-report to assess the impact of a service-based nutrition and physical activity programme on changes in the frequency of child intake of various healthy and unhealthy foods at baseline and follow-up (Sharma 2018). Sharma and colleagues found little to no changes in frequency of fruit, $0.005(95 \% \mathrm{Cl}-0.13$ to $0.13, \mathrm{P}=0.940)$, vegetables, $-0.003(95 \% \mathrm{Cl}-0.14$ to $0.14, \mathrm{P}=0.996)$ and sports drink, $0.14(95 \% \mathrm{Cl}$ -0.002 to $0.29, P=0.054)$. Differences were found in the frequency of child intake of French fries, $0.21(95 \% \mathrm{Cl} 0.09$ to $0.33, \mathrm{P}=0.000)$ and sugar-sweetened beverages $-0.52(95 \% \mathrm{Cl}-0.70$ to $-0.35, \mathrm{P}=0.000)$.

\section{Physical activity}

Five studies assessed the impact of the intervention on child physical activity, providing little evidence of benefit (Alkon 2014; Finch 2014; Jones 2015; Mazzucca 2017; Sharma 2018), one of which was a nonrandomised trial (Sharma 2018).

Two of the five studies used objective methods, including childworn pedometers and accelerometers, to assess changes in child physical activity (participants $=420$ children from 46 services; two studies; high-certainty evidence). In the randomised trial of a multi-component intervention of 20 childcare services by Finch and colleagues, there was no improvement, relative to control, in the step counts per minute as assessed by pedometer for children attending intervention services (Finch 2014). Mean child step counts in the intervention group were $17.20(95 \% \mathrm{Cl} 15.94$ to 18.46) at baseline and 16.12 ( $95 \% \mathrm{Cl} 14.86$ to 17.30$)$ at follow-up, and in the control group step counts were $13.78(95 \% \mathrm{Cl} 12.76$ to $14.80)$ at baseline and $13.87(95 \% \mathrm{Cl} 12.57$ to 15.17$)$ at follow-up. Mazzucca and colleagues assessed difference between groups in total child physical activity, minutes per hour of being sedentary and different intensities of physical activity through accelerometers worn by children for five days at baseline and follow-up (Mazzucca 2017). Results of the trial indicated that children in the intervention arm averaged $480.2 \pm 9.3$ counts per minute at follow-up compared to $459.7 \pm 9.4$ counts per minute in the control group controlling for baseline $(P=0.12)$. Additionally, the trial reported small and uncertain difference $(P=0.13)$ in vigorous physical activity in children in the intervention group compared to those in the control, (5.6 versus $5.4 \mathrm{~min} / \mathrm{hr}$, respectively). No other differences were reported by authors in the amount of sedentary behaviour, total physical activity, or moderate to vigorous physical activity (MVPA).

Two studies used formal observational methods to assess changes in child physical activity (participants $=53$ childcare services; two studies; moderate-certainty evidence). In a randomised trial of a multi-component intervention to facilitate implementation of the NAPSACC programme, Alkon and colleagues found no significant changes in the intensity or type of physical activity of children in care as assessed by the Observation System for Recording Activity in Preschools (OSRAP) tool (effect sizes and $P$ value not reported) (Alkon 2014). There was, however, a nonsignificant change in the intervention group in the proportion of sedentary/quiet time, from $60 \%$ at baseline to $56 \%$ at follow-up, and a nonsignificant increase in the control group from $53 \%$ at baseline to $58 \%$ at follow-up ( $P$ value not reported). Jones and colleagues assessed differences between groups in the proportion of children engaged in sedentary, walking or very active physical activity during all observations, structured physical activity and outdoor free play sessions through researcher observation at follow-up (Jones 2015). Results of the randomised trial identified small and uncertain differences between groups in the proportion of children engaged in very active (intervention, $26.1 \%, 95 \% \mathrm{Cl} 22.5$ to 29.8 ; control, $21.3 \%, 95 \% \mathrm{Cl} 17.7$ to 24.9 ), walking (intervention, $29.1 \%$, $95 \%$ $\mathrm{Cl} 26.5$ to 31.7 ; control, $29.5 \%, 95 \% \mathrm{Cl} 27.2$ to 31.8 ) or sedentary (intervention, $44.8 \%, 95 \% \mathrm{Cl} 41.5$ to 48.1 ; control, $49.2 \%, 95 \% \mathrm{Cl}$ 45.8 to 52.5$)(\mathrm{P}=0.49)$ physical activity during all observations. 
Similarly for structured physical activity observations, small and uncertain differences between groups were observed in very active, (intervention, $40.3,95 \% \mathrm{Cl} 29.5$ to 51.0 ; control, 32.9, 95\% Cl 23.1 to 42.6 ), walking (intervention, $18.2,95 \% \mathrm{Cl} 10.4$ to 26.1 ; control, $25.7,95 \% \mathrm{Cl} 19.0$ to 32.5 ) or sedentary (intervention, $41.5,95 \% \mathrm{Cl}$ 31.1 to 51.9 ; control, $41.5,95 \% \mathrm{Cl} 31.3$ to 51.4$)(\mathrm{P}=0.64)$ physical activity. For outdoor free play observations, small and uncertain differences were observed between groups in the proportion of children engaged in very active (intervention, $22.2,95 \% \mathrm{Cl} 19.4$ to 25.1; control, $18.4,95 \% \mathrm{Cl} 15.3$ to 21.5 ), walking (intervention, 32.1, $95 \% \mathrm{Cl} 29.7$ to 34.5 ; control, $30.5,95 \% \mathrm{Cl} 27.9$ to 33.0 ) or sedentary physical activity (intervention, $45.7,95 \% \mathrm{Cl} 42.4$ to 49.0 ; control, $51.1,95 \% \mathrm{Cl} 48.1$ to 54.2$)(\mathrm{P}=0.47)$.

One nonrandomised trial (participants $=848$ children, one study, very low-certainty evidence) used parent self-report to compare the days children spent participating in more than 60 minutes of physical activity and the days playing outside for more than 30 minutes at baseline and follow-up (Sharma 2018). There was no difference between groups for mean number of days participating in more than 60 minutes of physical activity $(P=0.824)$. Similarly, the difference between groups for mean number of days spent playing outside for more than 30 minutes for children was unclear $(P=0.435)$.

\section{Weight status}

Five studies assessed the impact of the intervention on child weight status (Alkon 2014; Esquivel 2016; Sharma 2018; Stookey 2017; Williams 2002). All five studies objectively assessed child weight status through the collection of weight and height data by research staff or health workers during data collection, which was then used to calculate changes in mean BMI z-scores and BMI percentiles. Across these studies, the reported effects on BMI/ZBMI were mixed.

Of the five studies that assessed child weight status, three studies were RCTs (Alkon 2014; Esquivel 2016; Stookey 2017). Analyses of the impact of the intervention aiming to improve implementation of healthy eating and physical activity practices on centre-level child adiposity revealed a reduction in body mass index (BMI) $z$-score relative to the control group (coefficient -0.26 , standard error (SE) $0.1, P=0.02$ ) in the trial by Alkon and colleagues (Alkon 2014; low-certainty evidence). Two RCTs assessed changes in child weight status through mean changes in BMI percentiles and BMI zscores (Esquivel 2016; Stookey 2017; moderate-certainty evidence). Stookey and colleagues assessed annual mean changes in child BMI percentile and BMI z-score at baseline and follow-up periods after conducting an intervention to improve implementation of nutrition and physical activity practices (Stookey 2017). Mean BMI percentiles for children in the intervention group were 1.7 (SD 0.6) at baseline and -0.07 (SD 0.7) at follow-up, whilst BMI percentiles in the control group were 1.0 (SD 0.7) at baseline and -2.1 (SD 0.7 ) at two-year follow-up. Mean BMI z-scores in the intervention group decreased from 0.05 (SD 0.02) at baseline to -0.04 (SD 0.02), and in the control group decreased from 0 (SD 0.02) to -0.09 (SD 0.02 ) at two-year follow-up. The statistical significance of annual mean changes in BMI percentiles and z-score for both groups was not reported. Esquivel and colleagues assessed the impact of a childcare service policy intervention on mean child BMI z-scores (Esquivel 2016). Mean BMI z-scores increased for children in the intervention group from 0.51 (SD 1.14) at baseline to 0.60 (SD 1.16) at follow-up $(P=0.50)$, and in the control group increased from
0.25 (SD 1.14) at baseline to $0.35(\mathrm{SD} 1.17)$ at follow-up $(\mathrm{P}=0.48)$ following the seven-month intervention.

The remaining two studies that assessed child weight status were nonrandomised trials (Sharma 2018; Williams 2002; very lowcertainty evidence). Sharma and colleagues found lower mean child BMI z-scores $(-0.26,95 \% \mathrm{Cl}-0.50$ to $-0.01, \mathrm{P}=0.041)$ and mean $\mathrm{BMI}$ percentiles $(-6.5,95 \% \mathrm{Cl}-12.4$ to $-0.69, \mathrm{P}=0.028)$, in intervention services compared to control services following a twoyear study which focused on implementing a childcare servicebased nutrition and physical activity programme (Sharma 2018). An intervention focused on improving childcare menus by Williams and colleagues assessed change in child weight to height ratio at six-month follow-up. The study found no clear intervention effect (f-value 1.18, P value not reported) (Williams 2002).

\section{Implementation acceptability, adoption, penetration, sustainability and appropriateness}

\section{Acceptability}

Acceptability of implementation strategies was measured in eight of 21 included studies (Benjamin 2007 Finch 2012; Finch 2014; Finch 2019; Hardy 2010; Jones 2015; Mazzucca 2017; Ward 2017). All eight studies measured intervention acceptability utilising selfreport methods, including telephone interviews, surveys and focus groups conducted by implementation support staff with childcare service-nominated supervisors and staff.

Across studies, measures of the acceptability of educational materials by childcare staff (e.g. factsheets, newsletters, activity handbooks and policy templates), ranged from $60 \%$ to $100 \%$ (Benjamin 2007; Finch 2012; Finch 2014; Finch 2019; Jones 2015; Ward 2017), and educational outreach or academic detailing (e.g. training workshops) ranged from $88 \%$ to $100 \%$ (Benjamin 2007; Finch 2012; Finch 2014; Hardy 2010; Jones 2015). Five studies examined acceptability of the ongoing support provided throughout the intervention (Finch 2012; Finch 2019; Jones 2015; Mazzucca 2017; Ward 2017). Across studies, such support delivered via telephone was considered acceptable by $83 \%$ to $98 \%$ of childcare staff (Finch 2012; Finch 2019; Jones 2015; Mazzucca 2017; Ward 2017) while $98 \%$ to $100 \%$ reported such support via face-toface methods was acceptable (Jones 2015).

\section{Penetration}

Penetration of implementation strategies within intervention childcare services was examined in 12 of the 21 included studies (Alkon 2014; Finch 2012; Finch 2014; Hardy 2010; Gosliner 2010; Johnston Molloy 2013; Jones 2015; Mazzucca 2017; Seward 2017; Stookey 2017; Ward 2008; Yoong 2016). Of the 12 studies that measured penetration, three studies used self-report methods (Gosliner 2010; Stookey 2017; Yoong 2016), including interviews and surveys with childcare service staff, and five studies used internal records from implementation and research staff (Alkon 2014; Finch 2014; Jones 2015; Mazzucca 2017; Seward 2017). The remaining four studies (Finch 2012; Hardy 2010; Johnston Molloy 2013; Ward 2008) did not report how the penetration of intervention components was measured.

Across studies, measures of the penetration of educational materials (e.g. factsheets, newsletters, activity handbooks and policy templates) ranged from $37 \%$ to $100 \%$ (Finch 2012; Gosliner 2010; Jones 2015; Seward 2017; Stookey 2017; Yoong 2016). Eight 
studies examined penetration of educational outreach or academic detailing, ranging from $8 \%$ to $100 \%$ of childcare services (Alkon 2014; Finch 2012; Finch 2014; Gosliner 2010; Jones 2015; Johnston Molloy 2013; Seward 2017; Stookey 2017). Four studies measured penetration of ongoing support within the intervention (Finch 2012; Jones 2015; Mazzucca 2017; Seward 2017). Across these studies, the penetration of support delivered via telephone and email ranged from 69\% to 78\% (Finch 2012; Mazzucca 2017), and penetration of face-to-face support ranged from $76 \%$ to $96 \%$ (Jones 2015; Seward 2017).

\section{Adoption}

None of the included studies reported on the adoption of the interventions.

\section{Sustainability}

None of the included studies reported on the sustainability of the interventions.

\section{Appropriateness}

None of the included studies reported on the appropriateness of the interventions.

\section{DISCUSSION}

\section{Summary of main results}

This review sought to assess the impact of strategies to support the implementation of policies, practices or programmes to promote physical activity, healthy eating or prevent excessive weight gain among children in centre-based childcare services. The review identified 21 studies, most of which were randomised controlled trials testing multi-component implementation support strategies. Collectively, the findings suggest that implementation strategies are likely to improve the implementation of policies, practices or programmes that promote child healthy eating, physical activity and/ or obesity prevention in childcare services. Meta-analysis of randomised trials reporting score-based measures of implementation (e.g. physical activity environment and policy assessment observation) found effects favouring implementation support strategies on these outcomes. Metaanalysis of randomised trials reporting dichotomous outcomes (e.g. proportion of services implementing a policy or practice), reported an $80 \%$ increase in the odds of implementation favouring childcare services that received implementation support. While this effect is uncertain as the $95 \%$ confidence intervals are inclusive of values of no effect, the point estimate was relatively large. There was little evidence that interventions, and the strategies employed to implement them improved child diet, physical activity or weight status. No studies reported cost or cost-effectiveness outcomes.

There were a number of challenges in conducting and synthesising the findings of included studies. Classification of implementation strategies was difficult. The Cochrane Effective Practice and Organisation of Care (EPOC) Group taxonomy has been developed to describe strategies to improve implementation or professional practice of health services or practitioners, which were often not relevant for the childcare setting (EPOC 2015). Other strategies employed by included studies to facilitate implementation, including small incentives such as lotteries or wellness initiatives, did not fit with the current EPOC taxonomy descriptors. To address such issues, we included full descriptions of studies, study context and implementation strategies. Despite the existence of other taxonomies that have been developed to consider community based interventions (Powell 2015), a revision of the EPOC taxonomy and descriptors to align more with the implementation strategies used in non-clinical settings may improve EPOC strategy coverage and facilitate classification for studies undertaken in childcare and other community settings. Interpretation of the findings therefore represents a challenge.

The lack of effectiveness of reported on measures of child diet, physical activity or weight status is concerning. There may be a number of possible explanations for the equivocal impacts found in this review on these outcomes. First, the interventions implemented in the included studies may not be effective in improving child health behaviours. In many studies, prior evidence supporting the efficacy of the intervention being implemented was not reported or was unclear (Johnston Molloy 2013; Sharma 2018; Stookey 2017). Ineffective interventions cannot improve child health outcomes, regardless of how well they are implemented in childcare services. Second, the efficacy of interventions in childcare services are often established in ideal research conditions. Even in circumstances when there is strong evidence supporting the efficacy of interventions, systematic reviews suggest intervention effect sizes typically attenuate when evaluated in more real world contexts (Finch 2015; McCrabb 2019; Yoong 2014b) due to a range of study, intervention and contextual factors. As implementation studies, by nature, are undertaken in more naturalistic environments, the effects of interventions may be reduced to the point that they no longer provide therapeutic benefit.

Finally, the findings may suggest the level of implementation achieved was insufficient to accrue improvement on such child health outcomes. If this is the case, more effective implementation approaches are required. Further, enhancing implementation, however, may represent a challenge. Childcare services report a broad range of factors that impede implementation including a lack of support from childcare executive committees, the service manager or parents (Wolfenden 2015a), staff members' own healthy eating or physical activity behaviours, self-efficacy in facilitating healthy eating or physical activity of children, and negative staff attitudes (Cashmore 2008; Copeland 2011; Froehlich Chow 2011). Furthermore, for the implementation of physical activity policies, practices and programmes in particular, structural barriers, such as a preference for child-directed rather than teacher-led structured physical activity by childcare service staff, a lack of space, inclement weather or lack of broader policy framework (Cashmore 2008; Copeland 2011), have been noted as implementation barriers. The selection of 'simple' interventions that may be more amenable to implementation, or interventions with larger effects may improve the likelihood that interventions, and strategies to implement them produce meaningful health outcomes for children.

\section{Overall completeness and applicability of evidence}

Twelve of the 21 included studies were conducted by two research groups in the USA and Australia (Alkon 2014; Bell 2014; Benjamin 2007; Finch 2012; Finch 2014; Finch 2019; Jones 2015; Mazzucca 2017; Seward 2017; Ward 2008; Ward 2017; Yoong 2016). Furthermore, all of the included studies were conducted in high-income countries. The applicability of study findings 
to lower and middle-income countries, where the operational, philosophical and cultural contexts may differ substantially, is unknown (Rosemburg 2003). Future research, conducted by a greater range of research groups in different research contexts, would strengthen the applicability of the evidence base.

\section{Quality of the evidence}

GRADE assessments varied by outcomes reported in the review, but were typically low. Risk of performance bias (due to lack of blinding of participants or personnel), detection bias (due to use of selfassessment measures in some studies) and reporting bias (due to a lack of prospective registration or published study protocols) were particularly prevalent among included studies. The comparison groups used limited the directness of the assembled evidence. A number of studies included comparison groups that included some active implementation support (Gosliner 2010; Johnston Molloy 2013), or 'usual' implementation support (Bell 2014; Finch 2012; Jones 2015; Seward 2017; Stookey 2017; Yoong 2016), which may not have been well defined. Finally, there were concerns regarding the precision of the estimates of included studies for the primary outcomes of this review. Most studies included samples of fewer than 15 per study arm, which is likely to be insufficient to detect small but meaningful effects. Similarly, 13 of the 21 studies included a measure of implementation as the primary study outcome (Alkon 2014; Bell 2014; Benjamin 2007; Esquivel 2016; Finch 2012; Gosliner 2010; Johnston Molloy 2013; Jones 2015; O'Neill 2017; Seward 2017; Ward 2008; Ward 2017; Yoong 2016), and only seven of these performed a sample size calculation to justify the included sample (Finch 2012; Finch 2019; Jones 2015; O'Neill 2017; Seward 2017; Ward 2017; Yoong 2016).

\section{Potential biases in the review process}

The review included a comprehensive search strategy for peerreviewed and grey literature and examined over 11,000 citations. We also sought relevant studies from screening of citations of included studies, and from contact with experts in the field. While the search strategy was rigorous, as this is a field in which terminology for implementation constructs are developing, it is possible that not all studies that report implementation outcomes were identified. For example, it has been estimated that $15 \%$ of studies use implementation strategies that cannot be classified using implementation taxonomies (Mazza 2013). Potentially relevant studies may have been missed based on the implementation strategy search terms used in this review. However, a previous review conducted by the Agency for Healthcare Research and Quality failed to identify any studies of implementation strategies targeting healthy eating and physical activity in the childcare setting (Rabin 2010), and contact with other experts in the field did not yield any additional studies to those identified in the primary search. Such findings provide some evidence to suggest that the search strategy may have provided reasonable coverage of the relevant literature. Nonetheless, we will assess the appropriateness of search terms in future updates of the review to ensure that the search terms are inclusive of relevant implementation terminology and newly released taxonomies. The method for describing effects across studies may have also introduced bias. In instances where a primary implementation outcome was not identified in included studies, we utilised a median effect size across implementation outcomes. Such analyses are inconsiderate of the robustness of individual measures, and may mask important effects on single implementation outcomes.
Consideration of the narrative description of each study included in the review is therefore important when interpreting study findings.

\section{Agreements and disagreements with other studies or reviews}

Similarly to findings of this review, other recent systematic reviews examining the effectiveness of implementation strategies in community settings, including workplaces (Wolfenden 2018), schools (Wolfenden 2017) and sporting clubs (McFadyen 2018), have reported a relatively small evidence base, and limited reporting of cost and cost-effective analyses. The findings of this review, however, provide more certainty regarding the effectiveness of strategies to improve implementation of health promotion polices and practices in this setting, compared to reviews of studies in other community organisations. Consistent with systematic reviews of implementation strategies in clinical settings, the findings of this review suggest that multi-strategic approaches can be effective in improving implementation (Squires 2014). Unlike reviews of health care, however (Forsetlund 2009; Ivers 2012; O'Brien 2007), the limited number of studies and heterogeneity of strategies used did not enable isolation of the effects of individual implementation strategies, or specific combinations thereof.

\section{AUTHORS' CONCLUSIONS}

\section{Implications for practice}

The review highlights the limited evidence base to guide policy makers and practitioners interested in supporting the implementation of healthy eating, physical activity or obesity prevention policies, practices and programmes in centrebased childcare services. Collectively, the findings suggest that implementation strategies can have a positive impact on the implementation. With a small number of studies to date and in the absence of high-quality evidence, formative work to achieve a comprehensive understanding of the setting, context and barriers to implementation, and careful selection of support strategies to address these, may be particularly important for practitioners to maximise the potential for successful implementation (French 2012).

\section{Implications for research}

The findings of this review suggest that there is considerable scope to improve the evidence base to guide future efforts to support implementation of healthy eating, physical activity and obesity prevention programmes in centre-based childcare services. The limited number of studies is surprising given the large numbers of studies testing interventions to improve healthy eating, physical activity or obesity prevention interventions in recent systematic reviews in this setting (Finch 2016; Sisson 2016; Stacey 2017). The findings confirm bibliographic studies that indicate that studies examining the effects of strategies to implement evidence-based programmes or polices represent a fraction of public health research studies (Wolfenden 2016a; Wolfenden 2016c; Yoong 2015). Greater investment in research, and research infrastructure to support studies to improve dissemination and implementation of effective childcare-based interventions, is therefore warranted (Wolfenden 2016b). Additionally, the review identified a number of ongoing studies in the area, which will further contribute to the evidence base (see Characteristics of ongoing studies). 
In many instances, the studies included in the review had small samples (Alkon 2014; Benjamin 2007; Esquivel 2016; Finch 2014; Gosliner 2010; Hardy 2010; Morshed 2016; Sharma 2018; Williams 2002), which may be unable to detect important improvements in policy or practice, a commonly faced challenge reported in the development of the implementation science literature, or they used self-reported measures of implementation. The cost of practice improvements was not assessed in any included studies and only nine studies assessed the impact of interventions on child health behaviours or weight status (Alkon 2014; Esquivel 2016 Finch 2014; Jones 2015; Mazzucca 2017; Seward 2017; Sharma 2018; Stookey 2017; Williams 2002). Comprehensive evaluations of future efforts to improve the implementation of health-promoting initiatives targeting excessive weight gain or its determinants in this setting are required to address the limitations identified within the existing evidence base. The use of hybrid designs in future studies, in which implementation outcomes as well as impacts on health behaviours or weight status have been recommended, is one means of achieving this (Cohen 2015).

Half of the included studies developed implementation support strategies without the aid of relevant theory or theoretical frameworks (Alkon 2014; Finch 2012; Gosliner 2010; Hardy 2010; Johnston Molloy 2013; O'Neill 2017; Stookey 2017; Ward 2017; Williams 2002). Perhaps unsurprisingly, the use of the range of potential strategies, as described in the EPOC taxonomy, was relatively limited by the included studies, and focused often on one-off training or resource provision. The factors that influence policy or practice implementation are typically complex. Improvements in implementation may require ongoing changes to systems and processes rather than fixed discrete support. However, none of the studies included strategies to address other fiscal, political, regulatory or governance factors that could potentially influence the success of implementation efforts. The use of comprehensive theoretical frameworks could assist in considering a broad range of implementation barriers and designing appropriate support strategies to address these (Cane 2012; Damschroder 2009). Further, future theoretically informed research to identify the mechanism by which support strategies may facilitate implementation would be of particular value to guide future strategy design (Lee 2018).

\section{ACKNOWLEDGEMENTS}

We would like to acknowledge the support provided by the University of Newcastle, Hunter New England Population Health, the Hunter Medical Research Institute and Cochrane Public Health. We would also like to acknowledge contributions made to the original review by Vivian Welch, Amanda J Williams, Benjamin Parmenter, Kirsty Seward, Tameka McFayden, Christopher Williams and John Wiggers. 


\section{RE F E R E N C E S}

\section{References to studies included in this review}

\section{Alkon 2014 \{published data only\}}

Alkon A, Crowley AA, Benjamin Neelon SE, Hill S, Pan Y, Nguyen $\mathrm{V}$, et al. Nutrition and physical activity randomized control trial in child care centers improves knowledge, policies, and children's body mass index. BMC Public Health 2014;14(215):1-13. [DOI: 10.1186/1471-2458-14-215]

\section{Bell 2014 \{published data only\}}

Bell AC, Davies L, Finch M, Wolfenden L, Francis JL, Sutherland R, et al. An implementation intervention to encourage healthy eating in centre-based child-care services: impact of the Good for Kids Good for Life programme. Public Health Nutrition 2014;18(9):1610-9. [DOI: 10.1017/ S1368980013003364]

\section{Benjamin 2007 \{published data only\}}

Benjamin SE, Ammerman A, Sommers J, Dodds J, Neelon B, Ward DS. Nutrition and physical activity self-assessment for child care (NAP SACC): results from a pilot intervention. Journal of Nutrition Education and Behavior 2007;39(3):142-9. [DOI: 10.1016/j.jneb.2006.08.027]

\section{Esquivel 2016 \{published data only\}}

Esquivel MK, Nigg CR, Fialkowski MK, Braun KL, Li F, Novotny R. Influence of teachers' personal health behaviors on operationalizing obesity prevention policy in Head Start Preschools: a project of the Children's Healthy Living Program (CHL). Journal of Nutrition Education and Behavior 2016;48(5):318-25.

\section{Finch 2012 \{published data only\}}

Finch M, Wolfenden L, Falkiner M, Edenden D, Pond N, Hardy LL, et al. Impact of a population based intervention to increase the adoption of multiple physical activity practices in centre based childcare services: a quasi experimental, effectiveness study. International Journal of Behavioral Nutrition and Physical Activity 2012;9(101):1-13. [DOI: doi:10.1186/1479-5868-9-101]

\section{Finch 2014 \{published data only\}}

Finch M, Wolfenden L, Morgan PJ, Freund M, Jones J, Wiggers J. A cluster randomized trial of a multi-level intervention, delivered by service staff, to increase physical activity of children attending center-based childcare. Preventive Medicine 2014;58:9-16. [DOI: 10.1016/j.ypmed.2013.10.004]

\section{Finch 2019 \{published data only\}}

Finch M, Stacey F, Jones J, Yoong S.L, Grady A, Wolfenden L. $A$ randomised controlled trial of performance review and facilitated feedback to increase implementation of healthy eating and physical activity-promoting policies and practices in centre-based childcare. Implementation Science 2019;14(1):17.

\section{Gosliner 2010 \{published data only\}}

Gosliner WA, James P, Yancey AK, Ritchie L, Studer N, Crawford PB. Impact of a worksite wellness program on the nutrition and physical activity environment of child care centers. American Journal of Health Promotion 2010;24(3):186-9. [DOI: 10.4278/ajhp.080227l9]

\section{Hardy 2010 \{published data only\}}

Hardy LL, King L, Kelly B, Farrell L, Howlett S. Munch and Move: evaluation of a preschool healthy eating and movement skill program. International Journal of Behavioral Nutrition and Physical Activity 2010;7(80):1-11. [DOI: doi:10.1186/1479-5868-7-80]

\section{Johnston Molloy 2013 \{published data only\}}

Johnston Molloy C, Kearney J, Hayes N, Glennon Slattery C, Corish C. Pre-school manager training: a cost-effective tool to promote nutrition- and health-related practice improvements in the Irish full-day-care pre-school setting. Public Health Nutrition 2013;18(9):1554-64. [DOI: 10.1017/S1368980013002760]

\section{Jones 2015 \{published and unpublished data\}}

Jones J, Wyse R, Finch M, Lecathelinais C, Wiggers J, Marshall J, et al. Effectiveness of an intervention to facilitate the implementation of healthy eating and physical activity policies and practices in childcare services: a randomised controlled trial. Implementation Science 2015;10:147.

\section{Mazzucca 2017 \{published and unpublished data\}}

Mazzucca SL. Physical Activity and Sedentary Behavior in Early Care and Education Centers: Identifying Opportunities and Testing Strategies to Support Active Classroom Environments. Chapel Hill, 2017.

\section{Morshed 2016 \{published data only\}}

Morshed AB, Davis SM, Keane PC, Myers OB, Mishra SI. The Impact of the CHILE intervention on the food served in Head Start centers in rural New Mexico. Journal of School Health 2016;86(6):414-23.

O'Neill 2017 \{published data only (unpublished sought but not used)\}

Neelon SEB, Mayhew M, O'Neill JR, Neelon B, Li F, Pate RR. Comparative evaluation of a South Carolina policy to Improve nutrition in child care. Journal of the Academy of Nutrition and Dietetics 2016;116(6):949-56.

* O'Neill JR, Dowda M, Neelon SEB, Neelon B, Pate RR. Effects of a new state policy on physical activity practices in child care centers in South Carolina. American Journal of Public Health 2017;107(1):144-6.

\section{Seward 2017 \{published data only\}}

Seward K, Wolfenden L, Finch M, Wiggers J, Wyse R, Jones J, et al. Improving the implementation of nutrition guidelines in childcare centres improves child dietary intake: findings of a randomised trial of an implementation intervention. Public Health Nutrition 2017;21:1-11.

\section{Sharma 2018 \{published data only\}}

Sharma S, Vandewater E, Chuang R, Byrd-Williams C, Kelder C, Butte N, et al. Impact of the coordinated approach to child health early childhood program for obesity prevention among 
preschool children: the Texas Childhood Obesity Research Demonstration Study. Childhood Obesity 2019;15(1):1-13.

\section{Stookey 2017 \{published data only\}}

Stookey JD, Evans J, Chan C, Tao-Lew L, Arana T, Arthur S. Healthy apple program to support child care centers to alter nutrition and physical activity practices and improve child weight: a cluster randomized trial. BMC Public Health 2017;17(1):965.

\section{Ward 2008 \{published data only\}}

Ward DS, Benjamin SE, Ammerman AS, Ball SC, Neelon BH, Bangdiwala SI. Nutrition and physical activity in child care. Results from an environmental intervention. American Journal of Preventive Medicine 2008;35(4):352-6. [DOI: 10.1016/ j.amepre.2008.06.030]

\section{Ward 2017 \{published data only\}}

Ward DS, Vaughn AE, Mazzucca S, Burney R. Translating a child care based intervention for online delivery: development and randomized pilot study of Go NAPSACC. BMC Public Health 2017;17(1):891.

\section{Williams 2002 \{published data only\}}

Williams CL, Bollela MC, Strobino BA, Spark A, Nicklas TA, Tolosi LB, et al. "Healthy-Start": outcome of an intervention to promote a heart healthy diet in preschool children. Journal of the American College of Nutrition 2002;21(1):62-71. [DOI: 10.1080/07315724.2002.10719195]

\section{Yoong 2016 \{published data only\}}

Yoong SL, Jones J, Marshall J, Wiggers J, Seward K, Finch M, et al. A theory-based evaluation of a dissemination intervention to improve childcare cooks' intentions to implement nutritional guidelines on their menus. Implementation Science 2016;11(1):105.

\section{References to studies excluded from this review}

\section{Adamo 2015 \{published data only\}}

Adamo K, Wilson S, Harvey A, Grattan K, Naylor P, Temple V, et al. Does intervening in childcare settings impact fundamental movement skill development?. Medicine \& Science in Sports \& Exercise 2015;48(1):42-9.

\section{Adamo 2017 \{published data only\}}

Adamo KB, Wasenius NS, Grattan KP, Harvey ALJ, Naylor P-J, Barrowman NJ, et al. Effects of a preschool intervention on physical activity and body composition. Journal of Pediatrics 2017;188:42-9.

\section{Bardid 2017 \{published data only\}}

Bardid F, Lenoir M, Huyben F, De Martelaer K, Seghers J, Goodway JD, et al. The effectiveness of a community-based fundamental motor skill intervention in children aged 3-8 years: results of the "Multimove for Kids" project. Journal of Science and Medicine in Sport 2017;20(2):184-9.

\section{Bell 2015 \{published data only\}}

Bell LK, Hendrie GA, Hartley J, Golley RK. Impact of a nutrition award scheme on the food and nutrient intakes of 2- to 4- year-olds attending long day care. Public Health Nutrition 2015;18(14):2634-42.

\section{Birnbaum 2017 \{published data only\}}

Birnbaum J, Geyer C, Kirchberg F, Manios Y, Koletzko B. Effects of a kindergarten-based, family-involved intervention on motor performance ability in 3- to 6-year-old children: the ToyBoxstudy. Journal of Sports Sciences 2017;35(4):377-84.

\section{Brand 2017 \{published data only\}}

Brand T, Jahn I, Pohlabeln H, Böttcher S, Hense S, Antje H, et al. Comparing strategies to improve the implementation of healthy nutrition in kindergartens: a prospective observational study. Journal of Public Health 2016;25(3):299-310.

\section{Brian 2017a \{published data only\}}

Brian A, Goodway J, Logan J, Sutherland S. SKIPing with Head Start teachers: influence of T-SKIP on object-control skills. Research Quarterly for Exercise and Sport 2017;88(4):479-91.

\section{Brian 2017b \{published data only\}}

Brian A, Taunton S. Effectiveness of motor skill intervention varies based on implementation strategy. Physical Education and Sport Pedagogy 2017;23(2):222-33.

\section{Burkart 2018 \{published data only\}}

Sarah B, Jasmin R, Matthew CD, Sofiya A. Behavioral effects of a locomotor-based physical activity intervention in preschoolers. Journal of Physical Activity and Health 2018;15(1):46-52.

\section{Byun 2018 \{published data only\}}

Byun W, Lau EY, Brusseau TA. Feasibility and effectiveness of a wearable technology-based physical activity intervention in preschoolers: a pilot study. International Journal of Environmental Research and Public Health 2018;15(9):E1821.

\section{Chuang 2018 \{published data only\}}

Chuang R-J, Sharma SV, Perry C, Diamond P. Does the CATCH early childhood program increase physical activity among lowincome preschoolers? - results from a pilot study. American Journal of Health Promotion 2018;32(2):344-8.

\section{Davis 2016 \{published data only\}}

Davis SM, Myers OB, Cruz TH, Morshed AB, Canaca GF, Keane PC, et al. CHILE: outcomes of a group randomized controlled trial of an intervention to prevent obesity in preschool Hispanic and American Indian children. Preventive Medicine 2016;89:162-8.

\section{De Craemer 2017 \{published data only\}}

De Craemer M, Verloigne M, De Bourdeaudhuij I, Androutsos O, lotova V, Moreno L, et al. Effect and process evaluation of a kindergarten-based, family-involved cluster randomised controlled trial in six European countries on four- to six-year-old children's steps per day: the ToyBox-study. International Journal of Behavioral Nutrition and Physical Activity 2017;14(1):116.

\section{Driediger 2018 \{published data only\}}

Driediger M, Vanderloo LM, Burke SM, Irwin JD, Gaston A, Timmons BW, et al. The implementation and feasibility of the supporting physical activity in the childcare environment

Strategies to improve the implementation of healthy eating, physical activity and obesity prevention policies, practices or programmes 
(SPACE) intervention: a process evaluation. Health Education \& Behavior 2018;45(6):935-44.

\section{Foulkes 2017 \{published data only\}}

Foulkes JD, Knowles Z, Fairclough SJ, Stratton G, O’Dwyer M, Ridgers ND, et al. Effect of a 6-week active play intervention on fundamental movement skill competence of preschool children: a cluster randomized controlled trial. Perceptual and Motor Skills 2017;124(2):393-412.

\section{Gelli 2017 \{published data only\}}

Gelli A, Roschnik N. A cluster randomised control trial of an integrated agriculture-nutrition package to improve children's diets through community based childcare centres in Malawi. Annals of Nutrition and Metabolism 2017;71(2):685-6.

\section{Goldfield 2016 \{published data only\}}

Goldfield GS, Harvey ALJ, Grattan KP, Temple V, Naylor P-J, Alberga AS, et al. Effects of child care intervention on physical activity and body composition. American Journal of Preventive Medicine 2016;51(2):225-31.

\section{Hu 2017 \{published data only\}}

Hu Y, He J-R, Liu F-H, Li W-D, Lu J-H, Xing Y-F, et al. Effectiveness of a kindergarten-based intervention for preventing childhood obesity. Pediatrics 2017;140(6):e20171221.

\section{ISRCTN94022291 \{published data only\}}

ISRCTN94022291. Activity Begins in Childhood (ABC) - inspiring healthy active behaviour in preschoolers: study protocol for a cluster randomized controlled trial. apps.who.int/ trialsearch/Trial2.aspx?TrialID=ISRCTN94022291 (first received 21 December 2012).

\section{Jones 2016 \{published data only\}}

Jones RA, Okely AD, Hinkley T, Batterham M, Burke C. Promoting gross motor skills and physical activity in childcare: a translational randomized controlled trial. Journal of Science and Medicine in Sport 2016;19(9):744-9.

\section{Lau 2017 \{published data only\}}

Lau EY, Saunders RP, Beets MW, Cai B, Pate RR. Factors influencing implementation of a preschool-based physical activity intervention. Health Education Research 2017;32(1):69-80.

\section{Lumeng 2017 \{published data only\}}

Lumeng JC, Miller AL, Horodynski MA, Brophy-Herb HE, Contreras $\mathrm{D}$, Lee $\mathrm{H}$, et al. Improving self-regulation for obesity prevention in Head Start: a randomized controlled trial. Pediatrics 2017;139(5):1-10.

\section{Malden 2018 \{published data only\}}

Malden S, Hughes AR, Gibson A-M, Bardid F, Androutsos O, De Craemer M, et al. Adapting the ToyBox obesity prevention intervention for use in Scottish preschools: protocol for a feasibility cluster randomised controlled trial. BMJ Open 2018;8(10):e023707.

\section{Mattingly 2016 \{published data only\}}

Mattingly JA, Andresen PA. NAP SACC: implementation of an obesity prevention intervention in an American Indian Head Start program. Journal of Community Health Nursing 2016;33(3):145-53.

\section{McSweeney 2017 \{published data only\}}

McSweeney L, Araújo-Soares V, Rapley T, Adamson A. A feasibility study with process evaluation of a preschool intervention to improve child and family lifestyle behaviours. BMC Public Health 2017;17(1):248.

\section{Natale 2017 \{published data only\}}

Natale RA, Messiah SE, Asfour LS, Uhlhorn SB, Englebert NE, Arheart KL. Obesity prevention program in childcare centers: two-year follow-up. American Journal of Health Promotion 2016;31(6):502-10.

\section{NCT02789215 \{published data only\}}

NCT02789215. Proposed meal changes for CACFP: impact on child food intake and costs. clinicaltrials.gov/ct2/show/ nct02789215 (first received 2 June 2016).

NCT03022472 \{published data only\}

NCT03022474. Kindergarten-based intervention for childhood obesity in Guangzhou (KICOG). clinicaltrials.gov/show/ nct03022474 (first received 2017).

\section{NCT03713840 \{published data only\}}

NCT03713840. Community partnering to encourage healthy beverage intake through child care. clinicaltrials.gov/ct2/show/ NCT03713840 (first received 22 October 2018).

\section{Nezami 2018 \{published data only\}}

Nezami BT, Ward DS, Lytle LA, Ennett ST, Tate DF. A mHealth randomized controlled trial to reduce sugar-sweetened beverage intake in preschool-aged children. Pediatric Obesity 2018;13(11):668-76.

\section{Pate 2016 \{published data only\}}

Pate RR, Brown WH, Pfeiffer KA, Howie EK, Saunders RP, Addy $\mathrm{CL}$, et al. An intervention to increase physical activity in children: a randomized controlled trial with 4-year-olds in preschools. American Journal of Preventive Medicine 2016;51(1):12-22.

\section{Pinket 2016 \{published data only\}}

Pinket A-S, Van Lippevelde W, De Bourdeaudhuij I, Deforche B, Cardon G, Androutsos O, et al. Effect and process evaluation of a cluster randomized control trial on water intake and beverage consumption in preschoolers from six European countries: the ToyBox-study. PLOS ONE 2016;11(4):e0152928.

\section{Razak 2018 \{published data only\}}

Razak LA, Yoong SL, Wiggers J, Morgan PJ, Jones J, Finch M, et al. Impact of scheduling multiple outdoor free-play periods in childcare on child moderate-to-vigorous physical activity: a cluster randomised trial. International Journal of Behavioral Nutrition and Physical Activity 2018;15(1):34. 
Roth 2015 \{published data only\}

Roth K, Kriemler S, Lehmacher W, Ruf K, Graf C, Hebestreit H. Effects of a physical activity intervention in preschool children. Medicine and Science in Sports and Exercise 2015;47(12):2542-51.

\section{Truelove 2016 \{published data only\}}

Truelove S, Tucker P. Change in preschoolers' health-related quality of life following the implementation of a childcare physical activity intervention. Pediatric Exercise Science 2016;28:36.

\section{Truelove 2018 \{published data only\}}

Truelove S, Johnson AM, Vanderloo LM, Driediger M, Burke SM, Irwin JD, et al. Preschoolers' health-related quality of life following the implementation of a childcare physical activity intervention. Applied Physiology, Nutrition, and Metabolism 2017;43(5):453-9.

\section{Tucker 2016 \{published data only\}}

Tucker P, Burke SM, Gaston A, Irwin JD, Johnson AM, Timmons BW, et al. Supporting physical activity in the childcare environment (SPACE): rationale and study protocol for a cluster randomized controlled trial. BMC Public Health 2016;16(1):112.

\section{Tucker 2017 \{published data only\}}

Tucker P, Vanderloo LM, Johnson AM, Burke SM, Irwin JD, Gaston A, et al. Impact of the Supporting Physical Activity in the Childcare Environment (SPACE) intervention on preschoolers' physical activity levels and sedentary time: a single-blind cluster randomized controlled trial. International Journal of Behavioral Nutrition and Physical Activity 2017;14(1):120.

\section{Vanderloo 2016 \{published data only\}}

Vanderloo LM, Tucker P, Gaston A, Timmons BW, Johnson AM, Burke SM, et al. Supporting Physical Activity in the Childcare Environment (SPACE): a cluster randomized controlled trial. Pediatric Exercise Science 2016;28:27.

\section{References to ongoing studies}

Helland 2016 \{published data only\}

Helland SH, Bere E, Overby NC. Study protocol for a multicomponent kindergarten-based intervention to promote healthy diets in toddlers: a cluster randomized trial. BMC Public Health 2016:16:273.

\section{Hennink-Kaminskia 2017 \{published data only\}}

Hennink-Kaminski H, Vaughn AE, Hales D, Moore RH, Luecking CT, Ward DS. Parent and child care provider partnerships: protocol for the Healthy Me, Healthy We (HMHW) cluster randomized control trial. Contemporary Clinical Trials 2017; Vol. 64:49-57.

\section{Kobel 2017 \{published data only\}}

Kobel S, Wartha O, Wirt T, Dreyhaupt J, Lammle C, Friedemann EM, et al. Design, implementation, and study protocol of a kindergarten-based health promotion intervention. BioMed Research International 2017;2017:4347675.

\section{Messiah 2016 \{published data only\}}

Messiah SE, Lebron C, Moise R, Sunil MM, Sardinas K, Chang C, et al. Healthy caregivers - healthy children ( $\mathrm{HC2}$ ) phase 2: integrating culturally sensitive childhood obesity prevention strategies into childcare center policies. Contemporary Clinical Trials 2017;53:60-7.

NCT01890681 \{published data only\}

NCT01890681. Baby nutrition and physical activity self-assessment for child care (NAP SACC) intervention study. clinicaltrials.gov/ct2/show/NCT01890681? term=childcare\&rank=15 (first received 2 July 2013).

NCT02375490 \{published data only\}

NCT02375490. Healthy start to increase physical activity and improve healthy eating in early childcare centres. clinicaltrials.gov/show/NCT02375490/ (first received 2 March 2015).

\section{NCT03075085 \{published data only\}}

NCT03075085. Developing and testing implementation strategies for evidence-based obesity prevention in childcare. clinicaltrials.gov/ct2/show/NCT03075085 (first received 9 March 2017).

NCT03279926 \{published data only\}

NCT03279926. Preschoolers Learning and Active in PlaY (PLAY). clinicaltrials.gov/ct2/show/NCT03279926 (first received 12 September 2017).

\section{NCT03590834 \{published data only\}}

NCT03590834. Míranos! program, a preschool obesity prevention RCT. ClinicalTrials.gov/show/NCT03590834 (first received 18 July 2018)

\section{NCT03695523 \{published data only\}}

NCT03695523. PLAY (PhysicaL ActivitY) policy study. clinicaltrials.gov/ct2/show/NCT03695523 (first received 4 October 2018)

\section{Yoong 2016b \{published data only\}}

Yoong SL, Grady A, Wiggers J, Flood V, Rissel C, Finch M, et al. A randomised controlled trial of an online menu planning intervention to improve childcare service adherence to dietary guidelines: a study protocol. BMJ Open 2017;7(9):e017498.

\section{Additional references}

\section{Arditi 2012}

Arditi C, Rège-Walther M, Wyatt JC, Durieux P, Burnand B. Computer-generated reminders delivered on paper to healthcare professionals; effects on professional practice and health care outcomes. Cochrane Database of Systematic Reviews 2012, Issue 12. [DOI: 10.1002/14651858.CD001175.pub3]

\section{Bilandzic 2016}

Bilandzic A, Fitzpatrick T, Rosella L, Henry D. Risk of bias in systematic reviews of non-randomized studies of adverse cardiovascular effects of thiazolidinediones and 
cyclooxygenase-2 inhibitors: application of a new Cochrane Risk of Bias tool. PLOS Medicine 2016;13(4):e1001987.

\section{Black 2013}

Black RE, Victora CG, Walker SP, Bhutta ZA, Christian P, De Onis M, et al. Maternal and Child Nutrition Study Group. Maternal and child undernutrition and overweight in low-income and middle-income countries. Lancet 2013;382(9890):427-51.

\section{Bollella 1999}

Bollella MC, Boccia LA, Nicklas TA, Lefkowitz KB, Pittman BP, Zang EA, et al. Assessing dietary intake in preschool children: the Healthy Start project - New York. Nutrition Research 1998;19(1):37-48.

\section{Brown 2019}

Brown T, Moore THM, Hooper L, Gao Y, Zayegh A, ljaz S, et al. Interventions for preventing obesity in children. Cochrane Database of Systematic Reviews 2019, Issue 7. [DOI: 10.1002/14651858.CD001871.pub4]

\section{Buller 2010}

Buller DB. Continuing issues for dissemination and implementation of cancer prevention interventions. American Journal of Preventive Medicine 2010;38(4):462-3.

\section{Cane 2012}

Cane J, O'Connor D, Michie S. Validation of the theoretical domains framework for use in behaviour change and implementation research. Implementation Science 2012;7:1-17. [DOI: 10.1186/1748-5908-7-37]

\section{Cashmore 2008}

Cashmore AW, Jones SC. Growing up active: a study into physical activity in long day care centers. Journal of Research in Childhood Education 2008;12:179-91. [DOI: 10.1080/02568540809594654]

\section{Cochrane Public Health Group 2011}

Cochrane Public Health Group. Guide for developing a Cochrane protocol. ph.cochrane.org/sites/ph.cochrane.org/files/uploads/ Guide\%20for\%20PH\%20protocol_Nov\%202011_final\%20for \%20website.pdf (accessed prior to 14 December 2019).

\section{Cohen 2015}

Cohen AN, Hamilton AB, Ritchie M, Mittman BS, Kirchner JE, Wyatt GE, et al. Improving care quality through hybrid implementation/effectiveness studies: best practices in design, methods, and measures. Implementation Science 2015;10 Suppl 1(A29):1-3. [DOI: 10.1186/1748-5908-10-S1-A29]

\section{Commonwealth of Australia}

Commonwealth of Australia. Get up and grow healthy eating and physical activity for early childhood. www.health.gov.au/ internet/main/publishing.nsf/Content/phd-gug-staffcarers (accessed prior to 14 December 2019).

\section{Copeland 2011}

Copeland KA, Kendeigh CA, Saelens BE, Kalkwarf HJ, Sherman SN. Physical activity in child-care centers: do teachers hold the key to the playground?. Health Education Research 2012;27(1):81-100.

\section{D'Agostino 1999}

D'Agostino C, D'Andrea T, Lieberman L, Sprance L, Williams CL. Healthy Start: a new comprehensive preschool health education program. Journal of Health Education 1999;30(1):9-12.

\section{Damschroder 2009}

Damschroder LJ, Aron DC, Keith RE, Kirsh SR, Alexander JA, Lowery JC. Fostering implementation of health services research findings into practice: a consolidated framework for advancing implementation science. Implementation Science 2009;4(1):50.

\section{Dobbins 2013}

Dobbins M, Husson H, DeCorby K, LaRocca RL. Schoolbased physical activity programs for promoting physical activity and fitness in children and adolescents aged 6 to 18 . Cochrane Database of Systematic Reviews 2013, Issue 2. [DOI: 10.1002/14651858.CD007651.pub2]

\section{EPOC 2015}

Effective Practice, Organisation of Care (EPOC). EPOC taxonomy. epoc.cochrane.org/epoc-taxonomy (accessed prior to 14 December 2019).

\section{Finch 2015}

Finch M, Yoong SL, Thomson RJ, Seward K, Cooney M, Jones $\mathrm{J}$, et al. A pragmatic randomised controlled trial of an implementation intervention to increase healthy eating and physical activity promoting policies and practice in centre based childcare service: study protocol. BMJ Open 2015;5:e006706.

\section{Finch 2016}

Finch M, Jones J, Yoong S, Wiggers J, Wolfenden L. Effectiveness of centre-based childcare interventions in increasing child physical activity: a systematic review and metaanalysis for policymakers and practitioners. Obesity Reviews 2016;17(5):412-28.

\section{Finucane 2011}

Finucane MM, Stevens GA, Cowan MJ, Danaei G, Lin JK, Paciorek CJ, et al. National, regional, and global trends in body-mass index since 1980: systematic analysis of health examination surveys and epidemiological studies with 960 country-years and 9.1 million participants. Lancet 2011;377:557-67.

\section{Forsetlund 2009}

Forsetlund L, Bjørndal A, Rashidian A, Jamtvedt G, O'Brien MA, Wolf FM, et al. Continuing education meetings and workshops: effects on professional practice and health care outcomes. Cochrane Database of Systematic Reviews 2009, Issue 2. [DOI: 10.1002/14651858.CD003030.pub2]

\section{Frampton 2014}

Frampton AM, Sisson SB, Horm D, Campbell JE, Lora K, Ladner JL. What's for lunch? An analysis of lunch menus in 83

Strategies to improve the implementation of healthy eating, physical activity and obesity prevention policies, practices or programmes 
urban and rural Oklahoma child-care centers providing all-day care to preschool children. Journal of the Academy of Nutrition and Dietetics 2014;114(9):1367-74.

\section{French 2012}

French SD, Green SE, O'Connor DA, McKenzie JE, Francis JJ, Michie $S$, et al. Developing theory-informed behaviour change interventions to implement evidence into practice: a systematic approach using the Theoretical Domains Framework. Implementation Science 2012;7(1):1-8. [DOI: 10.1186/1748-5908-7-38]

\section{Froehlich Chow 2011}

Froehlich Chow A, Humbert L. Physical activity and nutrition in early years care centers: barriers and facilitators. Canadian Children 2011;36(1):26-30.

\section{Gerritsen 2016}

Gerritsen S, Morton SMB, Wall CR. Physical activity and screen use policy and practices in childcare: results from a survey of early childhood education services in New Zealand. Australian and New Zealand Journal of Public Health 2016;40(4):319-25.

\section{Giguère 2012}

Giguère $A$, Légaré $F$, Grimshaw J, Turcotte $S$, Fiander $M$, Grudniewicz A, et al. Printed educational materials: effects on professional practice and healthcare outcomes. Cochrane Database of Systematic Reviews 2012, Issue 10. [DOI: 10.1002/14651858.CD004398.pub3]

\section{Glasgow 2012}

Glasgow RE, Vinson C, Chambers D, Khoury MJ, Kaplan RM, Hunter C. National Institutes of Health approaches to dissemination and implementation science; current and future directions. American Journal of Public Health 2012;107(7):1274-81.

\section{Grady 2018}

Grady A, Seward K, Finch M, Fielding A, Stacey F, Jones J, et al. Barriers and enablers to implementation of dietary guidelines in early childhood education centers in Australia: application of the Theoretical Domains Framework. Journal of Nutrition Education and Behavior 2018;50(3):229-37.e1.

\section{Gray 2009}

Gray V, Holman CDJ. Deaths and premature loss of life caused by overweight and obesity in Australia in 2011-2050: benefits from different intervention scenarios. Report for the Australian Preventive Health Taskforce. www.researchgate.net/ publication/242562415_Deaths_and_premature_loss_of_ life_caused_by_overweight_and_obesity_in_Australia_in_ 2011-_2050_Benefits_from_different_intervention_scenarios (accessed prior to 14 December 2019).

\section{Guyatt 2010}

Guyatt GH, Oxman AD, Schünemann HJ, Tugwell P, Knotterus A. GRADE guidelines: a new series of articles in the Journal of Clinical Epidemiology. Journal of Clinical Epidemiology 2011;64(4):380-2.

\section{Higgins 2011}

Higgins JPT, Green S (editors). Cochrane Handbook for Systematic Reviews of Interventions Version 5.1.0 [updated March 2011]. The Cochrane Collaboration, 2011. Available from www.handbook.cochrane.org.

\section{Higgins 2019}

Higgins JPT, Thomas J, Chandler J, Cumpston M, Li T, Page MJ, Welch VA (editors). Cochrane Handbook for Systematic Reviews of Interventions Version 6 [updated July 2019]. The Cochrane Collaboration, 2019. Available from www.training.cochrane.org/ handbook.

\section{Ivers 2012}

Ivers N, Jamtvedt G, Flottorp S, Young JM, OdgaardJensen J, French SD, et al. Audit and feedback: effects on professional practice and healthcare outcomes. Cochrane Database of Systematic Reviews 2012, Issue 6. [DOI: 10.1002/14651858.CD000259.pub3]

\section{Jaime 2009}

Jaime PC, Lock K. Do school based food and nutrition policies improve diet and reduce obesity?. Preventive Medicine 2009;48(1):45-53.

\section{Kelly 2010}

Kelly B, Hardy LL, Howlett S, King L, Farrell L, Hattersley L. Opening up Australian preschoolers' lunchboxes. Australian and New Zealand Journal of Public Health 2010;34:288-92.

\section{Lee 2018}

Lee H, Hall A, Nathan N, Reilly KL, Seward K, Williams CM, et al. Mechanisms of implementing public health interventions: a pooled causal mediation analysis of randomised trials. Implementation Science 2018;13(1):42.

\section{Mazza 2013}

Mazza D, Bairstow P, Buchan H, Paubrey Chakraborty S, Van Hecke O, Grech C, et al. Refining a taxonomy for guideline implementation: results of an exercise in abstract classification. Implementation Science 2013;8(32):1-10. [DOI: 10.1186/1748-5908-8-32]

\section{McCrabb 2019}

McCrabb S, Lane C, Hall A, Milat A, Bauman A, Sutherland R, et al. Scaling-up evidence-based obesity interventions: a systematic review assessing intervention adaptations, effectiveness and quantifying the scale-up penalty. Obesity Reviews 2019;20(7):964-82.

\section{McFadyen 2018}

McFadyen T, Chai LK, Wyse R, Kingsland M, Yoong SL, ClintonMcHarg T, et al. Strategies to improve the implementation of policies, practices or programmes in sporting organisations targeting poor diet, physical inactivity, obesity, risky alcohol use or tobacco use: a systematic review. BMJ Open 2018;8:e019151.

\section{McWilliams 2009}

McWilliams C, Ball SC, Benjamin SE, Hales D, Vaughn A, Ward DS. Best-practice guidelines for physical activity at childcare. Pediatrics 2009;124:1650-9. 


\section{Michie 2008}

Michie S, Johnston M, Francis J, Hardeman W, Eccles M. From theory to intervention: mapping theoretically derived behavioural determinants to behaviour change techniques. Applied Psychology 2008;57(4):660-80.

\section{O'Brien 2007}

O'Brien MA, Rogers S, Jamtvedt G, Oxman AD, OdgaardJensen J, Kristoffersen DT, et al. Educational outreach visits: effects on professional practice and health care outcomes. Cochrane Database of Systematic Reviews 2007, Issue 4. [DOI: 10.1002/14651858.CD000409.pub2]

\section{Powell 2015}

Powell BJ, Waltz TJ, Chinman MJ, Damschroder LJ, Smith JL, Matthieu MM, et al. A refined compilation of implementation strategies: results from the Expert Recommendations for Implementing Change (ERIC) project. Implementation Science 2015;10(1):21.

\section{Proctor 2011}

Proctor E, Silmere H, Raghavan R, Hovmand P, Aarons G, Bunger A. Outcomes for implementation research: conceptual distinctions, measurement challenges, and research agenda. Administration and Policy in Mental Health 2011;38(2):65-76.

\section{Rabin 2008}

Rabin BA, Brownson RC, Haire-Joshu D, Kreuter MW, Weaver NL. A glossary for dissemination and implementation research in health. Journal of Public Health Management Practice 2008;14(2):117-23.

\section{Rabin 2010}

Rabin BA, Galsgow RE, Kerner JF, Klump MP, Brownson RC. Dissemination and implementation research on communitybased cancer prevention. American Journal of Preventive Medicine 2010;38(4):443-56.

\section{Rosemburg 2003}

Rosemburg F. Multilateral organizations and early child care and education policies for developing countries. Gender and Society 2003;17(2):250-66. [DOI: 10.1177/0891243202250831]

\section{Schillinger 2010}

Schillinger D. An introduction to effectiveness, dissemination and implementation research. In: Fleisher P, Goldstein E editor(s). UCSF Clinical and Translational Science Institute (CTSI) Resource Manuals and Guides to Community-Engaged Research. San Francisco: Clinical Translational Science Institute Community Engagement Program, University of California, 2010.

\section{Scott 2011}

Scott A, Sivey P, Ait Ouakrim D, Willenberg L, Naccarella L, Furler J, et al. The effect of financial incentives on the quality of health care provided by primary care physicians. Cochrane Database of Systematic Reviews 2011, Issue 9. [DOI: 10.1002/14651858.CD008451.pub2]

\section{Sisson 2012}

Sisson S, Campbell JE, May KB, Brittain DR, Monrie LA, Guss SH, et al. Assessment of food, nutrition, and physical activity practices in Oklahoma child-care centers. Journal of the Academy of Nutrition and Dietetics 2012;112:1230-40.

\section{Sisson 2016}

Sisson SB, Krampe M, Anundson K, Castle S. Obesity prevention and obesogenic behavior interventions in child care: a systematic review. Preventive Medicine 2016;87:57-69.

\section{Spark 1998}

Spark A, Pfau J, Nicklas TA, Williams CL. Reducing fat in preschool meals: description of the foodservice intervention component of Healthy Start. Journal of Nutrition Education 1998;30(3):170-7.

\section{Squires 2014}

Squires JE, Sullivan K, Eccles MP, Worswick J, Grimshaw JM. Are multifaceted interventions more effective than single-component interventions in changing health-care professionals' behaviours? An overview of systematic reviews. Implementation Science 2014;9(1):1.

\section{Stacey 2017}

Stacey F, Finch M, Wolfenden L, Grady A, Jessop K, Wedesweiler T, et al. Evidence of the potential effectiveness of centre-based childcare policies and practices on child diet and physical activity: consolidating evidence from systematic reviews of intervention trials and observational studies. Current Nutrition Reports 2017;6(3):228-46.

\section{Swinburn 2011}

Swinburn BA, Sacks G, Hall KD, McPherson K, Finegood DT, Moodie ML, et al. The global obesity pandemic: shaped by global drivers and local environments. Lancet 2011;378(9793):804-14.

\section{Tremblay 2012}

Tremblay L, Boudreau-Lariviere C, Cimon-Lambert K. Promoting physical activity in pre-schoolers: a review of the guidelines, barriers, and facilitators for implementation of policies and practices. Canadian Psychology 2012;53(4):280-90.

\section{Virgara 2019}

Virgara R, Maher C, Lewis LK, Phillips A, Wolfenden L, Okely A, et al. Interventions in outside-school hours childcare settings for promoting physical activity amongst schoolchildren aged 4 to 12 years. Cochrane Database of Systematic Reviews 2019, Issue 7. [DOI: 10.1002/14651858.CD013380]

\section{Wang 2011}

Wang YC, McPherson K, Marsh T, Gortmaker SL, Brown M. Health and economic burden of the projected obesity trends in the USA and the UK. Lancet 2011;378:815-25.

\section{Waters 2011}

Waters E, De Silva-Sanigorski A, Burford BJ, Brown T, Campbell KJ, Gao Y, et al. Interventions for preventing obesity in children. Cochrane Database of Systematic Reviews 2011, Issue 12. [DOI: 10.1002/14651858.CD001871.pub3] 


\section{Williams 1998}

Williams CL, Squillace MM, Bollela MC, Brotanek J, Campanaro L, D'Agostino C, et al. Healthy Start: a comprehensive health education program for preschool children. Preventive Medicine 1998;27:216-23.

\section{Williams 2004}

Williams CL, Strobino BA, Bollela M, Brotanek J. Cardiovascular risk reduction in preschool children: the "Healthy Start" project. Journal of the American College of Nutrition 2004;23(2):117-23. [DOI: 10.1080/07315724.2004.10719351]

\section{Wilson 2017}

Wilson PM, Sales A, Wensing M, Aarons GA, Flottorp S, Glidewell $\mathrm{L}$, et al. Enhancing the reporting of implementation research. Implementation Science 2017;12(1):13.

\section{Wolfenden 2015a}

Wolfenden L, FInch M, Nathan N, Weaver N, Wiggers J, Yoong SL, et al. Factors associated with early childhood education and care service implementation of healthy eating and physical activity policies and practices in Australia: a cross-sectional study. Translational Behavioural Medicine 2015;5(3):327-34.

\section{Wolfenden 2016}

Wolfenden L, Milat AJ, Lecathelinais C, Skelton E, ClintonMcHarg T, Williams C, et al. A bibliographic review of public health dissemination and implementation research output and citation rates. Preventive Medicine Reports 2016;4:441-3.

\section{Wolfenden 2016a}

Wolfenden L, Milat AJ, Lecathelinais C, Sanson-Fisher RW, Carey ML, Bryant $\mathrm{J}$, et al. What is generated and what is used: a description of public health research output and citation. European Journal of Public Health 2016;26(3):523-5.

\section{Wolfenden 2016b}

Wolfenden L, Finch M, Wyse R, Clinton-McHarg T, Yoong SL. Time to focus on implementation: the need to re-orient research on physical activity in childcare services. Australian and New Zealand Journal of Public Health 2016;40(3):209-10.

\section{Wolfenden 2016c}

Wolfenden L, Milat AJ, Lecathelinais C, Skelton E, Clinton$\mathrm{McHarg} \mathrm{T}$, Williams C, et al. A bibliographic review of public health dissemination and implementation research output and citation rates. Preventive Medicine Reports 2016;4:441-3.

\section{Wolfenden 2017}

Wolfenden L, Nathan NK, Sutherland R, Yoong SL, Hodder RK, Wyse RJ, et al. Strategies for enhancing the implementation of school-based policies or practices targeting risk factors for chronic disease. Cochrane Database of Systematic Reviews 2017, Issue 11. [DOI: 10.1002/14651858.CD011677.pub2]

\section{Wolfenden 2018}

Wolfenden L, Goldman S, Stacey FG, Grady A, Kingsland M, Williams CM, et al. Strategies to improve the implementation of workplace-based policies or practices targeting tobacco, alcohol, diet, physical activity and obesity. Cochrane Database of Systematic Reviews 2018, Issue 11. [DOI: 10.1002/14651858.CD012439.pub2]

\section{Wolfenden 2019}

Wolfenden L, Reilly K, Kingsland M, Grady A, Williams CM, Nathan N, et al. Identifying opportunities to develop the science of implementation for community-based non-communicable disease prevention: a review of implementation trials. Preventive Medicine 2019;118:279-85.

\section{World Health Organization 2012}

World Health Organization. Population based approaches to childhood obesity prevention. www.who.int/ dietphysicalactivity/childhood/WHO_new_childhoodobesity_ PREVENTION_27nov_HR_PRINT_OK.pdf (accessed prior to 15 December 2019).

\section{World Health Organization 2018}

World Health Organization. Obesity and overweight 2018. www.who.int/news-room/fact-sheets/detail/obesity-andoverweight (accessed prior to 14 December 2019).

\section{Yoong 2014}

Yoong SL, Skelton E, Jones J, Wolfenden L. Do childcare services provide foods in line with the 2013 Australian Dietary guidelines? A cross-sectional study. Australian and New Zealand Journal of Public Health 2014;38(6):595-6.

\section{Yoong 2014b}

Yoong SL, Wolfenden L, Clinton-McHarg T, Waters E, Pettman TL, Steele $\mathrm{E}$, et al. Exploring the impact of pragmatic and explanatory study designs on outcomes of systematic reviews of public health interventions: a case study. Journal of Public Health 2014;36(1):170-6.

\section{Yoong 2015}

Yoong SL, Clinton-Mcharg T, Wolfenden L. Systematic reviews examining implementation of research into practice and impact on population health are needed. Journal of Clinical Epidemiology 2015;68(7):788-91.

\section{Zoritch 2000}

Zoritch B, Roberts I, Oakley A. Day care for pre-school children. Cochrane Database of Systematic Reviews 2000, Issue 3. [DOI: 10.1002/14651858.CD000564]

\section{References to other published versions of this review Wolfenden 2015b}

Wolfenden L, Jones J, Finch M, Wyse RJ, Yoong SL, Steele EJ, et al. Strategies to improve the implementation of healthy eating, physical activity and obesity prevention policies, practices or programmes within childcare services. Cochrane Database of Systematic Reviews 2015, Issue 7. [DOI: 10.1002/14651858.CD011779]

* Indicates the major publication for the study 


\title{
CHARACTERISTICS OF STUDIES
}

Characteristics of included studies [ordered by study ID]

Alkon 2014

Study design: Cluster-RCT
Intervention duration: 7 months
Length of follow-up from baseline: 7 months
Differences in baseline characteristics: reported
Unit of allocation: childcare service
Unit of analysis: childcare service (child behaviour and weight status were assessed at the level of the
individual)

Participants

\author{
Service type: childcare centres \\ Region: California, Connecticut and North Carolina, USA \\ Demographic/socioeconomic characteristics: children between the ages of 3 and 5 years of age from \\ racial/ethnically diverse backgrounds and primarily of low-income families \\ Inclusion/exclusion criteria: inclusion criteria: English-speaking service manager, on-site kitchen, \\ racial/ethnic diversity among the children, participation by at least $60 \%$ of families, and a population of \\ children in care primarily comprised of low-income children between the ages of 3 and 5 years of age \\ Number of services randomised: 18 (9 intervention, 9 control) \\ Numbers by trial group:
}

$\mathrm{n}$ (controls baseline) $=9$

$\mathrm{n}$ (controls follow-up) $=9$ ( 2 small services under same ownership analysed as 1 service)

$\mathrm{n}$ (interventions baseline) $=9$

$\mathrm{n}$ (interventions follow-up) $=9$

\section{Recruitment:}

Service: 42 childcare services were recruited, of which 24 services did not meet the inclusion criteria. Childcare health consultants from California and North Carolina recruited the convenience sample of services for their respective states while Connecticut services were recruited by the Connecticut principal investigator.

\section{Child:}

Physical activity: 8 children at each service, randomly selected by a statistician BMI: the research assistants selected children at the pre-intervention period for height and weight measurements from service-specific randomly ordered lists of enrolled children. Those with pre-intervention measurements (268) were prioritised for measurement post-intervention (336); 209 children had useable data at both time points.

Recruitment rate: $43 \%$

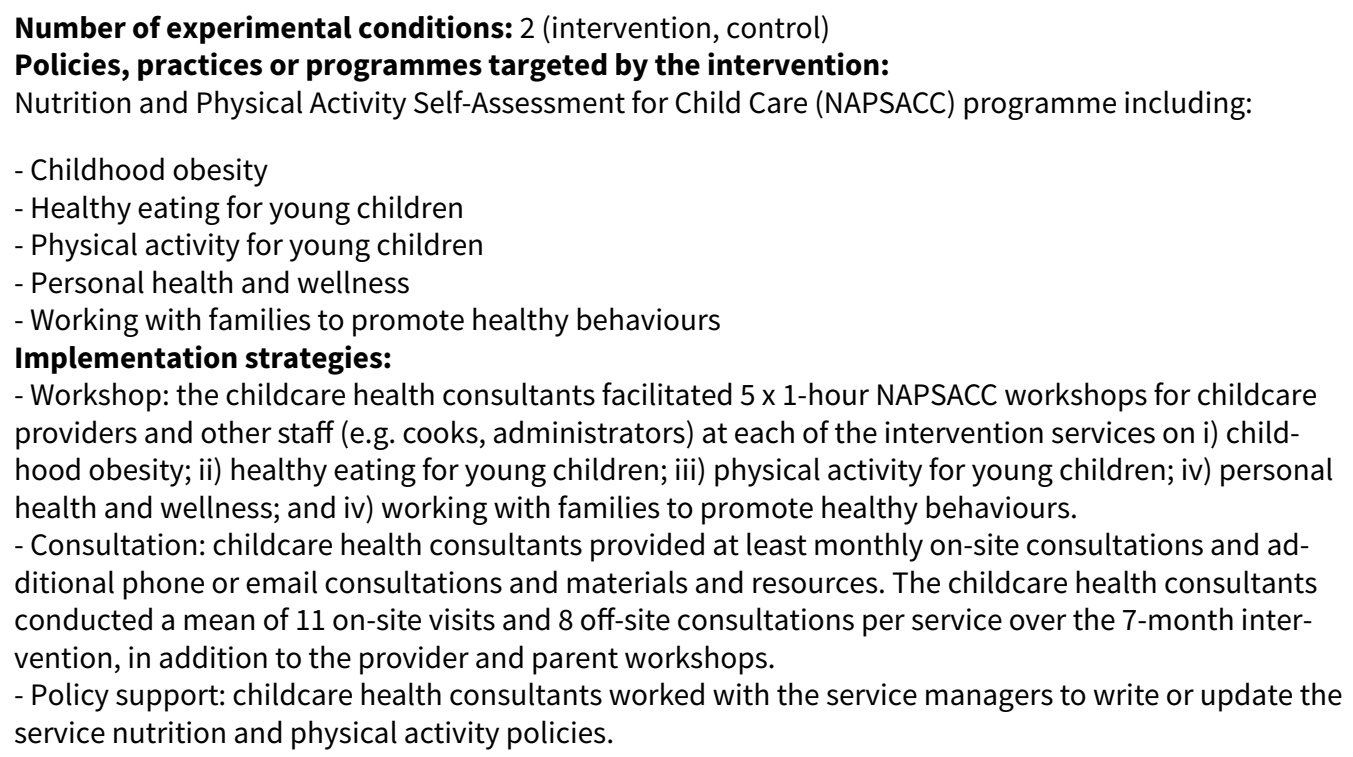


Alkon 2014 (Continued)

- Parent workshop: 7 of the intervention services also received the parent workshop "Raising Healthy Kids".

Who delivered the intervention: previously trained nurse childcare health consultants

Theoretical underpinning: not reported

Description of control: delayed NAPSACC intervention in year 2 of the study

Outcomes

Outcome relating to the implementation of childcare service policies, practices or programmes: Service nutrition and physical activity policies:

Data collection method: Californian Childcare Health Programme Health and Safety Checklist $(\mathrm{CH}-$

PHSPC) completed by blinded research assistants and used to determine if the service's written policies adhered to national guidelines

Validity of measures used: unclear - this policy measurement technique was used in another study and was shown to be a valid measure of the effect of childcare health consultant interventions on childcare service environments.

Provider nutrition and physical activity practices:

Data collection method: modified version of the Environment and Policy Assessment and Observation (EPAO) was completed by a research assistant. Mean scores for the nutrition and physical activity scales were calculated for each service then aggregated by intervention and control services.

Validity of measures used: although these items were modified from a reliable instrument, they were not previously validated in the format included in this study.

Outcome relating to cost: not applicable

Outcome relating to adverse consequences: not applicable

Outcome relating to child diet, physical activity or weight status:

Child physical activity:

Data collection method: the Observation System for Recording Activity in Preschools (OSRAP) - data collection was completed by a trained research assistant. Children were observed in 15-second intervals for a total of 12 to 16 minutes per child; the observations were conducted over an 8-hour day. Data were aggregated as the mean percentage of physical activity intensity ( $1=$ stationary to $5=$ fast). Validity of measures used: the OSRAP has been validated and has been compared favourably with accelerometer data.

\section{Child weight status:}

Data collection method: BMI z-score - the research assistants used a portable foldable stadiometer to measure height and a digital scale to measure weight. Pre/post BMI z-score and \% underweight, healthy weight, overweight and obese children

Validity of measures used: unclear - appears to be an objective measure

Outcome relating to implementation strategy acceptability, adoption, penetration, sustainability and appropriateness:

\section{Penetration:}

Data collection method: intervention receipt

Validity of measures used: not reported vices, Health Resources and Services Administration, Maternal and Child Health Research Program.

\section{Risk of bias}

Bias Authors' judgement Support for judgement

Random sequence genera- Unclear risk tion (selection bias)

\begin{abstract}
Authors indicated that the services were randomly assigned to treatment groups, but the sequence generation procedure was not described.

One control group service that was not able to adequately complete baseline data collection was replaced by a matched service (unclear if this was randomly chosen).
\end{abstract}


Alkon 2014 (Continued)

Allocation concealment Unclear risk Method of concealment not described
(selection bias)

\begin{tabular}{|c|c|c|}
\hline $\begin{array}{l}\text { Blinding of participants } \\
\text { and personnel (perfor- } \\
\text { mance bias) }\end{array}$ & High risk & $\begin{array}{l}\text { We assumed that due to the nature of the intervention, childcare service staff } \\
\text { and study personnel delivering the intervention were not blind to the study al- } \\
\text { location and therefore there was a potential high risk of performance bias. }\end{array}$ \\
\hline
\end{tabular}

\begin{tabular}{lll}
\hline $\begin{array}{l}\text { Blinding of outcome as- } \\
\text { sessment (detection bias) } \\
\text { All outcomes }\end{array}$ & Low risk & $\begin{array}{l}\text { Outcome assessment was undertaken by blinded research personnel and } \\
\text { therefore the risk of detection bias was considered to be low. }\end{array}$ \\
\hline $\begin{array}{l}\text { Incomplete outcome data } \\
\begin{array}{l}\text { (attrition bias) } \\
\text { All outcomes }\end{array}\end{array}$ & Low risk & $\begin{array}{l}\text { Complete data collected for all services (8 control and } 9 \text { intervention), with no } \\
\text { services excluded from the analysis - therefore risk of attrition bias was consid- } \\
\text { ered to be low. }\end{array}$ \\
\hline
\end{tabular}

\begin{tabular}{|c|c|c|}
\hline $\begin{array}{l}\text { Selective reporting (re- } \\
\text { porting bias) }\end{array}$ & Unclear risk & $\begin{array}{l}\text { No prospective trial protocol or trial registration so it was unclear whether } \\
\text { there was selective outcome reporting. }\end{array}$ \\
\hline
\end{tabular}

\begin{tabular}{lll}
\hline Recruitment to cluster & Low risk & $\begin{array}{l}\text { Selection of participants from each service for measurement of child diet, } \\
\text { physical activity and BMI outcomes was random, so risk of bias through selec- } \\
\text { tion to cluster was considered to be low. }\end{array}$ \\
\hline Baseline imbalance & Unclear risk & $\begin{array}{l}\text { There was baseline imbalance in parent and childcare provider characteristics } \\
\text { but they adjusted for some of these in the analysis. }\end{array}$ \\
\hline Loss of clusters & Unclear risk & $\begin{array}{l}\text { In the control group, the investigators replaced 1 cluster with a matched clus- } \\
\text { ter and then merged 2 clusters (services that came under same management) } \\
\text { for analysis. }\end{array}$ \\
\hline $\begin{array}{l}\text { Incorrect analysis } \\
\begin{array}{l}\text { Compatibility with individ- } \\
\text { ually randomised RCTs }\end{array}\end{array}$ Unclear risk & $\begin{array}{l}\text { Hierarchical linear models conducted to assess child-level BMI z-score out- } \\
\text { comes (accounting for clustering within the service) }\end{array}$ \\
\hline
\end{tabular}

\section{Bell 2014}

$\begin{array}{ll}\text { Methods } & \text { Study design: nonrandomised trial } \\ \text { Intervention duration: average of } 22 \text { months between initiation of intervention and collection of fol- } \\ \text { low-up data } \\ \text { Length of follow-up from baseline: average } 22 \text { months (between initiation of intervention and collec- } \\ \text { tion of follow-up data) } \\ \text { Differences in baseline characteristics: reported } \\ \text { Unit of allocation: childcare service } \\ \text { Unit of analysis: childcare service }\end{array}$

Service type: preschools and long daycare services
Region: Intervention: Hunter New England region, New South Wales, Australia; Control: New South
Wales, Australia
Demographic/socioeconomic characteristics: Intervention: the Hunter New England region - a geo-
graphically large area $\left(130,000 \mathrm{~km}^{2}\right)$ with a demographically diverse population including metropoli-
tan urban and suburban areas, regional services, and rural and isolated remote communities. The re-
gion included pockets of wealth and poverty, and an overall socioeconomic status lower than the New
South Wales state average. Control: not reported

Strategies to improve the implementation of healthy eating, physical activity and obesity prevention policies, practices or programmes 
Inclusion/exclusion criteria: all services located within the intervention region were invited to participate. Services were excluded that catered for children with special needs such as intellectual or physical disabilities.

Number of services randomised: 583 (287 intervention, 296 control) Numbers by trial group:

$\mathrm{n}$ (control baseline $)=251$

$\mathrm{n}$ (control follow-up) $=191$

$\mathrm{n}$ (intervention baseline) $=261$

$\mathrm{n}$ (intervention follow-up) $=240$

Recruitment: Intervention: all services $(n=287)$ located within the intervention region were invited to participate. Control: a simple random sample of eligible centre-based childcare services in all other regions of the state of New South Wales were invited to participate in the study as the comparison group $(n=296)$

Recruitment rate: Intervention: 91\%; Control: $85 \%$

Interventions

Number of experimental conditions: 2 (intervention, control)

Policies, practices or programmes targeted by the intervention:

Healthy eating policies and practices of childcare services including:

- Staff training in nutrition

- Policy guiding the content of food and drinks provided to children by the service

- Policy guiding the content of food and drinks packed for children by parents

- Provision of non-sweetened drinks (milk and water) only to children during care

- Parent participation in nutrition policy or programmes

- Provision of foods to children consistent with dietary guidelines (for services that provide meals to children) and accreditation requirements

Implementation strategies:

- Identifying leaders and obtaining their support and endorsement of the programme and targeted policy and practices

- Provision of professional development for staff ( 2 × 6-hour workshops)

- Small incentives

- Resource provision

- Performance monitoring and feedback

- Follow-up support (20-minute phone call once, 5 newsletters)

Who delivered the intervention: Health service staff who worked with regional representatives of the Department of Community Services and childcare service staff to implement the intervention strategies

Theoretical underpinning: The intervention was based on practice change and capacity-building theoretical frameworks.

Description of control: From July 2008 onwards, preschool services in New South Wales were able to access implementation support via a government-supported programme that aimed to promote physical activity and healthy eating for children.

Outcomes

Outcome relating to the implementation of childcare service policies, practices or programmes: Service healthy eating policies and practices:

- Staff with nutrition training

- Services with a policy guiding the content of food and drinks provided to children by the service

- Services with a policy guiding the content of food and drinks packed for children by parents

- Services providing only water or plain milk to children

- Parent participation in nutrition policy or programmes

Data collection method: computer-assisted telephone interview with service managers

Validity of measures used: not reported

\section{Nutritional quality of lunch menus:}

- Number of times processed foods high in fat, salt and/or sugar were listed on the menu each day

- Number of times sweetened drinks were listed on the menu each day

- Number of times water was listed on the menu each day

- Number of 'child size' servings of fruit listed on the menu each day

- Number of 'child size' servings of vegetables listed on the menu each day

Classification into the following categories:

- No high-fat, salt and/or sugar processed food menu items

- No sweetened drink menu items

Strategies to improve the implementation of healthy eating, physical activity and obesity prevention policies, practices or programmes 
Bell 2014 (Continued)

\author{
- Water with every eating occasion \\ - 1 child-size serving of fruit listed on the menu each day \\ - The number of child-size servings of vegetables listed on the menu each day \\ Data collection method: All services were invited to submit a copy of their current 2-week menu. \\ Validity of measures used: not reported \\ Outcome relating to cost: not applicable \\ Outcome relating to adverse consequences: not applicable \\ Outcome relating to child diet, physical activity or weight status: not applicable
}

Outcome relating to implementation strategy acceptability, adoption, penetration, sustainability and appropriateness:

not applicable

\begin{tabular}{ll}
\hline Notes & - \\
\hline
\end{tabular}

\title{
Risk of bias
}

Bias Authors' judgement Support for judgement

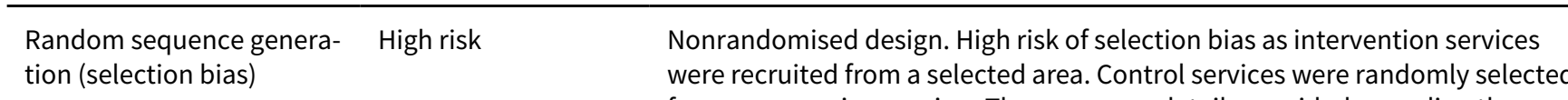
from a comparison region. There were no details provided regarding the sequence generation procedure used to randomise control services for selection.

\begin{tabular}{|c|c|c|}
\hline $\begin{array}{l}\text { Allocation concealment } \\
\text { (selection bias) }\end{array}$ & High risk & $\begin{array}{l}\text { Nonrandomised experimental design. Intervention services were recruited } \\
\text { from a selected area, therefore high risk of bias as no concealment of alloca- } \\
\text { tion. }\end{array}$ \\
\hline
\end{tabular}

\begin{tabular}{|c|c|c|}
\hline $\begin{array}{l}\text { Blinding of participants } \\
\text { and personnel (perfor- } \\
\text { mance bias) }\end{array}$ & High risk & $\begin{array}{l}\text { We assumed that, due to the nature of the intervention, childcare service staff } \\
\text { and study personnel delivering the intervention were not blind to the study al- } \\
\text { location and therefore there was a potential high risk of performance bias. }\end{array}$ \\
\hline
\end{tabular}

All outcomes

\begin{tabular}{lll}
\hline $\begin{array}{l}\text { Blinding of outcome as- } \\
\text { sessment (detection bias) }\end{array}$ & High risk & $\begin{array}{l}\text { Self-reported policies and practices. There was no blinding of research person- } \\
\text { nel or } \\
\text { All outcomes }\end{array}$ \\
& $\begin{array}{l}\text { participants (service managers) and due to the self-report of this outcome, } \\
\text { risk of bias was considered to be high. }\end{array}$
\end{tabular}

Incomplete outcome data High risk (attrition bias)

All outcomes

There was a large difference in the proportion of services followed up amongst intervention and control groups and the proportion that provided a menu for assessment:

Intervention group: $91 \%$ of services surveyed at baseline were followed up and $61 \%$ provided a menu.

Control group: $76 \%$ of services from the control area (NSW) were followed up and $49 \%$ provided a menu.

Due to the magnitude of difference in the proportions of participants followed up between groups, the risk of bias was assessed as high.

\begin{tabular}{lll}
\hline $\begin{array}{l}\text { Selective reporting (re- } \\
\text { porting bias) }\end{array}$ & Unclear risk & $\begin{array}{l}\text { No prospective trial protocol or trial registration so it was unclear whether } \\
\text { there was selective outcome reporting. }\end{array}$ \\
\hline Potential confounding & Unclear risk & $\begin{array}{l}\text { Authors stated that "Characteristics of services were not adjusted for in the lo- } \\
\text { gistic regression model as we were looking at change within services and the } \\
\text { baseline score of the services effectively controlled for potential differences in } \\
\text { baseline characteristics between the two regions." It is unknown whether this } \\
\text { was adequate to reduce bias due to known confounders. }\end{array}$
\end{tabular}

Strategies to improve the implementation of healthy eating, physical activity and obesity prevention policies, practices or programmes 
Bell 2014 (Continued) $\begin{array}{ll}\text { Other bias } & \text { Unclear risk } \\ & \text { This research was funded by NSW Ministry of Health. The Ministry of Health } \\ \text { had no role in the design, analysis or writing of this article. }\end{array}$

Benjamin 2007

Study design: Cluster-RCT
Intervention duration: 6 months
Length of follow-up from baseline: approximately 10 months (assessments occurred 4 months after
the 6-month intervention)
Differences in baseline characteristics: not reported
Unit of allocation: county
Unit of analysis: childcare service

Participants Service type: childcare centres

Region: North Carolina, USA

Demographic/socioeconomic characteristics: not reported

Inclusion/exclusion criteria: inclusion criteria: size of the childcare service (between 20 and 150 children); participation in the Child and Adult Care Food Program; rating of 3, 4 or 5 stars on the NC 1-5 Star Rating System for quality childcare. Exclusion criteria: open case of child abuse or neglect; service provided services to a special population of children only; Head Start service; classified as a family childcare home

Number of services randomised: 19 (15 intervention, 4 control)

Numbers by trial group:

$\mathrm{n}$ (control baseline) $=4$

$\mathrm{n}$ (control follow-up) $=4$

$\mathrm{n}$ (intervention baseline) $=15$ ( 2 intervention services withdrew because their manager had left their position)

$\mathrm{n}$ (intervention follow-up) $=13$

Recruitment: convenience sampling - the North Carolina childcare regulatory agency provided a list of eligible childcare services for each intervention and comparison county. 2 services were selected per county, except for 1 large county where 5 services participated.

Recruitment rate: not reported

Interventions

\section{Number of experimental conditions: 2 (intervention, control) Policies, practices or programmes targeted by the intervention:}

The programme focused on 15 nutrition and physical activity areas. Nutrition areas of focus included: fruits and vegetables; fried food and high-fat meats; beverages; menus and variety; meals and snacks; food items outside of regular meals and snacks; supporting healthful eating; nutrition education for children, parents and staff; and nutrition policy. Key physical activity areas of focus included: active play and inactive time; TV use and TV viewing; play environment; supporting physical activity; physical activity education for children, parents and staff; and physical activity policy.

\section{Implementation strategies:}

- Self-assessment: childcare service managers, with assistance from key service staff, completed the self-assessment instrument to identify current service nutrition and physical activity policies and practices.

- Action plan: NAPSACC-trained childcare health consultants worked with the services to develop an action plan to improve at least 3 areas identified from the self-assessment instrument. Childcare service managers were asked to select their priority areas for improvement in order to facilitate the most fitting and lasting environmental changes at the service.

- Workshops: the trained childcare health consultants delivered $3 \times 30$-minute workshops on being overweight, healthful eating and physical activity.

- Provision of technical assistance: ongoing technical assistance (visits and calls) were provided by the childcare health consultants to service managers to support policy and practice changes.

Who delivered the intervention: NAPSACC-trained childcare health consultants

Theoretical underpinning: NAPSACC is a theory-based programme that employs components of social cognitive theory against a backdrop of the socioecological framework. Social cognitive theory identifies several factors that influence behaviour change, including expectancies, observational learning,

Strategies to improve the implementation of healthy eating, physical activity and obesity prevention policies, practices or programmes 
Benjamin 2007 (Continued)

self-efficacy, behavioural capability, reinforcement and reciprocal determinism, which were all principles used to guide the NAPSACC intervention.

Description of control: the comparison services did not receive any training or technical assistance from a childcare health consultant but completed only the pre- and post-self-assessment instrument.

Outcomes

Outcome relating to the implementation of childcare service policies, practices or programmes: Total nutrition and physical activity score assessed using the self-assessment instrument, which included 29 nutrition and 15 physical activity questions with either a demonstrated or a perceived relationship to childhood overweight. Each question had 3 response categories, assigned 1,2 or 3 points ( 1 = minimum standard, 2 = good, 3 = best practice).

Data collection method: self-assessment instrument

Validity of measures used: not established at time of study - additional work tests the reliability and validity of the NAPSACC self-assessment instrument in a sample of childcare services.

Outcome relating to cost: not applicable

Outcome relating to adverse consequences: not applicable

Outcome relating to child diet, physical activity or weight status: not applicable

Outcome relating to implementation strategy acceptability, adoption, penetration, sustainability and appropriateness:

\section{Acceptability}

Data collection method: self-report with childcare service directors collected via focus groups, questionnaires and telephone interviews

Validity of measures used: not reported

Notes Given the small sample size $(n=4)$ in the comparison group, no between-group comparisons were made.

This project was funded by the Division of Public Health, North Carolina Department of Health and Human Services.

\section{Risk of bias}

\begin{tabular}{|c|c|c|}
\hline Bias & Authors' judgement & Support for judgement \\
\hline $\begin{array}{l}\text { Random sequence genera- } \\
\text { tion (selection bias) }\end{array}$ & Unclear risk & $\begin{array}{l}\text { Counties were matched and randomly allocated to control or intervention } \\
\text { groups. The sequence generation procedure was not described. }\end{array}$ \\
\hline $\begin{array}{l}\text { Allocation concealment } \\
\text { (selection bias) }\end{array}$ & Unclear risk & Unclear as to whether concealment of allocation occurred \\
\hline $\begin{array}{l}\text { Blinding of participants } \\
\text { and personnel (perfor- } \\
\text { mance bias) } \\
\text { All outcomes }\end{array}$ & High risk & $\begin{array}{l}\text { We assumed that due to the nature of the intervention, childcare service staff } \\
\text { and study personnel delivering the intervention were not blind to the study al- } \\
\text { location and therefore there was a potential high risk of performance bias. }\end{array}$ \\
\hline $\begin{array}{l}\text { Blinding of outcome as- } \\
\text { sessment (detection bias) } \\
\text { All outcomes }\end{array}$ & High risk & $\begin{array}{l}\text { Self-assessment conducted by childcare service staff for nutrition and physical } \\
\text { activity } \\
\text { policies and practices } \\
\text { No blinding of research personnel or participants (service managers) and due } \\
\text { to the self-report of this outcome, the risk of bias was considered high. }\end{array}$ \\
\hline $\begin{array}{l}\text { Incomplete outcome data } \\
\text { (attrition bias) } \\
\text { All outcomes }\end{array}$ & Unclear risk & $\begin{array}{l}17 \text { of the } 19 \text { intervention group services had full data available and } 4 \text { of } 4 \text { con- } \\
\text { trol services. No information was provided on the characteristics of the ser- } \\
\text { vices that dropped out, nor sensitivity analysis undertaken to test assump- } \\
\text { tions regarding missing data. }\end{array}$ \\
\hline
\end{tabular}


Benjamin 2007 (Continued)

Selective reporting (re- Unclear risk No prospective trial protocol or trial registration so it was unclear whether porting bias) there was selective outcome reporting.

\begin{tabular}{ll}
\hline Recruitment to cluster $\quad$ Unclear risk & $\begin{array}{l}\text { All services within the county invited to participate and chosen to participate } \\
\text { on first-come basis }-2 \text { per county, but } 1 \text { county was given permission to have } 5 \\
\text { services participate. }\end{array}$
\end{tabular}

Baseline imbalance Unclear risk A convenience sample of 6 intervention and 2 comparison counties, matched on urban/rural status randomly allocated to intervention or comparison group. Unclear if baseline characteristic imbalances were present as this was not reported. Outcome measures at baseline were similar.

\begin{tabular}{lll}
\hline Loss of clusters & Unclear risk & Unclear whether the 2 lost services were from the same county \\
\hline Incorrect analysis & High risk & No statistical analysis completed due to small sample size \\
\hline $\begin{array}{l}\text { Compatibility with individ- } \\
\text { ually randomised RCTs }\end{array}$ & Unclear risk & Unable to determine if a herd effect existed \\
\hline
\end{tabular}

Esquivel 2016

Methods Study design: Intervention trial within a larger RCT

Intervention duration: 7 months

Length of follow-up from baseline: 1 year

Differences in baseline characteristics: reported

Unit of allocation: childcare service

Unit of analysis: childcare service

\title{
Participants
}

\author{
Service type: centre \\ Region: 2 communities on O'ahu, Hawaii
}

Demographic/socioeconomic characteristics: Head Start (HS) is a federally funded preschool programme serving low-income children aged 3-5 years within remote underserved minority populations in the Pacific region.

\author{
Inclusion/exclusion criteria: Not described \\ Number of services randomised: 23 centres \\ Numbers by trial group: \\ $\mathrm{n}$ (controls baseline) $=12$ \\ $\mathrm{n}$ (controls follow-up) $=11$ \\ $\mathrm{n}$ (interventions baseline $)=12$ \\ $\mathrm{n}$ (interventions follow-up) $=11$
}

\section{Recruitment:}

Service: This research was embedded within the randomised community trial, the Children's Healthy Living Program for Remote Underserved Minority Populations in the Pacific Region. Total of $23 \mathrm{HS}$ classrooms from 18 HS joined the study. 
Child: Child sample included 349 children from the 23 classes from 18 centres $(n=173$ intervention, $n=$ 176 delayed intervention).

Recruitment rate: not reported

Interventions

Number of experimental conditions: 2 (intervention, waiting-list control)

Policies, practices or programmes targeted by the intervention:

- Nutrition and physical activity environment

- Meal service style and types of foods and beverages served teachers in implementing wellness policies to promote nutrition and PA in their classrooms

- To affect multiple contributing factors to the availability of foods high in sugar and fat, classroom activities and practices, and social norms.

\section{Implementation strategies:}

Educational materials: Classroom resources from the Healthy Habits for Life curriculum

Educational meetings: Training and technical assistance

Other: Monthly employee wellness activities that reinforced their role as models for healthy eating and $\mathrm{PA}$ in the classroom

Who delivered the intervention: Staff members for policy component, but unclear for staff health component

Theoretical underpinning: Social ecological model

Description of control: Waiting-list control (delayed intervention)

Data collection method: Environment and Policy Assessment and Observations (EPAO) of the classroom environment

Validity of measures used: EPAO is a validated tool.

Outcome relating to cost: not applicable

Outcome relating to adverse consequences: not applicable

Outcome relating to child diet, physical activity or weight status:

\section{Child BMI:}

Data collection method: Child height was measured by a stadiometer to the nearest $0.1 \mathrm{~cm}$. Child weight was measured using a portable scales to the nearest $0.1 \mathrm{~kg}$. BMI was calculated using the measured mean height and weight.

Validity of measures used: Child BMI variables were calculated based on 2000 CDC Growth Charts, BMI for Age and Sex. zBMI and change in zBMI over the programme year were calculated to measure change in BMI status, adjusting for age and sex.

\section{Child dietary intake:}

Data collection method: Dietary intake of children was assessed by observed plate waste, as recommended by the IOM's plan for measuring obesity prevention efforts.

Validity of measures used: not reported 
Esquivel 2016 (Continued)

Outcome relating to implementation strategy acceptability, adoption, penetration, sustainability and appropriateness: not applicable

Notes

This project was supported by the Agriculture and Food Research Initiative, Grant No.

2011-68001-30335 from the USA Department of Agriculture, National institute of Food and Agricultural

Science Enhancement Coordinated Agricultural Program.

\section{Risk of bias}

\begin{tabular}{lll}
\hline Bias & Authors' judgement & Support for judgement \\
\hline $\begin{array}{l}\text { Random sequence genera- } \\
\text { tion (selection bias) }\end{array}$ & Unclear risk & The random sequence generation procedure was not described. \\
\hline $\begin{array}{l}\text { Allocation concealment } \\
\text { (selection bias) }\end{array}$ & Unclear risk & $\begin{array}{l}\text { There was no information provided about allocation concealment and there- } \\
\text { fore it was unclear if allocation was concealed. }\end{array}$ \\
\hline $\begin{array}{l}\text { Blinding of participants } \\
\text { and personnel (perfor- } \\
\text { mance bias) }\end{array}$ & High risk & $\begin{array}{l}\text { Environment and Policy Assessment and Observation (EPAO). Although the as- } \\
\text { sessor was blinded, there was no mention that the participants were blinded } \\
\text { and therefore there was a high risk of performance bias. }\end{array}$ \\
\hline $\begin{array}{l}\text { Blinding of outcome as- } \\
\text { sessment (detection bias) } \\
\text { All outcomes }\end{array}$ & Low risk & $\begin{array}{l}\text { Environment and Policy Assessment and Observation (EPAO). The EPAOs were } \\
\text { completed by graduate student interns who were blinded to the study arm of } \\
\text { the classrooms being observed. }\end{array}$ \\
\hline $\begin{array}{l}\text { Incomplete outcome data } \\
\text { (attrition bias) } \\
\text { All outcomes }\end{array}$ & Unclear risk & $\begin{array}{l}\text { There was no attrition for the EPAOs (this was completed for all 23 class- } \\
\text { rooms). }\end{array}$ \\
\hline $\begin{array}{l}\text { Selective reporting (re- } \\
\text { porting bias) }\end{array}$ & Unclear risk & $\begin{array}{l}\text { The Wilkin protocol paper was for the main study and the research reported } \\
\text { by Equivel was embedded within it. The Wilkin protocol paper may not have } \\
\text { intended to report the outcomes for the embedded research and therefore it } \\
\text { was unclear whether there was selective outcome reporting. }\end{array}$ \\
\hline
\end{tabular}

Finch 2012

\begin{tabular}{ll}
\hline Methods & Study design: nonrandomised trial \\
Intervention duration: 3 months (staggered) \\
Length of follow-up from baseline: 18 months (follow-up was conducted approximately 12 months \\
after the initiation of the intervention with wave 1 services, and approximately 6 months after the initi- \\
ation of the intervention for wave 2 services) \\
Differences in baseline characteristics: reported \\
Unit of allocation: childcare service \\
Unit of analysis: childcare service \\
Service type: long daycare services and preschools \\
Region: Intervention: Hunter New England region, New South Wales, Australia; Control: New South \\
Wales, Australia \\
Demographic/socioeconomic characteristics: Intervention: the intervention region included a large \\
non-metropolitan area (more than 130,000 km 2 ) encompassing urban and rural communities with a \\
population of 60,970 children aged 0 to 5 years. Control: the comparison region of New South Wales \\
had an area of 801,305 km² and included major cities, inner regional services, outer regional services, \\
remote and very remote areas. New South Wales has a population of 506,095 children aged 0 to 5 years. \\
Inclusion/exclusion criteria: inclusion criteria: long daycare services and preschools in the Hunter \\
New England area (intervention group) or the remainder of New South Wales (comparison group) as
\end{tabular}

Strategies to improve the implementation of healthy eating, physical activity and obesity prevention policies, practices or programmes 
Finch 2012 (Continued)

recorded by the licensing agency for such services. Exclusion criteria: services catering solely for children with special needs such as intellectual or physical disabilities

Number of services randomised: 484 services participated in baseline measures. Intervention: 275 (not randomised - those services approached who agreed to participate and completed baseline data collection). Control: 209 (of those randomly approached and who took part in baseline evaluation).

Numbers by trial group:

$\mathrm{n}$ (control baseline $)=209$

$\mathrm{n}$ (control follow-up) $=164$

$\mathrm{n}$ (intervention baseline) $=275$

$\mathrm{n}$ (intervention follow-up) $=228$

Recruitment: Intervention: all services $(n=338)$ located within the intervention region were invited to participate. Control: a simple random sample of eligible centre-based childcare services in all other regions of the state of New South Wales were invited to participate in the study as the comparison group $(n=298)$.

Recruitment rate: Intervention: 81\%; Control: $83 \%$

Interventions

Number of experimental conditions: 2 (intervention, control)

Policies, practices or programmes targeted by the intervention:

Physical activity policy:

- Conducting daily fundamental movement sessions with recommended components

- Time spent on structured physical activities

- All staff usually participate in free active play

- All staff usually provide verbal prompts for physical activity

- Children are allowed to watch small screen recreation less than once per week

- Children participate in seated activities for no longer than 30 minutes at a time

- Staff trained in physical activity

Implementation strategies:

- Offer of staff training (1 x 6-hour workshop)

- Offer of information programme resources and instructional materials

- Offer of follow-up support ( 2 × 15-minute support calls, 2 support emails/faxes, 6 project newsletters)

- Provision of performance monitoring and feedback regarding policy and practice adoption

- Offer of incentives

Who delivered the intervention: the staff training was delivered by external experts and follow-up support and performance monitoring and feedback (telephone) was delivered by health service staff.

Theoretical underpinning: not reported

Description of control:

- Childcare service staff were invited to attend a full day workshop provided by a non-government organisation.

- Provision of a printed resource folder

- Provision of a small financial grant to support staff attendance at training or the purchase of equipment

- Opportunity for additional support strategies to be provided by local health services at their discretion

Outcomes

Outcome relating to the implementation of childcare service policies, practices or programmes: Services with a physical activity policy that referred to:

- Child fundamental movement skills development

- Limits on small screen recreation and TV

- Physical activity training for staff

- Services conducting daily fundamental movement sessions with recommended components

- Time spent on structured physical activities

- Services where all staff usually participate in free active play (role modelling)

- Services where all staff usually provide verbal prompts for physical activity

- Services where children are allowed to watch small screen recreation less than once per week

- Services where children participate in seated activities for no longer than 30 minutes at a time

- Services with staff trained in physical activity

Data collection method: service manager self-report via computer-assisted telephone interview

Validity of measures used: unclear (developed following review of existing validated tools and pretested prior to use)

Outcome relating to cost: not applicable

Strategies to improve the implementation of healthy eating, physical activity and obesity prevention policies, practices or programmes 
Finch 2012 (Continued)

Outcome relating to adverse consequences: not applicable

Outcome relating to child diet, physical activity or weight status: not applicable

Outcome relating to implementation strategy acceptability, adoption, penetration, sustainability and appropriateness:

\section{Acceptability}

Data collection method: telephone interview conducted with childcare service managers

Validity of measures used: not reported

\section{Penetration}

Data collection method: not reported

Validity of measures used: not reported

The study had multiple outcomes but did not appear to adjust the P value for multiple comparisons.
This work was supported by funding received from the NSW Ministry of Health ASSIST programme. The
project also received infrastructure support from the Hunter Medical Research Institute (HMRI) and
Hunter New England Population Health.

\section{Risk of bias}

\begin{tabular}{lll}
\hline Bias & Authors' judgement & Support for judgement \\
\hline $\begin{array}{ll}\text { Random sequence genera- } \\
\text { tion (selection bias) }\end{array}$ & High risk & $\begin{array}{l}\text { Nonrandomised experimental design. High risk of selection bias as the inter- } \\
\text { vention services were recruited from a selected area. Control services were } \\
\text { randomly selected from a comparison region. No detail was provided regard- } \\
\text { ing the sequence generation procedure used to randomise control services for } \\
\text { selection. Table } 2 \text { shows that services within the intervention and comparison } \\
\text { sites differed significantly in terms of socioeconomic areas, geographic locality } \\
\text { and services with children of an Aboriginal background. }\end{array}$ \\
\end{tabular}

\begin{tabular}{|c|c|c|}
\hline $\begin{array}{l}\text { Allocation concealment } \\
\text { (selection bias) }\end{array}$ & High risk & $\begin{array}{l}\text { Nonrandomised experimental design. Intervention services were recruited } \\
\text { from a selected area, therefore high risk of bias as there was no concealment } \\
\text { of allocation. }\end{array}$ \\
\hline
\end{tabular}

\begin{tabular}{ll}
\hline $\begin{array}{l}\text { Blinding of participants } \\
\text { and personnel (perfor- }\end{array}$ & Unclear risk \\
$\begin{array}{l}\text { mance bias) } \\
\text { All outcomes }\end{array}$ & $\begin{array}{l}\text { We assumed that due to the nature of the intervention, childcare service staff } \\
\text { and study personnel delivering the intervention were not blind to the study al- } \\
\text { location, however, as the control group may have also received some form of } \\
\text { intervention, systematic bias between groups in terms of performance bias } \\
\text { was unknown. }\end{array}$
\end{tabular}

\begin{tabular}{|c|c|c|}
\hline $\begin{array}{l}\text { Blinding of outcome as- } \\
\text { sessment (detection bias) } \\
\text { All outcomes }\end{array}$ & High risk & $\begin{array}{l}\text { Self-reported physical activity policies and practices. No blinding of research } \\
\text { personnel } \\
\text { or participants (service managers) and due to the self-report of this outcome, } \\
\text { the } \\
\text { risk of bias was considered to be high. }\end{array}$ \\
\hline
\end{tabular}

\begin{tabular}{|c|c|c|}
\hline $\begin{array}{l}\text { Incomplete outcome data } \\
\text { (attrition bias) } \\
\text { All outcomes }\end{array}$ & Unclear risk & $\begin{array}{l}83 \% \text { of intervention group services included in final post-test data analysis; } \\
78 \% \text { of comparison group services included in final post-test data analysis. } \\
\text { While these proportions were similar, it was unclear whether the services lost } \\
\text { to follow-up differed between groups. No sensitivity analysis reported to test } \\
\text { assumptions regarding missing data }\end{array}$ \\
\hline
\end{tabular}

\begin{tabular}{ll}
\hline $\begin{array}{l}\text { Selective reporting (re- } \\
\text { porting bias) }\end{array}$ & $\begin{array}{l}\text { No prospective trial protocol or trial registration so it was unclear whether } \\
\text { there was selective outcome reporting. }\end{array}$
\end{tabular}


Finch 2012 (Continued)

Potential confounding Unclear risk Authors stated that "Characteristics of services were not adjusted for in the logistic regression model as we were looking at change within services and the baseline score of the services effectively controlled for potential differences in baseline characteristics between the two regions." It is unknown whether this was adequate to reduce bias due to known confounders.

Study design: Cluster-RCT
Intervention duration: 7 months
Length of follow-up from baseline: 8 months
Differences in baseline characteristics: reported
Unit of allocation: childcare service
Unit of analysis: childcare service (child physical activity was assessed at the level of the individual)

Participants Service type: centre-based long daycare services

Region: Hunter region, New South Wales

Demographic/socioeconomic characteristics: the Hunter region encompasses nonmetropolitan 'major cities' and 'inner regional' areas with 14,061 children aged 3 to 5 years residing in the area. $5 \%$ of residents speak languages other than English and $2 \%$ of residents are of Aboriginal or Torres Strait Islander origin. The Hunter region has lower indices of socioeconomic status than the New South Wales state average.

Inclusion/exclusion criteria: inclusion criteria: centre-based long daycare services. Services were required to have at least 25 enrolled children aged between 3 to 5 years. Children aged 3 to 5 years attending participating services were eligible for the study if they attended on the day of the week nominated by the service manager for baseline data collection.

Number of services randomised: 20 services ( 10 intervention ( 242 children), 10 control ( 215 children) Numbers by trial group:

$\mathrm{n}$ (controls baseline $)=10$ services

$\mathrm{n}$ (controls follow-up) $=10$ services

$\mathrm{n}$ (interventions baseline) $=10$ services

$\mathrm{n}$ (interventions follow-up) $=10$ services

Recruitment: a total of 70 childcare services in the study region served as the sampling frame. Recruitment rate: $54 \%$

Number of experimental conditions: 2 (intervention, control)

Policies, practices or programmes targeted by the intervention:

- Fundamental movement skill development activity sessions

- Staff delivery of structured physical activity

- Staff role modelling of active play and delivery of verbal prompts

- Limiting small screen recreation and sedentary time

- Providing children with a physical activity-promoting indoor and outdoor physical environment

- Physical activity policy

Implementation strategies:

- Staff training (6-hour workshop for childcare service staff)

- Provision of resources

- Follow-up support (2 telephone support calls and a 2-hour service visit)

- Performance feedback via project newsletter on 2 occasions

- Incentives

- Opinion leaders

Who delivered the intervention: workshop and follow-up component delivered by experts

Theoretical underpinning: the multi-level intervention was designed using the social ecological models of health behaviour change.

Description of control: waiting-list control group that did not receive the intervention or any intervention support or materials during the study period and were offered the intervention after collection of all follow-up data 
Finch 2014 (Continued)

Outcomes

Outcome relating to the implementation of childcare service policies, practices or programmes:

- Fundamental movement skill development activity sessions

- Staff delivery of structured physical activity

- Staff role modelling of active play and delivery of verbal prompts

- Limiting small screen recreation and sedentary time

- Physical activity-promoting resources and materials

- Portable equipment

- Physical activity policy

Data collection method: observational audit - EPAO was conducted by 2 trained research staff

Validity of measures used: unclear - EPAO has reported high inter-observer agreement but other psychometric properties of this tool were not reported

Outcome relating to cost: not applicable

Outcome relating to adverse consequences:

The number of child injuries recorded at the service in the month of data collection at baseline and follow-up

Data collection method: service manager self-report via interview

Validity of measures used: unclear

Outcome relating to child diet, physical activity or weight status:

Child step count:

Data collection method: pedometer worn for 1 day during attendance at the childcare service

Validity of measures used: a valid measure of physical activity in preschool age children

Outcome relating to implementation strategy acceptability, adoption, penetration, sustainability and appropriateness:

\section{Acceptability}

Data collection method: written survey conducted with childcare service managers

Validity of measures used: not reported

\section{Penetration}

Data collection method: Programme records collected by the research team during implementation Validity of measures used: not reported

This work was supported by funding received from the NSW Ministry of Health ASSIST programme and the Hunter Medical Research Institute (HMRI).

\section{Risk of bias}

\begin{tabular}{lll}
\hline Bias & Authors' judgement & Support for judgement \\
\hline $\begin{array}{l}\text { Random sequence genera- } \\
\text { tion (selection bias) }\end{array}$ & Low risk & $\begin{array}{l}\text { Computerised random number function in Microsoft Excel was used to gener- } \\
\text { ate random number sequence. }\end{array}$ \\
\hline $\begin{array}{l}\text { Allocation concealment } \\
\text { (selection bias) }\end{array}$ & Low risk & $\begin{array}{l}\text { Statistician not involved in the project allocated the services to groups using a } \\
\text { computerised programme. }\end{array}$ \\
\hline $\begin{array}{l}\text { Blinding of participants } \\
\text { and personnel (perfor- } \\
\text { mance bias) }\end{array}$ & High risk & $\begin{array}{l}\text { We assumed that, due to the nature of the intervention, childcare service staff } \\
\text { and study personnel delivering the intervention were not blind to the study al- } \\
\text { location and therefore there was a potential high risk of performance bias. }\end{array}$ \\
\hline $\begin{array}{l}\text { Blinding of outcome as- } \\
\text { sessment (detection bias) }\end{array}$ & Low risk & $\begin{array}{l}\text { Implementation of policies and practices measured using observational audit } \\
\text { - research }\end{array}$
\end{tabular}

Strategies to improve the implementation of healthy eating, physical activity and obesity prevention policies, practices or programmes 49 within childcare services (Review)

Copyright $\odot 2020$ The Cochrane Collaboration. Published by John Wiley \& Sons, Ltd. 
Finch 2014 (Continued)

All outcomes
Incomplete outcome data Low risk (attrition bias)

All outcomes staff undertaking audits were blind to group allocation.

Implementation of policies and practices - no loss to follow-up (10 intervention services; 10 control services)

\begin{tabular}{ll}
\hline $\begin{array}{l}\text { Selective reporting (re- } \\
\text { porting bias) }\end{array}$ & Low risk \\
\end{tabular}

\begin{tabular}{lll}
\hline Recruitment to cluster & Low risk & $\begin{array}{l}\text { For the child physical activity measure, children were recruited by service } \\
\text { managers at the service selecting a day of the week for measurement to occur. } \\
\text { Allocation was not revealed to services until after baseline data collection. }\end{array}$ \\
\hline Baseline imbalance & High risk & $\begin{array}{l}\text { Baseline imbalance in services in areas of higher socioeconomic status (in- } \\
\text { tervention } 90 \%, \text { control } 60 \% \text { ) and average years of operation (intervention } 8 \\
\text { years, control } 20 \text { years) and no mention of adjustment within analysis }\end{array}$ \\
\hline Loss of clusters & Low risk & $100 \%$ followed up \\
\hline $\begin{array}{l}\text { Incorrect analysis } \\
\text { Compatibility with individ- } \\
\text { ually randomised RCTs }\end{array}$ & Lnclear risk & Generalised linear mixed model accounting for children nested within services \\
\hline
\end{tabular}

Finch 2019

Study design: Cluster-RCT
Intervention duration: 10 months
Length of follow-up from baseline: 12 months
Differences in baseline characteristics: reported
Unit of allocation: childcare service
Unit of analysis: childcare service

Region: Hunter New England region of NSW, Australia

Demographic/socioeconomic characteristics: The Hunter New England Region encompasses nonmetropolitan 'major cities', 'inner regional', 'outer regional' and 'remote' areas, as described by the Australian Standard Geographic Classification system. The Hunter New England Region has lower indices of socioeconomic status than the NSW state average.

Inclusion/exclusion criteria: Services catering exclusively for children requiring specialist care, mobile preschools, and Department of Education and Communities preschools were excluded, as were services already involved in an alternative RCT currently underway in the Hunter New England region. Services already identified through local health service data as comprehensively implementing healthy eating and physical activity policies and practices were also excluded.

\section{Numbers by trial group: \\ $\mathrm{n}$ (controls baseline $)=46$ \\ $n$ (controls follow-up) $=43$}

Number of services randomised: 131 
Finch 2019 (Continued)

$$
\begin{aligned}
& n(\text { interventions baseline })=62 \\
& n(\text { interventions follow-up })=57
\end{aligned}
$$

\section{Recruitment:}

Service: Of the 366 childcare services in the region, 128 were excluded given their involvement in an alternative RCT, a further 30 did not meet inclusion criteria, and an additional 77 were identified as comprehensively implementing healthy eating and physical activity policies and practices. A total of 131 services were randomised, among which 68 were allocated to the intervention and 63 to the control. Six services allocated to the intervention and 17 services allocated to the control group did not provide baseline data and were therefore excluded.

\section{Recruitment rate: $82 \%$}

\section{Policies, practices or programmes targeted by the intervention:}

- Service having written nutrition, physical activity, and small screen recreation policies

- Service providing information to families

- Service providing structured and specific learning experiences about healthy eating at least two times per week

- Service supplying age-appropriate drinks to children

- Service conducting fundamental movement skills activities for children aged 3-5 years every day to at least $90 \%$ of children

- Service limiting the use of small screen recreation by children aged 3-5 years to only educational purposes and for learning experiences

\section{Implementation strategies:}

Educational materials: Intervention services were provided with resources to support the implementation of these policies (policy templates, DVD, manuals, posters, and parent lunchbox resources).

Audit with feedback: Facilitated performance feedback was provided to services regarding implementation of targeted policies and practices.

Continuous quality improvement: processes including review of progress, positive reinforcement, and discussion of deficits identified from feedback reports, problem-solving, goal setting, and action planning were incorporated.

Educational outreach or academic detailing: The initial performance review was completed in-person by trained support officers.

Opinion leaders: Nominated supervisors were expected to endorse implementation of the targeted practices and to communicate goals and action plans, as well as progress to service staff.

Tailored interventions: Where services were already meeting a policy or practice, implementation support was directed towards policies and practices not yet achieved.

Who delivered the intervention: Local Health District Support Officers

Theoretical underpinning: Consolidated Framework for Implementation Research

Description of control: Control group services received the same four electronic newsletters during the intervention period, but did not receive any other resources. At completion of the intervention period, control services were offered the intervention. 
Finch 2019 (Continued)

Change in prevalence of services implementing all six targeted healthy eating and physical activity policies and practices at 12 months

Data collection method: telephone interview with nominated supervisor or lead educator to determine mean number policies and practices and proportion of services implementing practices

Validity of measures used: not reported

Outcome relating to cost: not applicable

Outcome relating to adverse consequences: not applicable

Outcome relating to child diet, physical activity or weight status: not applicable

Outcome relating to implementation strategy acceptability, adoption, penetration, sustainability and appropriateness:

\section{Acceptability}

Data collection method: Computer-assisted telephone interview with nominated supervisor or lead educator to determine service satisfaction with the intervention components. Eleven items were assessed using a 7-point Likert scale ( 1 = strongly disagree to 7 = strongly agree).

Validity of measures used: not reported

Notes

Infrastructure funding for the study was provided in kind by Hunter New England Population Health, together with funding from the Hunter Medical Research Institute, University of Newcastle Priority Research Centre for Health Behaviour and Cancer Council NSW (grant ID: PG 16-05). Associate Professor Luke Wolfenden receives salary support as a Hunter New England Clinical Research Fellow and is supported by Heart Foundation Future Leader Fellowship (Award No. 101175) and an NHMRC Career Development Fellowship (APP1128348). Dr Alice Grady receives salary support from a NHMRC grant (grant ID: APP1102943). Dr Sze Lin Yoong is a postdoctoral research fellow funded by the National Heart Foundation (Award No. 100547) and Australian Research Council (DE170100382).

\section{Risk of bias}

\begin{tabular}{lll}
\hline Bias & Authors' judgement & Support for judgement \\
\hline $\begin{array}{l}\text { Random sequence genera- } \\
\text { tion (selection bias) }\end{array}$ & Unclear risk & $\begin{array}{l}\text { While a computerised random number generator was used to randomise } \\
\text { childcare services into treatment groups, following randomisation } 6 \text { of } 68\end{array}$ \\
& $\begin{array}{l}(8.8 \%) \text { intervention services and } 17 \text { of } 63(28 \%) \text { control services were removed } \\
\text { because they did not provide baseline data. It was unclear whether, follow- } \\
\text { ing this removal of services, the groups remained similar at baseline as was in- } \\
\text { tended by the randomisation. }\end{array}$
\end{tabular}

\begin{tabular}{lll}
\hline $\begin{array}{l}\text { Allocation concealment } \\
\text { (selection bias) }\end{array}$ & Low risk & All allocation undertaken at one time by a computer system. \\
\hline $\begin{array}{l}\text { Blinding of participants } \\
\text { and personnel (perfor- } \\
\text { mance bias) } \\
\text { All outcomes }\end{array}$ & High risk & $\begin{array}{l}\text { Childcare services were not blinded to group allocation and therefore there } \\
\text { was a high risk of performance bias. }\end{array}$ \\
\hline $\begin{array}{l}\text { Blinding of outcome as- } \\
\text { sessment (detection bias) } \\
\text { All outcomes }\end{array}$ & High risk & $\begin{array}{l}\text { The nominated supervisor completed the computer-assisted telephone inter- } \\
\text { view and was not blinded to condition and therefore the risk of detection bias } \\
\text { was high. }\end{array}$ \\
\hline $\begin{array}{l}\text { Incomplete outcome data } \\
\text { (attrition bias) } \\
\text { All outcomes }\end{array}$ & Unclear risk & $\begin{array}{l}\text { Overall, 11/68 (17.7\%) services randomised to the control group had missing } \\
\text { follow-up data; and } 20 / 63(31.7 \%) \text { services allocated to the control group had } \\
\text { missing follow-up data. }\end{array}$ \\
\hline
\end{tabular}


Finch 2019 (Continued)

Selective reporting (reporting bias)

Unclear risk
The primary outcome aligned with that reported in the protocol paper. However, other measures were not prospectively registered, such as the proportion of services that implemented each of the policies and practices and the mean number of practice services that were compliant.

Gosliner 2010

$\begin{array}{ll}\text { Methods } & \text { Study design: RCT } \\ \text { Intervention duration: not reported } \\ \text { Length of follow-up from baseline: } 10 \text { months } \\ \text { Differences in baseline characteristics: not reported by group } \\ \text { Unit of allocation: childcare service } \\ \text { Unit of analysis: childcare service staff }\end{array}$

Participants

Service type: childcare centres

Region: California, USA

Demographic/socioeconomic characteristics: childcare services were located in low-income neighbourhoods in Northern California.

Inclusion/exclusion criteria: inclusion criteria: services that were already participating in a health education and policy development project (Child Health and Nutrition Service Enhancement) with the Contra Costa Child Care Council

Number of services randomised: 18 (9 intervention, 9 control)

Numbers by trial group:

$\mathrm{n}$ (controls baseline) $=7$

$\mathrm{n}$ (controls follow-up) $=7$

$\mathrm{n}$ (interventions baseline) $=6$

$\mathrm{n}$ (interventions follow-up) $=6$

Recruitment: 9 pairs of eligible services were matched on city of location and programme size and were randomised to either the intervention or control group.

Recruitment rate: $84 \%$ entered the study.

\title{
Interventions
}

\author{
Number of experimental conditions: 2 (intervention, control) \\ Policies, practices or programmes targeted by the intervention: \\ Nutrition and physical activity policies, children's food and physical activity environment \\ Implementation strategies: \\ - Training and technical assistance regarding children's health and nutrition \\ - Received a set of nutrition and physical activity policies \\ - Staff wellness programme consisting of: \\ - Kick-off wellness training with individual health consultations including education, individual health \\ assessments \\ - Monthly newsletters and information with pay-checks promoting healthy eating and physical activity \\ - Group walking programme with awards for reaching milestones \\ - Staff follow-up support visits \\ Theoretical underpinning: not reported \\ Description of control: received training and technical assistance regarding children's health and nu- \\ trition and received a set of nutrition and physical activity policies
Outcome relating to the implementation of childcare service policies, practices or programmes:
- Staff providing fresh fruits in children's meals and snacks more often during the past year
- Staff providing fresh vegetables in children's meals and snacks more often during the past year
- Staff providing sweetened beverages in children's meals and snacks more often during the past year
- Staff providing sweetened foods in children's meals and snacks more often during the past year
- Staff providing fresh fruits in children's celebrations more often during the past year
- Staff providing fresh vegetables in children's celebrations more often during the past year
- Staff providing sweetened beverages in children's celebrations more often during the past year
- Staff providing sweetened foods in children's celebrations more often during the past year

Outcomes 
Gosliner 2010 (Continued)

Data collection method: childcare service staff self-report via questionnaire

Validity of measures used: unclear

Outcome relating to cost: not applicable

Outcome relating to adverse consequences: not applicable

Outcome relating to child diet, physical activity or weight status: not applicable

Outcome relating to implementation strategy acceptability, adoption, penetration, sustainability and appropriateness:

\section{Penetration}

Data collection method: self-administered questionnaires with staff at participating childcare services Validity of measures used: not reported

Notes The study did not report baseline values for the implementation outcomes..

This study was supported by the Food Nutrition Education programme of the USA Department of Agriculture.

\section{Risk of bias}

\begin{tabular}{lll}
\hline Bias & Authors' judgement & Support for judgement \\
\hline $\begin{array}{l}\text { Random sequence genera- } \\
\text { tion (selection bias) }\end{array}$ & Unclear risk & $\begin{array}{l}\text { Services were matched on city of location and programme size and were ran- } \\
\text { domised to intervention or control group. The sequence generation procedure } \\
\text { was not reported. }\end{array}$ \\
\hline
\end{tabular}

\begin{tabular}{ll}
\hline $\begin{array}{l}\text { Allocation concealment } \\
\text { (selection bias) }\end{array}$ & Unclear risk
\end{tabular} $\begin{aligned} & \text { Whether pending allocation was concealed was unclear as no information was } \\
& \text { provided on concealment. }\end{aligned}$

\begin{tabular}{|c|c|c|}
\hline $\begin{array}{l}\text { Blinding of participants } \\
\text { and personnel (perfor- } \\
\text { mance bias) } \\
\text { All outcomes }\end{array}$ & High risk & $\begin{array}{l}\text { We assumed that, due to the nature of the intervention, childcare service staff } \\
\text { and study personnel delivering the intervention were not blind to the study al- } \\
\text { location and therefore there was a potential high risk of performance bias. }\end{array}$ \\
\hline
\end{tabular}

\begin{tabular}{|c|c|c|}
\hline $\begin{array}{l}\text { Blinding of outcome as- } \\
\text { sessment (detection bias) } \\
\text { All outcomes }\end{array}$ & High risk & $\begin{array}{l}\text { Self-reported by service managers, therefore high risk of bias due to managers } \\
\text { being } \\
\text { aware of allocation }\end{array}$ \\
\hline
\end{tabular}

\begin{tabular}{|c|c|c|}
\hline $\begin{array}{l}\text { Incomplete outcome data } \\
\text { (attrition bias) } \\
\text { All outcomes }\end{array}$ & High risk & $\begin{array}{l}\text { Data were available for } 50(56 \%) \text { participants in the intervention group and } \\
39(44 \%) \text { in the control group. Of those not returning at end point, most had } \\
\text { changed employment }\end{array}$ \\
\hline
\end{tabular}

$(80 \%)$ or were on leave or vacation $(14 \%)$.

7 intervention staff who reported participating in fewer than half of the intervention activities were considered inadequately exposed and were excluded from the analysis, leaving 43 staff in the intervention group. Therefore, the intention-to-treat principle was not applied.

\begin{tabular}{|c|c|c|}
\hline $\begin{array}{l}\text { Selective reporting (re- } \\
\text { porting bias) }\end{array}$ & Unclear risk & $\begin{array}{l}\text { No prospective trial protocol or trial registration so it was unclear whether } \\
\text { there was selective outcome reporting }\end{array}$ \\
\hline
\end{tabular}

Hardy 2010

\begin{tabular}{ll}
\hline Methods & Study design: Cluster-RCT \\
& Intervention duration: 5 months \\
& Length of follow-up from baseline: 5 months \\
\hline
\end{tabular}

Strategies to improve the implementation of healthy eating, physical activity and obesity prevention policies, practices or programmes 
Hardy 2010 (Continued)

Differences in baseline characteristics: reported

Unit of allocation: childcare service

Unit of analysis: childcare service

Service type: preschools
Region: Sydney, New South Wales, Australia
Demographic/socioeconomic characteristics: not described
Inclusion/exclusion criteria: inclusion criteria: preschools operating under the aus
South Wales Department of Education and Training located in the Sydney, Western
Western Sydney education regions of New South Wales
Number of services randomised: 29 (15 intervention, 14 control)
Numbers by trial group:
$\mathrm{n}$ (controls baseline) $=14$
$\mathrm{n}$ (controls follow-up) 14
$\mathrm{n}$ (interventions baseline) $=15$
$\mathrm{n}$ (interventions follow-up) $=15$
Recruitment: all eligible preschools were invited to participate in the study $(\mathrm{n}=61)$
Recruitment rate: $48 \%$

Interventions

Number of experimental conditions: 2 (intervention, control)

Policies, practices or programmes targeted by the intervention:

'Munch and Move' programme:

- Healthy eating and ways of incorporating food-based activities into the education programme

- Physical activity and ways of incorporating fun, games-based skills activities into the programme

- Strategies to encourage children to limit their recreational screen time

- Providing opportunities for children to engage in unstructured physically active play

- Developing and implementing healthy nutrition and physical activity fundraising policies Implementation strategies:

- 1-day professional development workshop for up to 2 staff, delivered by a specialised early childhood training organisation

- Resources for preschools that included a manual and a small grant to support staff to attend training or purchase physical activity equipment for the service

- Contact with health promotion professionals from the local health service, to provide additional advice to preschools to support the delivery of the programme including 2 visits post-workshop

Who delivered the intervention: experts and health service staff

Theoretical underpinning: not reported

Description of control: control preschools received health information on unrelated topics (road safety and sun safety) during the intervention period.

Outcomes

Outcome relating to the implementation of childcare service policies, practices or programmes:

- Structured play time (minutes per session)

- Frequency of structured play (sessions per week)

- Unstructured play time (minutes per session)

- Frequency of unstructured play (sessions per week)

- Fundamental movement skill activities (minutes per session)

- Frequency of fundamental movement skill activities (sessions per week)

- Conduct of food-based activities

- Rules concerning food and drink brought in from home

- Food policies

- Communicating food rules and policies to parents

Data collection method: interview with the service manager

Validity of measures used: unclear

Outcome relating to cost: not applicable

Outcome relating to adverse consequences: not applicable

Outcome relating to child diet, physical activity or weight status: not applicable

Outcome relating to implementation strategy acceptability, adoption, penetration, sustainability and appropriateness:

\section{Penetration}

Strategies to improve the implementation of healthy eating, physical activity and obesity prevention policies, practices or programmes 
Hardy 2010 (Continued)

Data collection method: not reported

Validity of measures used: not reported

\section{Acceptability}

Data collection method: self-report questionnaires with childcare service staff

Validity of measures used: not reported

\section{Notes}

The Munch and Move programme and this evaluation study were funded by Centre for Health Advancement, NSW Department of Health.

\section{Risk of bias}

\begin{tabular}{|c|c|c|}
\hline Bias & Authors' judgement & Support for judgement \\
\hline $\begin{array}{l}\text { Random sequence genera- } \\
\text { tion (selection bias) }\end{array}$ & Unclear risk & The procedure for random sequence generation was not described. \\
\hline $\begin{array}{l}\text { Allocation concealment } \\
\text { (selection bias) }\end{array}$ & Unclear risk & $\begin{array}{l}\text { Whether pending allocation was concealed was unclear as no information was } \\
\text { provided on concealment. }\end{array}$ \\
\hline $\begin{array}{l}\text { Blinding of participants } \\
\text { and personnel (perfor- } \\
\text { mance bias) } \\
\text { All outcomes }\end{array}$ & High risk & $\begin{array}{l}\text { We assumed that, due to the nature of the intervention, childcare service staff } \\
\text { and study personnel delivering the intervention were not blind to the study al- } \\
\text { location and therefore there was a potential high risk of performance bias. }\end{array}$ \\
\hline $\begin{array}{l}\text { Blinding of outcome as- } \\
\text { sessment (detection bias) } \\
\text { All outcomes }\end{array}$ & High risk & $\begin{array}{l}\text { Policies and practices - self-reported by service managers in interviews with } \\
\text { research } \\
\text { staff, therefore high risk of bias due to managers being aware of allocation }\end{array}$ \\
\hline $\begin{array}{l}\text { Incomplete outcome data } \\
\text { (attrition bias) } \\
\text { All outcomes }\end{array}$ & Low risk & $\begin{array}{l}\text { All service managers followed up in both groups - therefore, low risk of bias for } \\
\text { outcome regarding implementation of policies and practices }\end{array}$ \\
\hline $\begin{array}{l}\text { Selective reporting (re- } \\
\text { porting bias) }\end{array}$ & Unclear risk & $\begin{array}{l}\text { No prospective trial protocol or trial registration so it was unclear whether } \\
\text { there was selective outcome reporting. }\end{array}$ \\
\hline Recruitment to cluster & Low risk & $\begin{array}{l}\text { All parents of participating services were invited to allow their children to par- } \\
\text { ticipate. }\end{array}$ \\
\hline Baseline imbalance & Unclear risk & $\begin{array}{l}\text { Unclear response rate of children in each group - imbalance in numbers of stu- } \\
\text { dents (intervention 263, control 167); some imbalances in baseline character- } \\
\text { istics (mean years teaching experience: intervention } 4.5 \text { years, control } 6 \text { years; } \\
\text { teacher's aide: intervention } 11.1 \text { years, control } 8.9 \text { years; children attending } 2 \\
\text { days per week: intervention } 22 \% \text {, control } 11 \% \text {; children attending } 3 \text { days per } \\
\text { week: intervention } 21 \% \text {, control } 42 \% \text {; English speaking: intervention } 58 \% \text {, con- } \\
\text { trol } 41 \% \text { - unknown if any were significantly different. Adjustment of some } \\
\text { characteristics in analysis }\end{array}$ \\
\hline Loss of clusters & Low risk & No loss of clusters \\
\hline Incorrect analysis & Low risk & CSPlan procedure used to allow for clustering within service class \\
\hline $\begin{array}{l}\text { Compatibility with individ- } \\
\text { ually randomised RCTs }\end{array}$ & Unclear risk & Unable to determine if a herd effect existed \\
\hline
\end{tabular}




$\begin{array}{ll}\text { Methods } & \text { Study design: parallel-group RCT } \\ \text { Intervention duration: not specified } \\ \text { Length of follow-up from baseline: not specified } \\ \text { Differences in baseline characteristics: not reported } \\ \text { Unit of allocation: childcare service } \\ \text { Unit of analysis: childcare service } \\ \text { Service type: preschools } \\ \text { Region: Republic of Ireland } \\ \text { Demographic/socioeconomic characteristics: preschools were situated in towns, villages and the } \\ \text { countryside across 4 Midland counties in a geographical area defined as disadvantaged. } \\ \text { Inclusion/exclusion criteria: inclusion criteria: preschools providing a "full day care service". Exclu- } \\ \text { sion criteria: preschools that provided only sessional or part-time care for children; preschools desig- } \\ \text { nated as ineligible by the Preschool Inspection Team due to insufficient standard in other predefined } \\ \text { areas of inspection; preschools that had not been inspected by the Preschool Inspection Team in the } \\ \text { previous } 12 \text {-month period } \\ \text { Number of services randomised: } 61 \text { (30 intervention group 'manager trained', } 31 \text { intervention group } \\ \text { 'manager and staff trained') } \\ \text { Numbers by trial group: } \\ \mathrm{n} \text { (intervention group 'manager trained' baseline) = 30 } \\ \mathrm{n} \text { (intervention group 'manager trained' follow-up) = 24 } \\ \mathrm{n} \text { (intervention group 'manager and staff trained' baseline) = 31 } \\ \mathrm{n} \text { (intervention group 'manager and staff trained' follow-up) = 18 } \\ \text { Recruitment: convenience sampling was undertaken. An up-to-date list of preschools ( } \mathrm{n}=100) \text { provid- } \\ \text { ing a 'full daycare service' was obtained and these preschools were invited to participate. } \\ \text { Recruitment rate: } 61 \%\end{array}$

Interventions Number of experimental conditions: 2 (intervention group 'manager trained', intervention group 'manager and staff trained')

Policies, practices or programmes targeted by the intervention:

- Adequate meal and snack composition

- Healthy foods and fluids

- Appropriate serving size provision

- Family-style food service

- Healthy preschool policy development

Implementation strategies: Intervention 'manager trained'

- 1-hour manager training session with a research dietitian

- Provision of resources and best practice criterion

- Provision of individualised 'written feedback record' from a pre-intervention observation visit and suggested strategies for improvement discussed with the manager

Implementation strategies: Intervention 'manager and staff trained'

- 1-hour manager training session with a research dietitian

- 1.5-hour structured staff education session with a research dietitian including presentation, group work exercises and discussion

- Provision of resources and best practice criterion

- Provision of individualised 'written feedback record' from a pre-intervention observation visit and suggested strategies for improvement discussed with the manager and staff

Who delivered the intervention: dietitians

Theoretical underpinning: adult learning methodologies

Outcomes

Outcome relating to the implementation of childcare service policies, practices or programmes:

- Environment

- Food service

- Meals

- Snacks

- Overall score

Data collection methods: 1 day observation, preschool manager self-report 
Johnston Molloy 2013 (Continued)

Validity of measures used: used the validated Preschool Health Promotion Activity Scored Evaluation

Form

Outcome relating to cost: not applicable

Outcome relating to adverse consequences: not applicable

Outcome relating to child diet, physical activity or weight status: not applicable

Outcome relating to implementation strategy acceptability, adoption, penetration, sustainability and appropriateness:

\section{Penetration}

Data collection method: postal survey completed by childcare service staff

Validity of measures used: not reported

Notes

This material was based upon works supported by safefood, the Food Safety Promotion Board (under safefood grant no. 01-2008); in association with the Health Service Executive, Ireland.

\section{Risk of bias}

\begin{tabular}{lll}
\hline Bias & Authors' judgement & Support for judgement \\
\hline $\begin{array}{l}\text { Random sequence genera- } \\
\text { tion (selection bias) }\end{array}$ & Low risk & A random-number table was used to allocate services to treatment groups. \\
\hline $\begin{array}{l}\text { Allocation concealment } \\
\text { (selection bias) }\end{array}$ & Low risk & $\begin{array}{l}\text { We assumed that allocation was conducted in a single, automated process via } \\
\text { the random-number table and therefore allocation could not be pre-empted. }\end{array}$ \\
\hline $\begin{array}{l}\text { Blinding of participants } \\
\text { and personnel (perfor- } \\
\text { mance bias) }\end{array}$ & Unclear risk & $\begin{array}{l}\text { Due to nature of the intervention (training), childcare service staff and study } \\
\text { personnel delivering the intervention were not blind to study allocation, how- } \\
\text { ever, as both groups received some form of intervention, it was unknown if } \\
\text { there was a systematic difference in the potential for performance enhance- } \\
\text { ment and therefore bias. }\end{array}$ \\
\hline $\begin{array}{l}\text { Blinding of outcome as- } \\
\text { sessment (detection bias) }\end{array}$ & Unclear risk & $\begin{array}{l}\text { No information provided on whether the individuals conducting the outcome } \\
\text { assessment } \\
\text { (audits) were blind to group allocation }\end{array}$ \\
\hline $\begin{array}{l}\text { Incomplete outcome data } \\
\text { (attrition bias) }\end{array}$ & High risk & $\begin{array}{l}\text { Of } 31 \text { services allocated to the 'manager and staff training' intervention, only } \\
\text { All outcomes }\end{array}$ \\
$\begin{array}{l}\text { vices allocated to the 'manager training' group, 27 received the intervention } \\
\text { and } 24 \text { had follow-up data collected. Although data were provided to demon- } \\
\text { strate no significant difference between those who participated and did not, } \\
\text { this analysis was conducted for all services, not by group. Rated as high risk of } \\
\text { bias due to the magnitude of differences in participants lost to follow-up be- } \\
\text { tween groups }\end{array}$ \\
\hline
\end{tabular}

\begin{tabular}{|c|c|c|}
\hline $\begin{array}{l}\text { Selective reporting (re- } \\
\text { porting bias) }\end{array}$ & Unclear risk & $\begin{array}{l}\text { No prospective trial protocol or trial registration so it was unclear whether } \\
\text { there was selective outcome reporting. }\end{array}$ \\
\hline
\end{tabular}


Unit of allocation: childcare service

Unit of analysis: childcare service

Participants
Service type: Centre-based childcare services included preschools and

long daycare services

Region: Hunter region of New South Wales, Australia

\section{Demographic/socioeconomic characteristics:}

Children of aboriginal and/or Torres Strait Islander background enrolled

- Intervention: $68(56,80)$, control: $78(67,89)$

Service socioeconomic area

- Top 50\% of NSW: intervention $30(18,42)$, control $27(16,39)$

- Bottom $50 \%$ of NSW: intervention $70(58,82)$, control $73(61,84)$

Service geographical location

- Urban: intervention $50(37,63)$, control $59(46,72)$

- Rural: intervention 50 (37, 63), control $41(28,53$

Inclusion/exclusion criteria: Services in the region were ineligible if they: catered exclusively for children requiring specialist care, provided all on-site meals to children or were fully government funded, as the ethical clearance and intervention design were not appropriate for such services.

Number of services randomised: 128 services

\section{Numbers by trial group:}

$\mathrm{n}$ (controls baseline $)=64$

$\mathrm{n}$ (controls follow-up) $=60$

$\mathrm{n}$ (interventions baseline $)=64$

$\mathrm{n}$ (interventions follow-up) $=62$

\section{Recruitment:}

Service: 253 services were assessed for eligibility. Of these services, 56 did not meet the inclusion criteria and a further 67 declined to participate. Following the completion of baseline data collection, childcare services were randomly allocated to either the intervention or control condition by a research assistant using a random number function in a 1:1 (intervention: control) ratio.

Recruitment rate: $65 \%$

Interventions
Number of experimental conditions: 2 (intervention, control)

\section{Policies, practices or programmes targeted by the intervention:}

The healthy eating and physical activity policies and practices implemented by services included the following:

- Development of written nutrition and physical activity policies

- Staff monitoring of children's lunchboxes every day against written nutritional guidelines, provision of feedback to parents when a non-compliant food was packed

- Provision of water or reduced fat milk (for children over the age of 2 years) only 

- Staff role modelling of physically active play and healthy eating every day
- Staff provision of prompts and positive comments to children to encourage physical activity and healthy eating every day
- Provision of adult-guided fundamental movement skill development activities every day for at least $75 \%$ of children
- Restriction of sedentary screen time to less than weekly

\section{Implementation strategies:}

Opinion leaders: Nominated supervisors were asked to lead the development and implementation of nutrition and physical activity policies, co-facilitate training workshops with implementation support staff and communicate expectations regarding the implementation of policies and practices to childcare service staff.

Educational meetings: A series of three 1-h training workshops which focused on policy and practice implementation were provided on-site to childcare service staff and included both didactic and interactive components.

Local consensus process: Implementation support staff facilitated a discussion with nominated supervisors and childcare service staff to reach group agreement regarding an implementation strategy for the targeted policies and practices.

Educational outreach or academic detailing: Academic detailing visit was conducted which involved support staff observing and providing immediate feedback to childcare service staff as they implemented the practices targeted by the intervention.

Educational materials: All services received an electronic and hard copy package of tools and resources.

Audit and feedback: Verbal and written feedback describing service progress toward implementation of the targeted policies and practices was delivered at six intervals throughout the 12-month intervention.

Employment of a communications strategy: Services received hard copy and electronic bimonthly newsletters which communicated key messages relating to the healthy eating and physical activity policies and practices.

Implementation support staff: A support staff member provided ongoing implementation support and positive reinforcement via face-to-face visits, telephone and email contact.

Who delivered the intervention: Childcare staff members

Theoretical underpinning: The design of the intervention to support implementation of the policies and practices utilised Damschroder's Consolidated Framework for Implementation Research.

Description of control: The control group received three newsletters at the commencement, midpoint and conclusion of the 12-month intervention, containing information on healthy eating and physical activity unrelated to the specific policies and practices targeted by the intervention.

\section{Implementation of seven healthy eating and physical activity policies and practices}

Data collection method: questionnaire via a computer-assisted telephone interview

Validity of measures used: Nominated supervisors and room leaders were asked to report on their service's implementation of the seven healthy eating and physical activity policies and practices using items validated in a previous sample of 42 Australian childcare services. Agreement between nominated supervisor report and independent observation:

Presence of written nutrition $(75 \%, \mathrm{~K}=0.50)$ and physical activity policies $(79 \%, \mathrm{~K}=0.59)$. Staff monitoring of children's lunchboxes against written nutritional guidelines $(84 \%, \mathrm{~K}=0.69)$ and provision of

Strategies to improve the implementation of healthy eating, physical activity and obesity prevention policies, practices or programmes 
feedback to parents when a non-compliant food was packed $(68 \%, \mathrm{~K}=0.34)$. Provision of water $(89 \%$, $\mathrm{K}=0.78)$ or reduced fat milk only $(79 \%, \mathrm{~K}=0.57)$ to children. Staff role modelling of physically active play $(69 \%, \mathrm{~K}=0.39)$ and healthy eating $(94 \%, \mathrm{~K}=0.89)$ every day. Staff provision of prompts and positive comments to children to encourage physical activity $(80 \%, \mathrm{~K}=0.60)$ and healthy eating $(86 \%, \mathrm{~K}=$ $0.71)$ every day. Provision of adult-guided fundamental movement skill development activities ( $53 \%, \mathrm{~K}$ $=0.06)$ every day to at least $75 \%$ of children $(60 \%, K=0.20)$. Restriction of sedentary screen time $(58 \%$, $\mathrm{K}=0.17$ ) to less than weekly

Outcome relating to cost: not applicable

Outcome relating to adverse consequences:

Staff and child injury:

Data collection method: Nominated supervisors and room leader CATI

Validity of measures used: not reported

Outcome relating to child diet, physical activity or weight status:

Mean number of food groups consumed:

Data collection method: Child dietary intake was assessed during the 1-day observation using a modified version of the Dietary Observation for Child Care protocol.

Validity of measures used: The Dietary Observation for Child Care is a validated method for recording child level dietary intake in 2 to 5 year-olds and has been used extensively in the childcare setting.

Proportion of children engaged in sedentary, walking or very active physical activity during all observations, structured physical activity and outdoor free play sessions

Data collection method: Child physical activity levels were assessed at the same 1-day observation by the same observer, using a modified version of the System for Observing Play and Leisure in Youth (SOPLAY) tool and protocol.

Validity of measures used: SOPLAY has been found to be both valid and reliable in school-aged children and has been previously used to assess physical activity in the childcare setting.

Outcome relating to implementation strategy acceptability, adoption, penetration, sustainability and appropriateness:

Acceptability:

Data collection method: Nominated supervisors and room leader CATI

Validity of measures used: not reported

\section{Penetration:}

Data collection method: Project records maintained by each implementation support staff member

Validity of measures used: not reported

Notes

The research team acknowledges the funding support of the Australian National Preventive Health Agency (reference 95WOL2011), Hunter New England Population Health and Hunter Medical Research Institute.

\section{Risk of bias}

\begin{tabular}{lll}
\hline Bias & Authors' judgement & Support for judgement \\
\hline $\begin{array}{l}\text { Random sequence genera- } \\
\text { tion (selection bias) }\end{array}$ & Low risk & A random number function was used to generate the random sequence. \\
\hline
\end{tabular}

Strategies to improve the implementation of healthy eating, physical activity and obesity prevention policies, practices or programmes 
Jones 2015 (Continued)

$\begin{array}{ll}\begin{array}{l}\text { Allocation concealment } \\ \text { (selection bias) }\end{array} & \text { Low risk } \\ \end{array}$

Blinding of participants High risk
and personnel (performance bias)

All outcomes Services were not blind to study allocation and therefore high risk of performance bias.

\begin{tabular}{lll}
\hline $\begin{array}{l}\text { Blinding of outcome as- } \\
\text { sessment (detection bias) }\end{array}$ & High risk & $\begin{array}{l}\text { Services were not blind to study allocation and therefore high risk of detection } \\
\text { bias. }\end{array}$ \\
All outcomes &
\end{tabular}

\begin{tabular}{lll}
\hline $\begin{array}{l}\text { Incomplete outcome data } \\
\text { (attrition bias) } \\
\text { All outcomes }\end{array}$ & Low risk & 120/128 services (95\%) provided follow-up data. \\
\hline $\begin{array}{l}\text { Selective reporting (re- } \\
\text { porting bias) }\end{array}$ & Unclear risk & $\begin{array}{l}\text { The primary outcome was reported as prespecified, however the secondary } \\
\text { outcomes of child dietary intake and physical activity levels and adverse ef- } \\
\text { fects were not prespecified in the protocol paper. }\end{array}$ \\
\hline $\begin{array}{l}\text { Recruitment to cluster } \\
\text { Baseline imbalance }\end{array}$ & Low risk & $\begin{array}{l}\text { The children were randomly selected by asking the room leader at each ser- } \\
\text { vice to identify the three children with the most recent birthdays. }\end{array}$ \\
\hline $\begin{array}{l}\text { Loss of clusters } \\
\text { Incorrect analysis }\end{array}$ & $\begin{array}{l}\text { No baseline imbalances in service characteristics. No baseline measures of } \\
\text { secondary outcomes taken }\end{array}$ \\
\hline $\begin{array}{l}\text { Compatibility with individ- } \\
\text { ually randomised RCTs }\end{array}$ & Unclear risk & $\begin{array}{l}\text { Only follow-up data collected from random sample of services that had re- } \\
\text { mained in trial at follow-up }\end{array}$ \\
\hline
\end{tabular}

\section{Mazzucca 2017}

Study design: Cluster-RCT
Intervention duration: 10 weeks
Length of follow-up from baseline: Date of follow up data collection not specified
Differences in baseline characteristics: Reported
Unit of allocation: childcare service
Unit of analysis: Not reported
Service type: Childcare service
Region: Orange, Durham, Alamance and Guilford Counties, North Carolina, USA
Demographic/socioeconomic characteristics: Not reported
Inclusion/exclusion criteria: Eligible centres had at least a 2-star rating on NC's quality rating and im-
provement system. An additional eligibility criterion for this study was that centres had to have at least
one preschool classroom with children between 3-5 years of age and at least 10 preschool children en-
rolled in that classroom to ensure our ability to recruit sufficient numbers of children. Centres were

Strategies to improve the implementation of healthy eating, physical activity and obesity prevention policies, practices or programmes 
excluded if directors reported in the screening call that they were already providing the recommended 120 minutes of physical activity to children. Teachers were eligible for participation if they had not completed a programme to improve physical activity within the preceding six months and were willing to attend both in-person group workshops.

Number of services randomised: 26 services

\section{Numbers by trial group:}

$\mathrm{n}$ (controls baseline $)=13$

$\mathrm{n}$ (controls follow-up) $=13$

$\mathrm{n}$ (interventions baseline $)=13$

$\mathrm{n}$ (interventions follow-up) $=13$

\section{Recruitment:}

Service: 64 childcare centres were invited to participate, of which 17 centres did not meet the inclusion criteria and a further 21 centres refused to participate. Twenty-six ECE centre teachers (1 teacher per centre) were randomised 1:1 into either the intervention or waiting-list control arms. Randomisation took place after completion of all baseline measures.

Child: not reported

\section{Recruitment rate: $41 \%$}

Interventions

Number of experimental conditions: 2 (intervention, control)

\section{Policies, practices or programmes targeted by the intervention:}

The physical activity environment within centres including:

- Teachers encouraging children to be more active and less sedentary

- Teachers joining in active play with children

- Withholding of physical activity as punishment for bad behaviour

- Teachers reporting that they made portable play equipment available during play sessions

\section{Implementation strategies:}

Educational meetings: Workshops were held at the beginning and at the midpoint of the intervention period ( 5 weeks). Teachers attended two in-person, half-day workshops, which presented information about children's physical activity and sedentary behaviour at ECE centres based on prior research studies.

Educational materials: During both workshops, participants received intervention materials: MPL! activity lesson plans, activity cards corresponding to each MPL! activity, and $\$ 30$ worth of portable play equipment.

Classroom-based modules: After teachers completed the first in-person training workshop, they implemented intervention activities during four two-week modules. Within each module, newsletters, goal setting and self-monitoring, weekly technical assistance, and text message reminders were used to support implementation of classroom activities and teacher practices.

Newsletters: Sent at the beginning: each module reviewed information covered on that segment of the childcare day in the training workshop and gave them guidance on how to modify their behaviour to increase physical activity during that segment.

Educational outreach: Weekly technical assistance to each teacher through phone calls, emails, or text messages based on teacher preferences for communication to help teachers overcome challenges during implementation, increase their behavioural capacity and self-efficacy 
Tailored interventions: Teachers were reminded to set goals around the amount of time they would implement intervention activities and to share those with the interventionist.

Reminders: Teachers were sent text message reminders about implementing intervention activities about 2 times/week at the start of their day or during children's nap time.

Who delivered the intervention: Teachers

Theoretical underpinning: Not described

Description of control: Control group participants were asked to proceed according to their normal practices

\section{ECE centre physical activity and sedentary behaviour environment:}

Data collection method: Assessed using a modified version of the Environment and Policy Assessment and Observation - Self-Report (EPAO-SR) instrument. The EPAO-SR is a validated, comprehensive measure of both the nutrition and physical activity environments of childcare centres reported by centre directors and classroom teachers. Only items related to physical activity and sedentary behaviour were included in this study (149 items).

Validity of measures used: EPAO-SR is a validated measure.

Outcome relating to cost: not applicable

Outcome relating to adverse consequences: not applicable

Outcome relating to child diet, physical activity or weight status:

\section{Child physical activity:}

Data collection method: Accelerometer-measured minutes of children's non-sedentary time using GT3X accelerometers. Children wore accelerometers during waking hours for five childcare days at each data collection time point, which was used to estimate usual behaviour at each measurement point. Three days of wear for $\geq 4$ hours (excluding nap time) were required to be included in the analytic sample.

Validity of measures used: Objective measure of PA

\section{Child sedentary behaviour and intensities of physical activity:}

Data collection method:

As above and Epoch-level files were obtained using the ActiLife software, and data processing was done in SAS v9.4 using dates and times logged by teachers. Minutes per hour of sedentary behaviour and different intensities of physical activity were then calculated to account for differences in total wear time. An average of epoch-level counts per minute was calculated as an intensity-weighted daily average of physical activity.

Validity of measures used: Not described

Outcome relating to implementation strategy acceptability, adoption, penetration, sustainability and appropriateness

\section{Acceptability}

Data collection method: Data was collected via teacher self-report, interventionist report/logs, teacher surveys and exit interviews.

Validity of measures used: not described

\section{Penetration}


Mazzucca 2017 (Continued)

Data collection method: Data was collected via teacher self-report, interventionist report/logs, teacher surveys and exit interviews.

Validity of measures used: not described

Notes No sources of funding reported

\section{Risk of bias}

\begin{tabular}{|c|c|c|}
\hline Bias & Authors' judgement & Support for judgement \\
\hline $\begin{array}{l}\text { Random sequence genera- } \\
\text { tion (selection bias) }\end{array}$ & Unclear risk & The random sequence generation procedure was not described. \\
\hline $\begin{array}{l}\text { Allocation concealment } \\
\text { (selection bias) }\end{array}$ & Unclear risk & $\begin{array}{l}\text { There was no information provided about allocation concealment and there- } \\
\text { fore it was unclear if allocation was concealed. }\end{array}$ \\
\hline $\begin{array}{l}\text { Blinding of participants } \\
\text { and personnel (perfor- } \\
\text { mance bias) } \\
\text { All outcomes }\end{array}$ & High risk & $\begin{array}{l}\text { Measured using the Environment and Policy Assessment and Observation - } \\
\text { Self-report (EPAO-SR). There was no mention whether the teachers and child- } \\
\text { care personnel were blinded and therefore there was a high risk of perfor- } \\
\text { mance bias. }\end{array}$ \\
\hline \multirow[t]{2}{*}{$\begin{array}{l}\text { Blinding of outcome as- } \\
\text { sessment (detection bias) } \\
\text { All outcomes }\end{array}$} & High risk & $\begin{array}{l}\text { Outcome: Physical activity environment (measured using the Environment } \\
\text { and Policy Assessment and Observation - Self-report (EPAO-SR)) - teacher-re- } \\
\text { ported }\end{array}$ \\
\hline & & $\begin{array}{l}\text { There was no mention whether the teachers were blinded and therefore there } \\
\text { was a high risk of detection bias. }\end{array}$ \\
\hline $\begin{array}{l}\text { Incomplete outcome data } \\
\text { (attrition bias) } \\
\text { All outcomes }\end{array}$ & Low risk & $\begin{array}{l}\text { All } 13 \text { centres provided post-intervention data. Seven children ( } 4 \text { intervention, } \\
3 \text { control) of the } 182 \text { children did not provide post-intervention data ( } 4 \% \text { attri- } \\
\text { tion). }\end{array}$ \\
\hline $\begin{array}{l}\text { Selective reporting (re- } \\
\text { porting bias) }\end{array}$ & Low risk & The reported outcomes aligned with those outlined in the trial registration. \\
\hline Recruitment to cluster & Low risk & All children within participating centres/classes invited to participate \\
\hline Baseline imbalance & Low risk & No baseline imbalances in service characteristics or outcomes \\
\hline Loss of clusters & Low risk & No loss of sites \\
\hline Incorrect analysis & Low risk & Adjustment for potential clustering in analysis \\
\hline $\begin{array}{l}\text { Compatibility with individ- } \\
\text { ually randomised RCTs }\end{array}$ & Unclear risk & No evidence to make assessment \\
\hline
\end{tabular}

\section{Morshed 2016}

Methods

Study design: Controlled trial with a stratified, group-randomised design at the site level

Intervention duration: 2 years

Length of follow-up from baseline: 2 years (Fall 2008 to Spring 2010)

Differences in baseline characteristics: Reported

Unit of allocation: Childcare service

Strategies to improve the implementation of healthy eating, physical activity and obesity prevention policies, practices or programmes 
Unit of analysis: Childcare service

Participants

Service type: Childcare centres (Head Start centres)

Region: Rural New Mexico, USA

Demographic/socioeconomic characteristics: Head Start (HS) centres in American-Indian and predominantly Hispanic communities

Inclusion/exclusion criteria: Potential HS centres were identified for participation in the study based on the following criteria:

- Head Start centre enrolled predominantly Hispanic or American-Indian children

- Head Start centre enrolled a minimum of 20 3-year-old children

- Head Start retained at least $80 \%$ of its students for 2 years. HS centres in metropolitan areas were not eligible for inclusion, and HS centres within 150 miles of Albuquerque were prioritised to minimise travel expense.

$$
\begin{aligned}
& \text { Numbers by trial group: } \\
& n \text { (controls baseline })=8 \\
& n \text { (controls follow-up) }=8 \\
& n \text { (interventions baseline })=8 \\
& n \text { (interventions follow-up) }=8
\end{aligned}
$$

\section{Numbers by trial group:}

Number of services randomised: 16 services

\section{Recruitment:}

Service: 20 centres were recruited, of which 16 services participated. Centres were assigned to an intervention $(\mathrm{N}=8)$ or comparison $(\mathrm{N}=8)$ group after being stratified by ethnicity (American-Indian $\mathrm{N}=$ 6 , Hispanic $\mathrm{N}=10$ ) and HS BMI (lower-BMI $\leq 16.4, \mathrm{~N}=8$, higher BMI $>16.4, \mathrm{~N}=8$ ). The BMI cutoff point of 16.4 , chosen on the basis of a prerandomisation sample of 3-year-old children measured by centre staff, was close to the median and allowed a balanced distribution of HS centres within each racial/ethnic group. Among American-Indian centres, 3 were in each BMI group. Among Hispanic centres, 5 were in each BMI group. Within each of the 4 categories of median BMI and site ethnicity, centres were randomly assigned to intervention and comparison groups.

Recruitment rate: $80 \%$

Interventions

\section{Number of experimental conditions:}

2 (intervention, comparison)

Policies, practices or programmes targeted by the intervention:

- Increase structured physical activity by 30 minutes/day

- Provide repeated opportunities to try new fruit and vegetables

- Increase the variety of fruit and vegetables served

- Servings of target fruit and vegetables at least 4 times per quarter

- Increase the amount of whole-grain foods and low-fat dairy products served

- Improve food preparation methods

- Increase consumption of fruit, vegetables and whole grains

- Decrease consumption of sugar-sweetened beverages and high-fat foods

Strategies to improve the implementation of healthy eating, physical activity and obesity prevention policies, practices or programmes within childcare services (Review)

Copyright $\odot 2020$ The Cochrane Collaboration. Published by John Wiley \& Sons, Ltd. 
Morshed 2016 (Continued)

\author{
- Increase physical activity \\ - Decrease sedentary time \\ - Increase availability and visibility of healthier foods \\ - Provide nutrition information and recipes to HS families \\ - Reinforce CHILE messages during clinic visits and at HS family events \\ Implementation strategies:
}

Educational materials: The CHILE curriculum component formed the core of the intervention and consisted of nutrition and physical activity lessons delivered to children in classrooms.

Educational meetings: The foodservice staff received quarterly training aimed at making policy and behavioural changes to food purchasing and menus.

Who delivered the intervention: Staff

Theoretical underpinning: Socioecological Transcommunity model

Description of control: Comparison sites followed classroom activities and foodservice as usual.

- Total daily vegetable and whole-grain servings

- Grams of discretionary fat, which is fat in a food above the amount that would be found in a lean, lowfat, or fat-free form of the food; and teaspoons of added sugar provided, which are sugars added to foods during processing or preparation

- Grams of fat contributed daily by milk was calculated to measure changes in the fat content of milk

- Daily fruit servings (which excluded fruit juice from the fruit servings calculation)

Data collection method: Direct observations of foodservice staff during announced visits to HS sites. These data were collected in each HS centre during 5 weekdays. Observations of foodservice staff during all meals and snacks prepared on each data collection day using the CHILE Food Service Data Collection protocol and forms

Validity of measures used: Not reported

Outcome relating to cost: not applicable

Outcome relating to adverse consequences: not applicable

Outcome relating to child diet, physical activity or weight status: not applicable

Outcome relating to implementation strategy acceptability, adoption, penetration, sustainability and appropriateness: not applicable National Institutes of Health (\#1-R01DK72958-1).

\title{
Risk of bias
}

\begin{tabular}{lll}
\hline Bias & Authors' judgement & Support for judgement \\
\hline $\begin{array}{l}\text { Random sequence genera- } \\
\text { tion (selection bias) }\end{array}$ & Unclear risk & The random sequence generation procedure was not described. \\
\hline $\begin{array}{l}\text { Allocation concealment } \\
\text { (selection bias) }\end{array}$ & Unclear risk & $\begin{array}{l}\text { There was no information provided about allocation concealment and there- } \\
\text { fore it was unclear if allocation was concealed. }\end{array}$ \\
\hline \hline
\end{tabular}

Strategies to improve the implementation of healthy eating, physical activity and obesity prevention policies, practices or programmes 
Morshed 2016 (Continued)

Blinding of participants and personnel (perfor-

High risk mance bias)

All outcomes
Outcome: Observations of foodservice staff using the CHILE Food Service Data Collection protocol and forms. There was no mention that the participants and personnel were blinded and therefore there was a high risk of performance bias.

$\begin{array}{lll}\begin{array}{l}\text { Blinding of outcome as- } \\ \text { sessment (detection bias) }\end{array} & \text { Unclear risk } & \begin{array}{l}\text { Outcome: Observations of foodservice staff using the CHILE Food Service Data } \\ \text { Collection protocol and forms. There was no mention that the participants and } \\ \text { pll outcomes }\end{array} \\ & \begin{array}{l}\text { personnel were blinded however using audit of nutritional content of food and } \\ \text { therefore risk of detection bias was unclear. }\end{array}\end{array}$

Incomplete outcome data Low risk All recruited sites were retained throughout the study (from Cruz, p 8). (attrition bias)

All outcomes

Selective reporting (re- Unclear risk porting bias)

The associated papers did not explicitly state the primary and secondary outcomes and therefore it was unclear if there was selective outcome reporting (there was a secondary analysis not originally planned as part of the CHILE study $\mathrm{p}$ 418).

Intervention duration: 6 months

Length of follow-up from baseline: 9 months

Differences in baseline characteristics: Reported

Unit of allocation: By state

Unit of analysis: childcare service

Region: Columbia, South Carolina, and Raleigh, North Carolina, area.

Demographic/socioeconomic characteristics: South Carolina centres in the ABC Program served lowincome families. Centres in North Carolina that also served low-income families by accepting state subsidies were enrolled.

Inclusion/exclusion criteria: Centres were eligible to participate if they were classified by the state as a centre and not a family childcare home. Because South Carolina centres participating in the ABC Child Care Program served low-income families, centres in North Carolina also needed to serve lowincome families by accepting state subsidies to make the samples more comparable. Centres were excluded if they had an open case of abuse or neglect on file with either state.

\author{
Number of services randomised: 64 \\ Numbers by trial group: \\ $\mathrm{n}$ (controls baseline) $=30$ \\ $\mathrm{n}$ (controls follow-up) $=26$ \\ $\mathrm{n}$ (interventions baseline $)=34$ \\ $\mathrm{n}$ (interventions follow-up) $=33$
}

\title{
Recruitment:
}


O’Neill 2017 (Continued)

Service: Invitation letters were mailed to 342 centres, 174 eligible centres in South Carolina and 168 in North Carolina. The first 30 centres from each state that agreed to participate were enrolled. In the Columbia area, South Carolina, 34 centres were enrolled because of high interest. In the Raleigh area, North Carolina, 30 centres were enrolled.

Recruitment rate: $19 \%$
Number of experimental conditions: 2 (intervention, control)

\section{Policies, practices or programmes targeted by the intervention:}

Consistency with $\mathrm{ABC}$ Child Care programme nutrition standards:

- Only skim or $1 \%$ milk for children 2 y and older

- Sugar-sweetened beverages will not be served

- Juice allowed only once per day or less in 4-oz servings

- At least 2 different fruits served 2 or more times per day

- Vegetable other than white potatoes served at least 1 time per day

- Fried or pre-fried vegetables served 1 time per week or less

- Whole-grain foods served once per day

- High-fat meats served 2 times per week or less

- Sweet food items served 2 times per week or less

- Staff attend nutrition training at least 1 time per year

- Children learn about nutrition at least 1 time per week

- Do not use food as a reward or punishment

- Create and consistently implement a written nutrition policy

Physical activity practices related to 8 standards that applied to 3- to 5-year-old children, including:

- Encourage children to be physically active indoors and outdoors

- Create and consistently implement a written physical activity policy

- Require teachers to attend physical activity training at least once per year

- Do not use or withhold physical activity as punishment

- Implement 5 to 10 minutes of teacher-planned physical activity 2 or more times per day

- Provide active outdoor play, weather permitting, 2 to 3 times per day, totalling 90 to 120 minutes

- Provide a variety of play materials that promote physical activity indoors

- Provide a variety of play materials that promote physical activity outdoors

\section{Implementation strategies:}

Educational meetings: Four meetings were conducted across South Carolina to introduce directors to the standards, but no technical assistance or training was provided.

Release of guidelines/standards: South Carolina implemented 13 nutrition standards through the $A B C$ Child Care programme, a state-wide initiative to provide subsidised childcare to families in need. The nutrition standards applied to toddlers and preschoolers in care and targeted beverages, fruits and vegetables, whole grains, other foods, and policies and practices within the centres. 
O’Neill 2017 (Continued)

Who delivered the intervention: not reported

Theoretical underpinning: not reported

Description of control: usual practice

Outcomes

Outcome relating to the implementation of childcare service policies, practices or programmes:

Childcare centre implementation of physical activity practices:

Data collection method: Trained data collectors used the Environment and Policy Assessment and Observation (EPAO) tool to conduct observations in centres before implementation of the standards and 9 months after implementation. The EPAO assesses childcare physical activity environments, policies, and practices. It includes 8 physical activity subscales; the Physical Activity Environment Total Score is the mean of the subscale scores.

Validity of measures used: EPAO is a validated tool.

Childcare centre implementation of nutrition policies and practices:

Data collection method: Menu review (a component of the Environment and Policy Assessment and Observation) was used to evaluate the standards limiting sweet foods, high-fat meats, and fried or prefried vegetables, because these practices needed to be considered during the course of a full week. For the remaining food and beverage standards, data collected via the Diet Observation in Child Care were used to evaluate consistency with each standard.

Validity of measures used: The Environment and Policy Assessment and Observation assessed childcare nutrition environments, policies, and practices; the protocol and information about inter-rater reliability were reported elsewhere. The Diet Observation in Child Care (DOCC) was designed to assess foods and beverages served to three children in childcare settings and has demonstrated moderate to high reliability and validity.

Outcome relating to cost: not applicable

Outcome relating to adverse consequences: not applicable

Outcome relating to child diet, physical activity or weight status: not applicable

Outcome relating to implementation strategy acceptability, adoption, penetration, sustainability and appropriateness: not applicable

Notes

This study was supported, in part, by a grant from the Robert Wood Johnson Foundation (RWJF), Healthy Eating Research \#69551.

\section{Risk of bias}

\begin{tabular}{lll}
\hline Bias & Authors' judgement & Support for judgement \\
\hline $\begin{array}{l}\text { Random sequence genera- } \\
\text { tion (selection bias) }\end{array}$ & High risk & Study that involved no randomisation. Therefore high risk of selection bias \\
\hline $\begin{array}{l}\text { Allocation concealment } \\
\text { (selection bias) }\end{array}$ & High risk & $\begin{array}{l}\text { South Carolina centres were compared to North Carolina centres - no alloca- } \\
\text { tion concealment and high risk of selection bias }\end{array}$ \\
\hline $\begin{array}{l}\text { Blinding of participants } \\
\text { and personnel (perfor- } \\
\text { mance bias) } \\
\text { All outcomes }\end{array}$ & High risk & $\begin{array}{l}\text { Outcome: Environment and Policy Assessment and Observation (EPAO) scores } \\
\text { for physical activity (O'Neill 2017). There was no mention that participants and } \\
\text { personnel were blinded, therefore high risk of performance bias }\end{array}$ \\
\hline $\begin{array}{l}\text { Blinding of outcome as- } \\
\text { sessment (detection bias) } \\
\begin{array}{l}\text { All outcomes } \\
\hline \hline\end{array}\end{array}$ & Unclear risk & $\begin{array}{l}\text { Outcome: Environment and Policy Assessment and Observation (EPAO) scores } \\
\text { for physical activity (O'Neill 2017). It was unclear if personnel were blinded, } \\
\text { therefore unclear risk of detection bias }\end{array}$ \\
\hline \hline
\end{tabular}

Strategies to improve the implementation of healthy eating, physical activity and obesity prevention policies, practices or programmes 70 within childcare services (Review)

Copyright (c) 2020 The Cochrane Collaboration. Published by John Wiley \& Sons, Ltd. 
O'Neill 2017 (Continued)

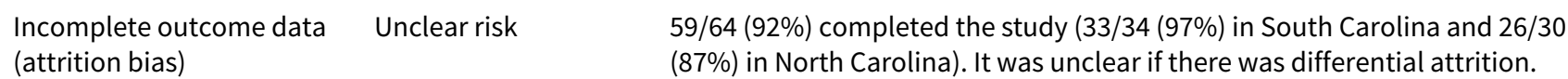

Alloutcomes

$(87 \%)$ in North Carolina). It was unclear if there was differential attrition.

\begin{tabular}{lll}
\hline $\begin{array}{l}\text { Selective reporting (re- } \\
\text { porting bias) }\end{array}$ & Unclear risk & $\begin{array}{l}\text { There was no study protocol therefore it was unclear if there was selective out- } \\
\text { come reporting. }\end{array}$ \\
\hline Baseline imbalance & Low risk & $\begin{array}{l}\text { Logistic regressions were conducted to evaluate consistency with each stan- } \\
\text { dard, adjusting for baseline and potential confounders. }\end{array}$ \\
\hline Potential confounding & Low risk & $\begin{array}{l}\text { Some baseline imbalances -adjustments made in analysis. Logistic regres- } \\
\text { sions were conducted to evaluate consistency with each standard, adjusting } \\
\text { for baseline and potential confounders. }\end{array}$ \\
\hline
\end{tabular}

Seward 2017

\begin{tabular}{ll}
\hline Methods & Study design: $\mathrm{RCT}$ \\
Intervention duration: 6 months \\
Length of follow-up from baseline: not reported \\
Differences in baseline characteristics: reported \\
Unit of allocation: Childcare service \\
Unit of analysis: Childcare service
\end{tabular}

Region: Hunter New England Local Health District. NSW, Australia

Demographic/socioeconomic characteristics: The Australian Statistical Geography Standard describes the region as encompassing non-metropolitan 'major cities' and 'inner regional' areas.

Major city + inner regional

- intervention $23.92 \%$

- control $17.85 \%$

Outer regional/remote Australia

- intervention $2.8 \%$

- control $2.10 \%$

Inclusion/exclusion criteria: Eligible childcare services were those that prepared and provided one main meal and two mid-meals to children while in care, and that were open for at least $8 \mathrm{~h} / \mathrm{d}$. Services that did not prepare and provide meals to children onsite or that did not have a cook with some responsibility for menu planning were excluded. Services catering exclusively for children requiring specialist care, mobile preschools and family daycare centres were also excluded, given the different operational characteristics of these services compared with centre-based long daycare services.

Number of services randomised: 54 childcare services

$$
\begin{aligned}
& \text { Numbers by trial group: } \\
& n \text { (controls baseline) }=20 \\
& n \text { (controls follow-up) }=20
\end{aligned}
$$


Seward 2017 (Continued)

$$
\begin{aligned}
& n(\text { interventions baseline })=25 \\
& n(\text { interventions follow-up })=24
\end{aligned}
$$

\section{Recruitment:}

Service: 106 services were assessed for eligibility. Of these, 16 services did not meet the inclusion criteria, 11 services declined to participate and a further 25 services were allocated to an alternative intervention. Consenting childcare services were immediately randomly allocated to an intervention or control group in a 1:1 ratio via block randomisation using a random number function in the statistical software package SAS version 9.3. Block size ranged between 2 and 6 .

\section{Recruitment rate: $88 \%$}

NB: twenty-five of the 79 services were allocated to receive an alternative intervention. They have been included in the recruitment rate.

\section{Implementation strategies:}

Opinion leaders: A memorandum of understanding outlining each party's responsibilities to implement the nutrition guidelines was signed by the implementation support officer, the service manager and the service cook.

Educational meetings: A one-day face-to-face menu-planning workshop was provided to service managers and cooks aiming to improve their knowledge and skills in the application of nutrition guidelines to childcare food service.

Educational materials: All intervention services received a resource pack to support the implementation of the nutrition guidelines which included the Caring for Children resource, menu-planning checklists, recipe ideas and budgeting fact sheets.

Audit and feedback: Intervention services had a dietitian complete an audit of their two-week menus at two time points, with written and verbal menu feedback provided at each time point.

Educational outreach or academic detailing: Support officer offered two face-to-face contacts with the service following the menu-planning workshop. In addition to the support visits, two newsletters were distributed to intervention services during the intervention period.

Who delivered the intervention: Long daycare service managers and service cooks

Theoretical underpinning: The Theoretical Domains Framework (TDF) was used to identify factors that influenced childcare services' implementation of nutrition guidelines.

Description of control: Services randomised to the control group were posted a hard copy of the Caring for Children resource and received usual care from the local health district health promotion staff.

\section{Compliance with nutrition guidelines:}

Data collection method: An independent dietitian, blinded to group allocation, assessed the menu and calculated servings of food groups per child based on the Australian Guide to Healthy Eating food groups. Menu compliance with nutrition guidelines was assessed via a menu assessment undertaken by a dietitian at baseline and follow-up.

Validity of measures used: not reported 
Outcome relating to cost: not applicable

Outcome relating to adverse consequences:

Negative feedback regarding service menu:

Data collection method: Pen and paper questionnaire

Validity of measures used: not reported

Average percentage of each meal not consumed by the children and classified as waste:

Data collection method: Pen and paper questionnaire

Validity of measures used: not reported

Outcome relating to child diet, physical activity or weight status:

Child food group consumption:

Data collection method: On the day of data collection, the research assistants collected the services' menu and the pre- and post-serving weights of two mid-meals (morning and afternoon tea) and one main meal (lunch).

Validity of measures used: Aggregated plate waste has been reported to be a valid method of assessing food intake at the group level and has been previously used in studies assessing the food intake of children in the school setting.

Outcome relating to implementation strategy acceptability, adoption, penetration, sustainability and appropriateness:

\section{Penetration:}

Data collection method: Project records maintained by implementation support staff were used to monitor the delivery of the intervention strategies.

Validity of measures used: not reported

Notes

This project was funded by the Priority Research Centre for Health Behaviour and received infrastructure funding from Hunter New England Population Health and the University of Newcastle. L.W. is supported by a National Health and Medical Research Council Career Development Fellowship and a Heart Foundation Future Leaders Fellowship.

\section{Risk of bias}

\begin{tabular}{lll}
\hline Bias & Authors' judgement & Support for judgement \\
\hline $\begin{array}{l}\text { Random sequence genera- } \\
\text { tion (selection bias) }\end{array}$ & Low risk & $\begin{array}{l}\text { A random number function in Microsoft Excel was used to generate the ran- } \\
\text { dom sequence. }\end{array}$ \\
\hline $\begin{array}{l}\text { Allocation concealment } \\
\text { (selection bias) }\end{array}$ & Low risk & $\begin{array}{l}\text { Consenting childcare services were immediately randomly allocated using a } \\
\text { random number function in the statistical software package SAS. }\end{array}$ \\
\hline $\begin{array}{l}\text { Blinding of participants } \\
\text { and personnel (perfor- } \\
\text { mance bias) } \\
\text { All outcomes }\end{array}$ & High risk & $\begin{array}{l}\text { Full compliance with nutritional guidelines, compliance with nutritional guide- } \\
\text { lines for individual AGHE food groups, menu compliance score, servings of } \\
\text { each food group provided }\end{array}$ \\
\hline
\end{tabular}

$\begin{array}{lll}\begin{array}{l}\text { Blinding of outcome as- } \\ \text { sessment (detection bias) }\end{array} & \text { Low risk } & \begin{array}{l}\text { Full compliance with nutritional guidelines, compliance with nutritional guide- } \\ \text { lines for individual AGHE food groups, menu compliance score, servings of } \\ \text { All outcomes }\end{array} \\ \text { each food group provided }\end{array}$


An independent dietitian blinded to group allocation assessed the menu and calculated servings of food groups per child based on the Australian Guide to Health Eating (AGHE) food groups.

\begin{tabular}{|c|c|c|}
\hline $\begin{array}{l}\text { Incomplete outcome data } \\
\text { (attrition bias) }\end{array}$ & Low risk & $\begin{array}{l}\text { At follow-up, } 24 / 26(92 \%) \text { in the intervention and } 20 / 28(71 \%) \text { in the control } \\
\text { group provided their menu. Missing data were imputed. }\end{array}$ \\
\hline
\end{tabular}

All outcomes

Selective reporting (re- Low risk The outcomes reported in the paper were prespecified in the protocol paper. porting bias)

Study design: nonrandomised trial
Intervention duration: 2 years
Length of follow-up from baseline: 2 years
Differences in baseline characteristics: reported
Unit of allocation: Childcare service
Unit of analysis: Childcare service

Region: Head Start centres in the intervention and comparison catchment areas in Houston and Austin, TX

Demographic/socioeconomic characteristics: Ethnically diverse population; lower median household income; and lower home ownership rates

\section{Inclusion/exclusion criteria: Not reported}

Number of services randomised: 25 centres

\section{Numbers by trial group:}

$\mathrm{n}$ (controls baseline $)=13$

$n$ (controls follow-up) $=13$

$\mathrm{n}$ (interventions baseline $)=12$

$\mathrm{n}$ (interventions follow-up) $=12$

Recruitment: Not reported

Recruitment rate: Not reported

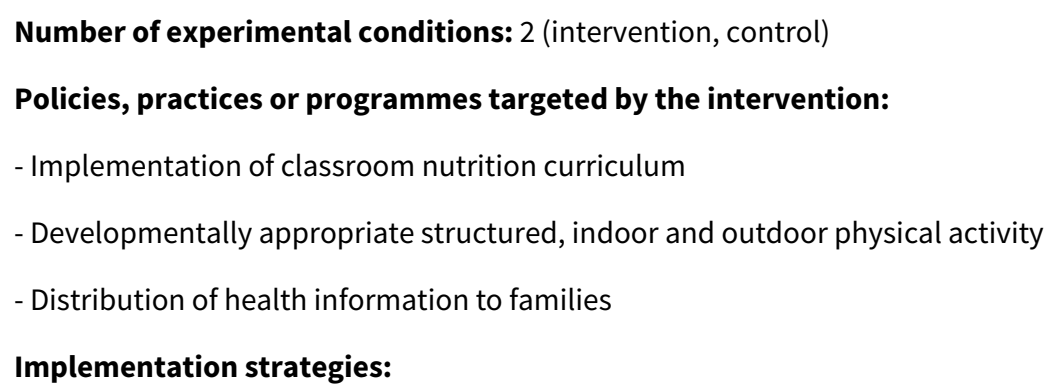


Educational meetings: Annual 6-hour training of centre teaching staff, centre directors, Head Start organisation level staff including wellness manager and nutrition manager. Booster training conducted twice a year in year 1 preschool staff who trained over a 4- to 6-hour training period. At the start of year 2 , another full training was conducted across the intervention centres for all teaching staff.

Reminders: Programme staff conducted technical support in the form of, monthly messages, and email reminders.

Academic detailing: In-person visits to the centres

Who delivered the intervention: Project staff

Theoretical underpinning: Social cognitive theory

Description of control: Usual practice

Data collection method: Teacher and centre director surveys reporting implementation of various CATCH EC programme components

Validity of measures used: not reported

Outcome relating to cost: not applicable

Outcome relating to adverse consequences: not applicable

Outcome relating to child diet, physical activity or weight status:

Child food intake frequency:

Data collection method: Parents completed surveys reporting child intake of various healthy and unhealthy foods, measuring child frequency of consumption of various foods including fruit, vegetables, French fries, sports drinks, water, and other sugar-sweetened beverages (e.g. sodas).

Validity of measures used: Not reported

\section{Child BMI:}

Data collection method: Child height and weight were measured using stadiometers and digital scales.

Validity of measured used: Not reported

Total child physical activity:

Data collection method: Parent-completed surveys reporting time spent in physical activity; parents were asked about their child's time spent in physical activity, including number of days per week they participated in more than 60 minutes of physical activity, and the number of days per week they played outside for 30 minutes.

Validity of measured used: Not reported

Outcome relating to implementation strategy acceptability, adoption, penetration, sustainability and appropriateness: not applicable port was provided by the Michael and Susan Dell Foundation through the Michael \& Susan Dell Center for Healthy Living. which has been funded, in part, with federal funds from the USDA/ARS under Cooperative Agreement number 58-6250-0-008.

\section{Risk of bias}

Strategies to improve the implementation of healthy eating, physical activity and obesity prevention policies, practices or programmes 
Sharma 2018 (Continued)

\begin{tabular}{lll} 
Bias & Authors' judgement & Support for judgement \\
\hline $\begin{array}{l}\text { Random sequence genera- } \\
\text { tion (selection bias) }\end{array}$ & High risk & $\begin{array}{l}\text { Nonrandomised study: nonrandom allocation (no random sequence generat- } \\
\text { ed). Therefore, high risk of selection bias }\end{array}$ \\
\hline $\begin{array}{l}\text { Allocation concealment } \\
\text { (selection bias) }\end{array}$ & High risk & $\begin{array}{l}\text { Nonrandomised study:- nonrandom allocation (no allocation concealment). } \\
\text { Therefore, high risk of selection bias }\end{array}$ \\
\hline $\begin{array}{l}\text { Blinding of participants } \\
\text { and personnel (perfor- } \\
\text { mance bias) }\end{array}$ & High risk & $\begin{array}{l}\text { CATCH EC implementation. There was no mention that the participants and } \\
\text { personnel were blinded and therefore there was a high risk of performance } \\
\text { bias. }\end{array}$ \\
\hline
\end{tabular}

Blinding of outcome as- High risk sessment (detection bias)

All outcomes
CATCH EC implementation. There was no mention of blinding and therefore there was a high risk of detection bias.

Incomplete outcome data Low risk No sites dropped out.
(attrition bias)

All outcomes

Selective reporting (re- Low risk All measures aligned between the Sharma and Hoelscher papers.
porting bias)

\begin{tabular}{lll}
\hline Recruitment to cluster & Low risk & All parents/children were invited to participate. \\
\hline Baseline imbalance & Unclear risk & $\begin{array}{l}\text { Significantly more parents in the intervention centres reported receiving SNAP } \\
\text { benefits compared with those in the comparison centres across both years } \\
\text { of measurement. For year 1, children in the comparison centres were slightly } \\
\text { younger than those in the intervention centres. }\end{array}$ \\
\end{tabular}

\begin{tabular}{lll}
\hline Loss of clusters & Low risk & No loss of clusters \\
\hline Incorrect analysis & Low risk & Adjustment for potential clustering in analysis \\
\hline $\begin{array}{l}\text { Compatibility with individ- } \\
\text { ually randomised RCTs }\end{array}$ & Unclear risk & No evidence to make assessment \\
\hline Potential confounding & Low risk & $\begin{array}{l}\text { Various known confounders that were considered for inclusion into each of } \\
\text { the regression models included: city (Houston and Austin), child race/ethnicity } \\
\text { and gender, parental race, and education level. }\end{array}$ \\
\hline
\end{tabular}

\section{Stookey 2017}

\begin{tabular}{ll}
\hline Methods & Study design: Cluster-RCT \\
& Intervention duration: 6 months \\
& Length of follow-up from baseline: 12 months \\
& Differences in baseline characteristics: reported \\
& Unit of allocation: Childcare service \\
& Unit of analysis: Childcare service \\
\hline Participants & Service type: Childcare service \\
\hline
\end{tabular}




\section{Region: San Francisco, USA}

Demographic/socioeconomic characteristics: The CCHP provides services to childcare centres that primarily serve low-income children in San Francisco and do not have federal, state or school district funding.

Inclusion/exclusion criteria: All childcare centres that participated in CCHP nutrition screenings in 2011-2012 were eligible for the HAP pilot. Childcare centres that were closed in Autumn 2012 or declined CCHP services for 2012-2013 before the randomisation were ineligible for the HAP pilot. Childcare centres with funding from Head Start, the San Francisco Unified School District, or Community College District were ineligible to receive CCHP screenings, and excluded from the HAP pilot.

Number of services randomised: 43 services

$$
\begin{aligned}
& \text { Numbers by trial group: } \\
& n \text { (controls baseline })=24 \\
& n \text { (controls follow-up) }=24 \\
& n \text { (interventions baseline) }=19 \\
& n \text { (interventions follow-up) }=19
\end{aligned}
$$

\section{Recruitment:}

Service: 45 childcare centres were invited to participate; of these, 43 centres participated. In summer 2012, the SFDPH epidemiologist randomised childcare centres in two blocks, one block for each of two $\mathrm{CCHP}$ health workers responsible for BMI screenings. A list of the same length of random, unique, unsorted numbers was generated using randomizer.org. For each health worker, childcare centres had an equal chance of being assigned to CCHP + HAP or CCHP + HAP Delayed. Enrolment in the childcare centres ranged from 14 to 160 children. The mean (SE) enrolment in childcare centres did not vary significantly by treatment assignment (48 (9) vs 37 (4)), and remained stable over time.

Child: 902 participants completed data collection at baseline. Of these, 522 were allocated to the intervention arm and 380 participants to the delayed control arm.

Recruitment rate: $96 \%$

Policies, practices or programmes targeted by the intervention:

- Use of physical activity curriculum

- Staff involvement in active play

- Visibility of pitchers of drinking water

Implementation strategies:

Educational materials: Invitation packet, which included information about the HAP, a self-assessment for childcare providers, and information about the gift card incentive for completing the self-assessment

Incentives: Gift card incentive for completing the self-assessment

Educational meetings: The San Francisco Children's Council offered two workshops to address needs identified by the HAP participants. A nutrition workshop addressed ideas for seasonal menu planning, child nutrition education resources for parents, and policies for food for holidays or celebrations. A physical activity workshop addressed how to integrate age-appropriate physical activity and academic learning for preschoolers.

Educational outreach or academic detailing: CCHP public health nurses or health workers introduced the HAP resources and process, in-person, to childcare centre staff. They delivered the HAP in-

Strategies to improve the implementation of healthy eating, physical activity and obesity prevention policies, practices or programmes 
vitation packet to the childcare centre, and spent up to $16 \mathrm{~h}$ per childcare centre, providing one-onone support to each childcare provider regarding the HAP self-assessment, goal setting, action plans to achieve the goal(s), Tip Sheets and online Technical Assistance resources.

Tailored interventions: The HAP translated the nutrition and physical activity NAPSACC resources and process into a programme that coordinated self-assessment and practice improvement across childcare providers.

Who delivered the intervention: Childcare centre staff

Theoretical underpinning: not reported

Description of control: Childcare centres allocated to the CCHP + HAP Delayed group were also offered HAP resources, only after a delay, in 2014-2015. Throughout the evaluation period, routine CCHP services were given to centres allocated to the CCHP + HAP Delayed group. These services included public health nurse consultation, health education, and hearing, vision, dental, and nutrition screenings and referrals.

Outcomes

Outcome relating to the implementation of childcare service policies, practices or programmes:

\section{Exposure to the 3 nutrition and physical activity centre index practices:}

Data collection method: The health workers gathered information about 3 practices which were relevant for tracking changes in response to HAP workshops that were offered in 2013. Data regarding the 3 index practices were combined into a score to track and compare cumulative changes in these practices in all CCHP + HAP and CCHP + HAP Delayed centres.

Validity of measures used: not reported

Outcome relating to cost: not applicable

Outcome relating to adverse consequences: not applicable

Outcome relating to child diet, physical activity or weight status:

Change in child BMI percentile at the child level and childcare centre level:

Data collection method: CCHP health workers visited all childcare centres that requested bi-annual BMI screenings in the autumn and spring of each academic year. The health workers recorded child age and sex, and measured child weight and height using a standardised protocol and calibrated instruments. Measurements were taken after the child removed outer layers of clothing and shoes. The age and sex-specific BMI percentile and BMI z-score for each child was calculated relative to the CDC 2000 growth reference using Epi Info 7 software. The change in BMI percentile was calculated. Incident cases of overweight or obesity were identified.

Validity of measures used: not reported

Outcome relating to implementation strategy acceptability, adoption, penetration, sustainability and appropriateness:

\section{Penetration:}

Data collection method: Collected by the San Francisco Children's Council Healthy Apple Program Coordinator, including number of childcare centres that completed the HAP self-assessment(s), set goals, received technical assistance materials, attended workshops, improved best practices, and received a HAP award.

Validity of measures used: not reported

The HAP development in 2011-2012 was funded by a CDC Community Transformation Grant. Funding for the HAP pilot evaluation was provided by the Feeling Good Project, funded by USDA SNAP-Ed, an equal opportunity provider and employer (Laura Brainin-Rodriguez, Coordinator).

Strategies to improve the implementation of healthy eating, physical activity and obesity prevention policies, practices or programmes 
Stookey 2017 (Continued)

Risk of bias

\begin{tabular}{lll}
\hline Bias & Authors' judgement & Support for judgement \\
\hline $\begin{array}{l}\text { Random sequence genera- } \\
\text { tion (selection bias) }\end{array}$ & Low risk & $\begin{array}{l}\text { A list of random, unique, unsorted numbers was generated using randomiz- } \\
\text { er.org. }\end{array}$ \\
\hline $\begin{array}{l}\text { Allocation concealment } \\
\text { (selection bias) }\end{array}$ & Low risk & $\begin{array}{l}\text { Eligible childcare centres were listed in alphabetical order and a list of random } \\
\text { numbers generated. }\end{array}$
\end{tabular}

Blinding of participants High risk and personnel (performance bias)

All outcomes
Outcome: 3 index practices (use of physical activity curriculum; staff usually joining in physical active play with children; pitchers of drinking water visible in the classroom).

The healthcare workers and childcare providers were not blinded to treatment allocation, therefore, there was a high risk of performance bias.

\begin{tabular}{|c|c|c|}
\hline $\begin{array}{l}\text { Blinding of outcome as- } \\
\text { sessment (detection bias) } \\
\text { All outcomes }\end{array}$ & High risk & $\begin{array}{l}\text { Outcome: } 3 \text { index practices (use of physical activity curriculum; staff usually } \\
\text { joining in physical active play with children; pitchers of drinking water visible } \\
\text { in the classroom). }\end{array}$ \\
\hline & & $\begin{array}{l}\text { The healthcare workers and childcare providers were not blinded to treatment } \\
\text { allocation, therefore, there was a high risk of detection bias. }\end{array}$ \\
\hline
\end{tabular}

Incomplete outcome data Low risk At the 2-year follow-up, 9 (4 in intervention, 5 in comparison) of the 43 centres (attrition bias)

All outcomes had missing data ( $21 \%$ attrition). Low risk of attrition bias

\begin{tabular}{|c|c|c|}
\hline $\begin{array}{l}\text { Selective reporting (re- } \\
\text { porting bias) }\end{array}$ & Unclear risk & $\begin{array}{l}\text { There was no study protocol, therefore, it was unclear if there was selective } \\
\text { outcome reporting. }\end{array}$ \\
\hline
\end{tabular}

\begin{tabular}{|c|c|c|}
\hline Recruitment to cluster & Low risk & All parents/children were invited to participate. \\
\hline Baseline imbalance & Unclear risk & $\begin{array}{l}\text { Some baseline imbalances, but unknown whether these biased outcome. } \\
\text { CCHP + HAP centres served significantly older children than CCHP + HAP De- } \\
\text { layed centres in 2011-2012 and 2012-2013. The CCHP + HAP centres had a sig- } \\
\text { nificantly smaller prevalence of overweight or obesity at autumn enrolment, } \\
\text { compared to CCHP + HAP Delayed centres, in the baseline year (2011-2012). } \\
\text { Intervention centres also had on average more children enrolled per centre } \\
\text { than control centres (i.e. difference in size). }\end{array}$ \\
\hline
\end{tabular}

\begin{tabular}{|c|c|c|}
\hline Loss of clusters & Low risk & Low risk of loss of clusters - similar \% of centres lost across groups \\
\hline Incorrect analysis & Low risk & $\begin{array}{l}\text { The intracluster correlation coefficient (ICC), measure of within-childcare cen- } \\
\text { ter variance relative to between-childcare center variance, was estimated to } \\
\text { describe clustering in the outcome data in the follow-up year and implementa- } \\
\text { tion year } 2 \text {. }\end{array}$ \\
\hline
\end{tabular}

\begin{tabular}{ll}
\hline Methods & Study design: RCT \\
& Intervention duration: 6 months \\
& Length of follow-up from baseline: 6 months \\
& Differences in baseline characteristics: reported
\end{tabular}

Strategies to improve the implementation of healthy eating, physical activity and obesity prevention policies, practices or programmes 
Ward 2008 (Continued)

Unit of allocation: childcare service

Unit of analysis: childcare service

Service type: childcare centres
Region: North Carolina, USA
Demographic/socioeconomic characteristics: not described
Inclusion/exclusion criteria: inclusion criteria: current enrolment of 15 to 150 children. Exclusion cri-
teria: services with an open case of abuse or neglect or served only a special population
Number of services randomised: 84 (56 intervention, 26 control, 2 excluded following randomisation)
Numbers by trial group:

$\mathrm{n}$ (controls baseline $)=26$

$\mathrm{n}$ (controls follow-up) $=26$

$\mathrm{n}$ (interventions baseline) $=56$

$\mathrm{n}$ (interventions follow-up) $=56$

Recruitment: all childcare health consultants working in North Carolina were invited to participate. A convenience sample was selected by recruiting the first 30 childcare health consultants (only 1 per county) who indicated an interest in participation, worked with at least 3 childcare services meeting eligibility requirements, and had not participated in the previous pilot project.

Recruitment rate: not reported

Interventions

Number of experimental conditions: 2 (intervention, control)

Policies, practices or programmes targeted by the intervention:

NAPSACC programme. Best practices for the promotion of proper nutrition and regular physical ac-

tivity at childcare. The programme focused on 15 nutrition and physical activity areas. Nutrition areas of focus included: fruits and vegetables; fried food and high-fat meats; beverages; menus and variety; meals and snacks; food items outside of regular meals and snacks; supporting healthful eating; nutrition education for children, parents and staff; and nutrition policy. Key physical activity areas of focus included: active play and inactive time; TV use and TV viewing; play environment; supporting physical activity; physical activity education for children, parents and staff; and physical activity policy.

Implementation strategies:

- Provision of educational materials

- Self-assessment instrument completed by service managers

- Action planning to improve at least 3 target areas identified from the self-assessment

- Education workshops on child being overweight, healthy eating and physical activity for children de-

livered by childcare health consultants

- Provision of technical assistance to service staff via in-person visits and telephone contact

- Re-assessment using the self-assessment tool

Who delivered the intervention: trained childcare health consultants

Theoretical underpinning: social cognitive theory against a social-ecologic framework

Description of control: delayed intervention control group

Outcomes

Outcome relating to the implementation of childcare service policies, practices or programmes:

- Total nutrition score

- Total physical activity score

Data collection method: EPAO tool including 1-day observation and a review of pertinent service documents conducted by trained observers. 75 items were selected to evaluate the impact of the intervention. All 75-item responses were converted to a 3-point scale (0, 1 and 2$)$, averaged within a given subscale, and multiplied by 10 , with the average of all subscale scores representing total nutrition and physical activity scores.

Validity of measures used: not established at time of study - additional work tests the reliability and validity of the NAPSACC self-assessment instrument in a sample of childcare services

Outcome relating to cost: not applicable

Outcome relating to adverse consequences: not applicable

Outcome relating to child diet, physical activity or weight status: not applicable

Outcome relating to implementation strategy acceptability, adoption, penetration, sustainability and appropriateness:

\section{Penetration:}


Ward 2008 (Continued)

Data collection method: not reported

Validity of measures used: not reported

Notes

This work was supported by a Potential Extramural Projects (PEP) grant from the CDC and a contract from the North Carolina Department of Health and Human Services, Division of Public Health.

\section{Risk of bias}

\begin{tabular}{lll}
\hline Bias & Authors' judgement & Support for judgement \\
\hline $\begin{array}{l}\text { Random sequence genera- } \\
\text { tion (selection bias) }\end{array}$ & Unclear risk & $\begin{array}{l}\text { No information provided on the method for generating random sequence for } \\
\text { allocation of childcare health consultants to treatment groups. }\end{array}$ \\
\hline $\begin{array}{l}\text { Allocation concealment } \\
\text { (selection bias) }\end{array}$ & Unclear risk & $\begin{array}{l}\text { No information provided on concealment of allocation of childcare health con- } \\
\text { sultants to groups. }\end{array}$ \\
\hline $\begin{array}{l}\text { Blinding of participants } \\
\text { and personnel (perfor- } \\
\text { mance bias) }\end{array}$ & High risk & $\begin{array}{l}\text { We assumed that, due to the nature of the intervention, childcare service staff } \\
\text { all outcomes } \\
\text { location and, therefore, there was a potential high risk of performance bias. }\end{array}$ \\
\hline $\begin{array}{l}\text { Blinding of outcome as- } \\
\text { sessment (detection bias) } \\
\text { All outcomes }\end{array}$ & Low risk & $\begin{array}{l}\text { Outcome assessors were blind to group allocation of services and the tool } \\
\text { used was } \\
\text { observational. }\end{array}$ \\
\hline $\begin{array}{l}\text { Incomplete outcome data } \\
\text { (attrition bias) } \\
\text { All outcomes }\end{array}$ & Low risk & $\begin{array}{l}82 \text { of } 84 \text { services recruited were followed up - } 2 \text { services were lost to follow-up } \\
\text { due to closure. }\end{array}$ \\
\hline $\begin{array}{l}\text { Selective reporting (re- } \\
\text { porting bias) }\end{array}$ & Unclear risk & $\begin{array}{l}\text { Authors stated that the outcome measures were determined a priori but un- } \\
\text { known if these were listed in a study protocol or trial registry. }\end{array}$ \\
\hline
\end{tabular}

Ward 2017

\begin{tabular}{ll}
\hline Methods & Study design: Cluster-RCT \\
Intervention duration: 4 months \\
Length of follow-up from baseline: 7 months \\
Differences in baseline characteristics: reported \\
Unit of allocation: Childcare service \\
Unit of analysis: Childcare service \\
\hline
\end{tabular}

Participants

Service type: Childcare service

Region: Three local ECE technical assistance organisations serving six counties in North Carolina agreed to assist with recruitment and Go NAPSACC implementation.

Demographic/socioeconomic characteristics: Largely rural and low-income areas, where resources are often limited

Inclusion/exclusion criteria: Eligible centres had to have children currently enrolled who were between 3 and 5 years and a quality rating of at least 2 stars (out of 5) or be faith-based (exempt from rating). Rating considers factors like teacher education, in-service training, teacher to-child ratios, and the 

cluded.

Number of services randomised: 33 centres

$$
\begin{aligned}
& \text { Numbers by trial group: } \\
& n \text { (controls baseline) }=15 \\
& n \text { (controls follow-up) }=14 \\
& n \text { (interventions baseline) }=18 \\
& n \text { (interventions follow-up) }=17
\end{aligned}
$$

\section{Recruitment:}

Service: 48 centres were invited to participate; of these 33 participated. Centres were randomly assigned $(1: 1)$ to receive either immediate access (intervention arm) or delayed access (control arm) to Go NAPSACC. Prior to randomisation, centres were stratified by county to ensure that each local agency would have half of its centres getting immediate access to the programme and half getting delayed access. Stratification by county also helped control for any potential differences between these geographic areas and their technical assistance staff that might influence implementation. A list of enrolled centres was provided to the study statistician, who then randomised participating centres into either intervention or control using a permutated block approach (block size of two to ensure equity between arms).

Recruitment rate: $69 \%$

- Childcare centre menus, food and beverages provided

- Childcare centre feeding environment

- Childcare centre feeding practices

- Staff education and professional development

- Childcare centre nutrition policy

\section{Implementation strategies:}

Audit and feedback: The self-assessment tool is an audit with feedback, allowing ECE programme administrators to evaluate their current performance. The action planning tool guides ECE programs to develop a formal implementation blueprint that will allow them to accomplish the goals they have set.

Educational materials: The tips and materials tool enables the distribution of educational materials that help ECE programme administrators as they implement their action plan and address any identified needs to provide education to teachers, parents, and children.

Tailored intervention: Presentation of results and goals are based on data supplied in the self-assessment to help the ECE programme administrator see where he/she is doing well and where there is room for improvement. Potential goals are also presented to the ECE programme administrator so he/ she can choose to work toward goals requiring small or large changes.

Educational outreach or academic detailing: One in-person meeting with the centre director to orient them to the Go NAPSACC tools (e.g. how to register for an account, complete a self-assessment, review results, set goals, create and customise action plans, navigate tips and materials). Following this orientation, TA providers conducted brief monthly check-ins by telephone or email (e.g. inquire about progress, assess need for additional assistance, remind about project timeline).

Who delivered the intervention: Childcare centre directors 
Ward 2017 (Continued)

Theoretical underpinning: Social cognitive theory

Description of control: delayed access to Go-NAPSACC

Outcomes

Outcome relating to the implementation of childcare service policies, practices or programmes:

Change in centres' nutrition environment:

Data collection method: Self-report version of the Environment and Policy Assessment and Observation (EPAO-SR) (SR = Self report) The EPAO-SR components include a Centre Director Questionnaire, Teacher Questionnaires, and a Policy Document Review

Validity of measures used: Reliability testing demonstrated day-to-day variation in things like foods and beverages served and teacher feeding practices (with ICCS of 0.06 to 0.60 ); however, reliability improved with multiple days of data capture (increasing ICCS to 0.20 to 0.86 ). Validity testing demonstrated generally good agreement between self-report and observation for foods and beverages served and nutrition policy (with correlations of 0.25 to 0.85 ), but lower agreement with teacher practices (correlations of 0.004 to 0.46$)$.

Outcome relating to cost: not applicable

Outcome relating to adverse consequences: not applicable

Outcome relating to child diet, physical activity or weight status: not applicable

Outcome relating to implementation strategy acceptability, adoption, penetration, sustainability and appropriateness:

\section{Acceptability:}

Data collection method: A sample of centre directors from the intervention arm $(n=6)$, completed a semi-structured interview.

Validity of measures used: not reported

\section{Penetration:}

Data collection method: Each local provider was asked to keep a log of their Go NAPSACC implementation activities.

Validity of measures used: not reported

Notes

Funding for this project was provided by a Healthy Eating Research grant from the Robert Wood Johnson Foundation and the Blue Cross Blue Shield of North Carolina Foundation. Additional support for this project came from the National Institutes of Health, National Institute of Nursing Research (T32NR007091). This project was conducted out of the Center for Health Promotion and Disease Prevention at the University of North Carolina at Chapel Hill, which is a Prevention Research Center funded through a Cooperative Agreement with the Centers for Disease Control and Prevention (U48DP005017).

\section{Risk of bias}

\begin{tabular}{lll}
\hline Bias & Authors' judgement & Support for judgement \\
\hline $\begin{array}{l}\text { Random sequence genera- } \\
\text { tion (selection bias) }\end{array}$ & Low risk & $\begin{array}{l}\text { A list of enrolled centres was provided to the study statistician who then ran- } \\
\text { domised participating centres into either intervention or control. }\end{array}$ \\
\hline $\begin{array}{l}\text { Allocation concealment } \\
\text { (selection bias) }\end{array}$ & Low risk & $\begin{array}{l}\text { Results of randomisation were shared with the study coordinator who then in- } \\
\text { formed participating centres. }\end{array}$ \\
\hline $\begin{array}{l}\text { Blinding of participants } \\
\text { and personnel (perfor- } \\
\text { mance bias) }\end{array}$ & High risk & $\begin{array}{l}\text { Outcome: Self-report version of the Environment and Policy Assessment and } \\
\text { Observation (EPAO-SR) \& EPAO-SR policy document review (completed by re- } \\
\text { search staff). }\end{array}$ \\
\hline
\end{tabular}

Strategies to improve the implementation of healthy eating, physical activity and obesity prevention policies, practices or programmes 
Ward 2017 (Continued)

All outcomes
There was no mention that the participants and personnel were blinded and, therefore, there was a high risk of performance bias.

\begin{tabular}{lll}
\hline Blinding of outcome as- & High risk & $\begin{array}{l}\text { Outcome: Self-report version of the Environment and Policy Assessment and } \\
\text { Observation (EPAO-SR) \& EPAO-SR policy document review (completed by re- } \\
\text { sessment (detection bias) }\end{array}$ \\
All outcomes & search staff).
\end{tabular}

All outcomes

There was no mention that the participants and personnel were blinded and, therefore, there was a high risk of detection bias.

\begin{tabular}{lll}
\hline $\begin{array}{l}\text { Incomplete outcome data } \\
\text { (attrition bias) } \\
\text { All outcomes }\end{array}$ & Low risk & $\begin{array}{l}2 \text { (1 intervention, } 1 \text { control) of the } 33 \text { centres (6\% attrition) failed to provide } \\
\text { data at follow-up. }\end{array}$ \\
\hline $\begin{array}{l}\text { Selective reporting (re- } \\
\text { porting bias) }\end{array}$ & Low risk & $\begin{array}{l}\text { The outcomes reported in the paper aligned with those listed in the trial regis- } \\
\text { tration. }\end{array}$ \\
\hline
\end{tabular}

Williams 2002

Study design: nonrandomised trial
Intervention duration: 3 years
Length of follow-up from baseline: 6 months, 18 months
Differences in baseline characteristics: not reported
Unit of allocation: childcare service
Unit of analysis: childcare service (child diet and weight status was assessed at the level of the indi-
vidual)

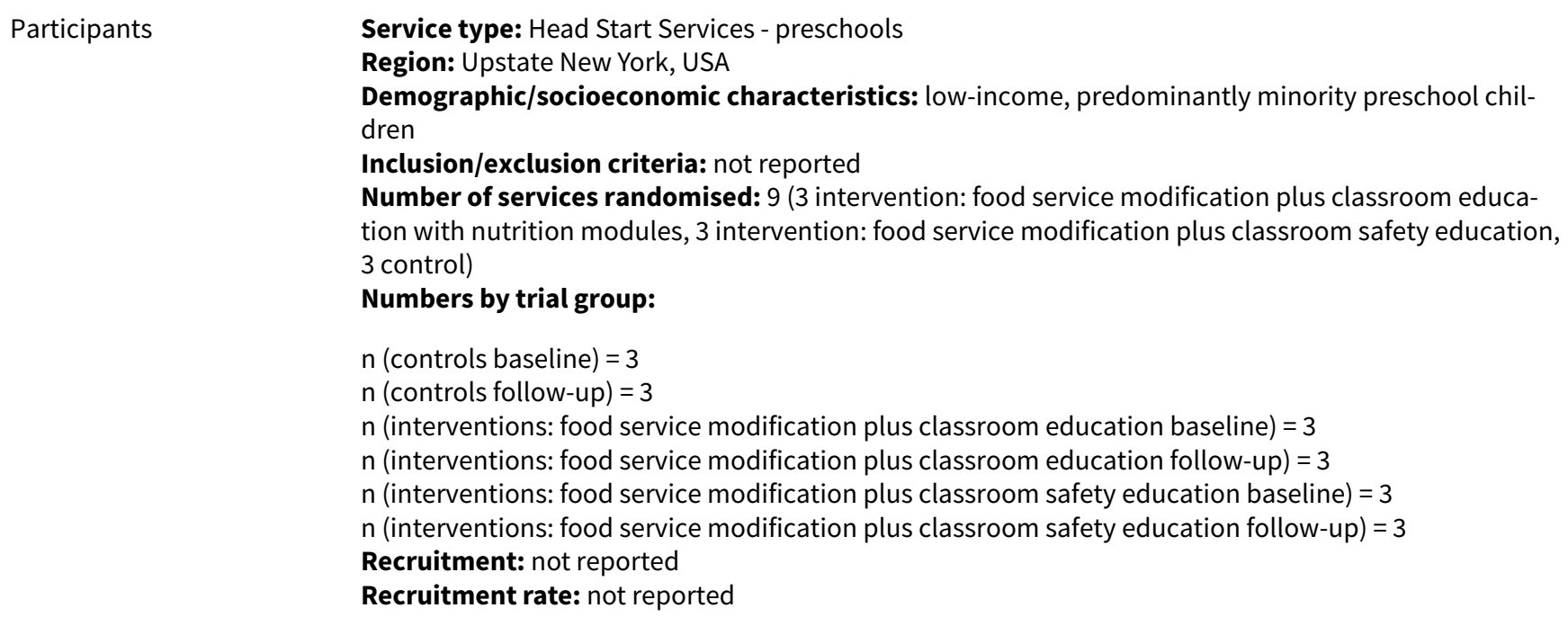

Participants

Service type: Head Start Services - preschools

Region: Upstate New York, USA

Demographic/socioeconomic characteristics: low-income, predominantly minority preschool children

Inclusion/exclusion criteria: not reported

Number of services randomised: 9 ( 3 intervention: food service modification plus classroom education with nutrition modules, 3 intervention: food service modification plus classroom safety education, 3 control)

\section{Numbers by trial group:}

$\mathrm{n}$ (controls baseline) $=3$

$\mathrm{n}$ (controls follow-up) $=3$

$\mathrm{n}$ (interventions: food service modification plus classroom education baseline) $=3$

$\mathrm{n}$ (interventions: food service modification plus classroom education follow-up) $=3$

$\mathrm{n}$ (interventions: food service modification plus classroom safety education baseline) $=3$

$\mathrm{n}$ (interventions: food service modification plus classroom safety education follow-up) $=3$

Recruitment: not reported

Recruitment rate: not reported

Number of experimental conditions: 3 (intervention: food service modification plus classroom education with nutrition modules, intervention: food service modification plus classroom safety education, control)

Policies, practices or programmes targeted by the intervention:

Food service modification:

- Achieving a 5-day a week meal/snack plan that provided no more than $30 \%$ energy from total fat and no more than $10 \%$ energy from saturated fat

- Increased offering of fruit, vegetables, breads and grains in meals, decreased total and saturated fat content of foods purchased for the service and decreased total and saturated fat due to alterations in food preparation techniques Implementation strategies: 
- Healthy Start Comprehensive Preschool Health Education Curriculum - core curriculum plus nutrition-related units

- 1-day training programme for cooks, which covered the major food service intervention areas: menu planning, recipe development, food purchasing and food preparation

- A list of objectives developed together with the cooks

- Ongoing support from registered dietitian

- Manual, newsletters and incentives

Intervention: food service modification plus classroom safety education:

- Healthy Start Comprehensive Preschool Health Education Curriculum - core curriculum plus safety-related unit

- 1-day training programme for cooks, which covered the major food service intervention areas: menu planning, recipe development, food purchasing and food preparation

- A list of objectives developed together with the cooks

- Ongoing support from registered dietitian

- Manual, newsletters and incentives

Who delivered the intervention: registered dietitians

Theoretical underpinning: not reported

Description of control: Healthy Start Comprehensive Preschool Health Education Curriculum - core curriculum plus safety-related units

Outcomes

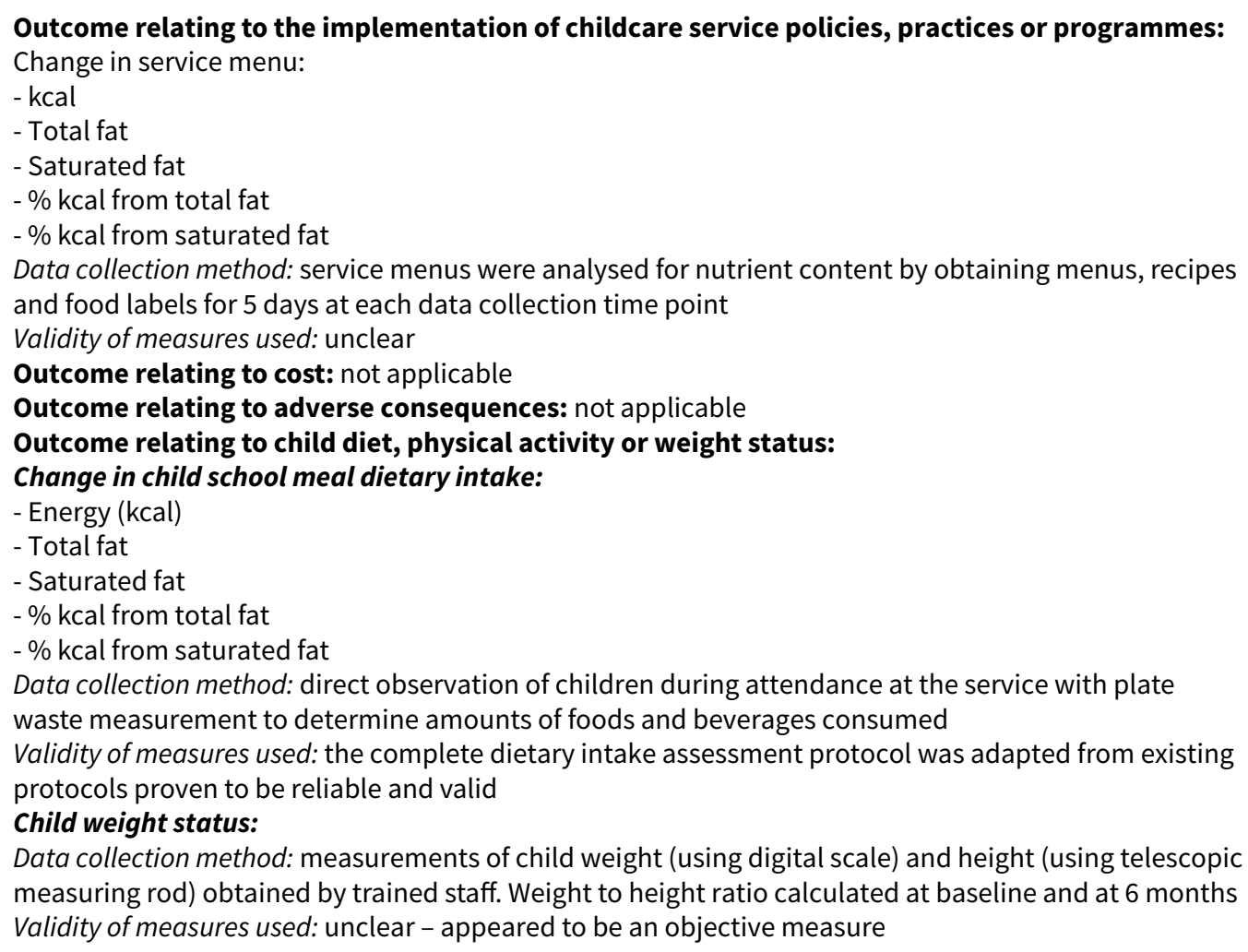

Outcome relating to implementation strategy acceptability, adoption, penetration, sustainability and appropriateness: not applicable

This research was funded by the National Heart Lung and Blood Institute, NIH, HL50321.

\section{Risk of bias}


Williams 2002 (Continued)

Random sequence genera- High risk No random allocation to control and intervention conditions (random allocation (selection bias) tion to 1 of 2 intervention conditions)

\begin{tabular}{l}
$\begin{array}{l}\text { Allocation concealment } \\
\text { (selection bias) }\end{array}$ High risk Unclear as to whether concealment of allocation occurred \\
\hline
\end{tabular}

\begin{tabular}{ll}
\hline $\begin{array}{l}\text { Blinding of participants } \\
\text { and personnel (perfor- }\end{array}$ & High risk \\
$\begin{array}{l}\text { mance bias) } \\
\text { All outcomes }\end{array}$ & $\begin{array}{l}\text { We assumed that, due to the nature of the intervention, childcare service staff } \\
\text { and study personnel delivering the intervention were not blind to the study al- } \\
\text { location and, therefore, there was a potential high risk of performance bias. }\end{array}$
\end{tabular}

\begin{tabular}{|c|c|c|}
\hline $\begin{array}{l}\text { Blinding of outcome as- } \\
\text { sessment (detection bias) } \\
\text { All outcomes }\end{array}$ & Unclear risk & $\begin{array}{l}\text { No information was provided on whether research personnel undertaking } \\
\text { menu assessment } \\
\text { and other data collection were blind to group allocation. }\end{array}$ \\
\hline $\begin{array}{l}\text { Incomplete outcome data } \\
\text { (attrition bias) } \\
\text { All outcomes }\end{array}$ & Low risk & $\begin{array}{l}\text { Implementation data collected on all intervention }(n=6) \text { and control services } \\
(n=3) \text { pre- and post-intervention }\end{array}$ \\
\hline $\begin{array}{l}\text { Selective reporting (re- } \\
\text { porting bias) }\end{array}$ & Low risk & $\begin{array}{l}\text { Methodology paper also listed physiological measures - these were published } \\
\text { elsewhere. }\end{array}$ \\
\hline Potential confounding & Unclear risk & No information provided \\
\hline
\end{tabular}

\title{
Yoong 2016
}

\section{Methods}

Study design: parallel-group RCT

Intervention duration: $6-8$ weeks

Length of follow-up from baseline: no baseline data collection

Differences in baseline characteristics: reported

Unit of allocation: Childcare service

Unit of analysis: Childcare service

\section{Participants}

Service type: Long daycare services

Region: NSW, Australia

Demographic/socioeconomic characteristics: Not stated

Inclusion/exclusion criteria: Long daycare services (centre-based services typically open $\geq 8$ hours/ day) located within NSW, Australia, served as the sampling frame. Services were excluded if they did not undertake menu planning on site or where cooks did not understand English sufficiently to complete the survey.

\author{
Numbers by trial group: \\ $\mathrm{n}$ (controls baseline $)=\mathrm{n} / \mathrm{a}$ \\ $\mathrm{n}$ (controls follow-up) $=39$ \\ $\mathrm{n}$ (interventions baseline $)=\mathrm{n} / \mathrm{a}$ \\ $\mathrm{n}$ (interventions follow-up) $=38$
}

Number of services randomised: 77 services

Strategies to improve the implementation of healthy eating, physical activity and obesity prevention policies, practices or programmes 


\section{Recruitment:}

Service: 220 services were invited to participate; of these 106 were ineligible to participate, 34 declined to participate and a further 14 could not be contacted. Seventy-seven consented to participate and were randomly allocated to either the intervention or control condition by a blinded research assistant using a random number function in Microsoft Excel in a 1:1 ratio.

Recruitment rate: $68 \%$

Interventions

Number of experimental conditions: 2 (intervention, control)

Policies, practices or programmes targeted by the intervention:

- Provision of fruit and vegetables on service menu

\section{Implementation strategies:}

Educational materials: Intervention cooks were mailed a two-page education resource and the menu planning checklist from the Caring for Children resource.

Who delivered the intervention: Printed resources were developed by a local health promotion team consisting of dietitians, behavioural scientists and health promotion practitioners.

Theoretical underpinning: The content of the material was guided by the theory of planned behaviour (TPB).

Description of control: The control group received usual care. All services could access the Caring for Children resource online and may have been offered support from their local health promotion staff.

\section{Provision of fruit and vegetables:}

Data collection method: A one-item question was used to assess the provision of fruit and vegetables on menus.

Validity of measures used: This measure was not validated and is likely to result in an overestimation of effect.

Outcome relating to cost: not applicable

Outcome relating to adverse consequences: not applicable

Outcome relating to child diet, physical activity or weight status: not applicable

Outcome relating to implementation strategy acceptability, adoption, penetration, sustainability and appropriateness:

\section{Penetration:}

Data collection method: A telephone interview where participants were asked whether they recalled receiving the educational material and, if so, how long ago they received it

Validity of measures used: not reported 
Yoong 2016 (Continued)

Random sequence genera- Low risk A random number function in Microsoft Excel was used to generate the rantion (selection bias) dom sequence.

\begin{tabular}{|c|c|c|}
\hline $\begin{array}{l}\text { Allocation concealment } \\
\text { (selection bias) }\end{array}$ & Low risk & $\begin{array}{l}\text { Microsoft Excel was used to generate a list of random numbers and could } \\
\text { forsee assignment. }\end{array}$ \\
\hline
\end{tabular}

\begin{tabular}{|c|c|c|}
\hline $\begin{array}{l}\text { Blinding of participants } \\
\text { and personnel (perfor- } \\
\text { mance bias) }\end{array}$ & High risk & $\begin{array}{l}\text { Primary outcome of review: Number of fruit and vegetables provided on menu } \\
\text { in last week. There was no blinding to group allocation of participants de- } \\
\text { scribed and this was likely to influence performance. }\end{array}$ \\
\hline
\end{tabular}

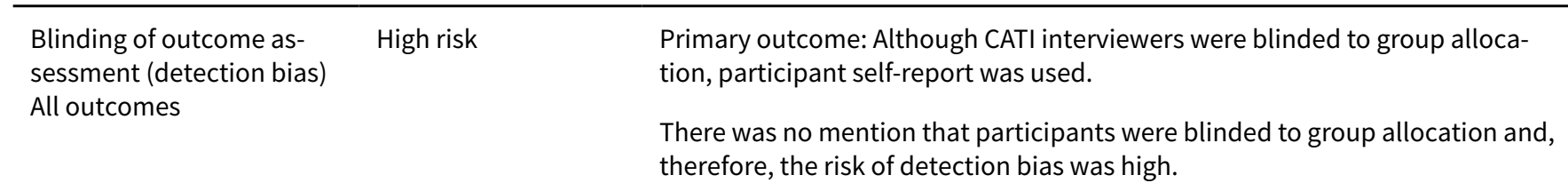

\begin{tabular}{|c|c|c|}
\hline $\begin{array}{l}\text { Incomplete outcome data } \\
\text { (attrition bias) } \\
\text { All outcomes }\end{array}$ & Low risk & $\begin{array}{l}\text { Table } 2 \text { outlines that there was missing data for two services }(2 / 77=3 \%) \text { and } \\
\text { therefore there was a low risk of attrition bias. }\end{array}$ \\
\hline $\begin{array}{l}\text { Selective reporting (re- } \\
\text { porting bias) }\end{array}$ & Low risk & $\begin{array}{l}\text { The measures reported in the trial registration aligned with those reported in } \\
\text { the outcome paper. }\end{array}$ \\
\hline
\end{tabular}

ABC: Activity Begins in Childhood

AGHE: Australian Guide to Healthy Eating

BMI: Body Mass Index

CATCH EC: Coordinated Approach to Child Health Early Childhood

CATI: Computer Assisted Telephone Interview

CCHP: Child Care Health Program

CDC: Centers for Disease Control

CHILE: Child Initiative for Lifelong Eating and Exercise

CHPHSPC: Californian Childcare Health Programme Health and Safety Checklist

CSPlan: Complex Samples Plan

DOCC: Diet Observation in Child Care

DVD: Digital Versatile Disc

ECE: Early Care and Education

EPAO: Environment and Policy Assessment and Observation

EPAO-SR: Environment and Policy Assessment and Observation Self Report

HAP: Healthy Apple Program

HS: Head Start

IOM: Institute of Medicine

ICC: Intraclass Correlation Coefficient

MPL!: Move, Play, Learn!

NAPSACC: Nutrition and Physical Activity Self-Assessment for Child Care

NC: North Carolina

OSRAP: Observation System for Recording Activity in Preschools

PA: Physical Activity

RCT: Randomised Controlled Trial

SE: Standard Error

SFDPH: San Francisco Department of Public Health

SNAP: Supplementation Nutrition Assistance Program

SOPLAY: System for Observing Play and Leisure in Youth

TA: Technical Assistant

TBP:Theory of Planned Behaviour

TDF: theoretical domains framework

Strategies to improve the implementation of healthy eating, physical activity and obesity prevention policies, practices or programmes 
vs: Versus

ZBMI: z Body Mass Index

Characteristics of excluded studies [ordered by study ID]

\begin{tabular}{|c|c|}
\hline Study & Reason for exclusion \\
\hline Adamo 2015 & $\begin{array}{l}\text { Inappropriate outcomes - did not aim to improve implementation of a policy, programme or prac- } \\
\text { tice }\end{array}$ \\
\hline Adamo 2017 & $\begin{array}{l}\text { Inappropriate outcomes - did not aim to improve implementation of a policy, programme or prac- } \\
\text { tice }\end{array}$ \\
\hline Bardid 2017 & $\begin{array}{l}\text { Inappropriate outcomes - did not aim to improve implementation of a policy, programme or prac- } \\
\text { tice }\end{array}$ \\
\hline Bell 2015 & Non-controlled study \\
\hline Birnbaum 2017 & $\begin{array}{l}\text { Inappropriate outcomes - did not aim to improve implementation of a policy, programme or prac- } \\
\text { tice }\end{array}$ \\
\hline Brand 2017 & $\begin{array}{l}\text { Inappropriate outcomes - did not aim to improve implementation of a policy, programme or prac- } \\
\text { tice }\end{array}$ \\
\hline Brian 2017a & $\begin{array}{l}\text { Inappropriate outcomes - did not aim to improve implementation of a policy, programme or prac- } \\
\text { tice }\end{array}$ \\
\hline Brian 2017b & $\begin{array}{l}\text { Inappropriate outcomes - did not aim to improve implementation of a policy, programme or prac- } \\
\text { tice }\end{array}$ \\
\hline Burkart 2018 & $\begin{array}{l}\text { Inappropriate outcomes - did not aim to improve implementation of a policy, programme or prac- } \\
\text { tice }\end{array}$ \\
\hline Byun 2018 & $\begin{array}{l}\text { Inappropriate outcomes - did not aim to improve implementation of a policy, programme or prac- } \\
\text { tice }\end{array}$ \\
\hline Chuang 2018 & $\begin{array}{l}\text { Inappropriate outcomes - did not aim to improve implementation of a policy, programme or prac- } \\
\text { tice }\end{array}$ \\
\hline Davis 2016 & $\begin{array}{l}\text { Inappropriate outcomes - did not aim to improve implementation of a policy, programme or prac- } \\
\text { tice }\end{array}$ \\
\hline De Craemer 2017 & $\begin{array}{l}\text { Inappropriate outcomes - did not aim to improve implementation of a policy, programme or prac- } \\
\text { tice }\end{array}$ \\
\hline Driediger 2018 & $\begin{array}{l}\text { Inappropriate outcomes - did not aim to improve implementation of a policy, programme or prac- } \\
\text { tice }\end{array}$ \\
\hline Foulkes 2017 & $\begin{array}{l}\text { Inappropriate outcomes - did not aim to improve implementation of a policy, programme or prac- } \\
\text { tice }\end{array}$ \\
\hline Gelli 2017 & $\begin{array}{l}\text { Inappropriate participants - did not include childcare services (e.g. study targets primary or sec- } \\
\text { ondary schools) }\end{array}$ \\
\hline Goldfield 2016 & $\begin{array}{l}\text { Inappropriate outcomes - did not aim to improve implementation of a policy, programme or prac- } \\
\text { tice }\end{array}$ \\
\hline
\end{tabular}




\begin{tabular}{|c|c|}
\hline Study & Reason for exclusion \\
\hline Hu 2017 & $\begin{array}{l}\text { Inappropriate outcomes - did not aim to improve implementation of a policy, programme or prac- } \\
\text { tice }\end{array}$ \\
\hline ISRCTN94022291 & $\begin{array}{l}\text { Inappropriate outcomes - did not aim to improve implementation of a policy, programme or prac- } \\
\text { tice }\end{array}$ \\
\hline Jones 2016 & $\begin{array}{l}\text { Inappropriate outcomes - did not aim to improve implementation of a policy, programme or prac- } \\
\text { tice }\end{array}$ \\
\hline Lau 2017 & $\begin{array}{l}\text { Inappropriate outcomes - did not aim to improve implementation of a policy, programme or prac- } \\
\text { tice }\end{array}$ \\
\hline Lumeng 2017 & $\begin{array}{l}\text { Inappropriate outcomes - did not aim to improve implementation of a policy, programme or prac- } \\
\text { tice }\end{array}$ \\
\hline Malden 2018 & $\begin{array}{l}\text { Inappropriate outcomes - did not aim to improve implementation of a policy, programme or prac- } \\
\text { tice }\end{array}$ \\
\hline Mattingly 2016 & Non-controlled study \\
\hline McSweeney 2017 & $\begin{array}{l}\text { Inappropriate outcomes - did not aim to improve implementation of a policy, programme or prac- } \\
\text { tice }\end{array}$ \\
\hline Natale 2017 & $\begin{array}{l}\text { Inappropriate outcomes - did not aim to improve implementation of a policy, programme or prac- } \\
\text { tice }\end{array}$ \\
\hline NCT02789215 & $\begin{array}{l}\text { Inappropriate outcomes - did not aim to improve implementation of a policy, programme or prac- } \\
\text { tice }\end{array}$ \\
\hline NCT03022472 & $\begin{array}{l}\text { Inappropriate outcomes - did not aim to improve implementation of a policy, programme or prac- } \\
\text { tice }\end{array}$ \\
\hline NCT03713840 & $\begin{array}{l}\text { Inappropriate outcomes - did not aim to improve implementation of a policy, programme or prac- } \\
\text { tice }\end{array}$ \\
\hline Nezami 2018 & $\begin{array}{l}\text { Inappropriate participants - did not include childcare services (e.g. study targets primary or sec- } \\
\text { ondary schools) }\end{array}$ \\
\hline Pate 2016 & $\begin{array}{l}\text { Inappropriate outcomes - did not aim to improve implementation of a policy, programme or prac- } \\
\text { tice }\end{array}$ \\
\hline Pinket 2016 & $\begin{array}{l}\text { Inappropriate outcomes - did not aim to improve implementation of a policy, programme or prac- } \\
\text { tice }\end{array}$ \\
\hline Razak 2018 & $\begin{array}{l}\text { Inappropriate intervention - did not aim to improve the implementation of policies, practices or } \\
\text { programmes by usual childcare service staff }\end{array}$ \\
\hline Roth 2015 & $\begin{array}{l}\text { Inappropriate outcomes - did not aim to improve implementation of a policy, programme or prac- } \\
\text { tice }\end{array}$ \\
\hline Truelove 2016 & $\begin{array}{l}\text { Inappropriate outcomes - did not aim to improve implementation of a policy, programme or prac- } \\
\text { tice }\end{array}$ \\
\hline Truelove 2018 & $\begin{array}{l}\text { Inappropriate outcomes - did not aim to improve implementation of a policy, programme or prac- } \\
\text { tice }\end{array}$ \\
\hline
\end{tabular}




\begin{tabular}{ll}
\hline Study & Reason for exclusion \\
\hline Tucker 2016 & $\begin{array}{l}\text { Inappropriate outcomes - did not aim to improve implementation of a policy, programme or prac- } \\
\text { tice }\end{array}$ \\
\hline Tucker 2017 & $\begin{array}{l}\text { Inappropriate outcomes - did not aim to improve implementation of a policy, programme or prac- } \\
\text { tice }\end{array}$ \\
\hline Vanderloo 2016 & $\begin{array}{l}\text { Inappropriate outcomes - did not aim to improve implementation of a policy, programme or prac- } \\
\text { tice }\end{array}$ \\
\hline
\end{tabular}

Characteristics of ongoing studies [ordered by study ID]

Helland 2016

Trial name or title Study protocol for a multi-component kindergarten-based intervention to promote healthy diets in toddlers: a cluster-randomised trial

\begin{tabular}{|c|c|}
\hline Methods & Study design: Cluster-randomised controlled trial \\
\hline \multirow[t]{3}{*}{ Participants } & Service type: Kindergarten departments for toddlers \\
\hline & Region: Vest-Agder and Aust-Agder counties of Norway \\
\hline & Number of services participating: 18 services \\
\hline \multirow[t]{6}{*}{ Interventions } & Number of experimental conditions: 2 (intervention, control) \\
\hline & Policies, practices or programmes targeted by the intervention: \\
\hline & - Kindergarten personnel feeding practices \\
\hline & - Ten meal principles regarding responsive feeding and food joy in the meal setting \\
\hline & Implementation strategies: \\
\hline & - Educational meetings \\
\hline \multirow[t]{2}{*}{ Outcomes } & $\begin{array}{l}\text { Outcome relating to the implementation of childcare service policies, practices or pro- } \\
\text { grammes: }\end{array}$ \\
\hline & - Implementation of kindergarten staff feeding practices \\
\hline Starting date & Sissel H. Helland. sissel.h.hellandg@gmail.com \\
\hline \multirow[t]{2}{*}{ Contact information } & Trial registration: ISRCTN74823448 \\
\hline & DOI 10.1186/ISRCTN74823448 \\
\hline Notes & Sissel H. Helland. sissel.h.hellandg@gmail.com \\
\hline
\end{tabular}

Hennink-Kaminskia 2017

$\begin{array}{ll}\text { Trial name or title } & \begin{array}{l}\text { Parent and childcare provider partnerships: Protocol for the Healthy Me, Healthy We (HMHW) clus- } \\ \text { ter-randomised control trial }\end{array}\end{array}$


Hennink-Kaminskia 2017 (Continued)

\begin{tabular}{|c|c|}
\hline Methods & Study design: Cluster-randomised controlled trial \\
\hline \multirow[t]{3}{*}{ Participants } & Service type: Childcare centres with dedicated classrooms for 3-4-year-olds \\
\hline & Region: Rural and suburban counties of North Carolina \\
\hline & Number of services participating: 96 services \\
\hline \multirow[t]{7}{*}{ Interventions } & Number of experimental conditions: 2 (intervention, waiting-list control) \\
\hline & Policies, practices or programmes targeted by the intervention: \\
\hline & - Nutrition and physical activity practices \\
\hline & Implementation strategies: \\
\hline & - Two training sessions delivered face-to-face for childcare services \\
\hline & - Check-in visits to childcare services \\
\hline & - Provision of resources to childcare services \\
\hline \multirow[t]{2}{*}{ Outcomes } & $\begin{array}{l}\text { Outcome relating to the implementation of childcare service policies, practices or pro- } \\
\text { grammes: }\end{array}$ \\
\hline & $\begin{array}{l}\text { Change in childcare centres' environmental characteristics related to nutrition and physical activi- } \\
\text { ty will be assessed using the Environment and Policy Assessment and Observation (EPAO) }\end{array}$ \\
\hline Starting date & June 2017 \\
\hline Contact information & Heidi Hennink-Kaminskia.h2kamins@unc.edu \\
\hline Notes & Clinical trials register: NCT0233-345 \\
\hline
\end{tabular}

Kobel 2017

Trial name or title Design, implementation, and study protocol of a kindergarten-based health promotion interven-
tion

\begin{tabular}{ll}
\hline Methods & Study design: Cluster-randomised longitudinal trial \\
\hline Participants & Service type: Kindergarten, children aged 3-6 years \\
& Region: Southwest Germany \\
& Number of services participating: 62 services \\
\hline Interventions & Number of experimental conditions: 2 (intervention, control) \\
& Policies, practices or programmes targeted by the intervention: \\
& Not specified \\
& Implementation strategies: Not specified \\
\hline Outcomes & $\begin{array}{l}\text { Outcome relating to the implementation of childcare service policies, practices or pro- } \\
\text { grammes: } \\
\text { - Change in environment of kindergarten }\end{array}$ \\
\hline
\end{tabular}


Kobel 2017 (Continued)

- Child nutrition intake (consumption of sugar-sweetened beverages, fruit, vegetables, high-calorie food)

\begin{tabular}{ll}
\hline Starting date & September 2016 \\
\hline Contact information & Susanne Kobel; susanne.kobel@uni-ulm.de \\
\hline Notes & $\begin{array}{l}\text { The study is registered at the German Clinical Trials Register (DRKS), Freiburg University, Germany, } \\
\text { ID: DRKS00010089. }\end{array}$ \\
\hline
\end{tabular}

Messiah 2016

\begin{tabular}{ll}
\hline Trial name or title & Healthy caregivers - healthy children (HC2) phase 2 \\
\hline Methods & Study design: randomised controlled trial \\
\hline Participants & Service type: Childcare centres \\
& Region: USA \\
& Number of services participating: 24 \\
\hline Interventions & Number of experimental conditions: 2 (intervention, control) \\
& Policies, practices or programmes targeted by the intervention: \\
& - implementation of the snack, screen time, physical activity, and beverage policies \\
& - Child health behaviours and parent and teacher health behaviours \\
& Implementation strategies: Not specified
\end{tabular}

\section{Outcomes}

\section{Outcome relating to the implementation of childcare service policies, practices or pro- grammes:}

- Change in centre nutrition and physical activity environment

- Change in centre menus

\begin{tabular}{ll}
\hline Starting date & 2015 \\
\hline Contact information & S.E. Messiah, smessiah@med.miami.edu \\
\hline Notes & Clinical Trials.gov number NCT02697565 \\
\hline
\end{tabular}

\section{NCT01890681}

\begin{tabular}{ll}
\hline Trial name or title & Baby NAPSACC intervention study \\
\hline Methods & Study design: randomised controlled trial \\
\hline Participants & Service type: childcare centres \\
& Region: North Carolina, USA \\
& Number of services participating: not specified \\
\hline \hline
\end{tabular}


NCT01890681 (Continued) Interventions
Number of experimental conditions: 2 (intervention, control)

Policies, practices or programmes targeted by the intervention: not specified Implementation strategies:

- Service and family self-assessment

- Targeted technical assistance provided by Baby NAPSACC consultant for providers and parents

- Training workshops for childcare providers

- Parent outreach and support
Outcome relating to the implementation of childcare service policies, practices or programmes: Change in childcare service policies and practices

\begin{tabular}{ll}
\hline Starting date & 2013 \\
\hline Contact information & Sara Benjamin Neelon, sara.benjamin@dm.duke.edu \\
\hline Notes & ClinicalTrials.gov Identifier: NCT01890681 \\
\hline
\end{tabular}

\section{NCT02375490} $\begin{array}{ll}\text { Trial name or title } & \text { A multilevel intervention to increase physical activity and improve healthy eating among young } \\ \text { children (ages } 3 \text { to 5) attending early childcare centres: the Healthy Start Study }\end{array}$

\begin{tabular}{|c|c|}
\hline Methods & Study design: randomised controlled trial \\
\hline \multirow[t]{3}{*}{ Participants } & Service type: early childcare centre \\
\hline & Region: Canada \\
\hline & Number of services participating: not specified \\
\hline \multirow[t]{10}{*}{ Interventions } & Number of experimental conditions: 2 (intervention, control) \\
\hline & Policies, practices or programmes targeted by the intervention: not specified \\
\hline & Implementation strategies: \\
\hline & $\begin{array}{l}\text { - Intersectoral partnerships that leads to promoting healthy weights in communities and childcare } \\
\text { services }\end{array}$ \\
\hline & - The Healthy Start guide for educators \\
\hline & - Customised training \\
\hline & - Role modelling and monitoring \\
\hline & $\begin{array}{l}\text { - An evidence-based resource for both families and educators and supplementary resources from } \\
\text { governmental partners }\end{array}$ \\
\hline & - Knowledge development and exchange \\
\hline & - Communication strategy \\
\hline Outcomes & $\begin{array}{l}\text { Outcome relating to the implementation of childcare service policies, practices or pro- } \\
\text { grammes: }\end{array}$ \\
\hline
\end{tabular}

Strategies to improve the implementation of healthy eating, physical activity and obesity prevention policies, practices or programmes 94 within childcare services (Review)

Copyright @ 2020 The Cochrane Collaboration. Published by John Wiley \& Sons, Ltd. 
NCT02375490 (Continued)

Early childcare centre practices and policies for physical activity and nutrition

\begin{tabular}{ll}
\hline Starting date & 2015 \\
\hline Contact information & Holly Hallikainen, hlh664@mail.usask.ca \\
\hline Notes & ClinicalTrials.gov Identifier: NCT02375490 \\
\hline
\end{tabular}

\section{NCT03075085}

Trial name or title care

\section{NCT03279926}

\begin{tabular}{ll}
\hline Trial name or title & Preschoolers Learning and Active in PlaY (PLAY) \\
\hline Methods & Study design: Randomised controlled trial \\
\hline Participants & Service type: Preschool \\
& Region: USA \\
& Number of services participating: not specified \\
\hline Interventions & Number of experimental conditions: 3 (all experimental) \\
& Policies, practices or programmes targeted by the intervention:
\end{tabular}

Strategies to improve the implementation of healthy eating, physical activity and obesity prevention policies, practices or programmes within childcare services (Review)

Copyright $\odot 2020$ The Cochrane Collaboration. Published by John Wiley \& Sons, Ltd.
Developing and testing implementation strategies for evidence-based obesity prevention in child-

pe: Head Start early childhood agencies

of services participating: not specified

Policies, practices or programmes targeted by the intervention:

Evidence-based obesity practices

Implementation strategies: Not specified

Outcome relating to the implementation of childcare service policies, practices or pro-

Change in educators' observed implementation fidelity scores for use of evidence-based obesity practices

January 2018

Taren Swindle, tswindle@uams.edu

ClinicalTrials.gov identifier: NCT03075085 
NCT03279926 (Continued)

- Active play opportunities, including teacher-led, child-initiated, outdoor and indoor

Implementation strategies:

Not specified

\begin{tabular}{ll}
\hline Outcomes & $\begin{array}{l}\text { Outcome relating to the implementation of childcare service policies, practices or pro- } \\
\text { grammes: Change in active play opportunities }\end{array}$ \\
\hline Starting date & September 2017 \\
\hline Contact information & Pooja Tandon, pooja.tandon@seattlechildrens.org \\
\hline Notes & ClinicalTrials.gov identifier: NCT03279926 \\
\hline
\end{tabular}

\section{NCT03590834}

\begin{tabular}{ll}
\hline Trial name or title & Míranos! Program, a preschool obesity prevention \\
\hline Methods & Study design: randomised controlled trial
\end{tabular}

\begin{tabular}{ll}
\hline Participants & Service type: Head Start childcare centres, aged 3-5 years \\
Region: USA & Number of services participating: 12 \\
\hline
\end{tabular}

Interventions Number of experimental conditions: 3 (centre-based, home-based, active control)

Policies, practices or programmes targeted by the intervention: Nutrition and physical activity policies and environments within the childcare centres

\section{Implementation strategies:}

- Nutrition and Physical activity policy modification to increase fruit and vegetable servings and more physical activity throughout the day.

- Staff training and assistance

- Health education and contests

Outcomes

Outcome relating to the implementation of childcare service policies, practices or programmes:

- Change in centre physical activity and nutrition PA policies and environments

\begin{tabular}{ll}
\hline Starting date & August 2018 \\
\hline Contact information & Vanessa Estrada, vanessa.estrada@utsa.edu \\
\hline Notes & ClinicalTrials.gov Identifier: NCT03590834 \\
\hline
\end{tabular}

Trial name or title PLAY (PhysicaL ActivitY) policy study


NCT03695523 (Continued) Methods

Participants

Study design: Randomised controlled trial

Service type: Childcare services

Region: London, Canada

Number of services participating: approximately 8

Number of experimental conditions: 2 (intervention, control)
Policies, practices or programmes targeted by the intervention:
- encouraging children to engage in higher intensity energetic play often
- aiming to accumulate 40 minutes each day
- exposing children to a variety of indoor and outdoor physical activities
- child-directed and teacher-facilitated active play daily
- short bouts of outdoor time for a total of 120 minutes each day made up of primarily unstructured
free play
- encouraging physical literacy by practicing fundamental movement skills
- not exposing children to screen-based technology during childcare
Implementation strategies: Not specified

\section{Outcomes Outcome relating to the implementation of childcare service policies, practices or pro- grammes:}

- Changes in children's sedentary time

- Changes in children's Moderate-to-Vigorous Physical Activity (MVPA)

- Environment and policy assessment and observation self-report

- Director environment and policy assessment and observation self-report

\begin{tabular}{ll}
\hline Starting date & October, 2018 \\
\hline Contact information & Trish Tucker, ttucker2@uwo.ca \\
\hline Notes & ClinicalTrials.gov Identifier: NCT03695523 \\
\hline
\end{tabular}

\section{Yoong 2016b}

$\begin{array}{ll}\text { Trial name or title } & \begin{array}{l}\text { A randomised controlled trial of an online menu planning intervention to improve childcare service } \\ \text { adherence to dietary guidelines }\end{array}\end{array}$

\begin{tabular}{ll}
\hline Methods & Study design: randomised controlled trial \\
\hline Participants & Service type: Childcare services (preschool and long daycare services) \\
& Region: New South Wales, Australia \\
& Number of services participating: 54 \\
\hline Interventions & Number of experimental conditions: 2 (intervention, control) \\
\hline
\end{tabular}

Strategies to improve the implementation of healthy eating, physical activity and obesity prevention policies, practices or programmes 
Policies, practices or programmes targeted by the intervention: Menu compliance with Caring for Children dietary guidelines

\section{Implementation strategies:}

- Web-based menu planning tool with decision support

- Face-to-face training and support to use the programme

- Provision of online resources

- Reminders

- Provision of portable computer tablets

- Communication strategies and managerial support

\begin{tabular}{ll}
\hline Outcomes & $\begin{array}{l}\text { Outcome relating to the implementation of childcare service policies, practices or pro- } \\
\text { grammes: } \\
\text { Mean number of food groups on childcare service menus that comply with dietary guidelines (Car- } \\
\text { ing for Children resource) regarding food provision to children in care }\end{array}$ \\
\hline Starting date & December 2016 \\
\hline Contact information & Dr Alice Grady, Alice.Grady@hnehealth.nsw.gov.au \\
\hline Notes & Australian New Zealand Clinical Trials Registry: ACTRN12616000974404 \\
\hline
\end{tabular}

EPAO: Environment and Policy Assessment and Observation

HC2: Healthy Caregivers Healthy Children

HMHW: Healthy Me, Healthy We

MVPA: Moderate to Vigorous Physical Activity

NAPSACC: Nutrition and Physical Activity Self-Assessment for Child Care

PA: Physical Activity

\section{DATA AND ANALYSES}

\section{Comparison 1. Implementation strategy versus usual care or waitlist control}

\begin{tabular}{|c|c|c|c|c|}
\hline Outcome or subgroup title & No. of studies & $\begin{array}{l}\text { No. of partici- } \\
\text { pants }\end{array}$ & Statistical method & Effect size \\
\hline 1 Implementation Score & 9 & 495 & $\begin{array}{l}\text { Std. Mean Difference (IV, Ran- } \\
\text { dom, 95\% CI) }\end{array}$ & $0.49[0.19,0.79]$ \\
\hline $\begin{array}{l}2 \text { Per cent of staff or services imple- } \\
\text { menting a policy or practice }\end{array}$ & 7 & & Odds Ratio (Random, 95\% Cl) & $1.83[0.81,4.11]$ \\
\hline
\end{tabular}


Analysis 1.1. Comparison 1 Implementation strategy versus usual care or waitlist control, Outcome 1 Implementation Score.

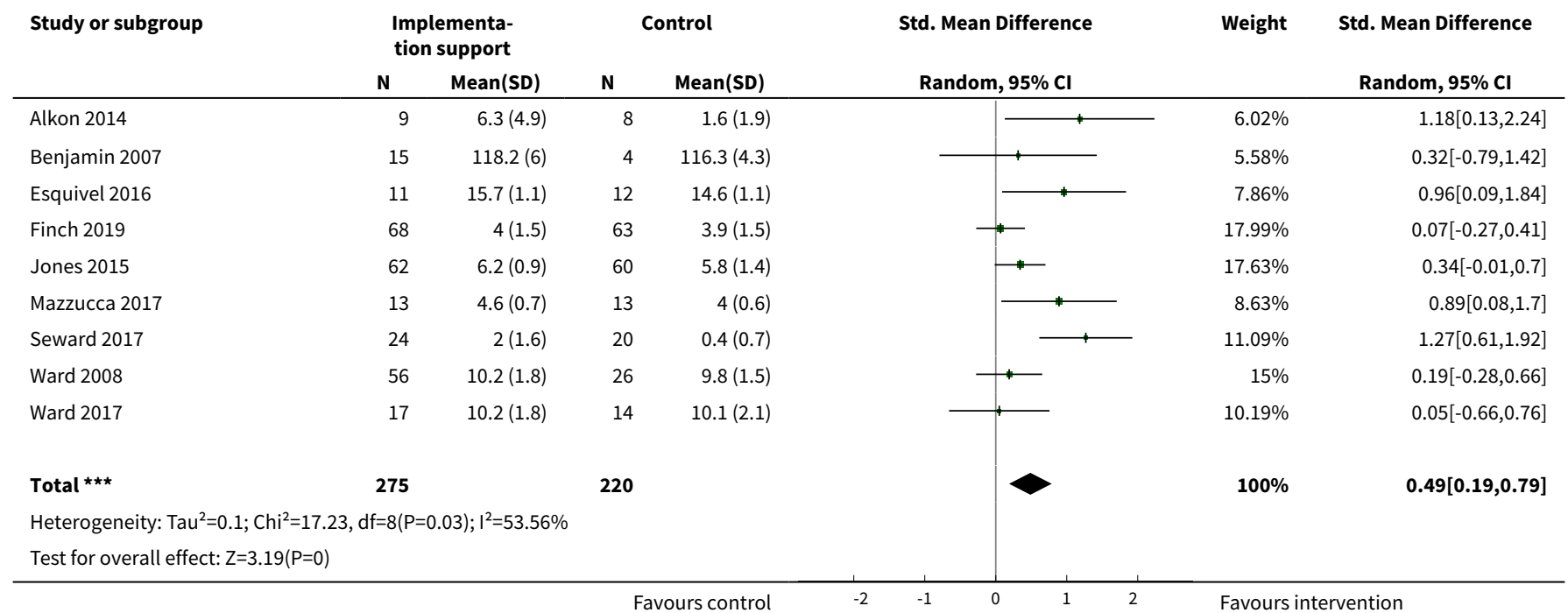

Analysis 1.2. Comparison 1 Implementation strategy versus usual care or waitlist control, Outcome 2 Per cent of staff or services implementing a policy or practice.

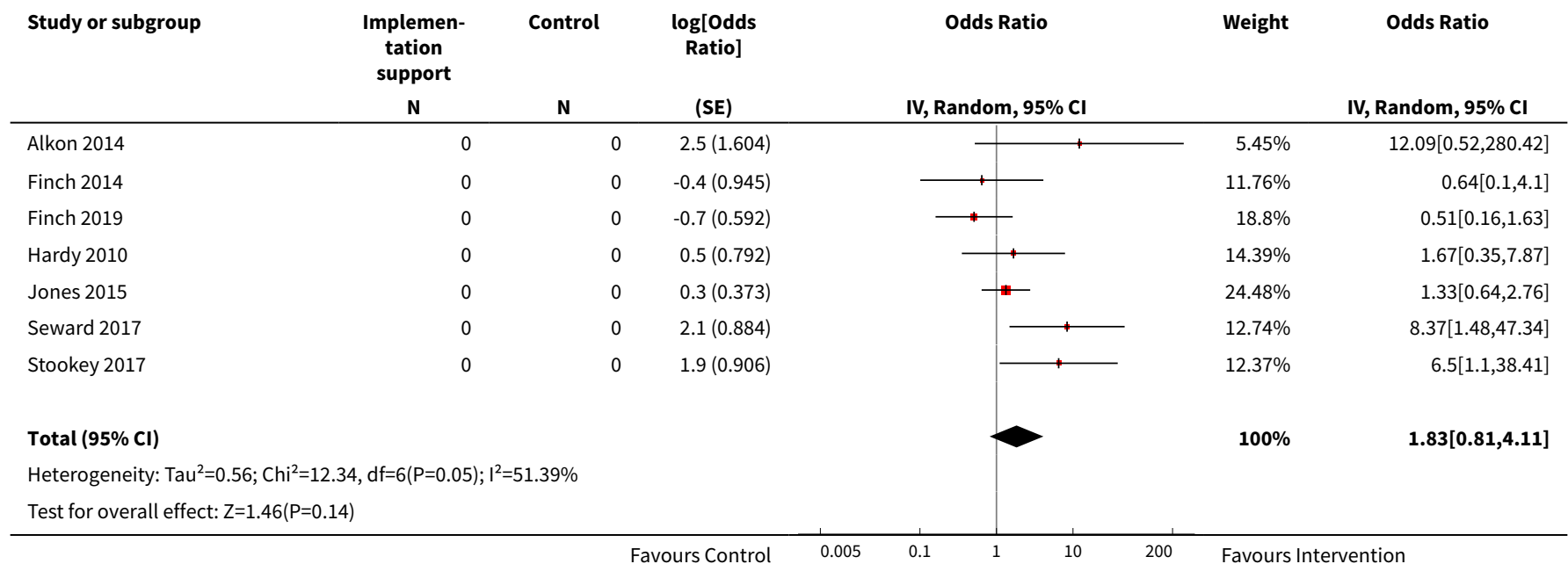

ADDITIONAL TABLES

Table 1. Summary of intervention, measures and absolute intervention effect size in included studies

\begin{tabular}{lllll}
\hline Study & $\begin{array}{l}\text { Implementation } \\
\text { strategies }\end{array}$ & $\begin{array}{l}\text { Comparison } \\
\text { group }\end{array}$ & $\begin{array}{l}\text { Primary implementation outcome mea- } \\
\text { sures }\end{array}$ & Effect size $\boldsymbol{a}$ \\
\hline Alkon 2014 & $\begin{array}{l}\text { Educational materials, } \\
\text { educational meetings } \\
\text { and audit and feed- } \\
\text { back }\end{array}$ & Usual practice & $\begin{array}{l}\text { Score: nutrition and physical activity policy } \\
\text { quality using the CHPHSPC and nutrition and } \\
\text { physical activity practices using the EPAO as- } \\
\text { sessed via observation (5 measures) }\end{array}$ & $\begin{array}{l}\text { Median } \\
\text { (range) }\end{array}$ \\
& & to 4.29$)$ & \\
\hline
\end{tabular}

Strategies to improve the implementation of healthy eating, physical activity and obesity prevention policies, practices or programmes 99 within childcare services (Review)

Copyright @ 2020 The Cochrane Collaboration. Published by John Wiley \& Sons, Ltd. 
Table 1. Summary of intervention, measures and absolute intervention effect size in included studies (Continued) $\%$ of staff or services implementing a practice: Services with a nutrition or physical activity policy ( 2 measures)

Median (range):

$\%$ of foods offered to children (10 measures)

$33 \% \%$ (22\% to $44 \%)^{c}$

Median (range): $7.7 \%(133 \%$ to

$-2.7 \%$

Bell $2014 \quad \begin{aligned} & \text { Educational materi- } \\ & \text { als, educational meet- }\end{aligned}$
$\begin{aligned} & \text { ings, audit and feed- } \\ & \text { back, opinion leaders, } \\ & \text { and small incentives } \\ & \text { or grants }\end{aligned}$

\section{$\%$ of staff or services implementing a prac- tice: percentage of services implementing nutrition policies and practices and menus consistent with nutrition recommendations (10 measures)}

Quantity of food served (servings/items): mean number of items or servings of healthy/ unhealthy foods on service menus (4 measures)
Median (range):

$9.5 \%$ (2\% to

$36 \%)$

Median (range):

0.5 serves/items $(-0.4$ to 0.8$)$
Score: nutrition, physical activity environments assessed via questionnaire (NAPSACC) completed by service managers (total score)
Mean difference

(95\% Cl)c: 5.10

$(-2.80$ to 13.00$)$ educational meetings, and audit and feedback

Educational materials, Usual practice educational meetings, audit and feedback, opinion leaders and small incentives

\author{
$\%$ of staff or services implementing a prac- \\ tice: percentage of services implementing \\ physical activity policies and practices (11 \\ measures) \\ Median (range): \\ $2.5 \%$ ( $-4 \%$ to \\ $41 \%)$
}

Minutes of service or staff implementation of a policy of practice: time (hours/ day) spent on structured physical activities (1 measure)
Mean: 6 minutes

\begin{tabular}{|c|c|}
\hline Finch 2014 & $\begin{array}{l}\text { Educational materials, } \\
\text { educational meetings, } \\
\text { audit and feedback, } \\
\text { opinion leaders and } \\
\text { small incentives }\end{array}$ \\
\hline
\end{tabular}
small incentives

Frequency of staff or service implementation of a practice: occasions of implementation of fundamental movement skill activities, staff role modelling and verbal prompts and positive comments (4 measures)
Minutes of service or staff implementation of a policy of practice (per session or day): minutes of fundamental movement skill ac- tivities, structured time, television viewing or seated time (4 measures)
$\%$ of staff or services implementing a prac- tice: services with seated time $>30$ minutes or with an activity policy ( 2 measures)

\section{Median (range): 2.6 (12.1 to 0.6 )}

\section{Median}

(range)c: 4.3

minutes $(-12$

minutes to 39

minutes)

Median (range): 5 (30 to -20$)$

Median (range): $-01(-0.6$ to -0.1$)$

\section{Mean number of resources or equipment} per service: ( 3 measures)

$\begin{array}{lllll}\text { Gosliner 2010 } & \text { Educational materi- } & \text { Educational ma- } & \% \text { of staff or services implementing a prac- } & \text { Median (range): } \\ & \text { als, educational meet- } & \text { terials, educa- } & \text { tice: Provision of food items by staff 'more of- } & 17 \%(0 \% \text { to } 23 \%) \\ & \text { ings, educational out- } & \text { tional meetings, } & \text { ten' assessed via staff-completed question- } & \\ & \begin{array}{ll}\text { reach visits or acad- } \\ \text { emic detailing with }\end{array} & \begin{array}{l}\text { educational out- } \\ \text { reach visits or }\end{array} & \text { naire (8 measures) } \\ & \text { small incentives or } & \text { academic detail- } & \\ & \text { ing } & \end{array}$

Strategies to improve the implementation of healthy eating, physical activity and obesity prevention policies, practices or programmes 100 within childcare services (Review)

Copyright @ 2020 The Cochrane Collaboration. Published by John Wiley \& Sons, Ltd. 
Table 1. Summary of intervention, measures and absolute intervention effect size in included studies (Continued) grants with staff wellness programme

\begin{tabular}{ll}
\hline Hardy $2010 \quad$ Educational materials, Usual practice \\
educational meetings, \\
educational outreach \\
visits or academic de- \\
tailing with small in- \\
centives or grants
\end{tabular}

\section{Minutes of service or staff implementation of a policy of practice: Minutes (per week or session) of structured and unstructured play or fundamental movement skills activities ( 3 measures)}

Frequency of staff or service implementation of a practice: Frequency (per week or day) of structured or unstructured play, and of fundamental movement skill activities (3 measures)

$\%$ of staff or services implementing a practice: conduct of food-based activities, development of new rules around food and drink bought from home, and the provision of health information to families ( 3 measures)

\begin{tabular}{lll}
\hline $\begin{array}{l}\text { Johnston Molloy } \\
2013\end{array}$ & $\begin{array}{l}\text { Educational materi- } \\
\text { als, manager and staff } \\
\text { educational meetings } \\
\text { and audit and feed- } \\
\text { back }\end{array}$ & $\begin{array}{l}\text { Educational ma- } \\
\text { terials, manag- } \\
\text { er educational } \\
\text { meetings, and } \\
\text { audit and feed- } \\
\text { back }\end{array}$ \\
\hline Ward 2008 & $\begin{array}{ll}\text { Educational materials, } \\
\text { educational meetings, } \\
\text { and audit and feed- } \\
\text { back }\end{array}$ & Usual practice \\
\hline Williams 2002 & $\begin{array}{l}\text { Educational materials, } \\
\text { educational meetings, } \\
\text { educational outreach } \\
\text { visits or academic de- }\end{array}$ & \\
& $\begin{array}{l}\text { tailing with small in- } \\
\text { centives or grants }\end{array}$ & \\
\hline
\end{tabular}

\begin{tabular}{lll}
$\begin{array}{l}\text { Johnston Molloy } \\
2013\end{array}$ & $\begin{array}{l}\text { Educational materi- } \\
\text { als, manager and staff } \\
\text { educational meetings } \\
\text { and audit and feed- } \\
\text { back }\end{array}$ & $\begin{array}{l}\text { Educational ma- } \\
\text { terials, manag- } \\
\text { er educational } \\
\text { meetings, and } \\
\text { audit and feed- } \\
\text { back }\end{array}$ \\
\hline Ward 2008 & $\begin{array}{ll}\text { Educational materials, } \\
\text { educational meetings, } \\
\text { and audit and feed- } \\
\text { back }\end{array}$ & \\
\hline Williams 2002 & $\begin{array}{l}\text { Educational materials, } \\
\text { educational meetings, } \\
\text { educational outreach } \\
\text { visits or academic de- }\end{array}$ & \\
& $\begin{array}{l}\text { Uailing with small in- } \\
\text { centives or grants }\end{array}$ &
\end{tabular}
educational meetings, and audit and feedback

Score: On the Health Promotion Evaluation Activity Scored Evaluation form assessed via observation (total score)

Difference in median score: $-2^{b}$

Score: nutrition and physical activity prac-

Mean difference tices using the EPAO assessed via observation (total score) $(95 \% \mathrm{CI}) \mathrm{c}^{\mathrm{i}} 1.01$ (0.18 to 1.84$)$
$0.2(-0.9$ to 1.9$)$

\section{Median}

(range)c: $11 \%$

$(-7 \%$ to $31 \%)$
Quantity of food served (servings/grams): Primary outcome - grams of saturated fat assessed via menu audit (one measure)
Median (range):

$17 \%(0 \%$ to $23 \%)$
Median (range):

7.7 minutes $(6.5$ minutes to 10.1

minutes

(

\begin{tabular}{ll}
\hline O'Neill $2017 \quad \begin{array}{l}\text { Educational meetings, Usual practice } \\
\text { release of practice } \\
\text { guidelines }\end{array}$
\end{tabular}

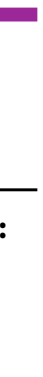


Table 1. Summary of intervention, measures and absolute intervention effect size in included studies (Continued) and implementation support staff

\begin{tabular}{ll}
\hline Seward $2017 \quad$ Audit with feedback, \\
& educational materials, \\
& educational meetings, \\
& opinion leaders, edu- \\
& cational outreach or \\
& academic detailing
\end{tabular}

Usual practice educational materials, educational meetings, cational outreach or academic detailing
Score: Mean number of food groups compliant with guidelines

$\%$ of staff or services implementing a prac-

tice: Proportion of services fully compliant with nutrition guidelines for all food groups ( 7 measures)

Mean difference

$1.57(0.82,2.33)$

OR Median (range):

OR $6.26 ; 95 \% \mathrm{Cl}$

1.26 to 43.40 (OR

1 to 16.54$)$

Yoong $2016 \quad$ Educational materials Usual practice

Quantity of food served: number of fruit and vegetable serves on the service menu assessed via questionnaire (2 measures)

Mean difference median (range): 0.25 (0.0 to 0.50$)$

Esquivel 2016

Educational materials, educational meetings. Other: monthly employee wellness activities
Waiting-list control (delayed intervention)
Score: nutrition and physical activity environments using the EPAO assessed via researcher observation (total score)
Mean difference: 1.1

\begin{tabular}{|c|c|}
\hline Mazzucca 2017 & $\begin{array}{l}\text { Educational materials, } \\
\text { educational meetings, } \\
\text { educational outreach } \\
\text { or academic detailing, } \\
\text { reminders, tailored in- } \\
\text { terventions }\end{array}$ \\
\hline
\end{tabular}

Score: physical activity environment assessed using modified EPAO assessed via service self-report (7 measures)

\section{Median/Mean} differences (range): $0.4(-0.7$ to 0.9$)$

\section{Morshed $2016 \quad$ Educational materials, Usual practice educational meeting}

\begin{abstract}
Quantity of food served: number of fruit, vegetable, and whole grain servings, grams of discretionary fat, teaspoons of added sugar, and grams of fat from milk provided assessed via researcher observation (6 measures)
\end{abstract}

\section{Relative change estimate:}

OR $(95 \% \mathrm{Cl}): 1.00$

(0.81 to 1.24$)$ (OR

1.09 to 0.82 )

Mean difference: 15.17

$\begin{array}{llll}\text { Sharma } 2018 & \begin{array}{l}\text { Educational meetings, } \\ \text { educational outreach } \\ \text { or academic detailing, } \\ \text { reminders }\end{array} & \begin{array}{l}\text { Score: Implementation index assessed via } \\ \text { teacher-completed survey (1 measure) }\end{array} & \begin{array}{l}\text { Mean differ- } \\ \text { ence: } 15.17\end{array} \\ \end{array}$

Stookey 2017
Audit with feedback, educational materials, educational meetings, educational outreach or academic detailing, incentives, tailored in- terventions

Waiting-list control (delayed intervention).
$\%$ of staff or services implementing a prac- tice: children's exposure to three nutrition and physical activity practices: use of physical activity curriculum, staff usually join in phys- ically active play; pitchers of drinking water visible in the classroom (1 measure)

OR (95\% Cl): 6.5 ; (1.1 to 40.6)

\begin{tabular}{lllll}
\hline Ward 2017 & $\begin{array}{l}\text { Audit with feedback, } \\
\text { educational materials, } \\
\text { educational meetings }\end{array}$ & $\begin{array}{l}\text { Waiting-list con- } \\
\text { trol (delayed in- } \\
\text { tervention) }\end{array}$ & $\begin{array}{l}\text { Score: nutrition environment assessed using } \\
\text { the EPAO assessed via service self-report (to- } \\
\text { tal score) }\end{array}$ & $\begin{array}{l}\text { Mean differ- } \\
\text { ence: } 0.73\end{array}$ \\
\hline Finch 2019 & $\begin{array}{l}\text { Educational materials, } \\
\text { audit with feedback, } \\
\text { continuous quality im- } \\
\begin{array}{l}\text { provement, educa- } \\
\text { tional outreach or aca- }\end{array}\end{array}$ & Usual practice & $\begin{array}{l}\text { Score: mean number of policies and practices } \\
\text { implemented (1 measure) }\end{array}$ & $\begin{array}{l}\text { Mean differ- } \\
\text { ence: } 0.1 ; 95 \% \mathrm{Cl}\end{array}$ \\
& & $\begin{array}{l}\text { \% of staff or services implementing a prac- } \\
\text { tice: Proportion of services implementing all } \\
\text { six policies/practices (1 measure) }\end{array}$ & $\begin{array}{l}\text { OR (95\% } \mathbf{C l}): 0.51 \\
(0.16 \text { to } 1.58)\end{array}$ \\
\hline
\end{tabular}

Strategies to improve the implementation of healthy eating, physical activity and obesity prevention policies, practices or programmes 102 within childcare services (Review)

Copyright $\odot 2020$ The Cochrane Collaboration. Published by John Wiley \& Sons, Ltd. 
Table 1. Summary of intervention, measures and absolute intervention effect size in included studies (Continued) demic detailing, opin-

ion leaders, tailored

interventions

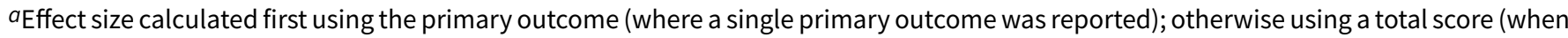
total and subscale scores were provided); otherwise using the median effect size across measures (where more than one outcome measure was reported and not specified as primary).

bMean not reported. Represents the difference in median score between manager- and staff-trained versus manager only-trained group.

cAdditional data obtained from study authors where unclear or missing

CHPHSPC: Californian Childcare Health Programme Health and Safety Checklist

DOCC: Diet Observation in Child Care

EPAO: Environment and Policy Assessment and Observation

NAPSACC: Nutrition and Physical Activity Self-Assessment for Child Care

Table 2. Definition of EPOC subcategories utilised in the review

\begin{tabular}{ll}
\hline EPOC subcategory & Definition \\
\hline Educational materials & $\begin{array}{l}\text { Distribution to individuals, or groups, of educational materials to support clinical care, i.e. any in- } \\
\text { tervention in which knowledge is distributed. For example, this may be facilitated by the internet, } \\
\text { learning critical appraisal skills; skills for electronic retrieval of information, diagnostic formula- } \\
\text { tion; question formulation. }\end{array}$ \\
\hline
\end{tabular}

Educational meetings Courses, workshops, conferences or other educational meetings
$\begin{array}{ll}\begin{array}{l}\text { Educational outreach visits or } \\ \text { academic detailing }\end{array} & \begin{array}{l}\text { Personal visits by a trained person to health workers in their own settings, to provide information } \\ \text { with the aim of changing practice }\end{array}\end{array}$

Small incentives or grants

Transfer of money or material goods to healthcare providers conditional on taking a measurable action or achieving a predetermined performance target, for example incentives for lay health workers

Audit and feedback $\quad \begin{aligned} & \text { A summary of health workers' performance over a specified period of time, given to them in a writ- } \\ & \text { ten, electronic or verbal format; the summary may include recommendations for clinical action. }\end{aligned}$

Opinion leaders

The identification and use of identifiable local opinion leaders to promote good clinical practice

Tailored interventions Interventions to change practice that are selected based on an assessment of barriers to change, for example, through interviews or surveys.

Meminders
consultation with a patient, for example, computer decision support systems

Local opinion leaders The identification and use of identifiable local opinion leaders to promote good clinical practice

Local consensus processes

Formal or informal local consensus processes, for example, agreeing a clinical protocol to manage a patient group, adapting a guideline for a local health system or promoting the implementation of guidelines

Clinical Practice Guidelines tients to decide on appropriate healthcare for specific clinical circumstances (USA IOM).

IOM: Institute of Medicine 


\section{A P P E N D I CES}

\section{Appendix 1. Search strategy}

\section{MEDLINE search strategy}

1. exp obesity/

2. Weight Gain/

3. exp Weight Loss/

4. obes ${ }^{\star} . \mathrm{mp}$.

5. (weight gain or weight loss).mp.

6. (overweight or over weight or overeat* or over eat ${ }^{\star}$ ).mp.

7. weight change ${ }^{\star} . \mathrm{mp}$.

8. ((bmi or body mass index) adj2 (gain or loss or change)).mp.

9. 1 or 2 or 3 or 4 or 5 or 6 or 7 or 8

10. exp Exercise/

11. physical inactivity.mp.

12. physical activity.mp.

13. Motor Activity/

14. (physical education or physical training).mp. [mp=title, abstract, original title, name of substance word, subject heading word, floating sub-heading word, keyword heading word, organism supplementary concept word, protocol supplementary concept word, rare disease supplementary concept word, unique identifier, synonyms]

15. "Physical Education and Training"/

16. Physical Fitness/

17. sedentary.mp.

18. exp Life Style/

19. exp Leisure Activities/

20. Dancing/

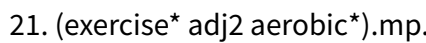

22. sport*.mp.

23. ((lifestyle or life style) adj5 activ*).mp.

24. (dance* or dancing).mp.

25.10 or 11 or 12 or 13 or 14 or 15 or 16 or 17 or 18 or 19 or 20 or 21 or 22 or 23 or 24

26. exp Diet/

27. nutrition ${ }^{\star} . \mathrm{mp}$.

28. (health ${ }^{\star}$ adj2 eat*).mp.

29. Child Nutrition Sciences/

30. exp Fruit/ or fruit*.mp.

31. Vegetables/ or vegetable*.mp.

Strategies to improve the implementation of healthy eating, physical activity and obesity prevention policies, practices or programmes

Copyright $\odot 2020$ The Cochrane Collaboration. Published by John Wiley \& Sons, Ltd. 
32. "Fruit and Vegetable Juices"/

33. canteen*.mp.

34. Food Services/

35. menu*.mp.

36. (calorie or calories or kilojoule ${ }^{\star}$ ).mp.

37. energy density.mp.

38. Eating/

39. Feeding Behavior/ or feeding behavio? ${ }^{\star}{ }^{\star} . \mathrm{mp}$.

40. dietary intake.mp.

41. Food Habits/

42. Food/

43. Carbonated Beverages/ or soft drink ${ }^{\star} . \mathrm{mp}$.

44. soda.mp.

45. sweetened drink ${ }^{\star} . \mathrm{mp}$.

46. Dietary Fats/

47. confectionary.mp.

\left.\left. 48. ( ${\text { school adj2 }\left(\text { lunch }^{\star} \text { or meal }\right.}^{\star}\right)\right) \cdot \mathrm{mp}$.

49. Menu Planning/

50. feeding program ${ }^{\star} . \mathrm{mp}$.

51. food program.mp.

52. (nutrition ${ }^{\star}$ adj2 program $\left.{ }^{\star}\right) \cdot \mathrm{mp}$.

53. cafeteria*.mp.

54. Nutritional Status/

55.26 or 27 or 28 or 29 or 30 or 31 or 32 or 33 or 34 or 35 or 36 or 37 or 38 or 39 or 40 or 41 or 42 or 43 or 44 or 45 or 46 or 47 or 48 or 49 or 50 or 51 or 52 or 53 or 54

56.9 or 25 or 55

57. Child, Preschool/

58. $\left(\right.$ pre-school $^{\star}$ or preschool $\left.^{\star}\right) \cdot \mathrm{mp}$.

59. Child Day Care Centers/

60. (childcare* or child care $\left.{ }^{\star}\right) \cdot \mathrm{mp}$.

61. (daycare* or day care $\left.{ }^{\star}\right) \cdot \mathrm{mp}$.

62. early child*.mp.

63. (nursery or nurseries).mp. [mp=title, abstract, original title, name of substance word, subject heading word, floating sub-heading word, keyword heading word, organism supplementary concept word, protocol supplementary concept word, rare disease supplementary concept word, unique identifier, synonyms]

64. Kinder*.mp.

Strategies to improve the implementation of healthy eating, physical activity and obesity prevention policies, practices or programmes

Copyright (c) 2020 The Cochrane Collaboration. Published by John Wiley \& Sons, Ltd. 
65.57 or 58 or 59 or 60 or 61 or 62 or 63 or 64

66. randomized controlled trial.pt.

67. controlled clinical trial.pt.

68. clinical trials as topic.sh.

69. exp Cohort studies/

70. Controlled Before-After Studies/

71. Interrupted Time Series Analysis/

72. comparative study.pt.

73. trial ${ }^{\star}$.tw.

74. double blind.tw.

75. single blind.tw.

76. experiment ${ }^{\star}$. tw.

77. (pretest or pre test).tw.

78. (posttest or post test).tw.

79. (pre post or prepost).tw.

80. before after.tw.

81. qua?i randomi?ed.tw.

82. stepped wedge.tw.

83. (non randomi?ed or nonrandomi?ed).tw.

84. interrupted time series.tw.

85. multiple baseline.tw.

86. regression discontinuity.tw.

87. comprehensive cohort.tw.

88. random ${ }^{\star}$.ab.

89.66 or 67 or 68 or 69 or 70 or 71 or 72 or 73 or 74 or 75 or 76 or 77 or 78 or 79 or 80 or 81 or 82 or 83 or 84 or 85 or 86 or 87 or 88 90. implement*.mp.

91. Health Promotion/mt [Methods]

92. "Outcome and Process Assessment (Health Care)"/

93. "Process Assessment (Health Care)"/

94. "Outcome Assessment (Health Care)"/

95. Program Evaluation/

96. Interrupted Time Series Analysis/

97. dissemin ${ }^{\star} . \mathrm{mp}$.

98. adopt*.mp.

99. practice*.mp.

Strategies to improve the implementation of healthy eating, physical activity and obesity prevention policies, practices or programmes

Copyright @ 2020 The Cochrane Collaboration. Published by John Wiley \& Sons, Ltd. 
100. organi?ational change ${ }^{\star} . \mathrm{mp}$.

101. diffus*.mp.

102. (system* adj2 change*).tw.

103. quality improvement ${ }^{\star} . \mathrm{mp}$.

104. transform ${ }^{\star} . \mathrm{mp}$.

105. translat*.mp.

106. transfer*.mp.

107. uptake*.mp.

108. sustainab*.mp.

109. institutionali*.mp.

110. routin*.mp.

111. maintenance.mp.

112. capacity.mp.

113. incorporat ${ }^{\star} . \mathrm{mp}$.

114. adher*.mp.

115. ((polic ${ }^{\star}$ or practice or program $^{\star}$ or innovation ${ }^{\star}$ ) adj5 (performance or feedback or prompt ${ }^{\star}$ or reminder ${ }^{\star}$ or incentive* or penalt ${ }^{\star}$ or communicat $^{\star}$ or social market* or professional development or network ${ }^{\star}$ or leadership or opinion leader ${ }^{\star}$ or consensus process ${ }^{\star}$ or change manage* or train* or audit*)).mp.

116. integrat*.mp.

117. scal* up.mp.

118.90 or 91 or 92 or 93 or 94 or 95 or 97 or 98 or 99 or 100 or 101 or 102 or 103 or 104 or 105 or 106 or 107 or 108 or 109 or 110 or 111 or 112 or 113 or 114 or 115 or 116 or 117

119. 56 and 65 and 89 and 118

\section{CENTRAL search strategy}

1. MeSH descriptor: [Obesity] explode all trees

2. MeSH descriptor: [Weight Gain] this term only

3. MeSH descriptor: [Weight Loss] explode all trees

4. obes*:ti,ab,kw

5. ("weight gain" or "weight loss"):ti,ab,kw

6. (overweight or "over weight" or overeat* or "over eat"):ti,ab,kw

7. "weight change ${ }^{\star "}: \mathrm{ti}, \mathrm{ab}, \mathrm{kw}$

8. ((bmi or "body mass index") near/2 (gain or loss or change)):ti,ab,kw

9. $\{$ or \#1-\#8\}

10. MeSH descriptor: [Exercise] explode all trees

11. "physical inactivity":ti,ab,kw

12. "physical activity":ti,ab,kw

Strategies to improve the implementation of healthy eating, physical activity and obesity prevention policies, practices or programmes

Copyright (c) 2020 The Cochrane Collaboration. Published by John Wiley \& Sons, Ltd. 
13. MeSH descriptor: [Motor Activity] this term only

14. ("physical education" or "physical training"):ti,ab,kw

15. MeSH descriptor: [Physical Education and Training] explode all trees

16. MeSH descriptor: [Physical Fitness] this term only

17. sedentary:ti,ab,kw

18. MeSH descriptor: [Life Style] explode all trees

19. MeSH descriptor: [Leisure Activities] explode all trees

20. MeSH descriptor: [Dancing] this term only

21. (exercis* near/2 aerobic*):ti,ab,kw

22. sport*:ti,ab,kw

23. (("life style" or lifestyle) near/5 activ^):ti,ab,kw

24. (dance ${ }^{\star}$ or dancing):ti,ab,kw

25. $\{$ or \#10-\#24\}

26. MeSH descriptor: [Diet] explode all trees

27. nutrition*:ti,ab,kw

28. (health* near/2 eat $\left.{ }^{\star}\right): t i, a b, k w$

29. MeSH descriptor: [Child Nutrition Sciences] this term only

30. fruit* :ti,ab,kw

31. MeSH descriptor: [Fruit] this term only

32. vegetable*:ti,ab,kw

33. MeSH descriptor: [Vegetables] this term only

34. canteen*:ti,ab,kw

35. MeSH descriptor: [Fruit and Vegetable Juices] this term only

36. MeSH descriptor: [Food Services] this term only

37. menu*:ti,ab,kw

38. (calorie or calories or kilojoule $\left.{ }^{\star}\right)$ :ti,ab,kw

39. "energy density":ti,ab,kw

40. MeSH descriptor: [Eating] this term only

41. MeSH descriptor: [Feeding Behavior] this term only

42. "feeding behavio*":ti,ab,kw

43. "dietary intake":ti,ab,kw

44. MeSH descriptor: [Food] this term only

45. MeSH descriptor: [Carbonated Beverages] this term only

46. "soft drink*":ti,ab,kw

47. soda:ti,ab,kw

Strategies to improve the implementation of healthy eating, physical activity and obesity prevention policies, practices or programmes 
48. "sweetened drink*":ti,ab,kw

49. MeSH descriptor: [Dietary Fats] this term only

50. confectionary:ti,ab,kw

51. (school near/2 (lunch* or meal $\left.\left.{ }^{\star}\right)\right): t i, a b, k w$

52. MeSH descriptor: [Menu Planning] this term only

53. "feeding program*":ti,ab,kw

54. "food program*":ti,ab,kw

55. (nutrition* near/2 program ${ }^{\star}$ ):ti,ab,kw

56. cafeteria*:ti,ab,kw

57. MeSH descriptor: [Nutritional Status] this term only

58. $\{$ or \#26-\#57\}

59. \{or \#1-\#57\}

60. MeSH descriptor: [Child, Preschool] this term only

61. ("pre-school*" or preschool $\left.{ }^{\star}\right): t i, a b, k w$

62. MeSH descriptor: [Child Day Care Centers] this term only

63. (childcare* or "child care*"):ti,ab,kw

64. (daycare* or "day care*"):ti,ab,kw

65. "early child*":ti,ab,kw

66. (nursery or nurseries):ti,ab,kw

67. Kinder*:ti,ab,kw

68. $\{$ or \#60-\#67\}

69. implement*:ti,ab,kw

70. dissemin*:ti,ab,kw

71. adopt*:ti,ab,kw

72. practice $^{\star}: \mathrm{ti}, \mathrm{ab}, \mathrm{kw}$

73. "organi?ational change*":ti,ab,kw

74. diffus*:ti,ab,kw

75. system ${ }^{\star}$ near/2 change* $:$ ti,ab,kw

76. "quality improvement ${ }^{\star} ": t i, a b, k w$

77. transform*:ti,ab,kw

78. translat*:ti,ab,kw

79. transfer*:ti,ab,kw

80. uptake*:ti,ab,kw

81. sustainab*:ti,ab,kw

82. institutionali*:ti,ab,kw

Strategies to improve the implementation of healthy eating, physical activity and obesity prevention policies, practices or programmes 
83. routin*:ti,ab,kw

84. maintenance:ti,ab,kw

85. capacity:ti,ab,kw

86. incorporat*:ti,ab,kw

87. adher*:ti,ab,kw

88. ((polic ${ }^{\star}$ or practice ${ }^{\star}$ or program* or innovation*) near/5 (performance or feedback or prompt* or reminder ${ }^{\star}$ or incentive* or penalt ${ }^{\star}$ or communicat ${ }^{\star}$ or social market* or professional development or network ${ }^{\star}$ or leadership or opinion leader ${ }^{\star}$ or consensus process ${ }^{\star}$ or change manage* or train* or audit*)):ti,ab,kw

89. integrat*:ti,ab,kw

90. "scal* up":ti,ab,kw

91. MeSH descriptor: [Health Promotion] this term only and with qualifier(s): [methods - MT]

92.MeSH descriptor: [Outcome and Process Assessment (Health Care)] this term only

93. MeSH descriptor: [Process Assessment (Health Care)] this term only

94. MeSH descriptor: [Outcome Assessment (Health Care)] this term only

95. MeSH descriptor: [Program Evaluation] this term only

96. \{or \#69-\#95\}

97. \{and \#59, \#68, \#96\} with Publication Year from 2016 to 2019, in Trials

\section{Embase search strategy}

1. exp obesity/

2. weight gain/

3. Weight Loss.mp. or exp weight reduction/

4. obes*.mp.

5. (weight gain or weight loss).mp.

6. (overweight or over weight or overeat* or over eat ${ }^{\star}$ ).mp.

7. weight change*.mp.

8. ((bmi or body mass index) adj2 (gain or loss or change)).mp.

9. 1 or 2 or 3 or 4 or 5 or 6 or 7 or 8

10. exp exercise/

11. physical inactivity.mp. or physical inactivity/

12. exp physical activity/

13. exp motor activity/

14. (physical education or physical training).mp.

15. physical education/

16. physical fitness.mp. or fitness/

17. sedentary.mp.

18. lifestyle/

Strategies to improve the implementation of healthy eating, physical activity and obesity prevention policies, practices or programmes 
19. Leisure Activities.mp. or leisure/

20. exp sport/

21. dancing/

22. (exercise ${ }^{\star}$ adj2 aerobic $\left.{ }^{\star}\right) \cdot \mathrm{mp}$.

23. sport $^{\star} . \mathrm{mp}$.

24. ((lifestyle or life style) adj5 $\left.\operatorname{activ}^{\star}\right)$.mp.

25. (dance* or dancing).mp.

26. 10 or 11 or 12 or 13 or 14 or 15 or 16 or 17 or 18 or 19 or 20 or 21 or 22 or 23 or 24 or 25

27. exp diet/

28. nutrition*.mp. or nutrition/

29. (health* adj2 eat $\left.{ }^{\star}\right) \cdot m p$.

30. Child Nutrition Sciences.mp. or nutritional science/

31. fruit*.mp. or fruit/ or "fruit and vegetable juice"/

32. vegetable*.mp. or vegetable/

33. canteen*.mp.

34. Food Services.mp. or catering service/

35. Menu*.mp.

36. (calorie or calories or kilojoule*).mp.

37. Energy Intake.mp. or caloric intake/

38. energy density.mp.

39. eating/

40. feeding behavio? ${ }^{\star}$.mp. or feeding behavior/

41. dietary intake.mp. or dietary intake/

42. Food Habit*.mp.

43. food/

44. carbonated beverage/ or soft drink ${ }^{\star}$.mp. or soft drink/

45. soda.mp.

46. sweetened drink ${ }^{\star} . \mathrm{mp}$.

47. Dietary Fats.mp. or fat intake/

48. confectionary.mp.

49. (school adj2 (lunch* or meal $\left.\left.{ }^{\star}\right)\right) \cdot \mathrm{mp}$.

50. Menu Planning.mp.

51. feeding program $^{\star} . \mathrm{mp}$.

52. food program.mp.

53. (nutrition* adj2 program*).mp.

Strategies to improve the implementation of healthy eating, physical activity and obesity prevention policies, practices or programmes 
54. cafeteria*.mp.

55. nutritional status/

56.27 or 28 or 29 or 30 or 31 or 32 or 33 or 34 or 35 or 36 or 37 or 38 or 39 or 40 or 41 or 42 or 43 or 44 or 45 or 46 or 47 or 48 or 49 or 50 or 51 or 52 or 53 or 54 or 55

57.9 or 26 or 56

58. Child, Preschool/

59. $\left(\right.$ pre-school $^{\star}$ or preschool $\left.{ }^{\star}\right) \cdot \mathrm{mp}$.

60. day care/

61. child care/ or childcare ${ }^{\star} . \mathrm{mp}$.

62. (daycare* or day care*).mp.

63. early child ${ }^{\star} . \mathrm{mp}$.

64. nurseries.mp. or nursery/

65. Kinder*.mp.

66.58 or 59 or 60 or 61 or 62 or 63 or 64 or 65

67. randomized controlled trial/

68. controlled clinical trial/

69. "clinical trial (topic)"/

70. trial ${ }^{*}$.tw.

71. double blind.tw.

72. single blind.tw.

73. experiment ${ }^{\star}$.tw.

74. (pretest or pre test).tw.

75. (posttest or post test).tw.

76. (pre post or prepost).tw.

77. before after.tw.

78. qua?i randomi?ed.tw.

79. stepped wedge.tw.

80. (non randomi?ed or nonrandomi?ed).tw.

81. interrupted time series.tw.

82. multiple baseline.tw.

83. regression discontinuity.tw.

84. comprehensive cohort.tw.

85. random*.ab.

86. cohort analysis/

87.67 or 68 or 69 or 70 or 71 or 72 or 73 or 74 or 75 or 76 or 77 or 78 or 79 or 80 or 81 or 82 or 83 or 84 or 85 or 86

Strategies to improve the implementation of healthy eating, physical activity and obesity prevention policies, practices or programmes

Copyright @ 2020 The Cochrane Collaboration. Published by John Wiley \& Sons, Ltd. 
88. implement ${ }^{\star} . \mathrm{mp}$

89. dissemin ${ }^{\star} . \mathrm{mp}$.

90. adopt* ${ }^{\star} . \mathrm{mp}$.

91. organi?ational change ${ }^{\star} . \mathrm{mp}$.

92. diffus ${ }^{\star} . \mathrm{mp}$.

93. (system ${ }^{\star}$ adj2 change*).tw.

94. quality improvement ${ }^{\star}$.mp.

95. practice $^{\star} . \mathrm{mp}$.

96. transform ${ }^{\star} \cdot \mathrm{mp}$.

97. translat* ${ }^{\star} . \mathrm{mp}$.

98. transfer ${ }^{\star} . \mathrm{mp}$.

99. uptake*.mp.

100. sustainab*.mp.

101. institutionali ${ }^{\star} . \mathrm{mp}$.

102. routin*.mp.

103. maintenance.mp.

104. capacity.mp.

105. incorporat ${ }^{\star} . \mathrm{mp}$.

106. adher $^{\star} . \mathrm{mp}$.

107. ((polic ${ }^{\star}$ or practice ${ }^{\star}$ or program ${ }^{\star}$ or innovation ${ }^{\star}$ ) adj5 (performance or feedback or prompt ${ }^{\star}$ or reminder ${ }^{\star}$ or incentive or penalt $^{\star}$ or communicat $^{\star}$ or social market ${ }^{\star}$ or professional development or network ${ }^{\star}$ or leadership or opinion leader ${ }^{\star}$ or consensus process ${ }^{\star}$ or change manage* or train* or audit*)).mp.

108. integrat ${ }^{\star} . \mathrm{mp}$.

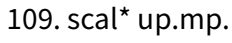

110. health care quality/

111. quality control/

112. program evaluation/

113. total quality management/

114. 88 or 89 or 90 or 91 or 92 or 93 or 94 or 95 or 96 or 97 or 98 or 99 or 100 or 101 or 102 or 103 or 104 or 105 or 106 or 107 or 108 or 109 or 110 or 111 or 112 or 113

115. 57 and 66 and 87 and 114

\section{PsychINFO search strategy}

1. Obesity/

2. Weight Gain/

3. Weight Loss/

4.obes ${ }^{\star} . \mathrm{mp}$.

Strategies to improve the implementation of healthy eating, physical activity and obesity prevention policies, practices or programmes

Copyright $\odot 2020$ The Cochrane Collaboration. Published by John Wiley \& Sons, Ltd. 
5. (weight gain or weight loss).mp.

6. (overweight or over weight or overeat* or over eat ${ }^{\star}$ ).mp.

7. weight change ${ }^{\star} \cdot \mathrm{mp}$.

8. ((bmi or body mass index) adj2 (gain or loss or change)).mp.

9. 1 or 2 or 3 or 4 or 5 or 6 or 7 or 8

10. exp Exercise/

11. physical inactivity.mp.

12. physical activity.mp. or Physical Activity/

13. Motor Activity.mp.

14. (physical education or physical training).mp.

15. Physical Education/

16. Physical Fitness/

17. sedentary.mp.

18. exp Lifestyle/

19. leisure time/ or recreation/

20. exp Sports/

21. Dance/

22. (exercise ${ }^{\star}$ adj2 aerobic $\left.{ }^{\star}\right) \cdot m p$.

23. sport $^{\star} . \mathrm{mp}$.

24. ((lifestyle or life style) adj5 activ*).mp.

25. (dance ${ }^{\star}$ or dancing).mp.

26.10 or 11 or 12 or 13 or 14 or 15 or 16 or 17 or 18 or 19 or 20 or 21 or 22 or 23 or 24 or 25

27. Diets/

28. exp Nutrition/ or Nutrition*.mp.

29. (health ${ }^{\star}$ adj2 eat $\left.{ }^{\star}\right) \cdot m p$.

30. Child Nutrition Sciences.mp.

31. fruit*.mp.

32. vegetable*.mp.

33. canteen ${ }^{\star} \cdot \mathrm{mp}$.

34. Food Services.mp.

35. $\mathrm{menu}^{\star} . \mathrm{mp}$.

36. (calorie or calories or kilojoule*).mp.

37. Food Intake/ or Energy Intake.mp.

38. energy density.mp.

39. Eating.mp.

Strategies to improve the implementation of healthy eating, physical activity and obesity prevention policies, practices or programmes

Copyright $\odot 2020$ The Cochrane Collaboration. Published by John Wiley \& Sons, Ltd. 
40. Eating Behavior/

41. feeding behavio? $r^{\star} . \mathrm{mp}$.

42. dietary intake.mp.

43. Food/

44. ((carbonated or sweetened or soft) adj (drink* or beverage $\left.\left.{ }^{\star}\right)\right) . \mathrm{mp}$.

45. soda.mp.

46. Dietary Fat*.mp.

47. confectionary.mp.

48. (school adj2 (lunch* or meal*)).mp.

49. feeding program ${ }^{\star} . \mathrm{mp}$.

50. food program ${ }^{\star} . \mathrm{mp}$.

51. (nutrition* adj2 program*).mp.

52. cafeteria*.mp.

53.27 or 28 or 29 or 30 or 31 or 32 or 33 or 34 or 35 or 36 or 37 or 38 or 39 or 40 or 41 or 42 or 43 or 44 or 45 or 46 or 47 or 48 or 49 or 50 or 51 or 52

\section{9 or 26 or 53}

55. preschool students/ or nursery school students/

56. $\left(\right.$ pre-school $^{\star}$ or preschool $\left.{ }^{\star}\right) \cdot \mathrm{mp}$.

57. Day Care Centers/ or Child Day Care/

58. (childcare* or child care $\left.{ }^{\star}\right) \cdot \mathrm{mp}$.

59. (daycare* or day care $\left.{ }^{\star}\right) \cdot \mathrm{mp}$.

60. early child ${ }^{\star} . \mathrm{mp}$.

61. (nursery or nurseries).mp.

62. Kindergarten Students/ or Kinder ${ }^{\star} . \mathrm{mp}$.

63.55 or 56 or 57 or 58 or 59 or 60 or 61 or 62

64. randomi?ed controlled trial*.mp.

65. Clinical Trials/

66. trial*.tw.

67. double blind.tw.

68. single blind.tw.

69. experiment ${ }^{\star}$.tw.

70. (pretest or pre test).tw.

71. (posttest or post test).tw.

72. (pre post or prepost).tw.

73. before after.tw.

74. qua?i randomi?ed.tw.

Strategies to improve the implementation of healthy eating, physical activity and obesity prevention policies, practices or programmes

Copyright $\odot 2020$ The Cochrane Collaboration. Published by John Wiley \& Sons, Ltd. 
75. stepped wedge.tw.

76. (non randomi?ed or nonrandomi?ed).tw.

77. interrupted time series.tw.

78. multiple baseline.tw.

79. regression discontinuity.tw.

80. comprehensive cohort.tw.

81. random*.ab.

82.64 or 65 or 66 or 67 or 68 or 69 or 70 or 71 or 72 or 73 or 74 or 75 or 76 or 77 or 78 or 79 or 80 or 81

83. implement*.mp.

84. dissemin*.mp.

85. adopt*.mp.

86. practice $^{\star} . \mathrm{mp}$.

87. organi?ational change ${ }^{\star} . \mathrm{mp}$.

88. diffus*.mp.

89. (system ${ }^{\star}$ adj2 change*).tw.

90. quality improvement ${ }^{\star} . \mathrm{mp}$.

91. transform ${ }^{\star} . \mathrm{mp}$.

92. translat*.mp.

93. transfer*.mp.

94. uptake*.mp.

95. sustainab*.mp.

96. institutionali*.mp.

97. routin ${ }^{\star} . \mathrm{mp}$.

98. maintenance.mp.

99. capacity.mp.

100. incorporat ${ }^{\star} . \mathrm{mp}$.

101. adher ${ }^{\star} . \mathrm{mp}$.

102. ((polic ${ }^{\star}$ or practice ${ }^{\star}$ or program ${ }^{\star}$ or innovation ${ }^{\star}$ ) adj5 (performance or feedback or prompt ${ }^{\star}$ or reminder ${ }^{\star}$ or incentive* or penalt* or communicat ${ }^{\star}$ or social market* or professional development or network $^{\star}$ or leadership or opinion leader ${ }^{\star}$ or consensus process ${ }^{\star}$ or change

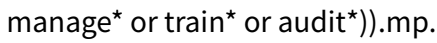

103. integrat*.mp.

104. scal* up.mp.

105. Quality Control/

106. quality of services/

107. Program Evaluation/

Strategies to improve the implementation of healthy eating, physical activity and obesity prevention policies, practices or programmes

Copyright (c) 2020 The Cochrane Collaboration. Published by John Wiley \& Sons, Ltd. 
108. 83 or 84 or 85 or 86 or 87 or 88 or 89 or 90 or 91 or 92 or 93 or 94 or 95 or 96 or 97 or 98 or 99 or 100 or 101 or 102 or 103 or 104 or 105 or 106 or 107

109. 54 and 63 and 82 and 108

\section{ERIC search strategy}

(obes* OR "weight gain" OR "weight loss" OR overweight OR "over weight" OR overeat* OR over eat* OR "weight change*" OR ((bmi OR "body mass index") AND (gain OR loss OR change)) OR Exercise* OR "physical inactivity" OR "physical activity" OR "Motor Activity" OR "physical education" OR "physical training" OR "Physical Fitness" OR sedentary OR "leisure activit*" OR sport* OR dance* OR (("life style" OR lifestyle) AND activ*) OR Diet OR nutrition* OR (health* AND eat*) OR "Child Nutrition*" OR fruit* OR vegetable* OR canteen* OR menu* OR calorie OR calories OR kilojoule* OR "Energy Intake" OR "energy density" OR Eating OR "Feeding Behavio*" OR "dietary intake" OR food OR ((carbonated OR sweetened OR soft) AND (drink* OR beverage*)) OR soda OR "Dietary Fat " OR confectionary OR (school AND (lunch* OR meal*)) OR "feeding program*" OR cafeteria*)

AND ("pre-school*" or preschool* or childcare* or "child care*" or daycare* or "day care*" or "early child" or nursery or nurseries or Kinder*)

AND (Random* or trial* or "double blind" or "single blind" or experiment* or pretest or "pre test" or posttest or "post test" or "pre post" or prepost or "before after" or "stepped wedge" or nonrandomi?ed or "interrupted time series" or "multiple baseline" or "regression discontinuity" or "comprehensive cohort" or "cohort stud"” OR "cohort analysis")

AND ("quality control" OR "health promotion" OR "quality assessment" OR "outcome assessment" OR "process assessment" OR "program evaluation" OR "total quality management" OR "health care quality" OR Implement* or dissemin* or adopt* or practice* or "organi?ational

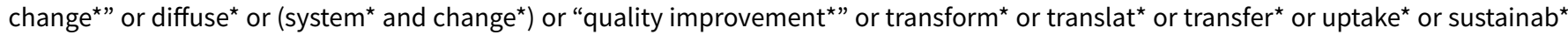
or institutionali* or routin ${ }^{\star}$ or maintenance or capacity or incorporate* or adher ${ }^{\star}$ or ((polic ${ }^{\star}$ or practice* or program ${ }^{\star}$ or innovation $\left.{ }^{\star}\right)$ and (performance or feedback or prompt ${ }^{\star}$ or reminder ${ }^{\star}$ or incentive* or penalt ${ }^{\star}$ or communicat* or social market ${ }^{\star}$ or professional development or network ${ }^{\star}$ or leadership or opinion leader or consensus process $^{\star}$ or change manage or train $^{\star}$ or audit $\left.^{\star}\right)$ ) or integrat ${ }^{\star}$ or "scal $^{\star}$ up")

\section{CINAHL search strategy}

S1. (MH "Obesity+")

S2. (MH "Weight Gain")

S3. (MH "Weight Loss+")

S4. "weight gain" or "weight loss"

S5. overweight or "over weight" or overeat* or "over eat*"

S6. "weight change*"

S7. ((bmi or "body mass index") n2 (gain or loss or change))

S8. obes*

\section{S9. S1 OR S2 OR S3 OR S4 OR S5 OR S6 OR S7 OR S8}

\section{S10. (MH "Exercise+")}

S11. "physical inactivity"

S12. (MH "Physical Activity") OR "physical activity"

S13. (MH "Motor Activity+")

S14. "physical education" or "physical training"

S15. (MH "Physical Education and Training+")

S16. (MH "Physical Fitness")

S17. "sedentary"

S18. (MH "Life Style+")

S19. (MH "Leisure Activities+")

Strategies to improve the implementation of healthy eating, physical activity and obesity prevention policies, practices or programmes within childcare services (Review)

Copyright (c) 2020 The Cochrane Collaboration. Published by John Wiley \& Sons, Ltd. 
S20. (MH "Sports+")

S21. (MH "Dancing+")

S22. exercis* $n 2$ aerobic*

S23. sport*

S24. ("life style" or lifestyle) n5 activ*

S25. dance* or dancing

S26. S10 OR S11 OR S12 OR S13 OR S14 OR S15 OR S16 OR S17 OR S18 OR S19 OR S20 OR S21 OR S22 OR S23 OR S24 OR S25

S27. (MH "Diet+")

S28. "nutrition*"

S29. (MH "Nutrition")

S30. health* n2 eat*

S31. (MH "Child Nutrition")

S32. (MH "Fruit+")

S33. (MH "Vegetables") OR "vegetable*"

S34. fruit*

S35. "canteen*"

S36. (MH "Food Services")

S37. (MH "Menu Planning") OR "menu*"

S38. calorie or calories or kilojoule*

S39. (MH "Energy Intake") OR (MH "Food Intake")

S40. (MH "Energy Density") OR "Energy Density"

S41. "feeding behavio? $r^{\star} "$

S42. (MH "Eating") OR (MH "Eating Behavior")

S43. "dietary intake"

S44. (MH "Food Habits")

S45. (MH "Food")

S46. (MH "Carbonated Beverages") OR "soft drink*"

S47. soda

S48. "sweetened drink*"

S49. (MH "Dietary Fats")

S50. "confectionary" OR (MH "Candy")

S51. school n2 (lunch* or meal ${ }^{\star}$ )

S52. "feeding program*"

S53. "food program*"

S54. (nutrition* $\mathrm{n} 2$ program)

Strategies to improve the implementation of healthy eating, physical activity and obesity prevention policies, practices or programmes 
S55. cafeteria*

S56. (MH "Nutritional Status")

S57. S27 OR S28 OR S29 OR S30 OR S31 OR S32 OR S33 OR S34 OR S 35 OR S36 OR S37 OR S38 OR S 39 OR S40 OR S41 OR S42 OR S43 OR S44 OR S45 OR S46 OR S47 OR S48 OR S49 OR S50 OR S51 OR S52 OR S53 OR S54 OR S55 OR S56

\section{S58. S9 OR S26 OR S57}

S59. (MH "Child, Preschool")

S60. "pre-schook*" or preschool*

S61. (MH "Child Day Care") OR (MH "Child Care Providers") OR (MH "Child Care (Saba CCC)") OR (MH "Child Care")

S62. childcare* or "child care*"

S63. daycare* or "day care*"

S64. "early child*"

S65. (MH "Schools, Nursery")

S66. nursery or nurseries

S67. Kinder*

S68. S59 OR S60 OR S61 OR S62 OR S63 OR S64 OR S65 OR S66 OR S67

S69. (MH "Randomized Controlled Trials")

S70. (MH "Clinical Trials")

S71. TI trial* OR AB trial*

S72. (MH "Double-Blind Studies") OR "double blind"

S73. (MH "Single-Blind Studies") OR "single blind"

S74. (MH "Experimental Studies") OR "experiment*"

S75. TI ( pretest or "pre test" ) OR AB (pretest or "pre test" )

S76. TI ( posttest or "post test" ) OR AB ( posttest or "post test" )

S77. TI ( "pre post" or prepost ) OR AB ( "pre post" or prepost)

S78. TI "before after" OR AB "before after"

S79. TI "qua?i randomi?ed" OR AB "qua?i randomi?ed"

S80. TI "stepped wedge" OR AB "stepped wedge"

S81. TI ( "non randomi?ed" or nonrandomi?ed ) OR AB ( "non randomi?ed" or nonrandomi?ed )

S82. TI "interrupted time series" OR AB "interrupted time series"

S83. TI "multiple baseline" OR AB "multiple baseline"

S84. TI "regression discontinuity" OR AB "regression discontinuity"

S85. TI "comprehensive cohort" OR AB "comprehensive cohort"

S86. $A B$ random*

S87. S69 OR S70 OR S71 OR S72 OR S73 OR S74 OR S75 OR S76 OR S77 OR S78 OR S79 OR S80 OR S81 OR S82 OR S83 OR S84 OR S85 OR S86 S88. implement ${ }^{\star}$ 
S89. dissemin*

S90. adopt ${ }^{\star}$

S91. practice*

S92. "organi?ational change*"

S93. diffus*

S94. system ${ }^{\star} 2$ change*

S95. "quality improvement*"

S96. transform*

S97. translat*

S98. transfer ${ }^{\star}$

S99. uptake*

S100. sustainab*

S101. institutionali*

S102. routin*

S103. maintenance

S104. capacity

S105. adher*

S106. ((polic* or practice ${ }^{\star}$ or program ${ }^{\star}$ or innovation ${ }^{\star}$ ) n5 (performance or feedback or prompt* or reminder ${ }^{\star}$ or incentive ${ }^{\star}$ or penalt ${ }^{\star}$ or communicat $^{\star}$ or social market* or professional development or network ${ }^{\star}$ or leadership or opinion leader ${ }^{\star}$ or consensus process ${ }^{\star}$ or change \left.${\text { manage* } \text { or train }^{\star} \text { or audit }}^{\star}\right)$ )

S107. integrat*

S108. scal* up

S109. incorporat*

S110. (MH "Health Promotion")

S111. (MH "Quality Assessment")

S112. (MH "Process Assessment (Health Care)")

S113. (MH "Program Evaluation")

S114. S88 OR S89 OR S90 OR S91 OR S92 OR S93 OR S94 OR S95 OR S96 OR S97 OR S98 OR S99 OR S100 OR S101 OR S102 OR S103 OR S104 OR S105 OR S106 OR S107 OR S108 OR S109 OR S110 OR S111 OR S112 OR S113

S115 S58 AND S68 AND S87 AND S114 Limited to June 21016+

\section{SCOPUS search strategy}

TITLE-ABS-KEY ( ( obes* OR "weight gain" OR "weight loss" OR overweight OR "over weight" OR overeat* OR over AND eat* OR "weight change*" OR ( ( bmi OR "body mass index" ) AND ( gain OR loss OR change ) ) OR exercise* OR "physical inactivity" OR "physical activity" OR "Motor Activity" OR "physical education" OR "physical training" OR "Physical Fitness" OR sedentary OR "leisure activit*" OR sport* OR dance* OR ( ( "life style" OR lifestyle ) AND activ*) OR diet OR nutrition* OR ( health* AND eat* ) OR "Child Nutrition*" OR fruit* OR vegetable* OR canteen* OR menu* OR calorie OR calories OR kilojoule* OR "Energy Intake" OR "energy density" OR eating OR "Feeding Behavio*" OR "dietary intake" OR food OR ( ( carbonated OR sweetened OR soft ) AND ( drink* OR beverage*)) OR soda OR "Dietary Fat ${ }^{\star} "$ OR confectionary OR ( school AND ( lunch* OR meal*)) OR "feeding program*" OR cafeteria* ) ) AND TITLE-ABS-KEY ( ( pre-school*" OR preschool* OR childcare* OR "child care*" OR daycare* OR "day care*" OR "early child*" OR nursery OR nurseries OR kinder* ) ) AND TITLE-ABS-KEY ( ( random* OR trial* OR "double blind" OR "single blind" OR experiment* OR pretest OR "pre test" OR posttest

Strategies to improve the implementation of healthy eating, physical activity and obesity prevention policies, practices or programmes 120 within childcare services (Review)

Copyright (c) 2020 The Cochrane Collaboration. Published by John Wiley \& Sons, Ltd. 
OR "post test" OR "pre post" OR prepost OR "before after" OR "stepped wedge" OR nonrandomi?ed OR "interrupted time series" OR "multiple baseline" OR "regression discontinuity" OR "comprehensive cohort" OR "cohort stud*" OR "cohort analysis" ) ) AND TITLE-ABSKEY ( ( "quality control" OR "health promotion" OR "quality assessment" OR "outcome assessment" OR "process assessment" OR "program evaluation" OR "total quality management" OR "health care quality" OR implement* OR dissemin* OR adopt* OR practice* OR "organi? ational change $e^{\star}$ OR diffuse* OR ( system* AND change* ) OR "quality improvement ${ }^{\star}$ " OR transform* OR translat* OR transfer* OR uptake* OR sustainab* OR institutionali* OR routin* OR maintenance OR capacity OR incorporate* OR adher* OR ( ( polic* OR practice* OR program ${ }^{\star}$ OR innovation*) AND ( performance OR feedback OR prompt* OR reminder ${ }^{\star}$ OR incentive* OR penalt* OR communicat* OR social AND market* OR professional AND development OR network* OR leadership OR opinion AND leader* OR consensus AND process ${ }^{\star}$ OR change AND manage* OR train* OR audit* ) ) OR integrat* OR "scal* up" ) )

\section{Appendix 2. Additional Risk of Bias for secondary outcomes}

Alkon 2014

\begin{tabular}{|c|c|c|}
\hline Domain & Risk of bias & Support for judgement \\
\hline $\begin{array}{l}\text { Random sequence } \\
\text { generation }\end{array}$ & Unclear risk & $\begin{array}{l}\text { Authors indicate that the centers were randomly assigned to treatment } \\
\text { groups, but sequence generation procedure was not decribed. }\end{array}$ \\
\hline (selection bias) & & $\begin{array}{l}\text { One control group centre that was not able to adquately complete baseline } \\
\text { data collection was replaced by a matched centre (unclear if this was random- } \\
\text { ly chosen). }\end{array}$ \\
\hline
\end{tabular}

\begin{tabular}{l}
\hline $\begin{array}{l}\text { Allocation conceal- } \quad \text { Unclear risk } \\
\text { ment }\end{array}$ \\
(selection bias)
\end{tabular}

\begin{tabular}{lll}
\hline $\begin{array}{l}\text { Blinding of partici- } \\
\text { pants and personnel } \\
\text { (performance bias) }\end{array}$ & High risk & $\begin{array}{l}\text { Physical activity and weight: Assume through nature of the intervention that } \\
\text { centre staff and study personnel delivering the intervention not blind to the } \\
\text { study allocation and therefore potential high risk of performance bias }\end{array}$ \\
\hline $\begin{array}{l}\text { Blinding of outcome } \\
\text { assessment } \\
\text { (detection bias) }\end{array}$ & Low risk & $\begin{array}{l}\text { Physical activity and weight: Outcome assessment undertaken by blinded re- } \\
\text { search personnel and therefore risk of detection bias considered to be low. }\end{array}$ \\
\hline $\begin{array}{l}\text { Incomplete outcome } \\
\text { data }\end{array}$ & Low risk \\
$\begin{array}{l}\text { (attrition bias) } \\
\text { Selective outcome re- } \\
\text { porting }\end{array}$ & Unclear risk \\
$\begin{array}{l}\text { (reporting bias) } \\
\text { Other bias }\end{array}$ & $\begin{array}{l}\text { Complete data collected for all centres (8 control and } 9 \text { intervention), with no } \\
\text { centres excluded from the analysis - therefore risk of attrition bias considered } \\
\text { to be low }\end{array}$ \\
\hline $\begin{array}{l}\text { Unaware if any other planned outcomes were not reported - for instance, no } \\
\text { protocol found }\end{array}$ \\
\hline
\end{tabular}

\begin{tabular}{lll}
\hline Domain & Risk of bias & Support for judgement \\
\hline
\end{tabular}


(Continued)

$\begin{array}{ll}\begin{array}{l}\text { Random sequence } \\ \text { generation }\end{array} & \text { Low risk } \\ \text { random number sequence }\end{array}$

(selection bias)

$\begin{array}{ll}\begin{array}{l}\text { Allocation conceal- } \\ \text { ment }\end{array} & \begin{array}{l}\text { Statistician not involved in the project allocated the services to groups using a } \\ \text { computerised program }\end{array} \\ \text { (selection bias) } & \end{array}$

\begin{tabular}{|c|c|c|}
\hline $\begin{array}{l}\text { Blinding of partici- } \\
\text { pants and personnel } \\
\text { (performance bias) }\end{array}$ & High risk & $\begin{array}{l}\text { Assume through nature of the intervention that centre staff and study person- } \\
\text { nel delivering the intervention not blind to the study allocation and therefore } \\
\text { potential high risk of performance bias }\end{array}$ \\
\hline \multirow{2}{*}{$\begin{array}{l}\text { Blinding of outcome } \\
\text { assessment } \\
\text { (detection bias) }\end{array}$} & Low risk & $\begin{array}{l}\text { Child physical activity: Measured using pedometers with research staff blind to } \\
\text { group allocation }\end{array}$ \\
\hline & High risk & Adverse effects: service manager self-report via interview \\
\hline $\begin{array}{l}\text { Incomplete outcome } \\
\text { data } \\
\text { (attrition bias) }\end{array}$ & Low risk & $\begin{array}{l}\text { Child physical activity: Although there was } 48 \% \text { and } 44 \% \text { loss to follow-up in } \\
\text { intervention and control groups respectively, sensititvity analysis imputing } \\
\text { missing data showed no difference in outcome analysis. }\end{array}$ \\
\hline Recruitment bias & Low risk & $\begin{array}{l}\text { For the physical activity measure, children were recruited by supervisors at the } \\
\text { centre selecting a day of the week for measurement to occur. Allocation was } \\
\text { not revealed to services until after baseline data collection. }\end{array}$ \\
\hline
\end{tabular}

\begin{tabular}{|c|c|c|}
\hline Domain & Risk of bias & Support for judgement \\
\hline $\begin{array}{l}\text { Random sequence } \\
\text { generation } \\
\text { (selection bias) }\end{array}$ & Low risk & A random number function was used to generate the random sequence. \\
\hline \multirow[t]{2}{*}{$\begin{array}{l}\text { Blinding of partici- } \\
\text { pants and personnel } \\
\text { (performance bias) }\end{array}$} & Unclear risk & $\begin{array}{l}\text { Child dietary intake and physical activity levels: Services were not blind to } \\
\text { study allocation however observers were blind to allocation at the level of the } \\
\text { child and so the impact of performance bias is unclear. }\end{array}$ \\
\hline & High risk & $\begin{array}{l}\text { Acceptability and adverse effects: Services were not blind to study allocation } \\
\text { and therefore high risk of performance bias }\end{array}$ \\
\hline
\end{tabular}


(Continued)

$\begin{array}{ll}\text { Blinding of outcome } \quad \text { Low risk } & \text { Child dietary intake and physical activity levels: Observers were blind to } \\ \text { assessment } & \text { service group allocation. }\end{array}$

(detection bias)

High risk

\section{Acceptability and adverse effects:}

Services were not blind to study allocation and therefore high risk to detection bias

\begin{tabular}{l}
\hline $\begin{array}{l}\text { Incomplete outcome Low risk } \\
\text { data }\end{array}$ \\
(attrition bias) \\
\hline $\begin{array}{l}\text { Selective outcome re- Unclear risk } \\
\text { porting }\end{array}$
\end{tabular}

(reporting bias)

120/128 services (95\%) provided follow-up data.

\begin{tabular}{lll}
\hline Recruitment bias & Low risk & $\begin{array}{l}\text { The children were randomly selected by asking the room leader at each ser- } \\
\text { vice to identify the three children with the most recent birthdays. }\end{array}$ \\
\hline Baseline imbalance & Low risk & $\begin{array}{l}\text { No baseline imbalances in service characteristics. No baseline measures of } \\
\text { secondary outcomes taken }\end{array}$ \\
\hline Loss of clusters & Unclear risk & $\begin{array}{l}\text { Only follow-up data collected from random sample of services that had re- } \\
\text { mained in trial at follow-up }\end{array}$ \\
\hline $\begin{array}{l}\text { Incorrect analysis } \\
\begin{array}{l}\text { Compatibility with in- } \\
\text { dividually randomised } \\
\text { RCTs (cluster-RCTs): }\end{array}\end{array}$ & Unclear risk & The analysis appeared appropriate. Clustering effects adjusted for \\
\hline
\end{tabular}

\begin{tabular}{lll}
\hline Domain & Risk of bias & Support for judgement \\
\hline $\begin{array}{l}\text { Random sequence genera- } \\
\text { tion }\end{array}$ & Unclear risk & The random sequence generation procedure was not described. \\
(selection bias) & \\
\hline $\begin{array}{l}\text { Allocation concealment } \\
\text { (selection bias) }\end{array}$ & Unclear risk & $\begin{array}{l}\text { There was no information provided about allocation concealment and } \\
\text { therefore it was unclear if allocation was concealed. }\end{array}$ \\
\hline $\begin{array}{l}\text { Blinding of participants } \\
\text { and personnel }\end{array}$ & Unclear risk \\
(performance bias) & $\begin{array}{l}\text { Child and teacher physical activity (accelerometer-measured): There was } \\
\text { no mention that the teachers and children were blinded. However, physi- } \\
\text { cal activity was objectively measured and so it was unclear if there was a } \\
\text { risk of performance bias. }\end{array}$ \\
\hline $\begin{array}{l}\text { Blinding of outcome as- } \\
\text { sessment }\end{array}$ & Unclear risk & $\begin{array}{l}\text { Child and teacher physical activity (accelerometer-measured): Child and } \\
\text { teacher physical activity was measured using accelerometers, however, } \\
\text { outcome assessors were not blinded to allocation and so unclear risk of } \\
\text { detection bias. }\end{array}$ \\
\hline
\end{tabular}

Strategies to improve the implementation of healthy eating, physical activity and obesity prevention policies, practices or programmes 
(Continued)

\begin{tabular}{|c|c|c|}
\hline $\begin{array}{l}\text { Incomplete outcome data } \\
\text { (attrition bias) }\end{array}$ & Low risk & $\begin{array}{l}\text { All } 13 \text { centres provided post-intervention data. Seven children ( } 4 \text { interven- } \\
\text { tion, } 3 \text { control) of the } 182 \text { children did not provide post-intervention data } \\
\text { ( } 4 \% \text { attrition). }\end{array}$ \\
\hline $\begin{array}{l}\text { Selective outcome report- } \\
\text { ing } \\
\text { (reporting bias) }\end{array}$ & Low risk & $\begin{array}{l}\text { The outcomes reported aligned with those outlined in the trial registra- } \\
\text { tion. }\end{array}$ \\
\hline Recruitment bias & Low risk & All children within participating centres/classes invited to participate \\
\hline Baseline imbalance & Low risk & No baseline imbalances in service characteristics or outcomes \\
\hline Loss of clusters & Low risk & No loss of sites \\
\hline $\begin{array}{l}\text { Compatibility with indi- } \\
\text { vidually randomised RCTs } \\
\text { (cluster-RCTs): }\end{array}$ & Unclear risk & No evidence to make assessment \\
\hline
\end{tabular}

\begin{tabular}{|c|c|c|}
\hline Domain & Risk of bias & Support for judgement \\
\hline $\begin{array}{l}\text { Random sequence } \\
\text { generation } \\
\text { (selection bias) }\end{array}$ & Low risk & $\begin{array}{l}\text { A random number function in Microsoft Excel was used to generate the ran- } \\
\text { dom sequence. }\end{array}$ \\
\hline $\begin{array}{l}\text { Allocation conceal- } \\
\text { ment } \\
\text { (selection bias) }\end{array}$ & Low risk & $\begin{array}{l}\text { Consenting childcare services were immediately randomly allocated using a } \\
\text { random number function in the statistical software package SAS. }\end{array}$ \\
\hline $\begin{array}{l}\text { Blinding of partici- } \\
\text { pants and personnel }\end{array}$ & Unclear risk & $\begin{array}{l}\text { Child food group consumption (service level): Childcare services were aware } \\
\text { of their group allocation, however, it was unclear how this impacted on the } \\
\text { risk of performance bias for this outcome. }\end{array}$ \\
\hline
\end{tabular}

(performance bias)

High risk

Unclear risk
Adverse effects reported by service cooks: Childcare service staff were aware of their group allocation.

Adverse effects reported by children/parents: It was unclear if adverse effects reported by children/parents were influenced by performance bias.

\begin{tabular}{lll}
\hline $\begin{array}{l}\text { Blinding of outcome } \\
\text { assessment }\end{array}$ & Low risk & $\begin{array}{l}\text { Child food group consumption (service level): All trial outcome data collec- } \\
\text { tors were blinded. }\end{array}$ \\
\cline { 2 - 3 } (detection bias) & High risk & $\begin{array}{l}\text { Adverse effects reported by service cooks: Childcare service staff were } \\
\text { aware of their group allocation and therefore there was a high risk of detectio } \\
\text { bias. }\end{array}$ \\
\hline Unclear risk & $\begin{array}{l}\text { Adverse effects reported by children/parents: It was unclear if adverse ef- } \\
\text { fects reported by children/parents were influenced by detection bias. }\end{array}$
\end{tabular}


(Continued)

$\begin{array}{ll}\begin{array}{l}\text { Incomplete outcome } \\ \text { data }\end{array} & \text { At follow-up, 24/26 (92\%) in the intervention and 20/28 (71\%) in the control } \\ \text { provided their menu. Missing data was imputed. }\end{array}$

(attrition bias)

$\begin{array}{ll}\begin{array}{l}\text { Selective outcome re- } \\ \text { porting }\end{array} & \text { High risk } \\ \begin{array}{l}\text { (reporting bias) } \\ \text { specified in the protocol but not reported in the outcome paper and the ser- } \\ \text { vice-level child food group servings consumption was reported using a differ- } \\ \text { ent measure then was specified in the protocol. }\end{array}\end{array}$

\section{Other bias}

\begin{tabular}{lll}
\hline Domain & Risk of bias & Support for judgement \\
\hline $\begin{array}{ll}\text { Random sequence } \\
\text { generation }\end{array}$ & High risk & $\begin{array}{l}\text { Nonrandomised trial: nonrandom allocation (no random sequence generat- } \\
\text { ed). Therefore. high risk of selection bias }\end{array}$ \\
(selection bias) & &
\end{tabular}

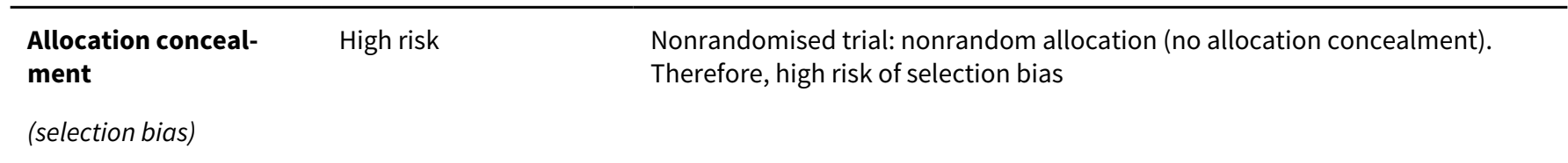

\begin{tabular}{lll}
\hline $\begin{array}{l}\text { Blinding of partici- } \\
\text { pants and personnel } \\
\text { (performance bias) }\end{array}$ & High risk & $\begin{array}{l}\text { Obesity (BMI): There was no mention that the participants and personnel } \\
\text { were blinded and, therefore, there was a high risk of performance bias. }\end{array}$ \\
\cline { 2 - 3 } & High risk & $\begin{array}{l}\text { Child diet: There was no mention that the participants and personnel were } \\
\text { blinded and, therefore, there was a high risk of performance bias. }\end{array}$ \\
\cline { 2 - 3 } & High risk & $\begin{array}{l}\text { Child physical activity: There was no mention that the participants and per- } \\
\text { sonnel were blinded and, therefore, there was a high risk of performance bias. }\end{array}$ \\
\hline $\begin{array}{l}\text { Blinding of outcome } \\
\text { assessment } \\
\text { (detection bias) }\end{array}$ & Low risk & $\begin{array}{l}\text { Obesity (BMI): Child height and weight were measured using stadiometers } \\
\text { and digital scales. }\end{array}$ \\
\cline { 2 - 3 } & High risk & $\begin{array}{l}\text { Child diet (parent surveys): There was no mention that the participants were } \\
\text { blinded and, therefore, there was a high risk of detection bias. }\end{array}$ \\
\cline { 2 - 3 } & High risk & $\begin{array}{l}\text { Physical activity (parent surveys): There was no mention that the partici- } \\
\text { pants were blinded and, therefore, there was a high risk of detection bias. }\end{array}$ \\
\hline $\begin{array}{l}\text { Incomplete outcome } \\
\text { data }\end{array}$ & Low risk & No sites dropped out. \\
(attrition bias) & & \\
\hline
\end{tabular}

Selective outcome re- Low risk All measures aligned between the Sharma and Hoelscher papers.
porting

(reporting bias) 
(Continued)

$\begin{array}{ll}\text { Potential confounding Low risk } & \text { Various known confounders that were considered for inclusion into each of } \\ & \text { the regression models included: city (Houston and Austin), child race/ethnicity } \\ \text { and gender, parental race, and education level. }\end{array}$

\begin{tabular}{|c|c|c|}
\hline Recruitment bias & Low risk & All parents/children were invited to participate. \\
\hline Baseline imbalance: & Unclear risk & $\begin{array}{l}\text { Significantly more parents in the intervention centers reported receiving SNAP } \\
\text { benefits compared with those in the comparison centers across both years } \\
\text { of measurement. For year } 1 \text {, children in the comparison centres were slightly } \\
\text { younger than those in the intervention group. }\end{array}$ \\
\hline
\end{tabular}

\begin{tabular}{lll}
\hline Loss of clusters: & Low risk & No loss of clusters \\
\hline Incorrect analysis: & Low risk & Adjustment for potential clustering in analysis \\
\hline $\begin{array}{l}\text { Compatibility with in- } \\
\text { dividually randomised } \\
\text { RCTs (cluster-RCTs): }\end{array}$ & Unclear risk & No evidence to make assessment \\
\hline
\end{tabular}

\begin{tabular}{|c|c|c|}
\hline Domain & Risk of bias & Support for judgement \\
\hline $\begin{array}{l}\text { Random sequence } \\
\text { generation } \\
\text { (selection bias) }\end{array}$ & Low risk & $\begin{array}{l}\text { A list of random, unique, unsorted numbers was generated using randomiz- } \\
\text { er.org. }\end{array}$ \\
\hline $\begin{array}{l}\text { Allocation conceal- } \\
\text { ment } \\
\text { (selection bias) }\end{array}$ & Low risk & $\begin{array}{l}\text { Eligible childcare centres were listed in alphabetical order and a list of random } \\
\text { numbers generated. }\end{array}$ \\
\hline $\begin{array}{l}\text { Blinding of partici- } \\
\text { pants and personnel } \\
\text { (performance bias) }\end{array}$ & Unclear risk & $\begin{array}{l}\text { Child BMI: The health care workers and childcare providers were not blinded to } \\
\text { treatment allocation and there was no mention if children were blinded. How- } \\
\text { ever, the impact this would have on child BMI was unclear. }\end{array}$ \\
\hline $\begin{array}{l}\text { Blinding of outcome } \\
\text { assessment } \\
\text { (detection bias) }\end{array}$ & Low risk & $\begin{array}{l}\text { Child BMI: Child weight and height was measured using a digital scale and sta- } \\
\text { diometer, therefore, low risk of detection bias. }\end{array}$ \\
\hline $\begin{array}{l}\text { Incomplete outcome } \\
\text { data } \\
\text { (attrition bias) }\end{array}$ & Low risk & $\begin{array}{l}\text { At the 2-year follow-up, } 9 \text { ( } 4 \text { in intervention, } 5 \text { in comparison) of the } 43 \text { centres } \\
\text { had missing data ( } 21 \% \text { attrition). Low risk of attrition bias }\end{array}$ \\
\hline $\begin{array}{l}\text { Selective outcome re- } \\
\text { porting } \\
\text { (reporting bias) }\end{array}$ & Unclear risk & $\begin{array}{l}\text { There was no study protocol, therefore, it was unclear if there was selective } \\
\text { outcome reporting. }\end{array}$ \\
\hline Recruitment bias: & Low risk & All parents/children were invited to participate. \\
\hline
\end{tabular}


(Continued)

Baseline imbalance: Unclear risk Some baseline imbalances, but unknown whether these biased outcome. CCHP + HAP centres served significantly older children than CCHP + HAP Delayed centres in 2011-2012 and 2012-2013. The CCHP + HAP centres had a significantly smaller prevalence of overweight or obesity at autumn enrollment, compared to CCHP + HAP Delayed centers, in the baseline year (2011-2012). Intervention centres also had on average more children enrolled per centre than control centres (i.e. difference in size).

\begin{tabular}{lll}
\hline Loss of clusters: & Low risk & Low risk of loss of clusters - similar \% of centres lost across groups \\
\hline Incorrect analysis: & Low risk & $\begin{array}{l}\text { The intracluster correlation coefficient (ICC), measure of within-child care cen- } \\
\text { tre variance, relative to between-child care centre variance, was estimated to } \\
\text { describe clustering in the outcome data in the follow-up year and Implementa- } \\
\text { tion year } 2 .\end{array}$ \\
\hline $\begin{array}{l}\text { Compatibility with in- } \\
\text { dividually randomised } \\
\text { RCTs (cluster-RCTs): }\end{array}$ & Unclear risk & No evidence to make assessment \\
\hline
\end{tabular}

\begin{tabular}{|c|c|c|}
\hline Domain & Risk of bias & Support for judgement \\
\hline $\begin{array}{l}\text { Random sequence } \\
\text { generation } \\
\text { (selection bias) }\end{array}$ & High risk & $\begin{array}{l}\text { No random allocation to control and intervention conditions (random alloca- } \\
\text { tion to } 1 \text { or } 2 \text { intervention conditions) }\end{array}$ \\
\hline $\begin{array}{l}\text { Allocation conceal- } \\
\text { ment } \\
\text { (selection bias) }\end{array}$ & High risk & As above \\
\hline $\begin{array}{l}\text { Blinding of partici- } \\
\text { pants and personnel } \\
\text { (performance bias) }\end{array}$ & High risk & $\begin{array}{l}\text { Child diet and weight status: Due to nature of the intervention, centres and } \\
\text { study personnel delivering the intervention would not have been blind to the } \\
\text { study allocation and therefore potential high risk of performance bias }\end{array}$ \\
\hline $\begin{array}{l}\text { Blinding of outcome } \\
\text { assessment } \\
\text { (detection bias) }\end{array}$ & Unclear risk & $\begin{array}{l}\text { Child diet and weight status: No information provided on whether research } \\
\text { personnel undertaking menu asessment and other data collection were blind } \\
\text { to group allocation }\end{array}$ \\
\hline $\begin{array}{l}\text { Incomplete outcome } \\
\text { data } \\
\text { (attrition bias) }\end{array}$ & Low risk & $\begin{array}{l}\text { Child diet and weight status: Practice level outcome of the review - menu data } \\
\text { collected on all intervention }(n=6) \text { and control centres }(n=3) \text { pre and post-in- } \\
\text { tervention. No information provided on difference in groups in regards to char- } \\
\text { acteristics of parents/children lost to follow-up }\end{array}$ \\
\hline $\begin{array}{l}\text { Selective outcome re- } \\
\text { porting } \\
\text { (reporting bias) }\end{array}$ & Low risk & $\begin{array}{l}\text { Methodology paper also listed physiologic measures - appeared that these } \\
\text { were published elsewhere }\end{array}$ \\
\hline
\end{tabular}




\author{
Recruitment bias Unclear risk
}

Unclear how parents/children from centres selected - whether all invited or selection process undertaken and, therefore, whether there was bias in selection of participants to clusters
Potential confounding Unclear risk

Nonrandomised design - adjustment for confounding factors: no information provided

\section{Appendix 3. Prespecified changes for Review Update - editorial approval received August 2018}

\begin{tabular}{|c|c|}
\hline Protocol section & Appraisal points \\
\hline $\begin{array}{l}\text { Background and re- } \\
\text { search question }\end{array}$ & $\begin{array}{l}\text { Review and update } \\
\text { background section, in- } \\
\text { cluding supporting ref- } \\
\text { erences to take account } \\
\text { of any changes that } \\
\text { may have occurred. } \\
\text { This should include up- } \\
\text { dating any new infor- } \\
\text { mation and current pol- } \\
\text { icy debates on the top- } \\
\text { ic. }\end{array}$ \\
\hline
\end{tabular}

Inclusion criteria

- Review current PICO(s) and amend in light of new knowledge - Identify any changes in usual care standards - Check for standardised core outcome sets, such as those developed in collaboration with the core outcome measures in effectiveness trials (COMET) initiative (www.comet-initiative.org) or by guideline groups since the original review - Check for any relevant patient-reported outcomes to include subsequent to the original review

- Consider any new studies with less risk of bias that might warrant a stricter study design inclusion criteria (where the older version, when there was a dearth of evidence, included observational or quasi-randomised comparisons)

\author{
Appraisal points
}

The review update will not involve a change in research question or aim. The primary aim of the review is to examine the effectiveness of strategies to improve the implementation of healthy eating, physical activity and obesity prevention policies, practices or programmes within childcare services.

The background section of the review will be updated to include current statistics regarding the condition (obesity) and the intervention.

The PICO developed for the original review will be closely replicated for the review update. There will be no changes to the type of population group, interventions or comparison groups previously included in the original review.

However, two adjustments will be made to the secondary outcomes measured. The original review included a secondary outcome describing the impact of strategies on childcare service staff skills, knowledge and attitude. This will be removed from the review update.

The updated review will include a secondary outcome examining implementation acceptability, adoption, penetration, sustainability and appropriateness. As outlined by Proctor in the study, Outcomes for Implementation Research: Conceptual Distinctions, Measurement Challenges, and Research Agen$d a$, it is recommended that all implementation trials assess these implementation outcomes. For the review, these implementation outcomes will be operationally defined as follows:

- Acceptability: Defined as the perception among implementation stakeholders that a given treatment service, practice or innovation is agreeable, palatable or satisfactory. Measures of acceptability assessed at the individual or organisational level will be included such as surveys of staff or managers of childcare services regarding their experience of features of the intervention.

- Adoption: any measure of uptake, including intention, initial decision, or action to try and implement potentially effective interventions, programmes or services. These could include decisions by managers of childcare services to take up a potentially effective service, or individual staff interventions to deliver potentially effective services.

- Penetration: integration of a practice within a service setting or its sub-settings. Include any measure of penetration at the individual or organisational level. For example, proportion of eligible individuals (or childcare services) that receive an intervention (or implement an intervention) of the total num- 
ber eligible to do so. These could include the proportion of all childcare services eligible for an intervention that actually receive it.

- Sustainability: defined as the extent to which a newly implemented intervention, programme or service is maintained. Measures of sustainability must require successful implementation in part or in full, of an intervention, programme or service. Include any measure of ongoing sustainability of implementation of intervention elements assessed at least 6 months following a measure of successful implementation. This could include the proportion of childcare services maintaining implementation of all elements of a program 12 months following the provision of implementation support.

- Appropriateness: defined as the perceived fit, relevance of the compatibility of an innovation of evidence-based practice for a given setting, provider or consumer, and/or perceived fit of the innovation to address a particular problem. Measures of appropriateness assessed at the individual of organisational level will be included, such as surveys of staff or managers of childcare services regarding their perception of the consistency of the implementation of a new intervention with their skill set or work expectations.

The study design inclusion criteria will remain the same for the review update. Any study (randomised, including cluster-randomised, or non-randomised) with a parallel control group that compares an implementation strategy with no intervention or 'usual' practice; or two or more alternative strategies to improve implementation, will be considered and screened for eligibility.

Methods

\begin{abstract}
- Appraise and update the methods pending relevant methodological advancements or developments. For example, if there are new tools for assessing the risk of bias of individual studies or appraising the quality of a body of evidence (e.g. GRADE)

- Update or include a 'Summary of findings' table, which is recommended for all systematic reviews, because it improves the clarity, understanding, and interpretation of the findings of a systematic review, and rapidly reduces the amount of time readers require to find key information
\end{abstract}

- Any new subanalysis needed

- Any substantive change in the review structure
The updated review will include a minor refinement of the search strategy for electronic databases used in the previous review. This refinement aligns with feedback received from the Cochrane Editorial team regarding the search strategy used in another review by the same lead author.

Due to the inclusion of an additional secondary outcome in the review update, studies included in the original review will be re-examined to assess the new secondary outcome. Any data from studies in the original review that assessed the new secondary outcome will be extracted and included in the review update.

Despite the addition of a new secondary outcome, the amended search will only be applied to the review update date range ( $27^{\text {th }}$ June 2016 - present). As per the original review, the primary outcome of the review update is the implementation of policies, practices or programmes in centre-based childcare services to promote healthy eating, physical activity or obesity prevention. Therefore, any eligible studies that assess the primary outcome will be included in the review. These studies will then be further examined to determine if any secondary outcomes were also evaluated, with relevant data then extracted. As per the original review, studies that do not assess the primary outcome, despite potentially assessing secondary outcomes, will be excluded from the review update.

As per the original review, the review update will appraise the quality of evidence using the GRADE method and include a 'Summary of findings' table to increase clarity and understanding for the reader. We will, however, report a SOF table for RCTs separate from non-RCTs in line with GRADE recommendations.

The updated review will also include methodological analysis advancements utilised in the Cochrane review titled Audit and Feedback: effects on professional practice and healthcare outcomes (http://cochranelibrary-wiley.com/doi/10.1002/14651858.CD000259.pub3/full). Authors of the review update intend to formally pool the trial using an adjusted median effect and conduct analyses in STATA, as described in the Audit and Feedback review. 
WHAT'S NEW

\begin{tabular}{lll}
\hline Date & Event & Description \\
\hline 23 September 2019 & $\begin{array}{l}\text { New citation required and conclusions } \\
\text { have changed }\end{array}$ & $\begin{array}{l}\text { Following the publication of this review in 2016, the updated } \\
\text { search conducted on February 22 2019 identified an addition- } \\
\text { al 11 studies as eligible for inclusion. The addition of new stud- } \\
\text { ies strengthened the evidence of the effectiveness of strategies } \\
\text { to improve the implementation of policies, practices and pro- } \\
\text { grammes within childcare services. }\end{array}$ \\
& \\
&
\end{tabular}

\section{CONTRIBUTIONS OF AUTHORS}

Luke Wolfenden, Courtney Barnes and Jannah Jones led the development of the review. Debbie Booth developed the search strategy. All authors have contributed to the conception and conduct of the research. Jannah Jones, Courtney Barnes and Meghan Finch screened titles and abstracts and determined study eligibility. Jannah Jones, Meghan Finch, Rebecca Wyse, Alice Grady and Courtney Barnes extracted data from eligible trials. Melanie Kingsland and Flora Tzelepis assessed risk of bias. Rebecca Hodder and Courtney Barnes completed the GRADE assessment. Luke Wolfenden and Courtney Barnes led the drafting of the manuscript. All authors provided critical comment on drafts.

\section{DECLARATIONS OF INTEREST}

Luke Wolfenden, Jannah Jones, Alice Grady, Courtney Barnes and Sze Lin Yoong are currently undertaking a series of randomised trials aiming to facilitate the implementation of healthy eating and physical activity policies and practices by childcare services. Authors Luke Wolfenden, Meghan Finch, Alice Grady, Jannah Jones, Rebecca Wyse and Serene Yoong are authors on one or more trials included in the review (Finch 2012; Finch 2014; Finch 2019; Jones 2015; Seward 2017; Yoong 2016). For such trials, data extraction, 'Risk of bias' and GRADE assessments were completed by authors who were not directly involved in those studies. The authors have not received any benefit, in cash or kind, any hospitality, or any subsidy derived from any source perceived to have an interest in the outcome of the review. Melanie Kingsland, Flora Tzelepis, Meghan Finch, Rebecca Wyse, Rebecca Hodder and Debbie Booth declare no conflict of interest.

\section{SOURCES OF SUPPORT}

\section{Internal sources}

- University of Newcastle, Australia.

Salary support for review authors

- Hunter New England Population Health, Australia.

Salary support for review authors

- Hunter Medical Research Institute, Australia.

Salary support for review authors

- University of Melbourne, Australia.

Salary support for review authors

\section{External sources}

- The Australian Prevention Partnership Centre, Australia.

This research was supported by The Australian Prevention Partnership Centre through the NHMRC partnership centre grant scheme (Grant ID: GNT9100001) with the Australian Government Department of Health, NSW Ministry of Health, ACT Health, HCF and the HCF Research Foundation. 


\section{DIFFERENCES BETWEEN PROTOCOLAND REVIEW}

The following changes were made to the methods for the update of this review since the original review was conducted:

1. Following feedback provided by the Cochrane Editorial team, the search terms of the strategy for electronic databases were refined.

2. A secondary outcome examining the impact of strategies on childcare service staff knowledge, skills or attitudes has been removed from the updated review.

3. An aim to describe the acceptability, adoption, penetration, sustainability and appropriateness of such implementation strategies was added.

For each comparison, we synthesised findings by outcome, and within by measure. In the previous version, findings were synthesised for each comparison by intervention and within by outcome. Details regarding the changes made for the update of this review have been summarised in Appendix 3.

\section{N DEX TERMS}

\section{Medical Subject Headings (MeSH)}

${ }^{\star}$ Child Health Services; ${ }^{\star}$ Diet, Healthy; Exercise [ ${ }^{\star}$ physiology]; Guidelines as Topic; Health Policy; Health Promotion [ ${ }^{\star}$ methods]; Pediatric Obesity [ ${ }^{*}$ prevention \& control]; Program Development; Randomized Controlled Trials as Topic

\section{MeSH check words}

Child; Child, Preschool; Humans 